\title{
Optical Technology and Measurement for Industrial Applications Conference
}

, "Optical Technology and Measurement for Industrial Applications Conference," Proc. SPIE 11142, Optical Technology and Measurement for Industrial Applications Conference, 1114201 (21 April 2019); doi: $10.1117 / 12.2535570$ 


\section{PROCEEDINGS OF SPIE}

\section{Optical Technology and Measurement for Industrial Applications Conference}

Takeshi Hatsuzawa

Editor

22-26 April 2019

Yokohama, Japan

Published by SPIE 


\section{Contents}

vii Author Index

ix Conference Committee

\section{ORAL SESSION 1}

OPTM-1-02 Towards happy marriage between optics/photonics and Al: A mini tutorial with a historical perspective

OPTM-1-03 Optical frequency metrology with frequency combs and stabilized lasers

ORAL SESSION 2

OPTM-2-01 On Carrier Fringe Pattern Analysis

OPTM-2-02 3D profilometry by projecting polarization pattern

OPTM-2-03 Self-correction of phase errors induced by projector nonlinearity in phase-shifting fringe projection profilometry

OPTM-2-04 High-speed 3D surface measurement of rear lamp housing by automatic digital fringe projection system

OPTM-2-05 Response function measurement in photovoltaic devices with sinusoidal structured illumination

ORAL SESSION 3

OPTM-3-01 Innovations in Structured Light Methods and Optical Metrology

OMC-3-02 Development of Handy Type Full-color and Real-time 3D Measurement System Using Linear LED Device

OMC-3-03 Accuracy estimation of a 3D reconstruction method for scanning electron microscope images

OMC-3-04 Design of FPGA-based signal-processing system based on the direct phase determination method for heterodyne interferometry 


\section{ORAL SESSION 4}

OPTM-4-01 High-speed ghost imaging by deep learning

OMC-4-02 Automatic rice seed imaging system for varieties classification

OMC-4-03 Light-source color correlation of wide-field spectroscopic imaging for the adaption to spatial and temporal variations when using an unmanned aerial vehicle

\section{ORAL SESSION 5}

OPTM-5-01 Systematic error correction for phase detection in sinusoidal frequency modulation displacement measuring interferometer

OPTM-5-02 An optical angle sensor based on second harmonic generation of a mode-locked laser

OPTM-5-03 Machine learning for rapid adaptation to individual optical differences for noninvasive blood glucose sensor using mid-infrared

OPTM-5-04 Rigorous analysis of reflection spectrum of absorbing film

OPTM-5-05 Optical Design of Transmission Raman Spectrometer Based on the Plane Reflective Grating

\section{ORAL SESSION 6}

OPTM-6-01 Polarization camera performance optimization

OPTM-6-02 Snapshot imaging polarimetry based on structured interference fringes

OPTM-6-03 Spectroscopic Ellipsometry Study on Aluminium-Doped Zinc Oxide Thin Films Prepared via DC Magnetron Sputtering and HiPIMS

OPTM-6-04 Imaging ellipsometry of porous silicon

OPTM-6-05 Effect of oxide layer thickness on polarization mitigation

\section{ORAL SESSION 7}

OPTM-7-02 High-Precise Optical Shape Measurement with Full-Field Heterodyne Interferometry

OPTM-7-03 Optical profilometry of cylindrical openings for translucent objects Weak value amplification of skew aberration

OPTM-7-04 Role of the zeroth-order diffraction beam and scattering light in three-dimensional shape measurement of fine structure by detecting phase distribution based on speckle interferometry 


\section{ORAL SESSION 8}

OPTM-8-01 Numerical Analysis of Near-Field Light Intensity of Whispering Gallery Mode on Microsphere Surface with SNOM Probe

OPTM-8-02 Light attenuation in the bistatic scattering measurement in the atmosphere

OPTM-8-03 Flyable Mirrors: Laser Scanning Vibrometry Method for Monitoring Large Engineering Structures Using Drones

OPTM-8-04 Fabrication of three dimensional nano-periodic structure by the Talbot lithography using multiple exposure

OPTM-8-05 Optical Trapping of Airborne Droplet for Laser Fabrication of 3-Dimensional Structure based on Optical Trapping Potential using Radially Polarized Beam

OPTM-8-06 Evaluation of influences of thin lubricant on fringe projection measurements

POSTER SESSION

OPTM-P-01 2D and 3D Vision Based Face Recognition System

OPTM-P-02 A multi-axis space coordinate system calibration method for composite line laser measuring systems using non-feature planes and multi-angle spheres

OPTM-P-03 Tuning focal length of vari-focal lens for color 3D object reconstruction

OPTM-P-04 Active optical systems with novel metal brightness amplifiers

OPTM-P-05 Phase analysis of light carrying optical vortex for refractive index sensing

OPTM-P-06 SMS and FBG interrogation for measurement of temperature and strain using OTDR Laguerre-Gaussian self-trapped beams in optical lattices

OPTM-P-07 Development of an Anamorphic Liquid-pressure Varifocal Lens

OPTM-P-08 Digital holographic analyzer of optical fiber inhomogeneity at the soldering region

OPTM-P-09 Design of high-FOV automatic optical inspection lens for linear sensor with different magnification

OPTM-P-10 Characterization of Erbium Doped Phosphate Glasses by Terahertz Time Domain Spectroscopy

OPTM-P-11 Performance Analysis of Structured Light Elements with Various Diffraction Patterns

OPTM-P-12 Research on measurement method of coincidence degree for remote micro-objects based on parallel light 
OPTM-P-13 Detection of optical vortices using various interferometers

OPTM-P-14 Modeling of optical frequency domain reflectometer based on self-sweeping fiber laser

Proc. of SPIE Vol. $111421114201-5$

Downloaded From: https://www.spiedigitallibrary.org/conference-proceedings-of-spie on 26 Apr 2023 Terms of Use: https://www.spiedigitallibrary.org/terms-of-use 
Proc. of SPIE Vol. 11142 1114201-6

Downloaded From: https://www.spiedigitallibrary.org/conference-proceedings-of-spie on 26 Apr 2023 Terms of Use: https://www.spiedigitallibrary.org/terms-of-use 


\section{Authors}

Numbers in the index correspond to the numbers in the table of contents.

Adachi, Satoru, OPTM-5-03

Aketagawa, Masato, OPTM-3-04, OPTM-5-01

Akiyama, Taiki, OPTM-6-04

Aoki, Sadao, OPTM-5-04

Arai, Yasuhiko, OPTM-7-04

Atakaramians, Shaghik, OPTM-P-10

Banerjee, Suchandra, OPTM-6-05

Banerjee, Saswatee, OPTM-5-04

Bierig, Andreas, OPTM-8-03

Buranasiri, Prathan, OPTM-6-03

Cai, Qisheng, OPTM-P-12

Chaitavon, Kosom, OPTM-4-02

Chen, Fong-Zhi, OPTM-P-09

Cheng, Pi-Ying, OPTM-P-03

Cheng, Yuan-Chieh, OPTM-P-09

Chipman, Russell, OPTM-6-05

Chou, Chun-Han, OPTM-P-11

CHU, Bohuai, OPTM-8-01

Chu, Yushi, OPTM-P-10

Dembele, Vamara, OPTM-6-02

Deng, Qiwen, OPTM-2-05

Dey, Koustav, OPTM-P-06

Fan, Desheng, OPTM-P-10

Fu, Xinghu, OPTM-P-10

Fujigaki, Motoharu, OPTM-3-02

Gao, Huiliang, OPTM-8-02

Gao, Wei, OPTM-5-02

Gavril'eva, K., OPTM-P-13

Gelloz, Bernard, OPTM-6-04, OPTM-7-03

Guo, Hongwei, OPTM-2-03

Hagen, Nathan, OPTM-2-02, OPTM-6-01, OPTM-6-05

Han, Wei, OPTM-P-12

Hassan, Saher, OPTM-8-03

Hausotte, Tino, OPTM-8-06

Hayashi, Masahiro, OPTM-8-05

Higuchi, Masato, OPTM-3-04, OPTM-5-01

Hino, Makoto, OPTM-P-07

Hoi, Cheng-Fang, OPTM-P-09

Hong, Feng-Lei, OPTM-1-03

Horprathum, Mati, OPTM-6-03

Hoshino, Tetsuya, OPTM-5-04

Huang, Min, OPTM-P-12

Huang, Ting-Ming, OPTM-P-09

Huang, Kuo-Cheng, OPTM-P-11

lizuka, Yuki, OPTM-6-04

Ishimaru, Ichiro, OPTM-4-03, OPTM-5-03

Ismail, Mohamed, OPTM-8-03

Ismailov, Ismail, OPTM-P-08

Itoh, Masahide, OPTM-5-04

Iwaki, Junya, OPTM-5-03
Jin, Lianhua, OPTM-6-04, OPTM-7-03

Kang, Hanyue, OPTM-4-03, OPTM-5-03

Kawashima, Natsumi, OPTM-4-03, OPTM-5-03

Kemao, Qian, OPTM-2-01

Kim, Daesuk, OPTM-6-02

Kiratiratanapruk, Kantip, OPTM-4-02

Kishimoto, Takumi, OPTM-3-02

Kishore, P., OPTM-P-06

Kitazaki, Tomoya, OPTM-4-03, OPTM-5-03

Ko, Do-Kyeong, OPTM-P-05

Kofman, Jonathan, OPTM-3-01

Kondoh, Eiichi, OPTM-6-04

Kong, Xinxin, OPTM-7-02

Kumar, B., OPTM-P-06

Kumme, R., OPTM-8-03

Kuwano, Ryoichi, OPTM-P-07

Lertvanithphol, Tossaporn, OPTM-6-03

Li, HuanHuan, OPTM-P-02

Li, Shiping, OPTM-2-05

Li, Yang, OPTM-7-02

$\mathrm{Li}$, Ying, OPTM-2-05

Lin, Yu-Hsuan, OPTM-P-1 1

Lin, Jing-Sheuan, OPTM-P-03

Lin, Bin, OPTM-P-01

Liu, Cheng-Yang, OPTM-2-04

Liv, Xinran, OPTM-3-01

Lobach, Ivan, OPTM-P-14

LU, Xiangning, OPTM-P-12

Lv, Qunbo, OPTM-5-05

Ma, Yuzhao, OPTM-8-02

Madokoro, Shuhei, OPTM-5-02

Maeda, Yuki, OPTM-2-02

Matsukuma, Hiraku, OPTM-5-02

Mermoul, A., OPTM-P-13

Metzner, Sebastian, OPTM-8-06

Michihata, Masaki, OPTM-8-01, OPTM-8-05

Mizutani, Yasuhiro, OPTM-4-01, OPTM-8-04

Mizutani, Sora, OPTM-4-03

Morita, Sho, OPTM-P-07

$\mathrm{Na}$, Youngbin, OPTM-P-05

Nakanishi, Hiroki, OPTM-8-04

Nakao, Masaru, OPTM-5-02

Nguyen, Dong, OPTM-3-04

Otani, Yukitoshi, OPTM-2-02, OPTM-6-01, OPTM-6-05, OPTM-P-07

Pei, Linlin, OPTM-5-05

Poletaev, Dmitrii, OPTM-P-08

Poolcharuansin, Phitsanu, OPTM-6-03

Porntheeraphat, Supanit, OPTM-4-02

Prasertsak, Anchalee, OPTM-4-02

Prempree, Panintorn, OPTM-4-02 
Prisyajniuk, Andrey, OPTM-P-08

Quang, Anh, OPTM-3-04

Reithmeier, Eduard, OPTM-3-03

Reuter, Tamara, OPTM-8-06

Roy, Sourabh, OPTM-P-06

Sakurai, Kenji, OPTM-5-04

Seawsakul, Kittikhun, OPTM-6-03

Sevryugin, A., OPTM-P-13

Shankar, M., OPTM-P-06

Sheroshenko, Vladislav, OPTM-P-13

Shibata, Shuhei, OPTM-2-02, OPTM-6-01

Shimizu, Yuki, OPTM-5-02

Shostka, Nataliya, OPTM-P-08

Sinthupinyo, Wasin, OPTM-4-02

Sokolenko, Bogdan, OPTM-P-08

Songsiriritthigul, Prayoon, OPTM-6-03

Sun, Jianying, OPTM-5-05

Takahashi, Satoro, OPTM-8-01, OPTM-8-05

Takamasu, Kiyoshi, OPTM-8-01, OPTM-8-05

Takaya, Yasuhiro, OPTM-4-01, OPTM-8-04

Takeda, Mitsuo, OPTM-1-02

Temniranrat, Pitchayagan, OPTM-4-02

Teng, Li-Wei, OPTM-2-04

Tkachenko, Alina, OPTM-P-14

Toeberg, Stefan, OPTM-3-03

Tokunaga, Tsuyoshi, OPTM-P-07

Trigub, Maxim, OPTM-P-04

Tsai, Hsin-Yi, OPTM-P-1 1

Tutsch, Rainer, OPTM-7-01

Vasnev, Nikolay, OPTM-P-04

Vlasov, Vasiliy, OPTM-P-04

Wang, Cheng-Yu, OPTM-2-04

Wang, Ruisong, OPTM-8-02

Watanabe, Norio, OPTM-5-04

Wei, Dong, OPTM-3-04, OPTM-5-01

Wei, Shuen, OPTM-P-10

Weng, Chun-Jen, OPTM-P-03

Wu, Zhou, OPTM-7-02

Xiangli, Bin, OPTM-7-02

Xiao, Gui, OPTM-P-10

Xing, Shuo, OPTM-2-03

Xiong, Xinglong, OPTM-8-02

$\mathrm{Xu}$, Changda, OPTM-P-02

Yagi, Otoki, OPTM-4-01

Yamamoto, Naoyuki, OPTM-5-03

Yokei, Makoto, OPTM-8-05

Yokoyama, Kotone, OPTM-4-03

Yoshizawa, Toru, OPTM-7-03

Zhang, Zibang, OPTM-2-05

Zhang, Mengyue, OPTM-P-01

Zhang, Wenxi, OPTM-7-02

Zhang, Runan, OPTM-P-10

Zhao, Zheng, OPTM-8-01

Zhong, Jingang, OPTM-2-05

Zhou, Xiang, OPTM-P-02 


\title{
Conference Committee
}

\author{
Conference Chair \\ Takeshi Hatsuzawa, Tokyo Institute of Technology (Japan) \\ Conference Co-chairs
}

Rainer Tutsch, Technische Universität Braunschweig (Germany)

Toru Yoshizawa, Tokyo University of Agriculture and Technology

(Japan)

Conference Program Committee

Masato Aketagawa, Nagaoka University of Technology (Japan)

Yasuhiko Arai, Kansai University (Japan)

Prathan Buranasiri, King Mongkut's Institute of Technology Ladkrabang (Thailand)

Jürgen W. Czarske, TU Dresden (Germany)

Motoharu Fujigaki, University of Fukui (Japan)

Amalia Martínez-García, Centro de Investigaciones en Óptica, A.C. (Mexico)

Satoshi Gonda, National Institute of Advanced Industrial Science and Technology (Japan)

Sen Han, University of Shanghai for Science and Technology (China)

Feng-Lei Hong, Yokohama National University (Japan)

Nathan Hagen, Utsunomiya University (Japan)

Hideki Ina, Canon Inc. (Japan)

Ichiro Ishimaru, Kagawa University (Japan)

Lianhua Jin, University of Yamanashi (Japan)

Daesuk Kim, Chonbuk National University (Korea, Republic of)

Jonathan D. Kofman, University of Waterloo (Canada)

Kazuhide Kamiya, Toyama Prefectural University (Japan)

Qian Kemao, Nanyang Technological University (Singapore)

Fumio Koyama, Tokyo Institute of Technology (Japan)

Ryoichi Kuwano, Hiroshima Institute of Technology (Japan)

Yu-Lung Lo, National Cheng Kung University (Taiwan)

Yasuhiro Mizutani, Osaka University (Japan)

Christian Rembe, Technische Universität Clausthal (Germany)

Yukitoshi Otani, Utsunomiya University (Japan)

Pavel Pavlicek, Palacký University Olomouc (Czech Republic)

Takamasa Suzuki, Niigata University (Japan) 
Satoru Takahashi, The University of Tokyo (Japan)

Toshiyuki Takatsuji, National Institute of Advanced Industrial Science and Technology (Japan)

Toshitaka Wakayama, Saitama Medical University (Japan)

Wei-Chung Wang, National Tsing Hua University (Taiwan)

Gao Wei, Tohoku University (Japan)

Jiangtao Xi, University of Wollongong (Australia)

Hayato Yoshioka, Tokyo Institute of Technology (Japan)

Song Zhang, Purdue University (United States) 


\title{
Towards happy marriage between optics/photonics and AI:
}

\section{A mini tutorial with a historical perspective}

\author{
Mitsuo Takeda* \\ Center for Optical Research and Education (CORE), Utsunomiya University \\ 7-1-2, Yoto, Utsunomiya, Tochigi, 321-8585, Japan
}

\begin{abstract}
Stimulated by the recent revival of interest in artificial intelligence (AI) and neural networks, I will present a mini tutorial review on the past and present efforts that have been made by researchers toward the goal of happy marriage between optics/photonics (OP) and AI. The review will provide a historical perspective through the eyes of an old researcher in the field of optics who experienced the previous high tide of a neural network boom in the 1980s and who has actively participated in the research of optical neuro computing at the time.
\end{abstract}

Keywords: neural network, AI (artificial intelligence), optical computing, inverse problem, optical neuro computing

\section{Introduction}

Many of recent innovations in science and technology have been brought about by cross-disciplinary research. Understanding different fields of sciences provides new insights into one's own field and also can spark ideas for technological innovation. The talk will explain, with examples, how this synergy of knowledge applies also to the fields of optics/photonics (OP) and AI, which, until recently, have developed independently with different disciplines of physical sciences and information sciences.

\section{Historical Analogy}

To get a perspective of the cross-disciplinary research between $\mathrm{OP}$ and $\mathrm{AI}$, we first refer to the historical analogy to the prior example of the successful crossdisciplinary research between $\mathrm{OP}$ and communication engineering (CE). The successful OP-CE integration was achieved by bidirectional technology transfer, namely (1) introduction of OP technologies (laser diodes and optical fibers) to $\mathrm{CE}$, which gave birth to today's broadband communication networks, and (2) introduction of $\mathrm{CE}$ (communication theory) to OP, which resulted in the establishment of holography, heterodyne interferometry, and Fourier optics. Similarly, one can envisage the following scenario for OP-AI integration; (1) introduction *takeda@opt.utsunomiya-u.ac.jp of OP technologies to AI, as exemplified by the research in optical neural networks and neuromorphic photonics, and (2) introduction of AI algorithms to OP, such as application of deep learning to optical sensing and metrology, and new AI-based resolution enhancement in optical imaging, as shown in Figure 1.

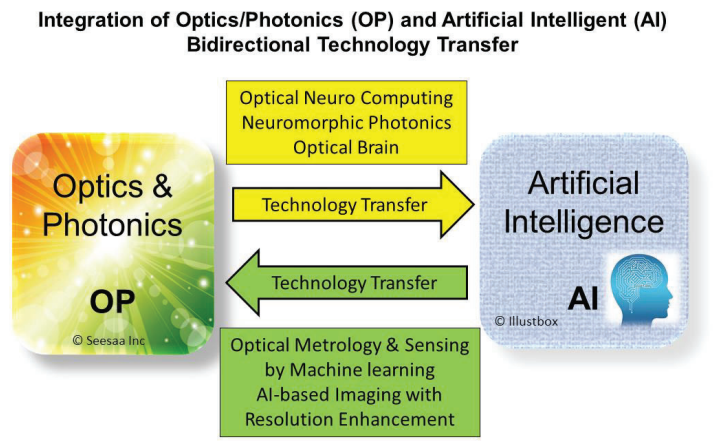

Figure 1. Bidirectional technology transfer for the integration of optics/photonics (OP) and artificial intelligence (AI).

\section{Optical Neuro Computing and}

\section{Neuromorphic Photonics}

Historically optical implementation of neural networks was motivated from the facts that (1) a neural network is an interconnect-intensive system, which requires great 
many product-sum operations for synaptic weight sum calculations, and that (2) a fully parallel optical vector product-sum operation was invented making use of spatial parallelism of optical fields. In the 1980s, various optical neural networks were developed, among which are an optical Hopfield network ${ }^{1}$, an optical Associatron ${ }^{2}$, and an optoelectronic LSI neurochip ${ }^{3}$. While these were based on optical intensity-based implementation with incoherent light, associate memories using coherent complex optical neural fields inside a phase conjugate mirror resonator were also demonstrated ${ }^{4}$. The idea behind the coherent implementation was the philosophy of Haken 5 "Nature computes." The idea of performing parallel computation making use of synergetic dynamics governed by the laws of nature is still being exploited in today's advanced research on a coherent Ising machine. ${ }^{6}$

\section{AI-based Optical Metrology and Imaging}

Various neural network models have been proposed; feedforward nets, feedback (Hopfield) nets, and their combinations and variants. Roughly speaking, the function of a feedforward multilayered net is to establish, through learning, the mapping relation between inputs $\boldsymbol{x}$ and outputs $\boldsymbol{y}$ in such a manner that it represents the statistical rule $\boldsymbol{G}$ hidden in the ensemble of given data; $E=|\boldsymbol{y}-\boldsymbol{G}(\boldsymbol{x} ; \boldsymbol{w}, \boldsymbol{I})|^{2} \Rightarrow$ Minimize with $(\boldsymbol{w}, \boldsymbol{I})$ as variables where $(\boldsymbol{w}, \boldsymbol{I})$ are network parameters known as synaptic weights and biases. We note that this has a close formal similarity to inverse problems in optical metrology and imaging, and also to problems in optical design. As an old yet naïve researcher in optics, I am tempted to pose a question "Can neural networks represent the complex input-output relations of general optical systems that involve complicated nonlinear physical processes?" (see Figure 2) Presently I do not have an answer myself, but partial answers can be found in several examples. Robert et al. ${ }^{7}$ regarded a neural network as a regression method based on a nonlinear model, applied it to the inverse problem of scatterometry in lithography, and successfully detected a micro grating profile. Feng et al. ${ }^{8}$ applied deep learning to fringe analysis for phase detection in 3-D profilometry. Deep learning was also applied to wavefront sensing ${ }^{9}$ without recourse to interferometry, and also to resolution enhancement in microscopy. ${ }^{10} \mathrm{I}$ will review some of these examples in my talk.

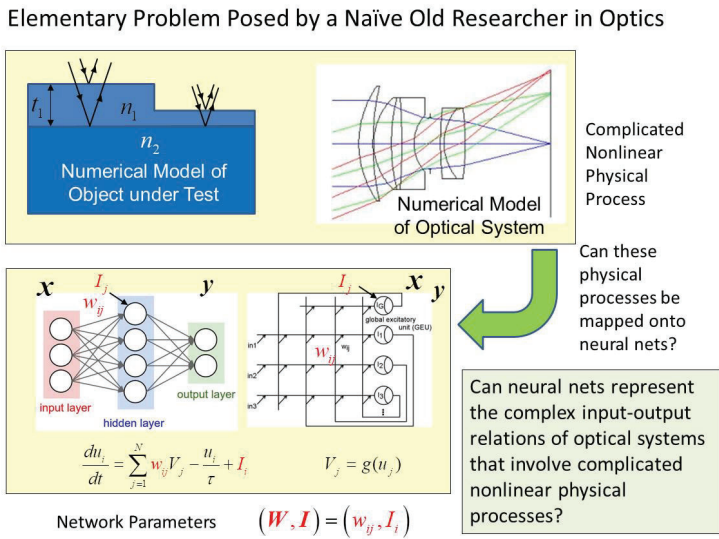

Figure 2. Elementary question posed by an old yet naïve researcher in optics.

\section{References}

[1] Psaltis, D, Farhat, N., "Optical information processing based on an associative-memory model of neural nets with thresholding and feedback," Opt. Lett. 10(2), 98-100 (1985).

[2] Ishikawa, M., Mukohzak, N. Toyoda, H., Suzuki, Y., "Optical associatron: a simple model for optical associative memory," Appl. Opt. 28(2), 291-301 (1989).

[3] Ohta, J. et al., "GaAs/AlGaAs optical synaptic interconnection device for neural networks," Opt. Lett. 14(16), 844-846 (1989).

[4] Takeda, M., Kishigami, T., “Complex neural fields with a Hopfield-like energy function and an analogy to optical fields generated in phase-conjugate resonators," J. Opt. Soc. Am. A, 9(12), 2182-2191 (1992).

[5] Haken, H., [Synergetic Computers and Cognition: A Top-Down Approach to Neural Nets], Springer Verlag, Berlin Heidelberg (1991).

[6] McMahon, P. L. et al., "A fully-programmable 100spin coherent Ising machine with all-to-all connections," Science 0.1126/science.aah5178 (2016).

[7] Robert, S. et al., "Characterization of optical diffraction gratings by use of a neural method," J. Opt. Soc. Am. A, 19(1), 24-32 (2002).

[8] Feng, S. et al., "Fringe pattern analysis using deep learning," arXiv: 1807.02757v1 [eess.IV] 8 Jul. (2018).

[9] Nishizaki, Y. et al., "Deep learning wavefront sensing," Opt. Express, 27(1) 240 -251 (2019).

[10] Rivenson, Y. et al., "Deep learning microscopy," Optica, 4(11) 1437-1443 (2017). 


\title{
Optical frequency metrology with frequency combs and stabilized lasers
}

\author{
Feng-Lei Hong* \\ Department of Physics, Graduate School of Engineering Science, Yokohama National University \\ 79-5 Tokiwadai, Hodogaya-ku, Yokohama 240-8501, Japan \\ *e-mail: hong-fl@ynu.ac.jp
}

\begin{abstract}
Optical frequency combs and frequency-stabilized lasers play important roles not only in the field of precision measurement and standards, but also in basic research of physics. The invention of optical frequency combs has revolutionized the field of optical frequency measurement and further stimulated research on frequency-stabilized lasers. We introduce the recent developments in this field and also the research activities undergoing in our laboratory at the Yokohama National University.
\end{abstract}

Keywords: optical frequency comb, frequency-stabilized laser, dual-comb spectroscopy, astro-comb, interferometric measurement

\section{Introduction}

Optical frequency metrology [1] is of great interest in relation to fundamental science and technologies that support broadband communication networks and the navigation with global positioning systems (GPS). It was demonstrated that mode-locked femtosecond lasers could be used to measure the absolute frequency of an optical frequency standard $[2,3]$. In recent years, erbium-doped fiber based frequency combs (fiber combs) have become widely used owing to their robustness, cost effectiveness, and user friendliness [4]. Frequency-stabilized lasers attract significant interest not only for metrology applications but also for high-resolution spectroscopy. The rapid development of research on optical frequency measurement based on femtosecond combs has stimulated the field of frequency metrology, especially research on high-performance optical frequency standards [5].

In the present paper, we report on the research background of optical frequency combs and frequencystabilized lasers. We have started the development of a narrow-linewidth frequency comb using a compact fiberpigtailed electro-optic modulator, the generation of a broadband visible comb using a PPLN waveguide, and Dual-comb spectroscopy using low repetition rate frequency combs. Recently, we have also started an ultracompact frequency-stabilized laser project. These lasers are useful for various applications, including interferometric measurement.

\section{Research Background}

Figure 1 shows the number of scientific publications related to "optical frequency comb" and "frequencystabilized laser" based on "Web of Science". During the past 10 years, the publications on frequency combs have increase by 3 times. The most cited articles on frequency combs are related to the microresonator-based optical frequency combs. On the other hand, the most cited articles on frequency-stabilized lasers are related to the narrow-linewidth frequency-stabilized lasers.

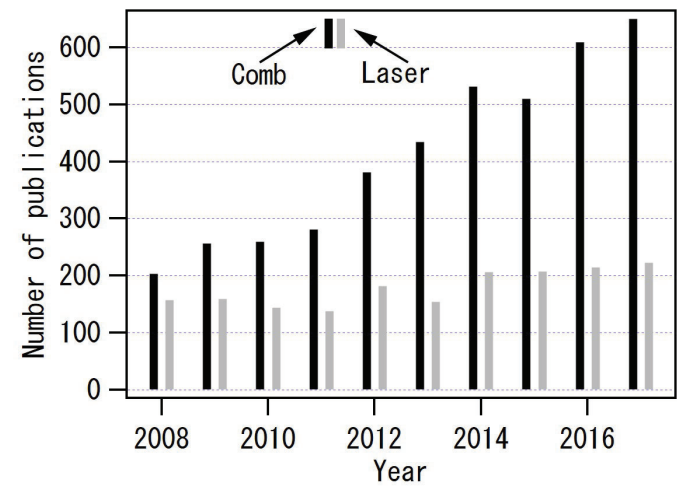

Figure 1. Number of scientific publications investigated based on" Web of Science". 


\section{Development of Frequency Combs}

\section{Development of a narrow-linewidth frequency comb using an electro-optic modulator}

Figure 2 shows a mode-locked Er:fiber laser using an intra-cavity electro-optic modulator (EOM). The laser oscillator employs nonlinear polarization evolution as a mode-locking mechanism. The net dispersion of the oscillator is estimated to be $0.009 \mathrm{ps}^{2}$, and the repetition frequency of the oscillator was about 49.2 MHz. A 15$\mathrm{cm}$-long optical bench with fiber pigtails on both ends is used for loading an EOM, a polarizer and three waveplates. The optical bench is made of Thorlabs FBC1550-FC and FT-38X135. The insertion loss of the optical bench system was less than $2 \mathrm{~dB}$. The EOM is needed for controlling the effective laser cavity length at a large servo bandwidth. We succeeded in phase-locking the mode-locked laser to an optical reference without using a high-voltage amplifier for the EOM.

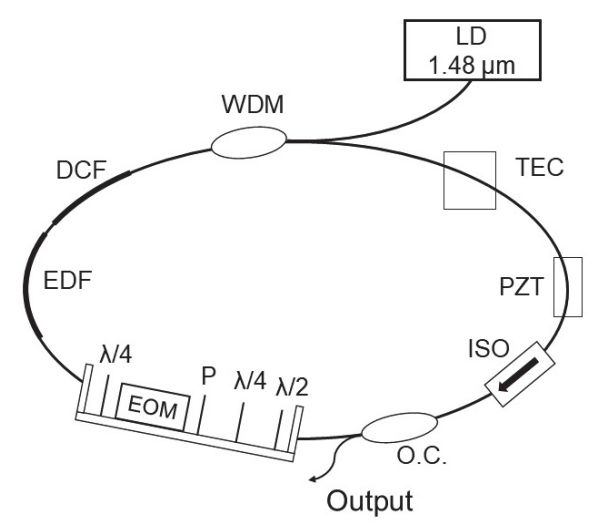

Figure 2. Schematic diagram of the mode-locked Er fiber laserwith a compact optical bench containing an EOM. LD: Laser Diode, WDM: Wavelength Division Multiplexing, DCF: Dispersion Compensation Fiber, EDF: Erbium Doped Fiber, EOM: Electro-Optic Modulator, P: Polarizer, O. C.: Output Coupler, ISO: Isolator, PZT: Piezoelectric Transducer, TEC: Thermoelectric Cooler.

\section{Generation of a broadband visible comb using a PPLN waveguide}

To generate an ultra-broadband optical comb, we used an Er-fiber comb with a repetition rate $\left(\mathrm{f}_{\text {rep }}\right)$ of $107 \mathrm{MHz}$ and a $\mathrm{f}_{\text {ceo }}$ of $10.7 \mathrm{MHz}$. The output pulses from the oscillator are divided into four branches; one of them, which is used for wavelength conversion, is amplified from $1.7 \mathrm{~mW}$ to $116.7 \mathrm{~mW}$ with an Er-doped fiber amplifier (EDFA). Subsequently, the amplified pulse train is spectrally broadened in a highly nonlinear fiber (HNLF) from $1.0 \mu \mathrm{m}$ to at least $1.7 \mu \mathrm{m}$. The output beam from HNLF is then coupled into a WG-PPLN. Figure 3 shows a spectrogram of a broadband comb in the visible region. These results clearly show that the comb spectrum entirely covers the visible region.

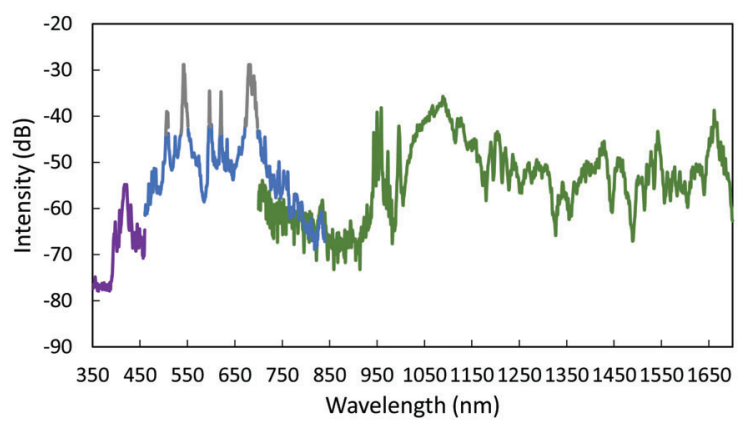

Figure 3. Observed ultra-broad frequency comb spectra from $350 \mathrm{~nm}$ to $1700 \mathrm{~nm}$ in WG-PPLN.

\section{Dual-comb spectroscopy using low repetition rate} frequency combs

Figure 4 shows an absorption spectrum obtained using dual-comb spectroscopy with a low repletion rate of 20 MHz. This is an enlarged graph around the $\mathrm{P}(12)$ line oif the $v_{1}+v_{3}$ band. There are more than 50 comb components across the 1-GHz Doppler broadening. The resolution of the dual-comb spectroscopy increased by a factor of 2.5 to 5 compared to that of a usual frequency comb.

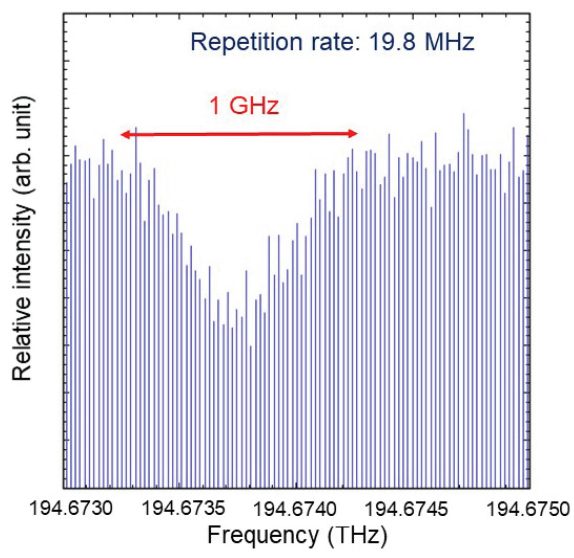

Figure 4. Observed $\mathrm{P}(12)$ line of the $\mathrm{v}_{1}+\mathrm{v}_{3}$ band of acetylene using the low-repetition rate combs. 


\section{Development of Frequency-Stabilized Lasers}

\section{Ultra-compact frequency-stabilized laser}

Figure. 5(a) shows an image of a compact laser module emitting at $531 \mathrm{~nm}$ (QDLaser, QLD0593-3220). The dimensions of the compact laser modules are $20 \mathrm{~mm}$ $\times 6 \mathrm{~mm} \times 4 \mathrm{~mm}$ (length $\times$ width $\times$ thickness). The laser modules consist of a DFB diode laser (DFB-DL) operating in the infrared (IR) region, a semiconductor optical amplifier, and a periodically poled lithium niobate crystal for second harmonic generation (SHG), as shown in Fig. 5(b). We have demonstrated the observation of hyperfine components of iodine transitions using the lowcost coin-sized laser modules based on Doppler-free spectroscopy. Laser frequency stabilization is performed using the observed hyperfine components. We obtained a laser frequency stability of $4.3 \times 10^{-12}$ at an averaging time of $1 \mathrm{~s}$. (a)

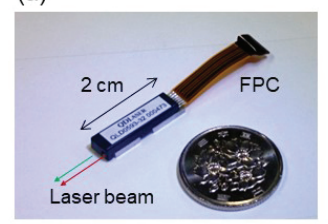

(b)

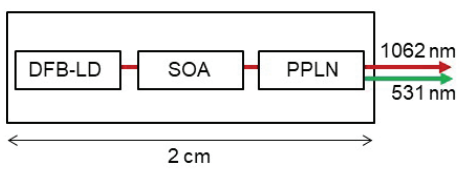

Figure 5. (a) Picture of the compact laser module. (b) Schematic diagram of the compact laser module. DFB-LD: Distributed-Feedback Diode Laser, SOA: Semiconductor Optical Amplifier, PPLN: Periodically Poled Lithium Niobate.

\section{Various frequency-stabilized lasers}

We have developed various frequency-stabilized lasers in our laboratory.

1) Iodine-stabilized $\mathrm{Nd}: \mathrm{YAG}$ lasers

2) 1064-nm narrow-linewidth frequency-stabilized diode lasers

3) 1542-nm narrow-linewidth acetylene-stabilized diode lasers

4) 1062-nm ultra-compact frequency-stabilized diode lasers

5) 399-nm frequency-stabilized external-cavity diode lasers

\section{Conclusions}

It is now an exciting period for researchers working on optical frequency metrology. Rapid developments on optical clocks, frequency combs, and advanced frequency links will open up new possibilities for research ranging from fundamental physics, and astrophysics, to aspects of engineering such as space clocks and the remote sensing of the beat of our earth.

\section{Acknowledgements}

The author is grateful to the group members at YNU, including assistant Prof. K. Yoshii. The author is also grateful to H. Inaba, S. Okubo, D. Akamatsu, T. Kobayashi and other members at NMIJ, AIST. This work was supported by the Japan Society for the Promotion of Science (JSPS) KAKENHI (15H02028, 18H03886, 18H01898) and the Japan Science and Technology Agency (JST) ERATO MINOSHIMA Intelligent Optical Synthesizer (IOS) Project (JPMJER1304).

\section{References}

[1] T. Udem, R. Holzwarth, and T. W. Hänsch, "Optical frequency metrology," Nature 416, 233-237 (2002).

[2] T. Udem, J. Reichert, R. Holzwarth, and T. W. Hänsch, "Absolute optical frequency measurement of the cesium D1 line with a mode-locked laser," Phys. Rev. Lett. 82, 3568-3571 (1999).

[3] D. J. Jones, S. A. Diddams, J. K. Ranka, A. Stentz, R. S. Windeler, J. L. Hall, and S. T. Cundiff, "Carrierenvelope phase control of femtosecond mode-locked lasers and direct optical frequency synthesis," Science 288, 635-639 (2000).

[4] H. Inaba, Y. Daimon, F.-L. Hong, A. Onae, K. Minoshima, T. R. Schibli, H. Matsumoto, M. Hirano, T. Okuno, M. Onishi, and M. Nakazawa, "Long-term measurement of optical frequencies using a simple, robust and low-noise fiber based frequency comb," Opt. Express 14, 5223-5231 (2006).

[5] F.-L. Hong, "Optical frequency standards for time and length applications," Meas. Sci. Technol. 28, 012002 (2017). 


\title{
On Carrier Fringe Pattern Analysis
}

\author{
Qian Kemao \\ School of Computer Science and Engineering, Nanyang Technological University, 639798, \\ Singapore, mkmqian@,ntu.edu.sg
}

\begin{abstract}
Introducing a carrier into fringe patterns enables easy, accurate and robust phase retrieval, and thus has become one of the pillar techniques in optical metrology. The well-known carrier fringe pattern analysis methods include the Fourier transform (FT) method, the spatial phase-shifting (SPS) method, the windowed Fourier transform (WFT) method and the sampling moiré (SM) method. In this paper, the close relationships among these methods will be analyzed and revealed, resulting in a small methodology family.

Keywords: carrier fringe pattern analysis, phase extraction, Fourier transform method, spatial phase-shifting method, windowed Fourier transform method, sampling Moiré method
\end{abstract}




\title{
3D profilometry by projecting polarization pattern
}

\author{
Yuuki Maeda*, Shuhei Shibata, Nathan Hagen, Yukio Otani \\ Center for Optical Research and Education, Utsunomiya University, 7-1-2 Yoto, Utsunomiya, \\ Tochigi. 321-8585. Japan
}

\begin{abstract}
We demonstrate a uniaxial 3D profilometry system that captures a structured linear polarization pattern with a polarization camera having micropolarizers of 4 orientation angles arranged on CCD sensor. The linear polarization pattern is generated by a spatial light modulator (SLM) and a quarter wave plate (QWP) in the optical system. This system can measure 4 different fringe patterns with a phase difference of 90 degrees simultaneously, allowing for faster profilometry measurement than conventional equipment allows. We present experimental results of 3D profilometry using this system.
\end{abstract}

Keywords: profilometry, polarization camera, polarization pattern,

\section{Introduction}

In recent years, technology is growing remarkably and many high precision and small components are being used, increasing the need to measure 3D profilometry with high accuracy and high speed. Non-contact 3D profilometry is particularly needed in order to avoid damaging the object under measurement. Many non-contact methods have been proposed such as moiré and grating projection. [1] However, many of these methods cannot be used within a deep hole or for large step heights due to the use of a triangulation-based method. To solve this problem, it has been proposed to use instead a focusing method, in which the projection system and the imaging system are coaxial. Yoshizawa et al. presented a three-dimensional shape measurement technique using the contrast distribution. [2] However, this technique is a problem that it takes time to project 4 phase-shifted fringe patterns. [3-6]

We propose a uniaxial 3D profilometry system using linear polarization patterns and a polarization camera to enable faster profilometry measurement than conventional equipment allows.

\section{Background}

\section{Linear polarization pattern}

The optical layout for the system is shown in Figure 1. The linear polarization pattern is generated from the liner polarization which is made from the linear polarizer (LP) by a spatial light modulator (SLM) and a quarter wave plate
(QWP). The optical axis of SLM is $0^{\circ}$ and the optical axis of QWP is $45^{\circ}$. SLM provides retardance $\delta(x, y)$ spatially.

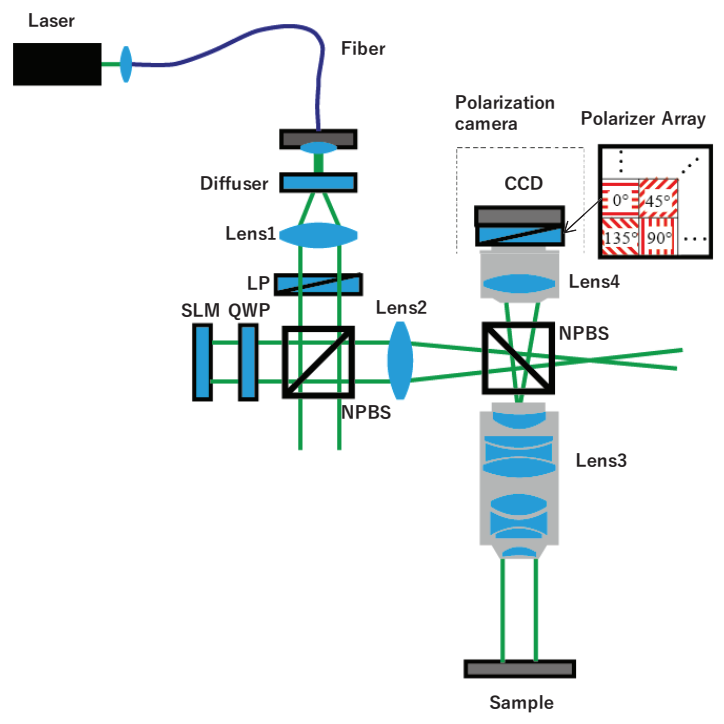

Figure 1. Optical system for 3D profilometry system

By using the Stokes approach, the emission Stokes parameters $S_{\text {out }}$ is given from the incident Stokes parameters $S_{\text {in }}$ and Mueller matrix among the optical polarization components.

$$
\begin{aligned}
& \mathrm{S}_{\text {out }}=\mathrm{Q}_{-45^{\circ}} \cdot \mathrm{S}_{0^{\circ}} \cdot \mathrm{Q}_{45^{\circ}} \cdot \mathrm{P}_{0^{\circ}} \cdot \mathrm{S}_{\text {in }} \\
& S_{\text {out }}=\left[\begin{array}{c}
1 \\
\cos \{\delta(x, y)\} \\
\sin \{\delta(x, y)\} \\
0
\end{array}\right]
\end{aligned}
$$

where $Q_{\theta}, S_{\theta}, P_{\theta}$ represent Mueller matrix of QWP, SL 
M, LP and $\theta$ represents optical axis.

From Stokes parameters $\mathrm{S}_{\text {out }}$, the azimuthal direction of linear polarization is provided by retardance $\delta(x, y)$ value of SLM. If this retardance is controlled spatially, linear polarization pattern rotated spatially is obtained.

\section{Contrast distribution}

The intensity detected by the CCD sensor of the polarization camera can be described as:

$\mathrm{I}(\mathrm{x}, \mathrm{y})=\frac{1}{2}\{1+\gamma(\mathrm{x}, \mathrm{y}) \cos (\delta(\mathrm{x}, \mathrm{y})-2 \varphi(\mathrm{x}, \mathrm{y}))\}$

for $\varphi(x, y)=0^{\circ}, 45^{\circ}, 90^{\circ}, 135^{\circ}$ the orientation angles of the linear polarizer on each pixel of the polarization camera and $\gamma(x, y)$ the modulation contrast. The contrast is calculated from the detected image by the 4-step phase shifting technique.

$\gamma(x, y)=\frac{2 \sqrt{\left(I_{0}(x, y)-I_{2}(x, y)\right)^{2}+\left(I_{1}(x, y)-I_{3}(x, y)\right)^{2}}}{I_{0}(x, y)+I_{1}(x, y)+I_{2}(x, y)+I_{3}(x, y)}$

where $\mathrm{I}_{0}(\mathrm{x}, \mathrm{y}), \mathrm{I}_{1}(\mathrm{x}, \mathrm{y}), \mathrm{I}_{2}(\mathrm{x}, \mathrm{y})$, and $\mathrm{I}_{3}(\mathrm{x}, \mathrm{y})$ represent the intensity $\mathrm{I}(x, y)$ of $\varphi(x, y)=0^{\circ}, 45^{\circ}, 90^{\circ}$ and $135^{\circ}$.

There are two methods for measuring the height from the contrast. The first method obtains the height from the contrast of a sample spatially by referring the height against contrast measured in advance. Therefore, this first method enables snapshot 3D profilometry measurement.

Wilson et al presented the best focus position equal the maximum value of contrast of a sample. [7] Therefore, the second method obtains the height from the best focus potion of a sample by measuring contrast spatially while varying the height of sample.

\section{Results and Discussion}

The result of measuring the contrast while varying the height of the planar mirror is shown in Figure 2(a). The solid line shows the contrast fitted with a Gaussian function. As you can see, the contrast distribution is able to fit with a Gaussian function. The result of the height calculated from the contrast against the height obtained from a micrometer is shown in Figure 2(b). As you can see, the height is directly proportional to the theoretical height in the range of $4 \mathrm{~mm}-10 \mathrm{~mm}$. In this case, the measurement is performed in the range. Measurement accuracy of the height obtained from this result is $150 \mu \mathrm{m}$. Therefore, measurement accuracy is not good.
The result of measuring a 1951 USAF test target as a sample using the first method are shown in Figure 3. While the measurement obtains the correct overall threedimensional shape of the target, some errors are encountered due to the low of reflectivity. But it was confirmed that enables snapshot 3D profilometry measurement.

(a)

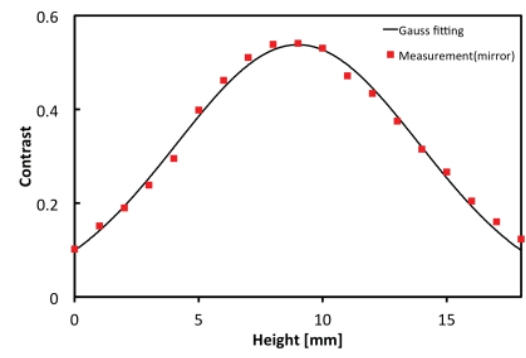

(b)

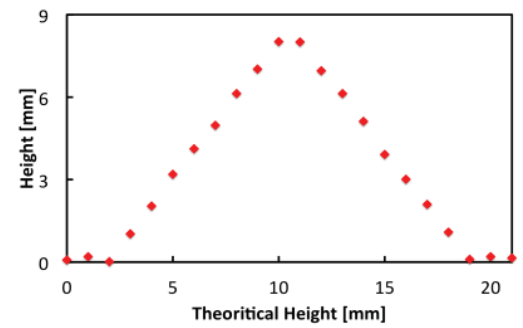

Figure 2. Measurement result of the planar mirror

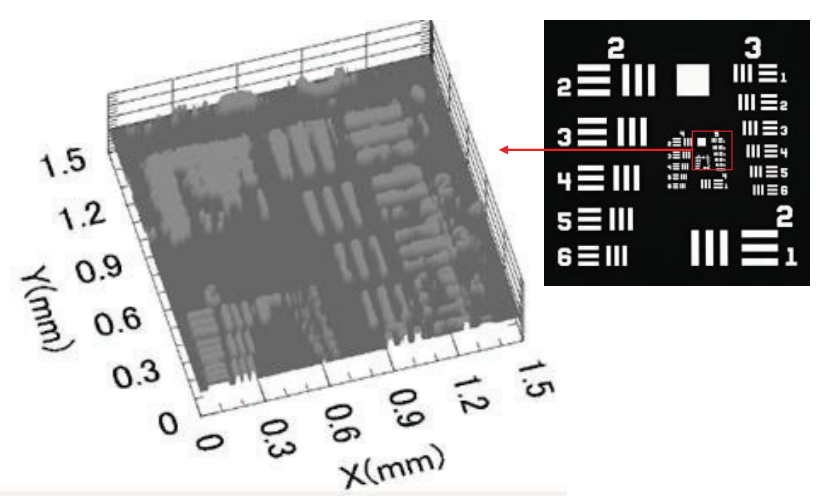

Figure 3. Measurement result of the 1951 USAF test target

The result of measuring many kinds of samples using the second method is shown in Figure 4. The position of the maximum value of the contrast is the same regardless of the kinds of samples. The measurement accuracy of each 
sample is shown in Table 1, from which we can see that the second method is much more accurate than the first method. We used the surface of a U.S. 10 cent coin as a sample. The result of measuring the sample using the second method is shown in Figure 5, confirming that highprecision three-dimensional shape measurement is possible.

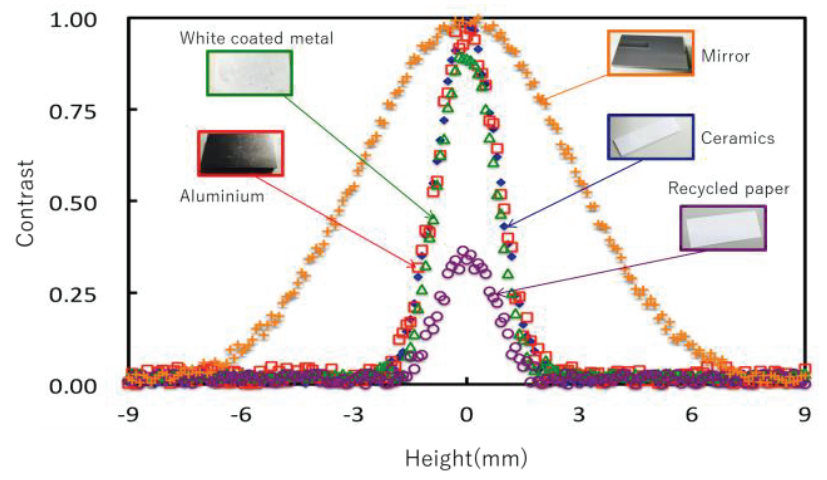

Figure 4. Measurement result of many kinds of samples

Table 1. Measurement accuracy of each sample

\begin{tabular}{|l|l|l|l|l|l|}
\hline Sample & mirror & Ceramics & $\begin{array}{l}\text { White } \\
\text { coated } \\
\text { metal }\end{array}$ & Aluminium & $\begin{array}{l}\text { Recycled } \\
\text { paper }\end{array}$ \\
\hline Accuracy & $\pm 6 \mu \mathrm{m}$ & $\pm 3 \mu \mathrm{m}$ & $\pm 5 \mu \mathrm{m}$ & $\pm 4 \mu \mathrm{m}$ & $\pm 4 \mu \mathrm{m}$ \\
\hline
\end{tabular}
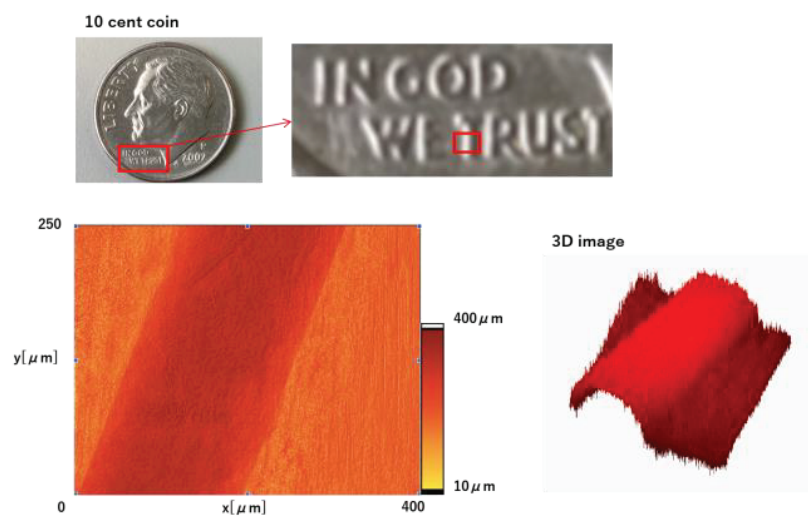

Figure 5. Results of measuring a 10 cent coin as a sample

\section{Conclusion}

In conclusion, the present study has demonstrated a uniaxial 3D profilometry system using spatially linear polarization patterns and a polarization camera. The first method enables snapshot measurement of 3D profiles, but it has a problem of measurement accuracy. The second method is much more accurate than the first method, but it needs a lot of measurement to obtain the peak of contrast distribution. Therefore, this method has a problem of measurement speed. Further studies are needed in order to 3D profilometry with high accuracy and high speed.

\section{References}

[1] Fumio Kobayashi, Yukitoshi Otani, Toru Yoshizawa: Projection type of moiré topography with frequency modulation technique using liquid crystal digital gratings, Opt. Review, Vol. 19, No 3(2012) 154-158

[2] Yukitoshi Otani, Fumio Kobayashi, Yasuhiro Mizutani, Toru Yoshizawa: Three-dimensional profilometry based on focus method by projecting LC grating pattern, Proc. SPIE 743210 (2009).

[3] Y. Xu, S. Zhang, Uniaxial three-dimensional shape measurement with projector defocusing, Opt. Eng., 51 (2) (2012), 023604

[4] Geliztle A. Parra Escamilla, Fumio Kobayashi, Yukitoshi Otani: Three-Dimensional Surface Measurement based on the Projected Defocused Pattern Technique Using Imaging Fiber Optics, Opt. Comm., 390 (2017) 57-60

[5] Y. Mizutani, R. Kuwano, Y. Otani, N. Umeda, T. Yoshizawa: Three-dimensional shape measurement using focus method by using liquid crystal grating and liquid varifocus lens, Proc. SPIE 60000J (2005)

[6] Yukitoshi Otani, Fumio Kobayashi, Yasuhiro Mizutani, Toru Yoshizawa: Three-dimensional profilometry based on focus method by projecting LC grating pattern, Proc. SPIE, 7432, 10(2009).

[7] M. A. A. Neil, R. Juskaitis, and T. Wilson: Method of obtaining optical sectioning by using structured light in a conventional microscope, Opt. Lett. (1997) vol. 22, no. 24, 1905-1907 


\title{
Self-correction of phase errors induced by projector nonlinearity in phase- shifting fringe projection profilometry
}

\author{
Shuo Xing, Hongwei Guo* \\ Laboratory of Applied Optics and Metrology, Department of Precision Mechanical Engineering, \\ Shanghai University, Shanghai 200444, China
}

\begin{abstract}
Self-correction method is presented for removing, without a photometric calibration, the projector nonlinearity errors from a phase map or a couple of phase maps having different frequencies, thus improving the accuracy of fringe projection profilometry.
\end{abstract}

Keywords: projector nonlinearity correction, phase measurement, fringe analysis, phase maps

\section{Introduction}

In fringe projection profilometry, the projector nonlinearity is a main factor decreasing the measurement accuracy. It makes fringes non-sinusoidal thus inducing ripple-like artifacts on the calculated phase maps and, further, on the reconstructed object shapes [1]. Many efforts have been made for solving this problem. Typically, a photometric calibration can be performed for determining the projector nonlinearity curve for compensating for the phase errors actively. Instead, the passive technique uses a look-up table of phase errors versus phase values for correcting these errors. With these methods, a calibration must be implemented in advance, so they may fail in correcting the phase errors induced by the time-variant projector nonlinearity [2] This paper presents a self-correction method for removing the phase errors from a single phase map or a couple of phase maps having different frequencies. This method works without a prior calibration.

\section{Projector nonlinearity}

In phase-shifting fringe projection profilometry, a projector is used for casting sinusoidal fringe patterns on the measured object surface, and the camera captures the deformed fringe patterns. One of the captured patterns is

$$
I_{k}(x, y)=A(x, y) \cos [\phi(x, y)+2 \pi k / K]+B(x, y)
$$

where $(x, y)$ denote the pixel coordinates of a point on the imaging plane of the camera. $A(x, y)$ is the modulation and $B(x, y)$ is the background intensity. $2 k \pi / K$ is an added phase shift with $k=0,1, \ldots, K-1$. From these patterns, the wrapped phases are estimated by [3]

$$
\psi(x, y)=-\arctan \left[\frac{\sum_{k=0}^{K-1} I_{k}(x, y) \sin (2 \pi k / K)}{\sum_{k=0}^{K-1} I_{k}(x, y) \cos (2 \pi k / K)}\right]
$$

By implementing phase unwrapping to $\psi(x, y)$, we have its unwrapped phase map $\Psi(x, y)$.

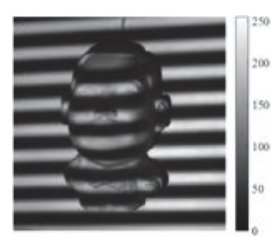

(a)

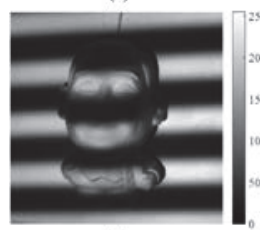

(d)

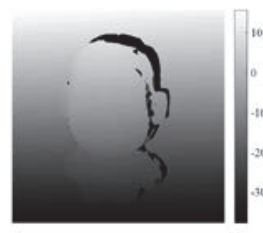

(b)

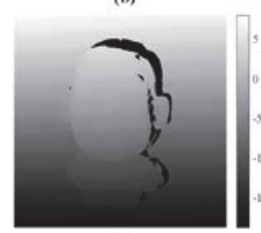

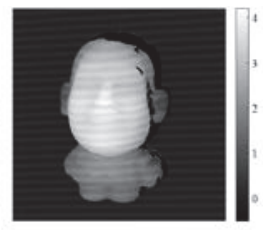

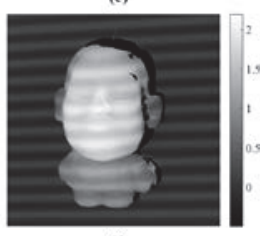

Figure 1. Phase measuring. In each row, the panels show, from left to right, a fringe pattern, its unwrapped phase map, and the phase map without carrier. The fringe pattern in the second row has a frequency halving the one in the first row. The colorbars have the unit of radian.

In the presence of the projector nonlinearity, the unwrapped phase contains errors induced by the projector nonlinearity. According to our analysis in [4], these phase errors has a form of 


$$
\begin{aligned}
\varepsilon(x, y) & =\Psi(x, y)-\Phi(x, y) \\
& \approx \xi_{1} \sin [K \Phi(x, y)]+\xi_{2} \sin [2 K \Phi(x, y)] \\
& +\xi_{3} \sin [3 K \Phi(x, y)]+\cdots
\end{aligned}
$$

We find from Eq. (3) that the phase error induced by the projector nonlinearity depends on the number of phase shifts, having multiplied frequencies higher than the fringe frequencies. We take Fig. 1 as an example. In it, two sequences of fringe patterns having different frequencies are captured, with the number of phase shifts being three; the size of each fringe patterns is $512 \times 512$ pixels; and the projector was not calibrated. The left column shows the first frame of each pattern sequence. The middle column gives the unwrapped phase maps. By removing the carrier, the ripple-like artifacts become observable as we see on the right column.

\section{Error coefficient estimation from a couple of phase maps having different frequencies}

From Eq. (3), we know that if the error coefficients are determined, the phase error can be removed. In multifrequency fringe projection profilometry, at least two phase maps having different frequencies are recovered, making it possible to determine and eliminate the error terms [5]. Here, we denote $\Psi_{H}$ and $\Psi_{L}$ as two unwrapped phase maps corresponding to the fringe frequencies $f_{H}$ and $f_{L}$, respectively. Note that $\Psi_{H}$ and $\Psi_{L}$ are function of $(x, y)$. For each pixel, we have a pair of equations like

$$
\left\{\begin{array}{c}
\Psi_{H}-\Phi_{H}=\xi_{1} \sin \left(K \Phi_{H}\right)+\xi_{2} \sin \left(2 K \Phi_{H}\right)+\cdots \\
\Psi_{L}-\frac{f_{L}}{f_{H}} \Phi_{H}=\xi_{1} \sin \left(K \frac{f_{L}}{f_{H}} \Phi_{H}\right)+\xi_{2} \sin \left(2 K \frac{f_{L}}{f_{H}} \Phi_{H}\right)+\cdots
\end{array}\right.
$$

where $\Phi_{H}$ is the real phase corresponding to the fringe frequency $f_{H}$.

In practice, the errors may have infinite terms, but their amplitudes decline quickly as their orders become high. We reasonably truncate the error function and keep the first three terms. Because the equations are nonlinear, we have to solve it using an iterative algorithm in the least squares sense. Here we use the calculated phases $\Psi_{H}$ as the initial values of $\Phi_{H}$. The error coefficients estimated from Fig. 1are $\xi=\{-0.1551,-0.0095,-0.0019\}$.

\section{Error coefficient estimation from a single phase} map
If only a single phase map is available, it is difficult to estimate the error coefficients. For doing it, we apply a Gaussian low-pass filter to the unwrapped phase in Fig. 1(b), and the smoothed phase map is shown in Fig. 2(a). Its version without carrier is given in Fig. 2(b), from which it is evident that the ripple-like artifacts induced by the projector nonlinearity have been smoothed out. Simultaneously, the edges and details on the phase map are severely blurred, and large errors appear at its boundaries. Fig. 2(c) shows the differences between Fig. 2(a) and Fig. 1(b). These phase differences are mainly composed of the artifacts caused by the projector nonlinearity.

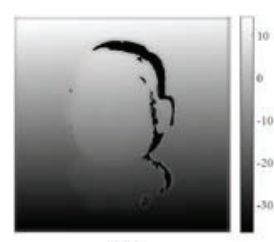

(a)

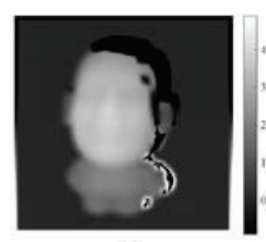

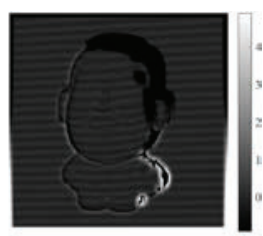

Figure 2. (a) The smoothed phase map by using Gaussian filter. (b) The smoothed phase map without carrier. (c) The difference between (a) and Fig. 1(b).

The values in these figures have a unit of radian.

According to our analysis in [6], the phase error is a function of phase value. The error coefficients can be estimated by fitting the relations between the values from Fig. 2(c) and Fig. 2(b), viz.

$$
\begin{aligned}
\sum_{l=1}^{L} \xi_{l}^{(i+1)} & \sin [l K \bar{\Psi}(x, y)]=\Psi(x, y)-\bar{\Psi}(x, y), \\
& \text { for }(x, y) \text { satisfying }\left|\Delta^{(i)}(x, y)\right| \leq 3 \sigma_{\Delta}^{(i)}
\end{aligned}
$$

where $\bar{\Psi}$ denotes the smoothed phase map. The superscripts in brackets denote the numbers of iterations; $\Delta^{(i)}(x, y)$ is the departure of a point from the central curve. In this iterative procedure, we use the 3-sigma criterion for excluding the outliers caused by the spatial filter. Using this method, the error coefficients estimated from Fig. 2 are $\xi=\{-0.1415,-0.0095,-0.0012\}$.

\section{Phase error correction}

When the coefficients of the error function are obtained, we can correct the nonlinearity-induced phase errors in a pointwise way through the following fixed-point iterations: 


$$
\Phi^{(j+1)}(x, y)=\Psi(x, y)-\sum_{l=1}^{L} \xi_{l} \sin \left[l K \Phi^{(j)}(x, y)\right]
$$

Figures. 3(a) and 3(b) show the corrected phase maps using the error coefficients estimated from a couple of phase maps in Fig. 1, and from the single phase map in Fig. 2, respectively. In these results, the ripple-like artifacts induced by the projector nonlinearity have been removed.
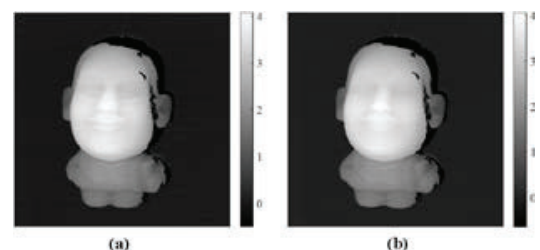

Figure 3. (a) The phase map with its nonlinearityinduced errors having been removed from the couple of phase maps in Fig. 1. (b) The corrected phase map from the single phase map in Fig. 2. The values in these figures have a unit of radian.

\section{Conclusion}

This paper presented a self-correction method that allows us to remove the errors induced by the projector nonlinearity from a single phase map or from a couple of phase maps having different frequencies. This method offers some advantages over the exist ones. For example, it does not require a prior calibration, and hence it is applicable when the projector nonlinearity varies with time. Moreover, it is independent of a special model or an assumption about the projector nonlinearity, thus being more general in practical applications.

\section{References}

[1] H. Guo, H. He, and M. Chen. 2004. Gamma correction for digital fringe projection profilometry. Appl. Opt. 43, 2906-2914.

[2] B. Li, Y. Wang, J. Dai, W. Lohry, and S. Zhang. 2014. Some recent advances on superfast 3D shape measurement with digital binary defocusing techniques. Opt. Lasers Eng. 54, 236-246.

[3] J. H. Bruning, D. R. Herriott, J. E. Gallagher, D. P. Rosenfeld, A. D. White, and D. J. Brangccio. 1974 Digital wavefront measuring interferometer for testing optical surfaces and lenses. Appl. Opt. 13, 2693-2703.
[4] F. Lü, S. Xing, and H. Guo. 2017. Self-correction of projector nonlinearity in phase-shifting fringe projection profilometry. Appl. Opt. 56, 7204-7216.

[5] S. Xing and H. Guo. 2018. Correction of projector nonlinearity in multi-frequency phase-shifting fringe projection profilometry. Opt. Express. 26, 1627716291.

[6] S. Xing and H. Guo. 2019. Directly recognizing and removing the projector nonlinearity errors from a phase map in phase-shifting fringe projection profilometry. Opt. Commun. 435, 212-220. 


\title{
High-speed 3D surface measurement of rear lamp housing by automatic digital fringe projection system*
}

\author{
Cheng-Yang Liu*a, Cheng-Yu Wang ${ }^{\mathrm{b}}$, Li-Wei Teng ${ }^{\mathrm{b}}$ \\ ${ }^{a}$ Department of Biomedical Engineering, National Yang-Ming University, Taipei City, Taiwan \\ ${ }^{b}$ Department of Mechanical and Electro-Mechanical Engineering, Tamkang University, New Taipei \\ City, Taiwan
}

\begin{abstract}
Digital fringe projection method is widely used in industrial applications with high-speed measurement and high resolution. In this study, a fully automatic high-speed digital fringe projection system is presented to profile 3D surface characteristic of rear lamp housing of vehicle. The structured light with fringe pattern is used to be the light source in the measurement system and is projected by a digital light processing projector. The distorted fringe patterns from the surface of lamp housing are captured by the digital camera. The absolute phase maps are calculated by using phase-shifting and quality guided path unwrapping algorithm. A complete 3D feature of lamp housing is obtained by our system. We achieved simultaneous phase acquisition, reconstruction and $3 \mathrm{D}$ exhibition at a speed of $0.5 \mathrm{~s}$. This system can provide a high accuracy and real-time 3D surface measurement for the automobile industry.
\end{abstract}

Keywords: lamp housing, fringe projection, structured light

\section{Introduction}

According to modern digital technology, a real-time three-dimensional (3-D) surface system is a very significant method in many fields including machine manufacture, medical applications and automobile industry [1-13]. Fringe projection technique has become the most popular 3-D shape acquisition method. The fringe projection system classically uses a digital projector to project fringe patterns onto object surfaces, a digital camera to capture the distorted fringe patterns, and a computer to calculate the phase maps and reconstruct the 3-D shape. The primary processes for 3-D shape reconstruction contain phase-shifting calculation, phaseunwrapping calculation and phase to real dimension conversion. Three-step phase-shifting calculation is typically exercised in the fringe projection system. However, three-step phase-shifting method is less efficient and time consuming. Because monochromatic fringe patterns take a long time for capturing and calculation, we are interested in 3-D reconstruction with high-speed for fringe projection system. For this reason, the high-speed digital fringe projection system is presented for 3-D surface measurement.

*cyliu66@ym.edu.tw; phone 886-2-28267020
Single-shot fringe projection measurements are insensitive to signal noises because only one image needs to be captured. The single-shot fringe projection methods can be generally classified into discrete and continuous coding processes [14-20]. The discrete coding patterns present a digital shape with the same intensity value and the shape dimension determines from the intensity of the measured object surface. The image resolution in this case is low since the discrete code occupies several pixels. The obtained 3-D data for measuring complex shape are inaccurate. The continuous coding patterns show a smooth profile where every pixel has a singular codeword on color. Therefore, the obtained 3-D data are dense surface reconstruction. The phase-shifting calculations are widely utilized since the measurement has high accuracy and high speed. We have to investigate phaseshifting algorithms in order to obtain absolute phase data for high-accuracy 3-D reconstruction.

In this study, a high-speed digital fringe projection system is proposed to measure 3-D features of the rear lamp housing. The light source used in this system is structured light with phase-shifting fringe patterns. A projector with digital light processing (DLP) is employed as light source to project fringe patterns onto object surface. The images of distorted fringe pattern are 
captured by a digital camera The multi-step phaseshifting and quality guided path unwrapping calculations are used to compute the value of absolute phase map. The detecting angle of the digital camera is controlled by using a motorized stage. Finally, a complete 3-D surface of the rear lamp housing is obtained by our system. We have successfully accomplished simultaneous phase calculation, 3-D reconstruction and exhibition at a speed of $0.5 \mathrm{~s}$. This results can provide a high-accuracy and real-time 3-D shape measurement for automobile industry.

\section{Principle of the system}

Figure 1 shows the schematic diagram of automatic digital fringe projection system. The automatic fringe projection system includes the following several parts: generation of multi-step phase-shifting fringe pattern, acquisition of deformed fringe pattern, calculations of wrapped and unwrapped phase, and conversion from absolute phase to real size. This system consists of a DLP projector, a digital camera, a motorized rotation stage and an industrial computer. The sinusoidal fringe patterns are generated by using a computer code and projected onto the object surface by the projector. The digital camera is used to acquire the deformed fringe patterns. The phase information is extracted from the captured patterns which has to be unwrapped for surface reconstruction. The unwrapped phase values are calculated by calibration method for the relationship between the absolute phase value and real size.

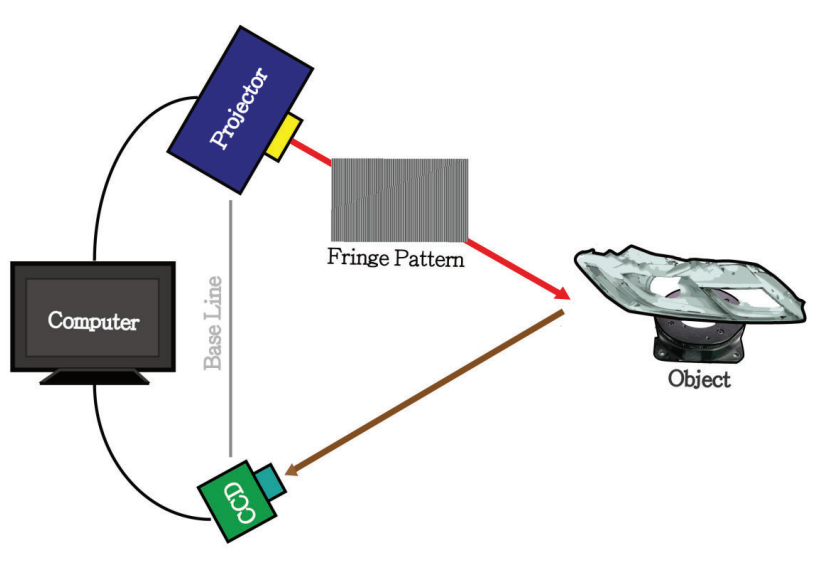

Figure 1. Schematic diagram of automatic digital fringe projection system.
The phase information is obtained from deformed fringe patterns. Multi-step phase-shifting calculations are extensively employed in optical 3-D surface measurement due to its advantageous properties. In this case, three phase-shifting algorithms are utilized to calculate the wrapped phase maps including three-step, four-step, fivestep and seven-step algorithms. The three-step phaseshifting algorithm is preferable for real-time 3-D surface measurement because the minimum numbers of fringe patterns are required for calculations. The phase shift between three fringe patterns is selected as $2 \pi / 3$. Three fringe patterns are applied for this algorithm whose fringe intensities can be expressed as:

$$
\begin{aligned}
& I_{1}=I^{\prime}+I^{\prime \prime} \cos \left(\phi-\frac{2 \pi}{3}\right) \\
& I_{2}=I^{\prime}+I^{\prime \prime} \cos (\phi) \\
& I_{3}=I^{\prime}+I^{\prime \prime} \cos \left(\phi+\frac{2 \pi}{3}\right)
\end{aligned}
$$

where the average intensity is $I^{\prime}$, the intensity modulation is $I^{\prime \prime}$, the phase information is $\phi$.

The phase map can be obtained by solving Eq. (1) as:

$$
\phi=\tan ^{-1}\left[\frac{\sqrt{3}\left(I_{1}-I_{3}\right)}{2 I_{2}-I_{1}-I_{3}}\right]
$$

The seven-step phase-shifting algorithm is preferable for high-accuracy measurement because the maximum numbers of fringe patterns are required for calculations. The phase shift between seven fringe patterns is $\pi / 2$. The intensities of seven fringe patterns are expressed as:

$$
\begin{aligned}
& I_{1}=I^{\prime}+I^{\prime \prime} \cos \left[\phi-\frac{3 \pi}{2}\right] \\
& I_{2}=I^{\prime}+I^{\prime \prime} \cos [\phi-\pi] \\
& I_{3}=I^{\prime}+I^{\prime \prime} \cos \left[\phi-\frac{\pi}{2}\right] \\
& I_{4}=I^{\prime}+I^{\prime \prime} \cos [\phi] \\
& I_{5}=I^{\prime}+I^{\prime \prime} \cos \left[\phi+\frac{\pi}{2}\right] \\
& I_{6}=I^{\prime}+I^{\prime \prime} \cos [\phi+\pi] \\
& I_{7}=I^{\prime}+I^{\prime \prime} \cos \left[\phi+\frac{3 \pi}{2}\right]
\end{aligned}
$$

The intensity of light source and fringe modulation for seven fringe patterns are assumed the same value. The phase $\phi$ can be retrieved by the following equation:

$$
\phi=\tan ^{-1}\left[\frac{3 I_{3}+I_{7}-I_{1}-3 I_{5}}{4 I_{4}-2 I_{2}-2 I_{6}}\right]
$$


In Eqs. (2) and (4), the phase value is a range of $-\pi$ to $+\pi$. Therefore, the phase unwrapping calculation is required to obtain continuous phase information. The quality guided path algorithm is used in the unwrapping calculation. The highest quality pixels are unwrapped with the highest reliability. The lowest quality pixels with the lowest reliability are considered to prevent the error circulation in the phase map. The unwrapping path is determined by the pixel reliability. The quality guided path algorithm is suitable to calculate phase value from complex surface features. In order to obtain absolute phase value, the standard gauge block is used as references to convert the relative phase to absolute phase. Once the digital fringe projection system is calibrated, the coordinates of real sizes can be obtained from the absolute phase value. Figure 2 shows the operation interface of automatic digital fringe projection system. All algorithms and system control are programed in the MATLAB $^{\circledR}$ code. The insert in the upper left corner of Fig. 2 is the function menu of our automatic system.

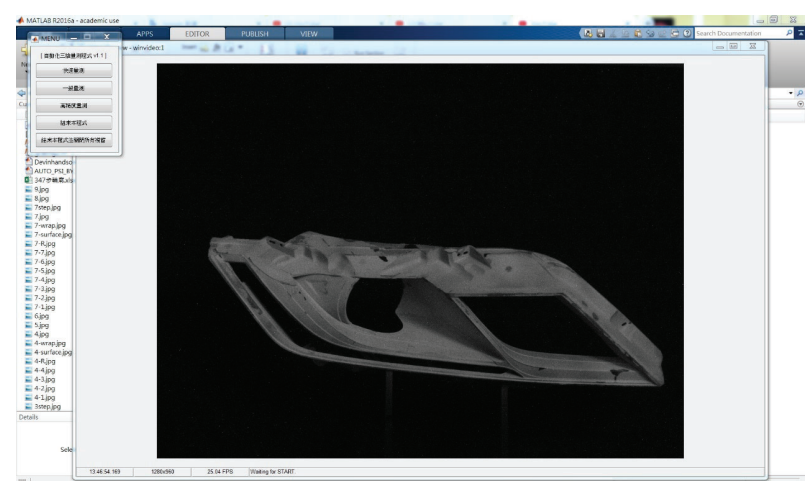

Figure 2. Operation interface of automatic digital fringe projection system.

\section{Results and Discussion}

For high-speed 3-D surface measurement, a structured light is used by encoding three, four, and seven fringe patterns. The fast image switching is reached by the projection mechanism of the DLP technology. The different fringe patterns are projected sequentially which will capture separately by digital camera. Figure 3 shows the captured three-step fringe patterns projected on the surface of rear lamp housing. Figure 4 shows the seven fringe patterns projected on the housing surface.

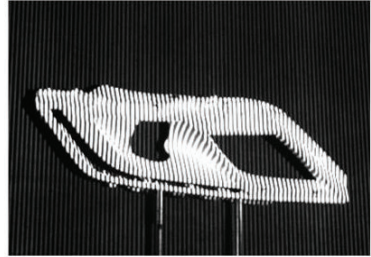

(a)

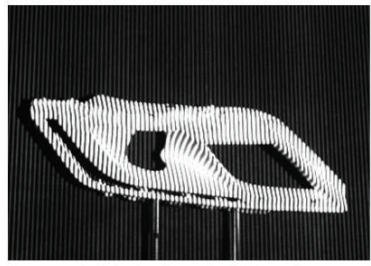

(c)

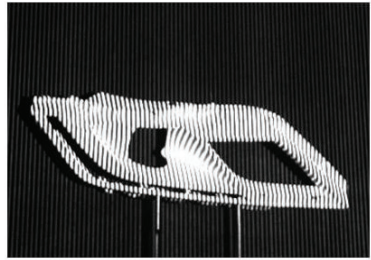

(b)

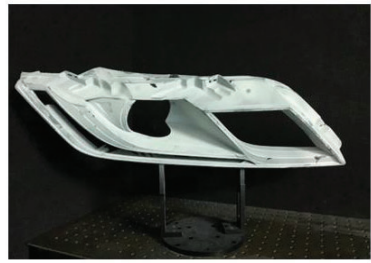

(d)
Figure 3. Three-step fringe patterns projected on the surface of rear lamp housing. The insert in the bottom right is the picture rear lamp housing.
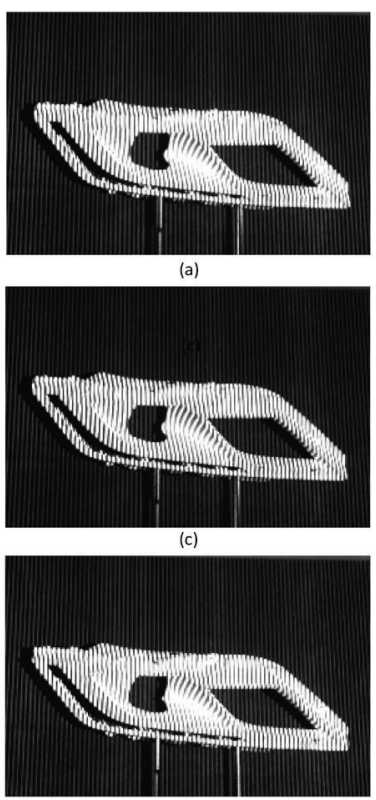

(e)

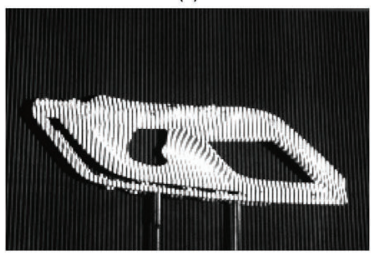

(g)

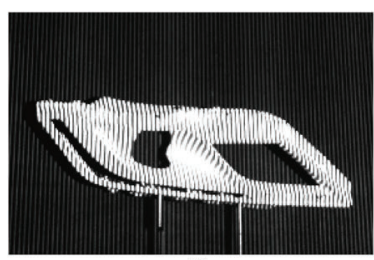

(b)
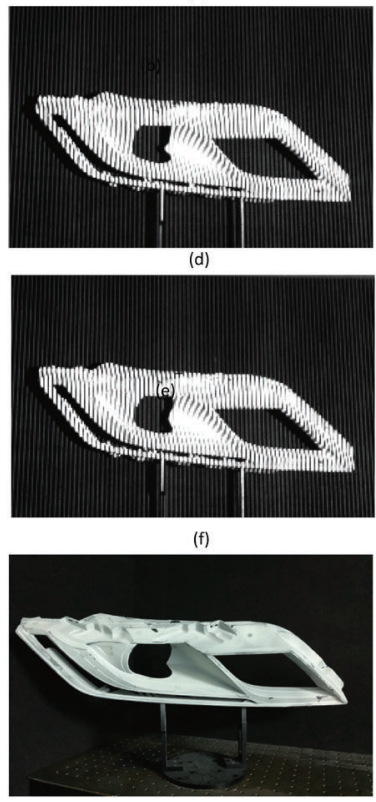

(h)
Figure 4. Seven-step fringe patterns projected on the surface of rear lamp housing. The insert in the bottom right is the picture rear lamp housing. 


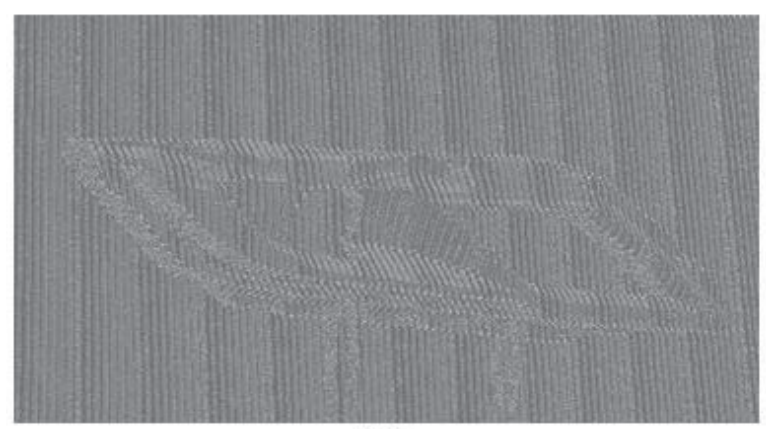

(a)

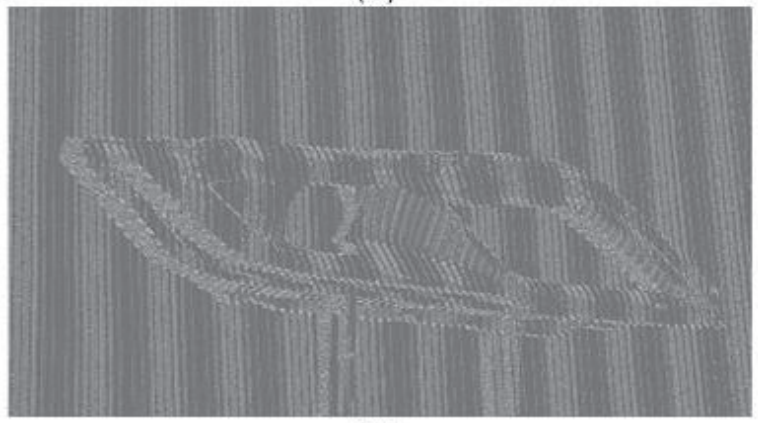

(b)

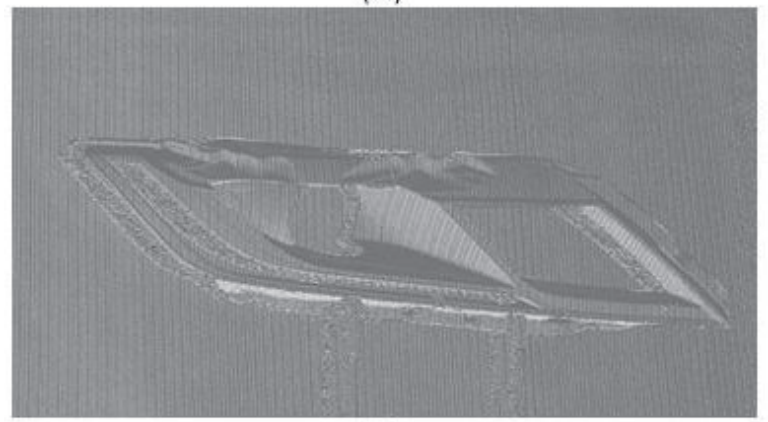

(c)

Figure 5. Absolute phase maps of the rear lamp housing at different phase-shifting calculations: (a) three-step, (b) four-step, and (c) seven-step.

The distorted phase-shifted fringe patterns are used to reconstruct one 3-D surface through phase wrapping, phase unwrapping and phase to real size conversion steps. The calculation process of these deformed fringe patterns by the proposed program gives us the information of 3-D surface. Applying three-step, four-step, and seven-step phase-shifting algorithms to these fringe patterns, the wrapped phase map are obtained. These images imply that the surface change strongly contributes to the phase information. Figure 5 shows the Absolute phase maps of the rear lamp housing at different phase-shifting calculations. Using the proposed digital fringe projection system, lamp housing is captured and processed to obtain the 3-D representation. Figure 6 shows 3-D representation of the rear lamp housing. The experimental results clearly show that the proposed system based on fringe projection technique correctly acquires the 3-D surface of rear lamp housing.

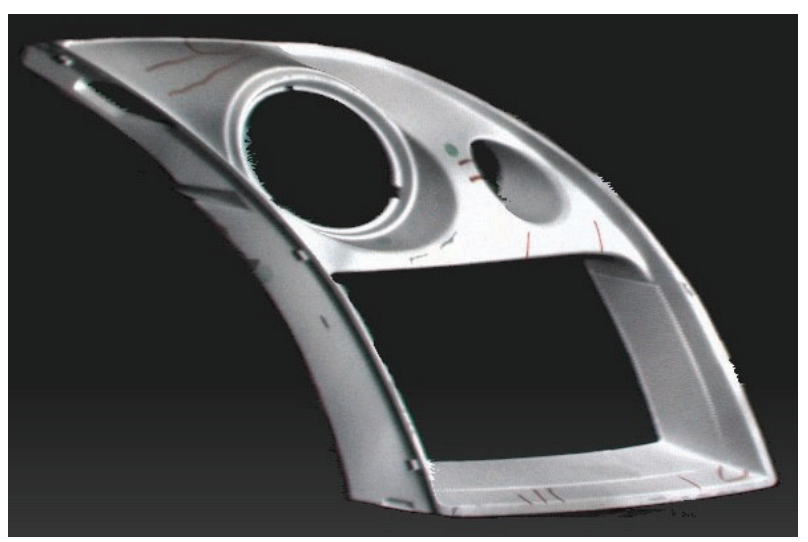

Figure 6. 3-D representation of the rear lamp housing.

\section{Conclusion}

In this study, a high-speed digital fringe projection system is proposed to measure 3-D surface of the rear lamp housing. The light source used in this system is structured light with different fringe patterns. A DLP projector is used as light source to project structured light onto the surface of lamp housing. The distorted fringe pattern images are captured by the digital camera. The multi-step phase-shifting and quality guided path unwrapping algorithms are used to calculate the phase map. The detecting angle of the camera is controlled by using a motorized stage. Finally, a complete 3-D surface of rear lamp housing is obtained by our system. We have achieved simultaneous phase acquisition, surface reconstruction and exhibition at a speed of $0.5 \mathrm{~s}$. The experimental results may provide a high-accuracy and real-time 3-D surface system for automobile industry.

\section{Acknowledgment}

The authors would like to appreciate the partial financial support from the Ministry of Science and Technology in Taiwan with the project number of MOST 107-2221-E-010-020. 


\section{References}

[1] Gorthi, S. and Rastogi, P., "Fringe projection techniques: whither we are?," Opt. Lasers Eng. 48, 133-140 (2010).

[2] Zhang, S., "Recent progresses on real-time 3D shape measurement using digital fringe projection techniques,” Opt. Lasers Eng. 48, 149-158 (2010).

[3] Ma, S., Quan, C., Zhu, R. and Tay, C., "Investigation of phase error correction for digital sinusoidal phaseshifting fringe projection profilometry," Opt. Lasers Eng. 50, 1107-1118 (2012).

[4] Zuo, C., Chen, Q., Gu, G., Feng, S., Feng, F., Li, R. and Shen, G., "High-speed three-dimensional shape measurement for dynamic scenes using bi-frequency tripolar pulse-width-modulation fringe projection," Opt. Lasers Eng. 51, 953-960 (2013).

[5] Feng, S., Zhang, Y., Chen, Q., Zuo, C., Li, R. and Shen, G., "General solution for high dynamic range threedimensional shape measurement using the fringe projection technique," Opt. Lasers Eng. 59, 56-71 (2014).

[6] Nguyen, H., Nguyen, D., Wang, Z., Kieu, H. and Le, M., "Real-time, high-accuracy 3D imaging and shape measurement," Appl. Opt. 54, A9-A17 (2015).

[7] Wang, H., Kemao, Q. and Soon, S., "Valid point detection in fringe projection profilometry," Opt. Express 23, 7535-7549 (2015).

[8] Liu, T., Zhou, C., Si, S., Li, H. and Lei, Z., "Improved differential 3D shape retrieval," Opt. Lasers Eng. 73, 143-149 (2015).

[9] Liu, C. and Yen, T., "Digital multi-step phase-shifting profilometry for three-dimensional ballscrew surface imaging," Opt. Laser Technol. 79, 115-123 (2016).

[10]Dai, M., Yang, F., Liu, C. and He X., "A dualfrequency fringe projection three-dimensional shape measurement system using a DLP 3D projector," Opt. Commun. 382, 294-301 (2017).

[11]Parra Escamill, G., Kobayashi, F. and Otani, Y., "Three-dimensional surface measurement based on the projected defocused pattern technique using imaging fiber optics," Opt. Commun. 390, 57-60 (2017).

[12] Chen, C., Gao, N., Wang, X. and Zhang, Z., “Adaptive projection intensity adjustment for avoiding saturation in three-dimensional shape measurement," Opt. Commun. 410, 694-702 (2018).
[13] Yu, S., Zhang, J., Yu, X., Sun, X., Wu, H. and Liu, X., "3D measurement using combined Gray code and dual-frequency phase-shifting approach," Opt. Commun. 413, 283-290 (2018).

[14]Koninckx, T. and Van Gool, L., "Real-time range acquisition by adaptive structured light," IEEE Trans. Pattern Anal. Mach. Intell. 28, 432-445 (2006).

[15]Chen, S., Li, Y., Guan, Q. and Xiao, G., "Real-time three-dimensional surface measurement by color encoded light projection," Appl. Phys. Lett. 89, 111108 (2006).

[16]Qian, K., Fu, Y., Liu, Q., Seah, H. and Asundi, A., "Generalized three-dimensional windowed Fourier transform for fringe analysis," Opt. Lett. 31, 21212123 (2006).

[17]Chen, S., Li, Y. and Zhang, J., "Vision processing for realtime 3-D data acquisition based on coded structured light," IEEE Trans. Image Process. 17, 167176 (2008).

[18] Salvi, J., Fernandez, S., Pribanic, T. and Llado X., "A state of the art in structured light patterns for surface profilometry," Pattern Recognit. 43, 2666-2680 (2010).

[19]Flores, J., Muñoz, A., Ordoñes, S., Garcia-Torales, G. and Ferraric, J., "Color-fringe pattern profilometry using an efficient iterative algorithm," Opt. Commun. 391, 88-93 (2017).

[20]Flores, J., Ayubi, G., Martino, M., Castillo, O. and Ferrari, J., "3D-shape of objects with straight linemotion by simultaneous projection of color coded patterns," Opt. Commun. 414, 185-190 (2018). 


\title{
Response function measurement in photovoltaic devices with sinusoidal structured illumination
}

\author{
Zibang Zhang ${ }^{\mathrm{a}}$, Qiwen Deng ${ }^{\mathrm{a}}$, Jingang Zhong ${ }^{\mathrm{a}^{*}}$, Shiping $\mathrm{Li}^{\mathrm{a}}$, and Ying $\mathrm{Li}^{\mathrm{b}}$ \\ ${ }^{a}$ Department of Optoelectronic Engineering, Jinan University, Guangzhou 510632, China \\ ${ }^{\mathrm{b}}$ Pre-university, Jinan University, Guangzhou 510610, China
}

\begin{abstract}
It is important to understand the performance and aging mechanisms in photovoltaic devices. However, the commercially available method, laser beam induced current, is too slow for high-throughput measurements, because it uses a laser spot for raster scan. This paper presents the principle and demonstration of a new measurement method which uses structured illumination instead of raster scan. When a photovoltaic device is under structured illumination, the output signal is the sum of the local current response. We propose to use sinusoidal structured illumination to acquire the Fourier transform of the response function. The sinusoidal structured illumination is generated by using a spatial light modulator. As the response function has a sparse representation in Fourier domain, the presented method potentially enables accurate and efficient response function measurement in photovoltaic devices.
\end{abstract}

Kevwords: photovoltaic device. reponse function measurement. sinusoidal structured illumination. Fourier transform

\section{Introduction}

It is important to understand the performance and aging mechanisms in photovoltaic (PV) devices. However, commercially available method, laser beam induced current [1-4], is too slow for high-throughput measurements, because it uses a laser spot for raster scan.

This paper presents the principle and demonstration of a new measurement method which uses structured illumination instead of raster scan. When a photovoltaic device is under structured illumination, the output signal is the sum of the local current response. Particularly, if sinusoidal patterns are used for structured illumination, the output signal will be equivalent to a Fourier coefficient of the entire response function of the PV device. By changing the spatial frequency and the initial phase of the sinusoidal structured illumination, the Fourier transform of the response function can be obtained. The response function can be derived by simply applying an inverse Fourier transform. As the response function can be sparsely represented in the Fourier domain, undersampling the Fourier transform of the response function allows for significant reduction of measurements. Thus, the presented method enables fast response function measurements in PV devices. Moreover, as sinusoidal structured illumination is a kind of global illumination, the input light intensity is much higher than that of single-point scanning by laser beam induced current method. Thus, the presented method allows the PV device to produce a much larger photocurrent with a consequent noise reduction due to an improved signal-to-noise ratio. In short, the presented method potentially enables accurate and efficient response function measurement in photovoltaic devices.

\section{Principle}

Assuming that the response function of a PV device is presented by $R(x, y)$, the output current $I$ of the device under illumination of $P(x, \mathrm{y})$ :

$$
I=\iint_{\Omega} R(x, y) P(x, \mathrm{y}) \mathrm{d} x \mathrm{~d} y+\varepsilon
$$

where $\Omega$ denotes the active area of the device and $\varepsilon$ denotes the current resulted from dark noise. Our goal is to recover the response function from the output current. Specifically, we can choose an appropriate basis set for structured illumination so as to encode the spatial information of the response function into the output current. Here, we propose to use sinusoidal structured illumination, considering response function of PV devices has a sparsed representation in Fourier space. The sinusoidal intensity patterns can generated by using a spatial light modulator (such as, DMD or LCoS). Each 
sinusoidal intensity pattern $P_{\phi}\left(x, y ; f_{x}, f_{y}\right)$ is

characterized by its spatial frequency pair $\left(f_{x}, f_{y}\right)$ and

initial phase $\phi$ :

$$
P_{\phi}\left(x, y ; f_{x}, f_{y}\right)=a+b \cdot \cos \left(2 \pi f_{x} x+2 \pi f_{y} y+\phi\right) .
$$

By substituting Eq. (2) into Eq. (1), we find that the output current is mathematically the inner product of the sinusoidal intensity pattern and the response function of the PV device. As sinusoidal patterns are actually the basis of Fourier transform, it implies that the measurement is equivalent to a Fourier coefficient $\tilde{R}\left(f_{x}, f_{y}\right)$. However, the measurement will be affected by the noise term $\varepsilon$. In order to eliminate the noise, we propose to use phase-shifting strategy. Specifically, four sinusoidal intensity patterns with the same spatial frequency but different initial phases are used for illumination sequentially. The phase-shifting illumination are denoted by $P_{0}, P_{\pi / 2}, P_{\pi}$, and $P_{3 \pi / 2}$, respectively. And the corresponding measurements are represented by $I_{0}\left(f_{x}, f_{y}\right), I_{\pi / 2}\left(f_{x}, f_{y}\right), I_{\pi}\left(f_{x}, f_{y}\right)$, and $I_{3 \pi / 2}\left(f_{x}, f_{y}\right)$, respectively. With the measurements, the Fourier coefficient corresponding to spatial frequency $\left(f_{x}, f_{y}\right)$ can be derived by

$$
\tilde{R}\left(f_{x}, f_{y}\right)=\left[\bar{I}_{\pi}\left(f_{x}, f_{y}\right)-\bar{I}_{0}\left(f_{x}, f_{y}\right)\right]+\mathrm{j} \cdot\left[\bar{I}_{3 \pi / 2}\left(f_{x}, f_{y}\right)-\bar{I}_{\pi / 2}\left(f_{x}, f_{y}\right)\right] .
$$

By changing the spatial frequency, the Fourier transform of the response function can be obtained. The response function can be derived by simply applying an inverse Fourier transform.

\section{Experiment}

In our experiment, we measured the response function of a photodiode (Hamamatsu S1227-1010BR). The sinusoidal intensity patterns were shown on the computer display in turn. The patterns were projected onto the active area of the target photodiode through a thin lens. The display used (SAMSUNG LS22D300NY) was lightemitted-diode back-illuminated. The size of the display is with the size of 21.5 inches $(268.11 \mathrm{~mm}(H) \times 476.64 \mathrm{~mm}$ $(V)$ ). The pixel size of the display is $0.24825 \mathrm{~mm}(H) \times$ $0.24825 \mathrm{~mm}(V)$. The display operated in $1920(H) \times$ 1080( $V$ )-pixel mode. The display switched patterns every 0.3 seconds.

The size of the generated sinusoidal intensity pattern is $127 \times 127$ points. The display allows the patterns to be displayed in gray, red, green and blue, respectively by the screen. Usin $\$ 5$ the monochrome patterns for illumination allows us to measure the response of the PV device at a different waveband. We used a spectrometer (Ocean Optics USB4000) for measurement and derived the optical spectra shown in Figure 1. According to the figure, the central wavelength of the red, green, and blue illumination patterns are $610 \mathrm{~nm}, 547.8 \mathrm{~nm}$, and $450.4 \mathrm{~nm}$, respectively. The figure also shows that green patterns have largest intensity, blue patterns have narrowest spectral bandwidth.

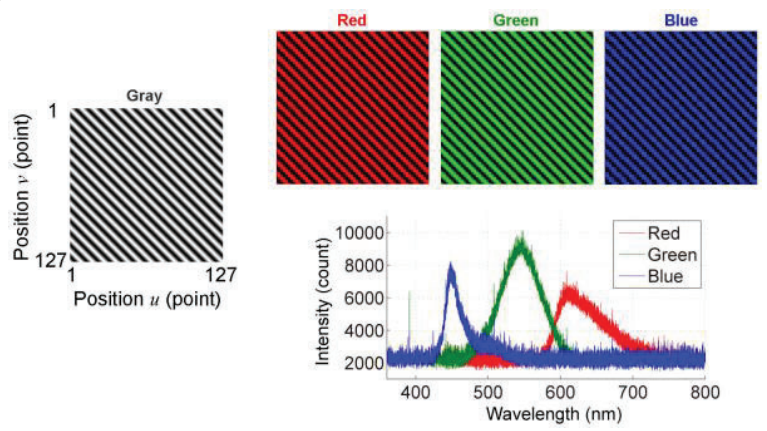

Figure 1. Example of gray sinusoidal intensity pattern (left). Red, green, and blue sinusoidal intensity patterns (top-right). The spectra of the utilized display for red, green, and blue patterns display (bottom-right).

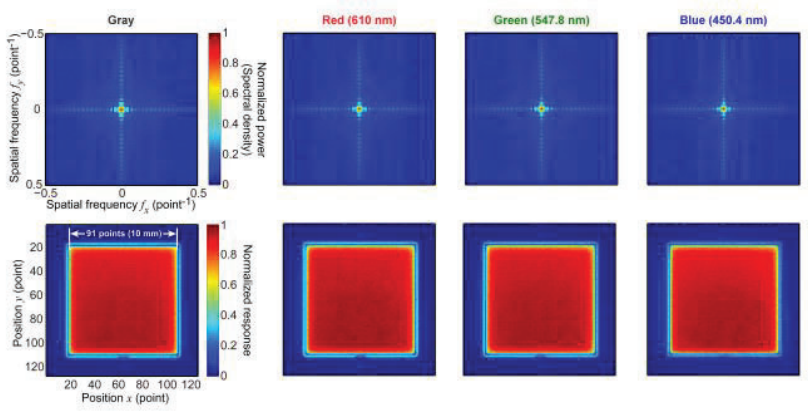

Figure 2. Reconstructed Fourier spectra (top) and response functions (bottom) for gray, red, green, and blue (from left to right), respectively. 
As the Fourier spectra shown in Figure 2, the energy of the response function is mainly concentrated at and around the origin of the Fourier domain. It implies that the response function has a sparsed representation in the Fourier domain. As such, we can only sample the lowfrequency region of the Fourier transform to reduce the number of measurements. As the results shown in Fig. 2, the square response area with fine structures is clearly reconstructed. Fig. 3 presents a $3 \mathrm{D}$ representation of the reconstructed response function for the photodiode under green sinusoidal structured illumination.

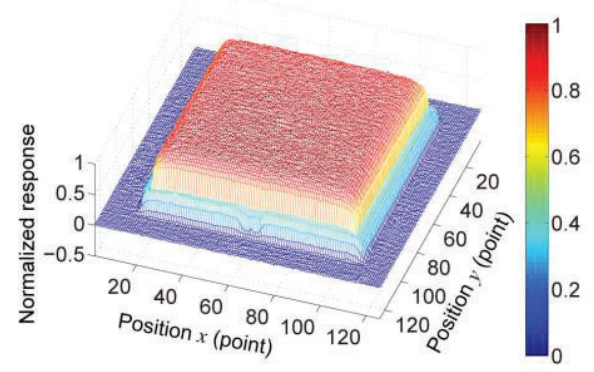

Figure 3. 3D representation of the measured response function of the photodiode under green sinusoidal structured illumination.

\section{Conclusion}

We propose to use sinusoidal structured illumination generated by a spatial light modulator so as to acquire the Fourier transform of the response function. As the response function has a sparse representation in Fourier domain, the presented method potentially enables accurate and efficient response function measurement in photovoltaic devices.

\section{References}

Martín. J., C. Fernández-Lorenzo, J. A. Poce-Fatou and R. Alcántara. 2004. A versatile computer-controlled highresolution LBIC system. Prog. Photovolt. Res. Appl 12(4):283-295.

Carstensen. J., G. Popkirov, J. Bahr and H. Föll. 2003. CELLO: An advanced LBIC measurement technique for solar cell local characterization. Solar Energy Mater. Solar Cells 76(4):599-611.

Vorasayan. P., T. R. Betts, A. N. Tiwari and R. Gottschalg. 2009. Multi-laser LBIC system for thin film PV module characterisation. Solar Energy Mater. Solar
Cells 93(6-7):917-921.

Cossutta. H., K. Taretto and M. Troviano. 2014. Low-cost system for micrometer-resolution solar cell characterization by light beam-induced current mapping. Meas. Sci. Technol 25(10):105801. 


\title{
Innovations in Structured Light Methods and Optical Metrology
}

\author{
Jonathan Kofman, Xinran Liu \\ Department of Systems Design Engineering, University of Waterloo, 200 University Avenue W, \\ Waterloo, ON N2L 3G1, Canada \\ jkofman@uwaterloo.ca,xinran.liu@uwaterloo.ca
}

\begin{abstract}
The increasing demand for greater resolution, accuracy, and measurement speed, in three dimensional (3D) non-contact surface-shape measurement, and the challenges of real-world applications of measuring highly reflective, moving, and deforming surfaces has led to innovations in structured light methods and optical metrology, from laser based to full-field fringe projection methods.

\section{Introduction}

Optical methods have been used for non-contact three dimensional (3D) surface-shape measurement in various applications ranging from part inspection and reverse engineering in manufacturing, to human body scanning for biomedical and entertainment applications [1-4]. Stereovision systems employing two or more cameras can provide high accuracy calibration and $3 \mathrm{D}$ point reconstruction, if the correspondences of pixels between the camera images can be accurately determined. Determination of correspondences is difficult without texture in geometry (roughness, edges, corners) or intensity.
\end{abstract}

\section{Structured lighting}

Structured light methods avoid the correspondence problem by projecting a light pattern onto the object surface, capturing an image of the light pattern at an angle, and performing 3D point reconstruction using triangulation $[1,2]$. Single-dot projection scanning has been used for simple static part inspection, and machine vision based on laser-line projection has been useful for industrial on-line part inspection.

Structured-light techniques evolved with multiple-line projection techniques to obtain more surface-shape information by a single captured image. This permitted faster 3D surface measurement and enabled 3D surface-shape measurement by a hand-held 3D scanner without requiring sensor pose tracking or surface markers [5]. This also permitted robot-mounted-sensor applications where the sensor is moving.

\section{Full-field optical metrology}

In response to an increasing demand for greater resolution and accuracy, and higher measurement speed, structured-light techniques evolved to full-field fringe projection methods, which compute $3 \mathrm{D}$ point coordinates on a surface at every camera pixel. Fringe projection phase-shifting profilometry (PSP) uses projection of multiple phase-shifted patterns, capture of corresponding images, and phase computation. Earlier methods of 3D reconstruction employed phase-to-height mapping, determined in a calibration process by computing phase at multiple positions of a known, often planar, calibration object. In addition to the high resolution, the method have the advantages of being insensitive to ambient light and handling smooth freeform surfaces without surface texture. 
Hybrid optical metrology methods combine high resolution phase computation on textureless surfaces by fringe projection, use of the calculated phase values to determine correspondences at every camera pixel for stereovision, and high-accuracy stereovision calibration and 3D reconstruction, to improve surface measurement accuracy with simpler calibration.

\section{Challenges of real-world applications}

Further innovations in fringe projection methods have been necessary for the challenges of real-world applications. Novel adaptive fringe projection methods have been developed for measurement of highly reflective specular surfaces and surfaces of varying reflectivity [6,7]. Fringe-projection and moiré surface-measurement methods also aim to meet the demand for higher measurement speed without loss of accuracy, for moving and deforming surfaces. In addition to high-speed hardware approaches [8], innovative techniques have been developed as an alternative, using fewer projected patterns and captured images, in order to maintain low cost. These techniques include specially encoding fringe patterns, new approaches in pixel-wise wavelength refinement for single-frame digital phase-shifting moiré, and geometric constraints $[9,10]$ to avoid ambiguity in determining correct phase from periodic phase maps. Pixel-wise methods further permit fast processing using parallel computing for real-time applications.

\section{References}

[1] Blais F. Review of 20 years of range sensor development. J Electronic Imag 2004, 13 (1), 231-240.

[2] Geng J. Structured-light 3D surface imaging: a tutorial. Adv Opt Photon 2011, 3, 128-160.

[3] Bakirman T, Gumusay MU, Reis HC, Selbesoglu MO, Yosmaoglu S, Yaras MC, et al. Comparison of low cost 3D structured light scanners for face modeling. Appl Opt 2017, 56, 985-992.

[4] Öberg K, Kofman J, Karlsson A, Lindström B, Sigblad G. The CAPOD system - A Scandinavian CAD/CAM system for prosthetic sockets. J Prosthet Orthot 1989, 1(3), 139-148.

[5] Kofman J, Borribanbunpotkat K. Hand-held 3D scanner for surface-shape measurement without sensor pose tracking or surface markers, Virtual Phys Prototyp 2014, 9(2), 81-95.

[6] Zhang S, Yau ST. High dynamic range scanning technique. Opt Eng 2009, 48(3), 033604.

[7] Li D, Kofman J. Adaptive fringe-pattern projection for image saturation avoidance in 3D surface-shape measurement. Opt Express 2014, 22(8), 9887-9901.

[8] Hyun J-S, Chiu GT-C, Zhang S. High-speed and high-accuracy 3D surface measurement using a mechanical projector. Opt Express 2018, 26, 1474-1487.

[9] Tao T, Chen Q, Feng S, Hu Y, Da J, Zuo C. High-precision real-time 3D shape measurement using a bi-frequency scheme and multi-view system. Appl Opt 2017, 56(13), 3646-3653.

[10] Liu X, Kofman J. High-frequency background modulation fringe patterns based on a fringe-wavelength geometry-constraint model for 3D surface-shape measurement. Opt Express 2017, 25, 16618-16628.

\section{Acknowledgement}

This research was supported by the Natural Sciences and Engineering Research Council of Canada (NSERC); University of Waterloo; and China Scholarship Council (CSC). 


\title{
Development of Handy Type Full-color and Real-time 3D Measurement System Using Linear LED Device*
}

\author{
Takumi Kishimoto*a $^{\mathrm{a}}$, Motoharu Fujigaki ${ }^{\mathrm{a}}$ \\ ${ }^{a}$ Human and Artificial Intelligent Systems, Graduate School of Engineering, University of Fukui, \\ 3-9-1 Bunkyo, Fukui-shi, Fukui 910-8507, Japan
}

\begin{abstract}
In recent years, demands for non-contact 3D shape measurement are increased in many fields. In particular, large demands for product inspection in the factory are increased. In this research, a handy type 3D shape measurement device using linear LED devices is developed. In addition, full-color measurement was realized with using RGB LEDs on the linear LED device. Furthermore, measurement was speeding up and real-time measurement was realized by multithreading of the program.
\end{abstract}

Keywords: 3D shape measurement, real-time measurement, full-color measurement, multithreading, whole-space tabulation method (WSTM), light-source-stepping method (LSSM), linear LED device

\section{Introduction}

Compact and fast 3D shape measurement system is required in many fields such as an industrial field. In our laboratory, a 3D measurement method based on a grating projection method using a whole-space tabulation method (WSTM) [1] was proposed. It is possible to measure 3D coordinates at high speed by using a relation table between the phase and the coordinates at each pixel. A light-source-stepping method (LSSM) [2] was also proposed, high-speed phase shift can be achieved by switching the lighting position on a linear LED device.

In this paper, a handy type 3D shape measurement device using linear LED devices is developed. Moreover, color information of the surface of an object can be obtained with using RGB LEDs on the linear LED device. Furthermore, measurement was speeding up and real-time measurement was realized by multithreading of the program.

\section{Measurement device}

The measurement system consists of a camera, a projector, a control board of linear LED device, and a battery. Figure 1 shows the measurement device. The projector was downsized by using linear LED devices. A handy 3D shape measurement device was developed. Figure 2 shows a photograph of the linear LED device.

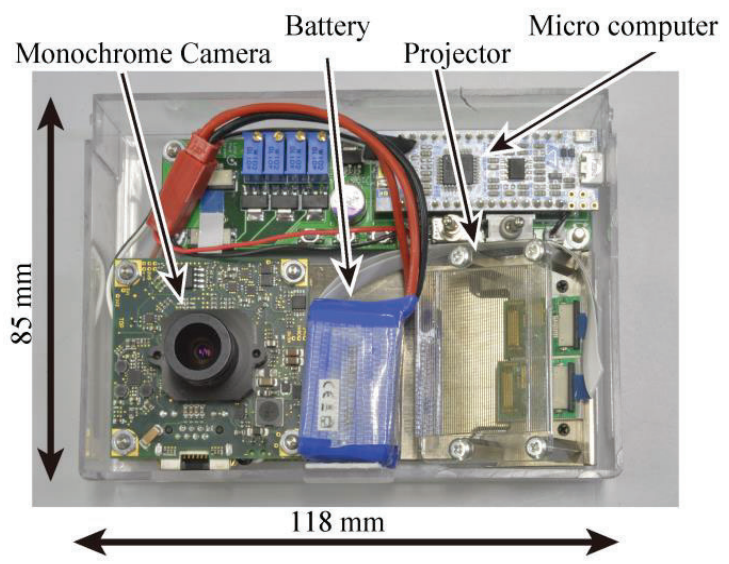

Figure 1. Measurement device.

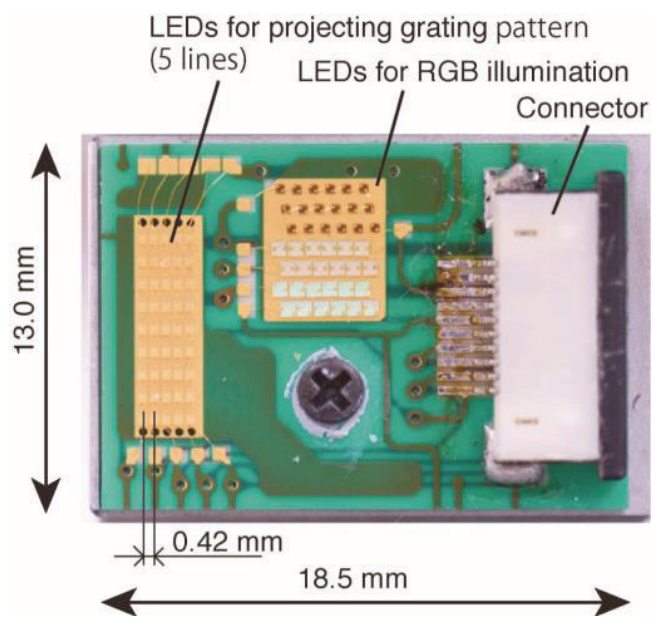

Figure 2. Linear LED device. 


\section{Measurement method}

\section{Whole-Space Tabulation Method (WSTM)}

In this method, the relation table of phase and 3D coordinates is created in advance. Therefore, it is not necessary to perform complicated calculation, and measurement can be performed at high speed.

\section{Method to obtain color information}

Figure 2 shows the linear LED device. RGB LEDs are illuminated for each color and images are grabbed. The luminance values of the three obtained images represent the brightness in the RGB color model. Therefore, color information was obtained by combining three images.

The obtained color information can associate with 3D coordinates because 3D coordinates are obtained for each pixel of the camera.

\section{Real time measurement}

3 steps of grabbing the images, analysis, and display are necessary for 3D measurement. Conventionally, these 3 steps were sequentially performed, and the measurement time was about $720 \mathrm{~ms}$.

Therefore, in order to speeding up, 3 steps are divided into threads and processed in parallel. An outline is shown in Figure 3.

In addition, OpenMP was used to speeding up for the analysis.

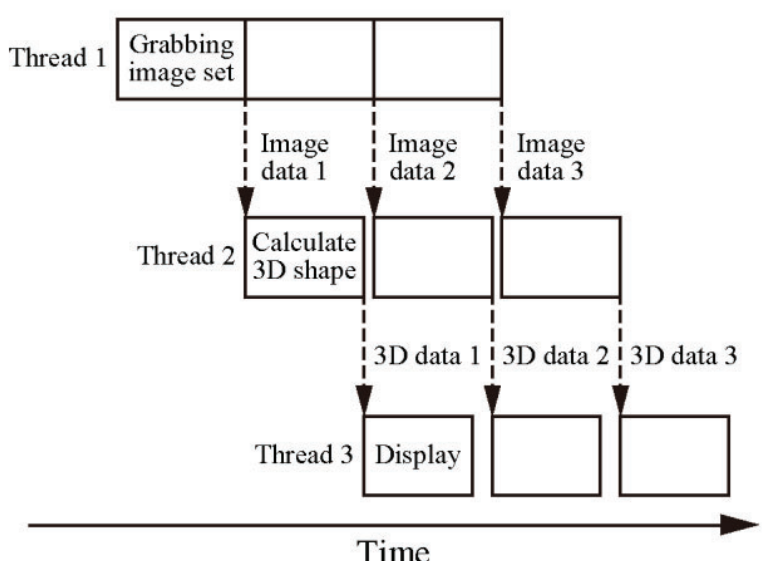

Figure 3. Outline of multithreading processing.

\section{Measurement result}

Figure 4 shows the measurement result. An object is a leopard figure of about $50 \mathrm{~mm}$ in height.

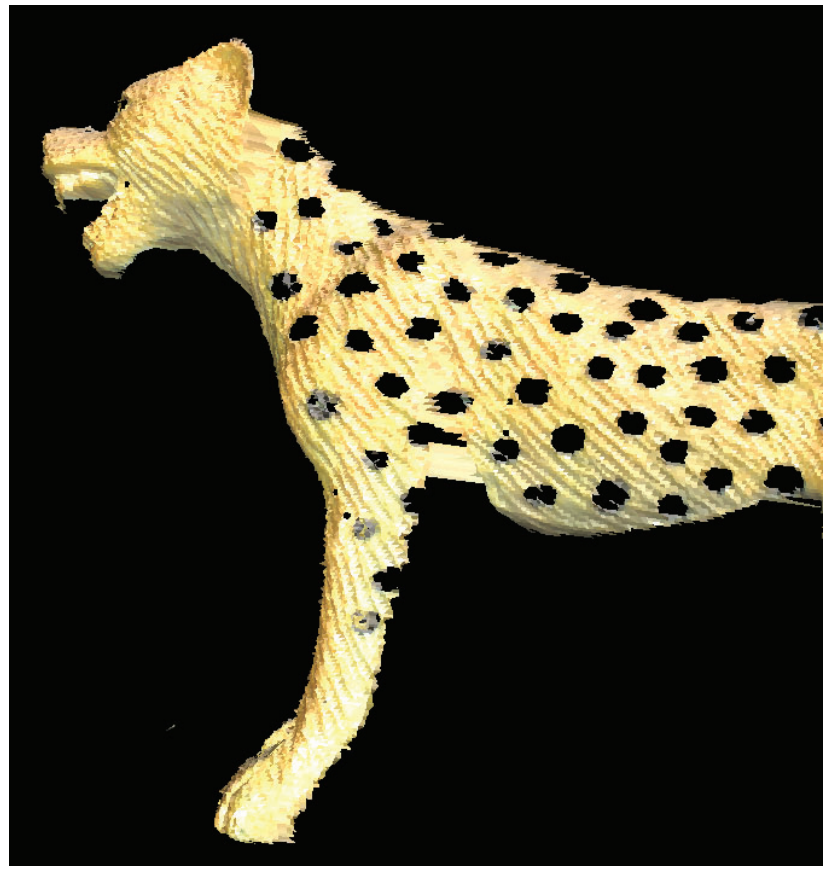

Figure 4. Result of full-color 3D measurement.

\section{Conclusions}

A handy type full color 3D measuring device was prototyped. Also, real-time measurement with full color by multithreading was realized.

\section{References}

[1] M. Fujigaki, Y. Morimoto, "Shape measurement with grating projection using whole-space tabulation method" (in Japanese), Journal of JSEM, 8(4), (2008) 402-408.

[2] M. Fujigaki, T. Sakaguchi, Murata, Y., "Development of a compact 3D shape measurement unit using the lightsource-stepping method", Optics and Lasers in Engineering, 85, (2016) 9-17 


\title{
Accuracy estimation of a 3D reconstruction method for scanning electron microscope images*
}

\author{
Stefan Töberg*a , Eduard Reithmeier ${ }^{\mathrm{a}}$ \\ anstitute of Measurement and Automatic Control, Leibniz Universität Hannover, Nienburger \\ Str. 17, 30167 Hannover, Germany
}

\begin{abstract}
Morphological and microstructural characteristics are often characterized using the images of scanning electron microscopes (SEM). Unfortunately, the acquired image data is limited to two dimensions although the 3D structure of the specimen provides a large amount of classification options. Therefore, 3D reconstruction methods are used to obtain the necessary depth directly from the acquired SEM image data. In this paper, we want to evaluate a $3 \mathrm{D}$ reconstruction scheme, that can be applied to uncalibrated stereo-pair images to compute the depth of the scene directly from the disparity of the rectified stereo-pair, in terms of its accuracy. Registered 3D data acquired from multiple stereo-pairs is presented that allows to evaluate if the obtained results are truly metric reconstructions of the investigated specimen as well as to assess the further application possibilities.
\end{abstract}

Keywords: 3D reconstruction, scanning electron microscope, multiple stereo-pair images, accuracy estimation, evaluation, metric

\section{Introduction}

The scanning electron microscope (SEM) is a reliable tool to characterize the morphological and microstructural characteristics of a large variety of specimen. Its fast nondestructively acquired high-resolution images and wide magnification range make it a versatile measurement system for the micro- and nano-scale. Nevertheless, the obtained image data only contains 2D information. The additional utilization of different 3D measurement systems like for instance atomic force microscopes [1] or confocal laser scanning microscopes [2] is time consuming and not always applicable to the present measurement object. Methods that turn the 2D SEM images into 3D measurements can solve this problem and extend the SEM's fields of application. A possible example is the characterization of particulate matter (PM) that is an air pollutant with impact on the environment and human's health. Most morphological analyses are limited to the evaluation of the 2D image data [3]. The inclusion of the 3D morphology into these analyses can allow to fully characterize several types of PM and find new relevant features. A suitable approach that was presented in [4] enabled us to reconstruct PM agglomerates using multiple SEM stereo-pair images. However, these qualitative results haven't been quantitatively evaluated yet. To truly rely on the

\footnotetext{
*Toeberg@imr.uni-hannover.de; phone: +49 511 762-4284
}

reconstructed data an estimation of the result's accuracy is inevitable. Therefore, a method is presented to calibrate the scale of the acquired stereo-pair images precisely using a multiranged calibration pattern in form of a gold-silicon checkerboard. The corners are accurately detected by a robust saddle point refinement that is suited for noisy SEM images. With the exact scale the $3 \mathrm{D}$ reconstruction method can be evaluated regarding its accuracy using a sphere with known dimensions. Furthermore, the $3 \mathrm{D}$ reconstruction procedure is optimized and the assumptions it is based on are analysed. The possible error sources as well as limiting factors are outlined.

\section{Methods}

\section{Calibration}

The used calibration pattern consists of a fourfold gold-silicon checkerboard pattern. To determine the scale of the acquired images, the checkerboard's corners have to be detected. This is done by initially defining a region of interest (ROI) in that a corner detection is processed using the Shi-Tomasi corner detector [5]. The coordinates of this corner are used to define a new ROI for the saddle point refinement. First the defined image section is interpreted as a height map, so that the greyscale values become depth values. Subsequently a plane with a polynomial of degree six is fitted to these depth values using linear least-squares. The gradients of this plane are computed in $\mathrm{x}$ - and $\mathrm{y}$-directions at defined subpixel grid points. 


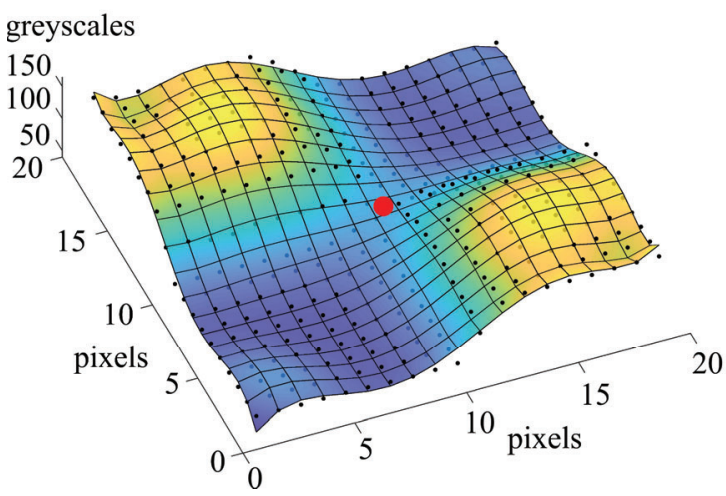

Figure 1. Plane of degree six fitted to the greyscale values of the pixels (black). The red point marks the coordinates of the detected saddle point.

Finally, the position of the minimal gradient sum is identified representing the coordinates of the refined checkerboard corner with subpixel accuracy illustrated as a red point in Fig. 1. An additional checkup if the detected extremum is really the saddle point and not a minimum or maximum is not necessary if the size of the ROI is chosen correctly. Furthermore, the sole use of the first gradients without an additional checkup can also be applied to other structures on the checkerboard like arrows marking the distances between two neighboured checkerboards. By using the saddle point refinement, the correction of the initially detected corners can vary between several pixels and subpixels like demonstrated in Fig. 2. Between the coordinates of the detected corners the Euclidean pixel distance is calculated and assigned to the metric distance of the known checkerboard dimensions. If the scanning unit of the SEM is properly adjusted, the correspondence between the pixel length and metric length for the $\mathrm{x}$ - and $\mathrm{y}$-axis are equal. In addition, the effects of distortions in images, captured by modern SEM imaging systems, are neglectable and don't have to be considered [6].
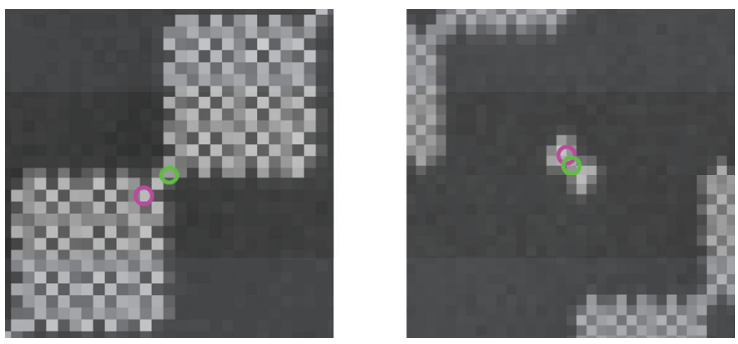

Figure 2. Detected corners by the Shi-Tomasi corner detector (magenta) and refined corners (green) generated by the extreme/saddle point detection.

\section{3D reconstruction}

To acquire the necessary stereo-pair images, the equivalence between object and camera motion is exploited. That means the investigated specimen is tilted in a way that minimizes the change of the imaged scene. By adjusting the scan rotation and working distance, the focus of the scene can be practically maintained and the movement of image points is almost exclusively reduced to the horizontal image axis during the tilting.

The stereo-pair images are then used to robustly estimate the fundamental matrix from automatically detected and matched point correspondences. Good results were achieved using KAZE feature points [7] and SIFT descriptors [8] as well as the normalized 8-point algorithm [9] embedded in a robust RANSAC [10] scheme with an optional subsequent non-linear optimization of the fundamental matrix. The determined epipolar geometry or rather the obtained outlier-free point correspondences allow to rectify the stereo-pair images, reducing the dense correspondence problem between the two stereo-pair images along conjugate epipolar lines that correspond to the rows of the rectified images like illustrated in Fig. 3. To rectify the images with no further knowledge about the intrinsic parameters of the imaging system, the quasi-Euclidean epipolar rectification method [11] is used. The algorithm aims to approximate the "ideal" Euclidean epipolar rectification [12] by forcing the rectyfing transformations to have the same structure as in the calibrated case, collineations induced by the plane at infinity. This results in mostly distortion-free rectified images. To control the occurring amount of distortions in the transformed images, the results are only accepted if the obtained images' corner angle values vary up to $\pm 1^{\circ}$ from a right angle. Otherwise another set of point correspondences with a similar deviation to the related fundamental matrix is chosen and the procedure is repeated.

The rectification of the images enables to densely match the pixels of the stereo-pair obtaining the horizontal pixel displacements. To compensate possible radiometric differences and offsets in the images' intensities, a rank transform is used [13]. It is well suited to improve the dense matching process of the SEM images regarding its robustness and allows to yield results with less mismatches. The rank transform replaces the intensity of a pixel by the number of pixels with a higher intensity than the center pixel in a window with chosen size [14]. After this transform an initial disparity is determined using the sampling-insensitive absolute difference according to Birchfield and Tomasi [15] that computes the absolute distance between the 


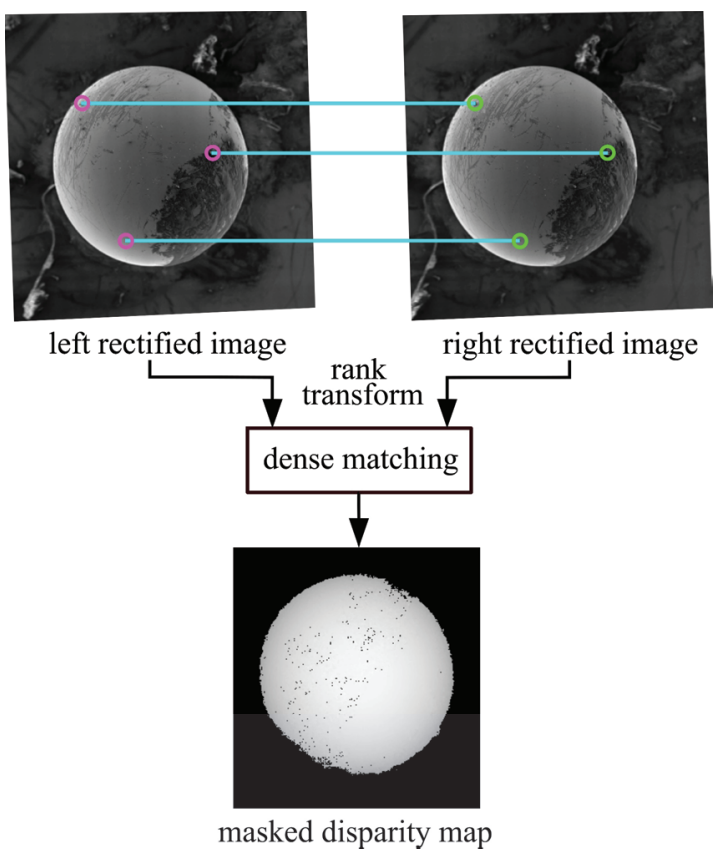

Figure 3. Corresponding points in the rectified images lying in the same pixel row. After the rectification, the images are rank transformed and densely matched. The obtained disparity map (disparity values displayed in greyscales) is masked (invalid pixels in black) and subsequently used for the depth computation.

extrema of linear interpolations of the corresponding pixels of interest with their neighbors. Additionally, a cost function is used to enforce a global smoothness constraint of the disparity map that is approximated by combining eight symmetrical, onedimensional pathwise optimizations for each pixel [16]. Finally, for each pixel, the disparity with the lowest cost is chosen.

As the last step of the reconstruction process, the relative depth $h$ can be computed using the triangles illustrated in Fig. 4. according to [17]. Assuming the tilt angle $\varphi$ is known, the depth can be defined by the tilt angle and the distances between the eucentric point $E$ and the projections $P$ and $P^{\prime}$ of the corresponding image points on a reference plane in the form of

$$
h=\frac{E P \cos (\varphi)-E P^{\prime}}{\sin (\varphi)} .
$$

Using a small-angle approximation, assuming $\cos (\varphi) \approx 1$ and $\sin (\varphi) \approx 2 \sin (\varphi / 2)$, the depth $h$ can directly be calculated from the disparity $d$ of the rectified images via

$$
h=\frac{d \cdot p}{2 \sin (\varphi / 2)} .
$$

$p$ stands for the scale of the images, transforming the pixel values into metric units. Formula (2) is valid because the imaging system of the SEM can be described by the parallel projection model. The incident electron beam impinges nearly rectangular on every point of the specimen's surface that is part of the raster scan pattern (Fig. 4). This approximation is accurate because the working distance and focal length of the virtual imaging system is significantly larger than the investigated specimen. This approximation gets more precise the higher the used magnification is.

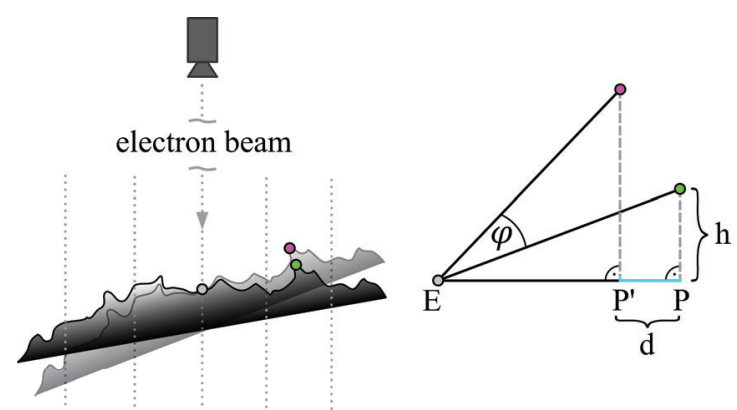

Figure 4. Illustration of the virtual imaging system of the SEM as well as the triangles that result from the stage tilt with the tilt angle $\varphi$, the computed depth $h$ and the disparity $d$.

\section{Registration}

The individual reconstructions of multiple stereo-pair images can be registered and merged to reconstruct occluded areas as well as enlarge the measurement area. To register the reconstructions of two different stereo-pairs, feature points in the left images (the images, the disparity value is counted from) of each individual stereo-pair are detected and matched. These point coordinates are assigned to the computed depth values obtaining two corresponding 3D point sets. These 3D point sets can be matched using a suitable transformation that has to be able to compensate two different sources of occurring deviations. First, it has to compensate the coordinate change of the points in the image plane that is caused by the tilting and the resulting different perspectives as well as the weak distortions of the rectifying transformations that are slightly different for each image and stereo-pair. Second, if the actual tilt angle doesn't equal the estimated one, for instance caused by inaccuracies of 
the stage mechanics, the depth has to be adjusted to the fixed point cloud that functions as a reference. An affine transformation is capable of compensating both sources of deviations appropriately. It is demonstrated that the actual reconstruction results are not falsified.

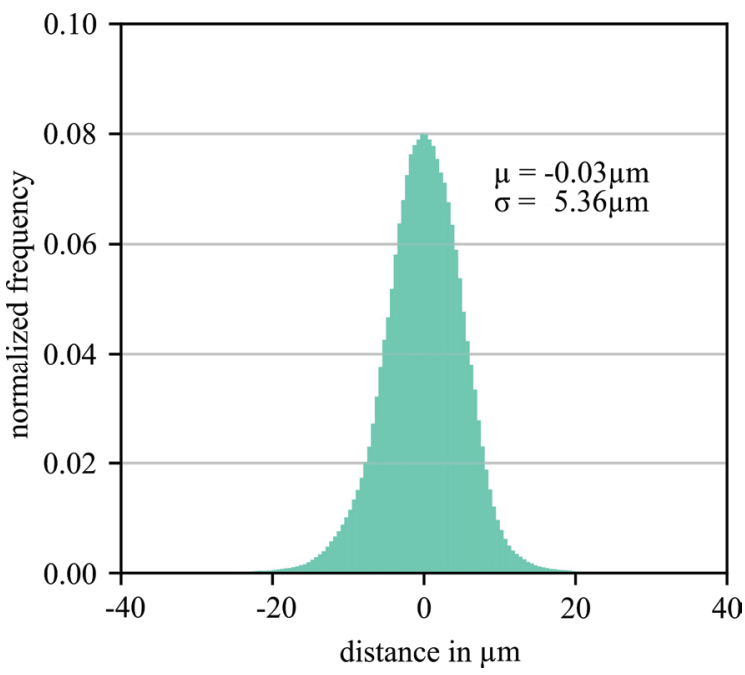

Figure 5. Histogram of the obtained cloud to cloud distances using the least-squares fitted sphere with a radius of 498,2 $\mu \mathrm{m}$ as the reference. (bin width $0,5 \mu \mathrm{m}$, total vertices $\sim 3.5$ million)

\section{Results and Discussion}

The accuracy of the outlined approach is evaluated reconstructing a sphere with a known diameter. Three stereopairs were used with following tilt angles: $-15^{\circ} /-10^{\circ}, 5^{\circ} / 10^{\circ}$ and $25^{\circ} / 30^{\circ}$. For the registration the images of $30^{\circ}$ and $10^{\circ}$ as well as $10^{\circ}$ and $-10^{\circ}$ were used to determine the matching affine transformations between the three clouds. The reconstructed point cloud of the stereo-pair $-15^{\circ} /-10^{\circ}$ was used as the fixed point set for the registration process. For the approximately 3.5 million reconstructed vertices, the Euclidean distance to a leastsquares fitted sphere, functioning as the ground truth, was calculated using vector algebra. The radius of the fitted sphere is $498,2 \mu \mathrm{m}$ what equals the calibration sphere's metric radius of $500 \pm 50 \mu \mathrm{m}$ as specified by the manufacturer. In Fig. 5 the histogram of the obtained distances is illustrated. It becomes clear that the reconstruction noise can be considered as normally distributed and is probably directly caused by matching deviations that can be attributed to various factors like the overall image quality, the noise that is present in the images and the changes between the stereo-pair images, for instance caused by the variation of focus or lightning. That means the standard deviation $\sigma$ is as well scaled with the constant $p$ converting pixels into metric units. Therefore, it should be equal on a percentage basis for higher magnifications assuming the image quality or rather the image noise level is comparable. So far, this could be confirmed by the obtained reconstruction results for higher magnifications. In Fig. 6, the finally created mesh and its deviations from the least-squares fit are illustrated. It was created using a Poisson surface reconstruction that handles noise very well and slightly reduces the overall error [18].

The presented results demonstrate that the obtained reconstructions can be considered as metric measurements as well as that the parallel projection model is also applicable to SEM images acquired at a lower magnification producing good results in terms of accuracy. Although the point clouds were registered using affine transformations that were computed with point sets, only representing small parts of the whole point clouds, the shape of the sphere has been maintained and no remarkable loss of accuracy can be noticed in the registered parts. The minimum and maximum angles describing the reconstructed parts of the sphere can be vectorially calculated starting at about $-75^{\circ}$ up to $115^{\circ}$ considering the maximum point of the sphere in the illustrated position as $0^{\circ}$. High tilt angles are necessary to provide a sufficient resolution of steep flanks that can't be reconstructed well otherwise.

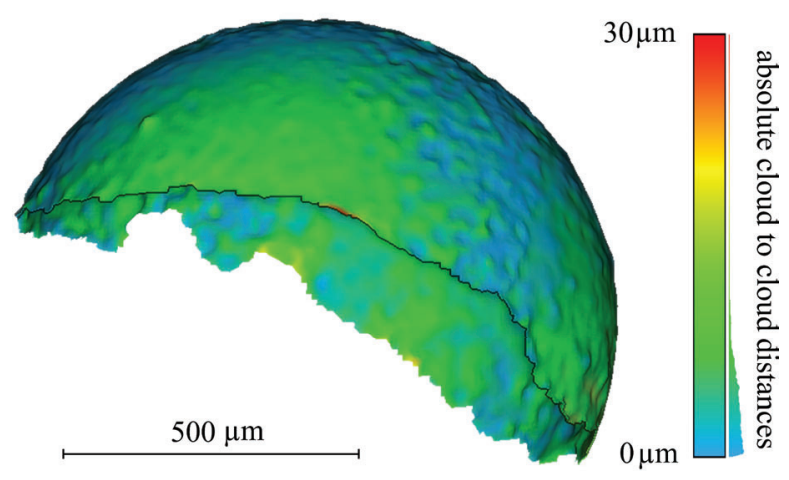

Figure 6. Absolute cloud to cloud distances between the vertices of the finally computed mesh and the leastsquares sphere fit of the reconstructed point cloud.

To conclude, the presented method enables to metrically reconstruct specimen in the SEM. It is important that the overall quality of the acquired images is high because blurred and noisy images impair the dense matching strongly. Faulty values and noise in the disparity reproduce directly in the reconstructions 
and falsify the obtained 3D data. In addition, the precision of the microscope's stage is very important because a wrongly estimated angle causes a relative error directly proportional to the ratio of the true and estimated angle. Thus, to use the presented approach in the nanometer range, it is recommended to install an ultra-precision stage in the used SEM.

\section{References}

[1] Leach, R. and Artigas, R., [Optical measurement of surface topography]. Springer, Berlin \& Heidelberg, 237-286 (2011).

[2] Morita, S., Giessibl, F. J., Meyer, E., and Wiesendanger, R., [Noncontact atomic force microscopy], Springer, (2015).

[3] Liati, A., Eggenschwiler, P. D., Gubler, E. M., Schreiber, D., and Aguirre, M., "Investigation of diesel ash particulate matter: a scanning electron microscope and transmission electron microscope study," Atmospheric environment, 49, 391-402 (2012).

[4] Töberg, S., and Reithmeier, E., "3D reconstruction of particle agglomerates using multiple scanning electron microscope stereo-pair images," International Society for Optics and Photonics, Optical Metrology and Inspection for Industrial Applications V 10819, 108190I (2018).

[5] Shi, J., and Tomasi. C., "Good features to track," Cornell University, (1993).

[6] Cui, L., and Marchand, E., "Calibration of scanning electron microscope using a multi-image non-linear minimization process," Robotics and Automation (ICRA), IEEE International Conference, 5191-5196 (2014).

[7] Codevilla, F., Gaya, J. D. O., Duarte, N. and Botelho, S., "Achieving turbidity robustness on underwater images local feature detection," International journal of computer vision 60(2), 91-110 (2004).

[8] Lowe, D. G., "Object recognition from local scale-invariant features," Seventh international conference on computer vision, Proc. IEEE 2, 1150-1157 (1999).

[9] Hartley, R. and Zisserman, A., [Multiple view geometry in computer vision]. Cambridge university press, (2003).

[10] Fischler, M. A. and Bolles, R. C., "Random sample consensus: a paradigm for model fitting with applications to image analysis and automated cartography," Communications of the ACM 24(6), 381-395 (1981).

[11] Fusiello, A., and Irsara, L., "Quasi-euclidean uncalibrated epipolar rectification," 19th International Conference on Pattern Recognition, IEEE, 1-4 (2008).
[12] Fusiello, A., Trucco, E. and Verri, A., "A compact algorithm for rectification of stereo pairs," Machine Vision and Applications 12(1), 16-22 (2000).

[13] Scharstein, D. and Szeliski, R., "A taxonomy and evaluation of dense two-frame stereo correspondence algorithms," International journal of computer vision 47(1-3), 7-42 (2002).

[14] Zabih, R. and Woodfill, J., "Non-parametric local transforms for computing visual correspondence," European conference on computer vision, 151-158 (1994).

[15] Birchfield, S. and Tomasi, C., "A pixel dissimilarity measure that is insensitive to image sampling," IEEE Transactions on Pattern Analysis and Machine Intelligence 20(4), 401-406 (1998).

[16] Hirschmüller, H., "Stereo processing by semiglobal matching and mutual information," IEEE Transactions on pattern analysis and machine intelligence 30(2), 328-341 (2008).

[17] Xie, J., "Stereomicroscopy: 3D imaging and the third dimension measurement," Application Note, Agilent Technologies (2011).

[18] Kazhdan, M., and Hoppe H., "Screened poisson surface reconstruction," ACM Transactions on Graphics 32(3), 29 (2013). 


\title{
Design of FPGA-based signal-processing system based on the direct phase determination method for heterodyne interferometry
}

\author{
Thanh Dong NGUYEN, Quang Anh DUONG, Masato HIGUCHI, Dong WEI, and Masato \\ AKETAGAWA*
}

\author{
Department of Mechanical Engineering, Nagaoka University of Technology, 1603-1, Kamitomioka \\ Nagaoka, Niigata, 940-2188, Japan
}

\begin{abstract}
A signal-processing system based on the direct phase determination method [1] for high-speed phase measurements in real time is presented with the use of a field programmable gate array (FPGA) device. The system running at a clock rate of $120 \mathrm{MHz}$ is designed under LabVIEW environment. The system is investigated using two $2.7 \mathrm{MHz}$-sinusoidal signals from a function generator, one of which is phase-modulated. The result shows that the system can achieve the phase measurement with a modulation frequency up to $5 \mathrm{kHz}$.
\end{abstract}

Keywords: phase-locked loop, null condition, heterodyne interferometer, FPGA, signal-processing

\section{Introduction}

Recently, the signal-processing system based on the direct phase determination method has been developed for high-speed phase measurements in heterodyne interferometry [1]. However, the system is not executed in real time, and it is, therefore, not conventional for the system to apply for industrial applications.

In this paper, we develop another FPGA-based signalprocessing system for high-speed phase measurements in real time. The system provides the first experimental result with a phase-modulation frequency of $5 \mathrm{kHz}$.

\section{Signal-processing system}

\section{System architecture}

The principle of the direct phase determination has been presented in [1]. Figure 1 shows the signalprocessing architecture of the method. Two $2.7 \mathrm{MHz}$ sinusoidal signals (reference and measurement) from a function generator are sampled by an available ADC (analog-to-digital) device at $120 \mathrm{MHz}$. For most heterodyne interferometers, the displacement of a target alternates the measurement phase. This means that the difference in phase between the two inputs signals is varied by the displacement when the reference phase is stationary. The main program with the use of the phaselocked loop (PLL) and null method runs on a FPGA module at $120 \mathrm{MHz}$. The host program stores data into a storage file in real time. Because the phase shift is detected at ultra-high frequency $(120 \mathrm{MHz})$, the artificial periodic error appeared needs to be removed by postprocessing (figure 1(c)).

The main FPGA program consists of a phase detector (multiplier), a low-pass filter (LPF), an integrator, and a phase shifter. It assumes that the reference $\left(I_{l}\right)$ and the measurement $\left(I_{2}\right)$ are given by

$I_{1}=A \cos \left(\Delta \omega t-\frac{\pi}{2}\right)=A \sin (\Delta \omega t)$,

$I_{2}=B \cos \left(\Delta \omega t+\theta_{m}\right)$,

where $A$ with $B, \Delta \omega$ and $\theta_{m}$ are amplitudes of the $I_{1}$ and $I_{2}$ signals, the angular beat frequency, and the unknown phase of the $I_{l}$ due to the displacement, respectively.

\section{Phase shifter}

The phase shifter drifts an any real $\Phi$ phase of the reference signal so that it satisfies the null condition (the $\Phi$ phase is controlled to be the $\theta_{m}$ phase). From figure 1(a), the $\Phi$-shifted reference signal is described as $I_{1}^{\prime}=A \sin (\Delta \omega t+\Phi)$.

To create the $I_{1}{ }_{1}$ signal, the in-signal element and the quadrature element generated by a Hilbert transformer are multiplied by trigonometric functions of the $\Phi$ phase.

\section{Phase detector (multiplier)}

*masatoaa@vos.nagaokaut.ac.jp; phone 0258-47-9741 
It provides a product of the $I^{\prime}{ }_{1}$ and $I_{2}$, described as

$$
I_{1}^{\prime} \times I_{2}=\frac{1}{2} A B\left[\sin \left(2 \Delta \omega t+\Phi+\theta_{m}\right)+\sin \left(\Phi-\theta_{m}\right)\right] \text {. }
$$

The product contains a subtraction of $\Phi-\theta_{m}$, which is related to the displacement. The FPGA program allows the $\theta_{m}$ to be locked by applying the PLL and null method.
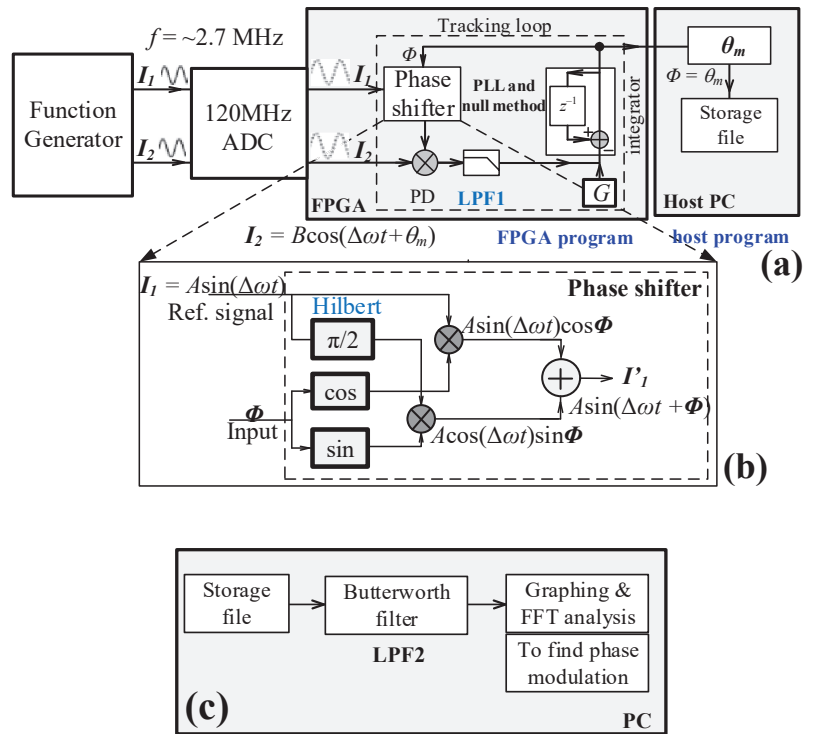

Figure 1. Scheme of the signal-processing performance: (a) an overall view of system, (b) phase shifter, (c) post-processing.

\section{Hilbert transformer and low pass filter}

Both the Hilbert transformer and the LPF are designed as high-throughput $N$-tap FIR (finite impulse response) filters that run at the FPGA clock rate of $120 \mathrm{MHz}$. The FIR filter is expressed as

$$
y(k)=\sum_{n=0}^{N-1} h(n-1) x(k-n),
$$

where $h[k]$ and $x[k]$ are the filter coefficient and the input data, respectively.

To build FIR filters on the FPGA, FIR coefficients with their typical characteristics are firstly acquired by the Digital Design Toolkit [2]. The Hilbert transformer aims to generate the quadrature element $\left(I_{l}\left(90^{\circ}\right)=A \cos (\Delta \omega t)\right)$ whose phase is shifted by $90^{\circ}$ with respect to that of the in-signal $\left(I_{I}\left(0^{0}\right)=A \sin (\Delta \omega t)\right)$. Meanwhile, the role of the LPF (LPF1) is to remove the high-frequency term of the $I^{\prime}{ }_{1} \times I_{2}$ multiplication. Therefore, we select a 32-tap FIR filter as the Hilbert transform that has a frequency range of $[0.05,0.45]$ with an amplitude of $[-1,-1]$ established in

a normalized frequency range of $[0,0.5]$. A 42-tap Hann filter with a ratio $f_{c} / f_{s}=1.8 \mathrm{MHz} / 120 \mathrm{MHz}=0.015 \mathrm{~Hz} / 1 \mathrm{~Hz}$ ( $f_{c}$ : cuttoff frequency, $f_{s}$ : sampling rate) is selected in the $[0,0.5]$ in the case that the modulation frequency of the $I_{2}$ signal is in $[500 \mathrm{~Hz}, 10 \mathrm{kHz}]$. Magnitude and phase responses of the 32-tap Hilbert filter and the 42-tap LPF are shown in figure $2 \& 3$, respectively.

An IP Generator tool is an interactive way for users to induce high-throughput FIR filters on the FPGA [3]. With using this tool, two design types of the FIR filter including Multiply-Accumulate (MAC) [4] and Distributed Arithmetic (DA) [5] are used on FPGA platforms. To balance between the processing velocity of the filters at $120 \mathrm{MHz}$ and hardware-resource usage, we select the DA structure, and the single-rate type where all functions run in a Single-Cycle Timed Loop (SCTL) at one FPGA clock tick [6].

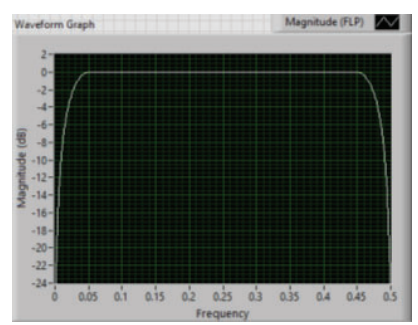

Figure 2. Characteristics of 32-tap Hilbert transformer in $[0-0.5 \mathrm{~Hz}]$ : (a) magnitude, (b) phase
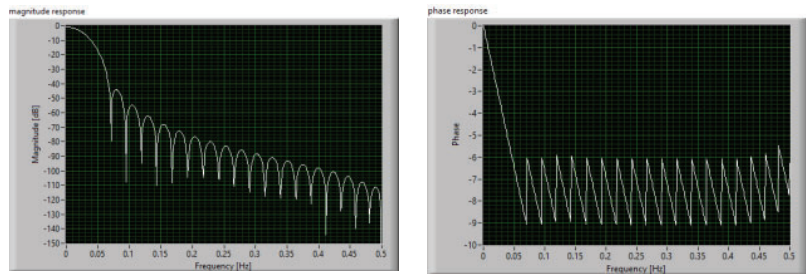

Figure 3. Characteristics of 42-tap filter (LPF) in [0- $0.5 \mathrm{~Hz}]$ : (a) magnitude, (b) phase.

\section{Gain coefficient}

The user can adjust a $G$ loop gain via the measurement signal to adapt the PLL time response as well as the locking stability. The $G$ selection varies if the measurement is phase-modulated at different frequencies.

\section{Integrator}

To collect the phase shift, we choose a first-order integrator (figure 1). The transfer function $(F(z))$ of the integrator in the $z$-transform domain is written by

$F(z)=\frac{y[k]}{x[k]}=\frac{-1}{1-z^{-1}}$. 
where $x[k]$ and $y[k]$ are the $k$ th-order input and output (phase shift) of the integrator, respectively.

\section{Interconnection between sub-parts using FIFOs} (first-in-first-out buffers) memories in FPGA program

Figure 4 shows an overall view of the signal-processing performance of the FPGA program. Three SCTLs consists of data acquisition, Hilbert transformer processing and main processing (PLL and null method). All loops run at the same ADC frequency of $120 \mathrm{MHz}$ on the FPGA and are implemented continuously in a parallel working mode. There are two frequency types that include $120 \mathrm{MHz}$ (or $480 \mathrm{MHz}$ ) and 4 cycles/ sample in the program. $120 \mathrm{MHz}$ (or $480 \mathrm{MHz}$ ) are FPGA clock rates, and 4 cycles/ sample express the throughput $(h)$ of the filter that indicates the minimum input interval between two adjacent inputs. For our available FPGA (NI 7279R), the default FPGA clock rate is $40 \mathrm{MHz}$. However, the FPGA uses a PLL technique to create multiples of $40 \mathrm{MHz}(80 \mathrm{MHz}, \ldots \sim \mathrm{GHz})$ [7]. The actual throughput $(H)$ in MSample/ second (Ms/s) of the FIR filter is given by

$$
H=\frac{F P G A-\text { clock }- \text { rate }}{h}=\frac{M H z(s / \text { cycles })}{\text { cycles } / \text { sample }}=M s / s
$$

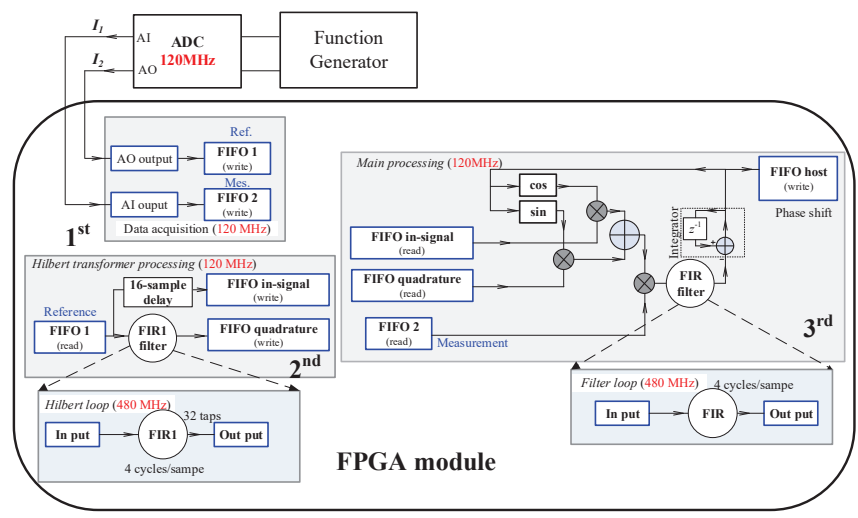

Figure 4. An overall view of signal-processing performance (FPGA program) on the FPGA module.

The user has the possibility to choose other FPGA clock rates and other $h$ throughputs to make sure the $H$ throughput of two filters (FIR1 and FIR) running at 120 MHz. In the data acquisition loop $\left(1^{\mathrm{st}}\right)$, the $I_{1}$ and $I_{2}$ sequences are acquired at $120 \mathrm{MHz}$. In the Hilbert transformer processing loop $\left(2^{\text {nd }}\right)$, the reference sequences are read from the FIFO1 memory. The reference is filtered by the 32-tap Hilbert transformer (FIR1) to generate the quadrature. The reference is also delayed by 16 samples to create the in-signal. In the main processing loop $\left(3^{\text {rd }}\right)$, the phase shift generated is stored into the FIFO host memory. The FIFO host finally transfers the data to the host computer.

\section{Experiments and Results}

To validate the signal-processing performance, two

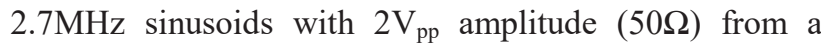
function generator (Agilent 33522A) are provided to the method. The main program is compiled on the FPGA (NI7279R) equipped with the ADC (NI5733, 120MHz sampling rate). The $I_{2}$ measurement signal is phasemodulated at $5 \mathrm{kHz}$ with a sinusoidal waveform and a deviation of $\pm 360^{\circ}$. The data is stored into the storage file in real time. The post-processing program utilizing a Butterworth filter $\left(\mathrm{LPF} 2\right.$, order $=2, f_{c} / f_{s}=15 \mathrm{kHz} / 120$ $\mathrm{MHz}$ ) is necessary to remove the high-frequency artificial error. In our experiment, we select $G=0.3$ (fixed-point, $< \pm 16,1>$ ). The experimental configuration is setup as figure 1. Figure 5(a) shows the phase response of the phase shift in the domain time and a fast Fourier transform (FFT) analysis displays its frequency spectrum as shown in figure 5(b). The highest frequency peak is $\sim 5$ $\mathrm{kHz}$, and a peak-to-peak amplitude of $\sim 720^{\circ}$ is obtained.
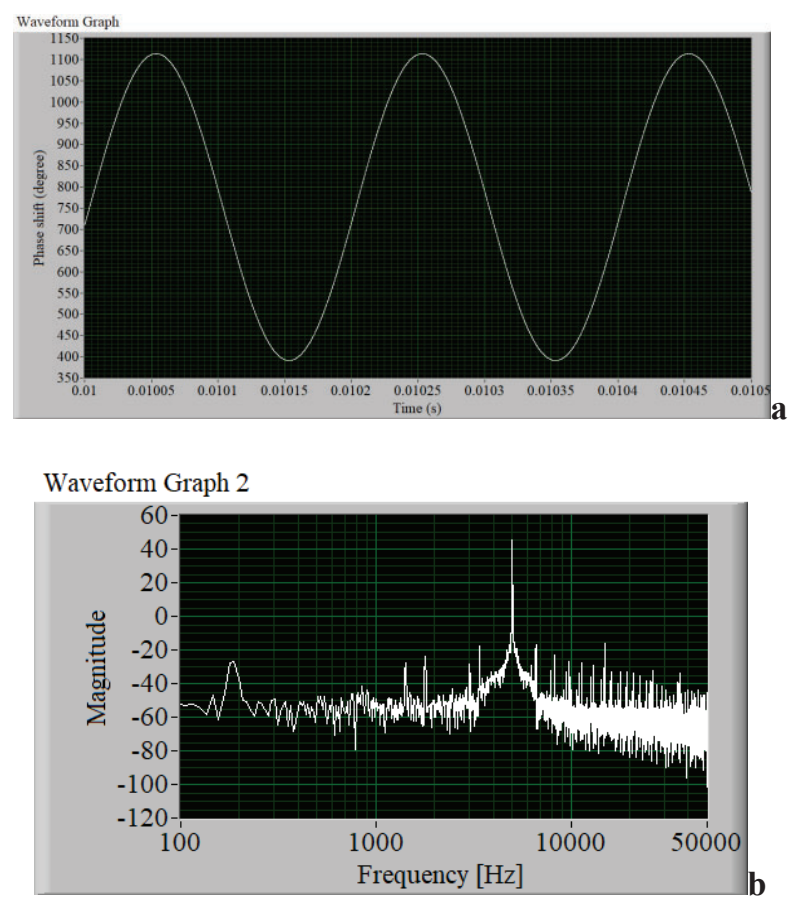

Figure 5. The measurement result: (a) phase change in the phase shift, (b) fast Fourier transform (FFT) analysis of figure 5(a); the highest peak is $\sim 5 \mathrm{kHz}$. 


\section{Conclusion}

In this paper, we have designed the real-time signalprocessing system based on the direct phase determination method. The system provides the highspeed phase measurement with a modulation frequency up to $5 \mathrm{kHz}$ using two $2.7 \mathrm{MHz}$ sinusoidal signals. The system is capable of being used for high-speed phase measurements in industrial applications. In the future, with improving this system, we will (1) extend the phase measurement with modulation frequencies up to tens of $\mathrm{kHz}$, and more than; (2) investigate the measurement resolution for heterodyne interferometers.

\section{References}

[1] Dong, N. T, Anh, D. Q., Higuchi, M., Wei, D., Aketagawa, M., "Direct phase determination using phase lock loop for heterodyne displacement-measuring interferometers", Proc. Photonic Asia Conference, 2018.

[2] National Instruments, "LabVIEW Digital Design Toolkit User Manual", February 2005, < http://www.ni.com/pdf/manuals/371353a.pdf

[3] National Instruments, "Designing Ultra-High Throughput FIR Filters with no Multiplier on NI FPGA Platforms", 23 August 2013, <http://www.ni.com/whitepaper/9700/en/>

[4] Anees, N. J. M., Kharate, A. B., "FPGA based implementation of MAC in Parallel Filter", International Journal of Innovation Research in Science Engineering and Technology, 18460-18467 (2017).

[5] Keerthi, M., Midasala, V., Bhavanam, S. N., Jeevan, K. R., "FPGA Implementation of Distributed Arithmetic for FIR filter", International Journal of Innovation Research in Science Engineering and Technology 1(9) (2012).

[6] National Instruments, "Single-Cycle Timed Loop FAQ for the LabVIEW FPGA Module", 25 July 2018, < https://knowledge.ni.com/KnowledgeArticleDetails?id=k A00Z000000P8sWSAS\&l=en-CA >

[7] National Instruments, "NI 5731/5732/5733/5734R User Guide and Specifications", February 2011, < http://www.ni.com/pdf/manuals/375653a.pdfs 


\title{
High-speed ghost imaging by deep learning*
}

\author{
Yasuhiro Mizutani*, Otoki Yagi, Yasuhiro Takaya \\ Department of Mechanical Engineering, Osaka University, 2-1 Yamadaoka, Suita, Osaka 565-0879, \\ Japan
}

\begin{abstract}
An improvement of imaging time for the ghost imaging is realized by using deep learning. The Ghost imaging (GI) is one of a single pixel imaging by correlation between illumination patterns and detected light intensities. The GI has an advantage for weak light intensity. However, it is difficult to obtain a fast phenomenon because of many illuminations for correlation analysis. To overcome the problem, we have applied a deep learning technique for reducing numbers of measurement. In the matter of a deep learning, the proposed method deals with a convolutional neural network. As a result, we have developed 60 times faster than the conventional GI. Additionally, we have observed a moving microparticle with $0.08 \mathrm{sec}$.
\end{abstract}

Keywords: ghost imaging, single pixel imaging, deep learning, convolutional neural network

\section{Introduction}

The Ghost imaging (GI) is one of single pixel imaging methods and a correlation-based technique between intensity distributions of an illumination light and light intensities detected by a bucket detector [1,2]. Because of its simple setup, the GI is widely used in the field of quantum optics, medical imaging, optical astronomy and industrial inspection. Additionally, the GI is also attractive from the point of view of an imaging for weak light intensity. However, it is very difficult to obtain images for a moving sample due to many measurements for acquiring clear images for correlation-calculations. Therefore, the GI have been confined to applying for statically objects. To overcome the problem, we focused on a deep learning (DL) for reducing the GI measurements. DL is applied for prediction of true values by using lower information. Specifically, for imaging field, DL can reconstruct high resolution images by using lower resolution images. While, an image quality of the GI with lower measurements is low even though an information of a sample is including in the GI image. Accordingly, for recover the information, we attempt to apply the DL to the GI with lower measurements. As a result, it was successful to develop the GI with 60 times faster than the conventional GI. Additionally, we have observed a moving micro-particle with time resolution of $0.08 \mathrm{sec}$.

*mizutani@mech.eng.osaka-u.ac.jp; phone 81668797319

\section{Principle of the fast GI with deep learning}

Figure 1 shows a schematic illustration of a concept of a fast GI with DL. Random patterned illuminations are irradiated onto a sample. Then intensities of interactive light between the light and the sample, such as scattering, transmittance, fluorescence and so on, is detected by a bucket detector. A distribution of correlation efficiencies $G(x, y)$ is written as

$$
G(x, y)=<\Delta I(x, y) \Delta B_{n}>
$$

where $\Delta I(x, y)$ is a fluctuation of the distribution of the illuminated light, $\Delta B_{\mathrm{n}}$ is a fluctuation of an intensity of a $\mathrm{n}$-th detected light and $\diamond$ is ensemble average. A quality of an image depends on the measurement times. For obtaining a clear image of the GI, it is necessary to measure too many times.

To overcome the problem of the measurement times, we have adopted the convolutional neural network $(\mathrm{CNN})$ as used in the field of image processing. In this work, we derived a possibility of light in each pixel. Figure 2 shows a block diagram of the $\mathrm{CNN}$ as used in this work. The CNN involved some layers about a convolution and activation function. Furthermore, we repeated the set at three times. Finally, the probability map has been derived from the CNN. 


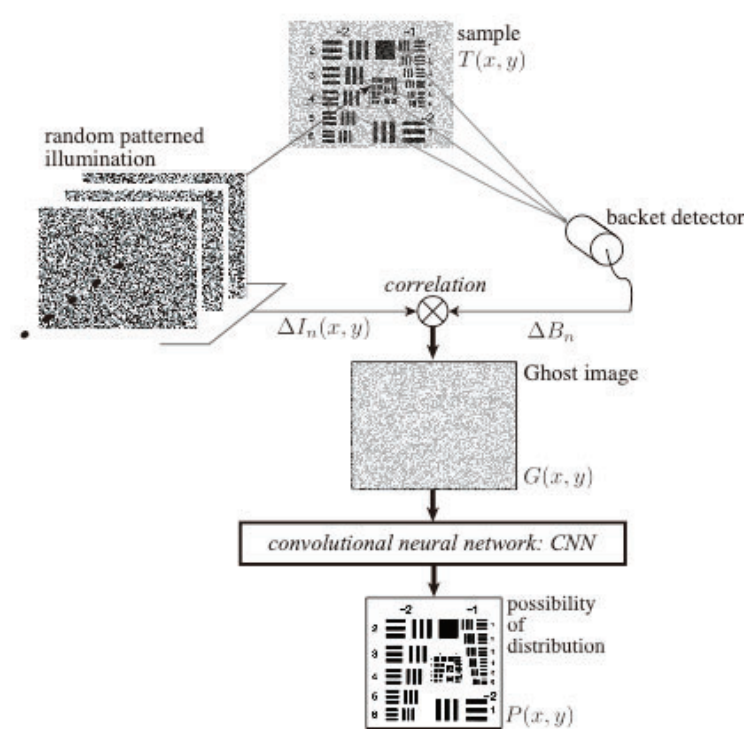

Fig. 1 Schematic illustration of concept for fast ghost imaging with deep learning.

\section{Numerical and experimental results}

Figure 3 shows numerical analysis for the GI with DL. We set a particle like sample as shown in fig. 3(a). In this analysis, we have used 5 times measurement. As shown in fig. 3 (b), the general GI could not reconstruct the particle image. On the other hand, as shown in fig. 3 (c) and (d), the particle could be detected clearly.

Figure 4 shows experimental results for detecting a moving particle by the GI with DL and 5 times measurements. The sample was 5 um glass beads and we have used our microscopic GI system [3]. As shown in fig. 4 (a), the image was captured in each 0.08 sec. Fig. 4 (b) shows a time dependent behavior of the moving particle. We have succeeded to develop the high-speed GI with DL.

\section{Conclusions}

This paper describes the high-speed GI with DL. We have combined the general GI with the CNN which derives a light probability distribution. We have succeeded to capture images of a moving particle in each $0.08 \mathrm{sec}$.
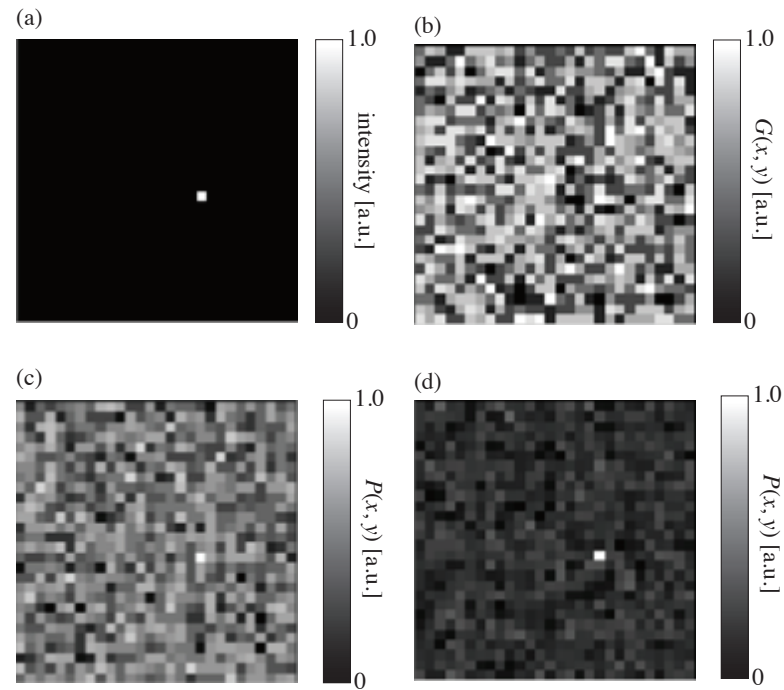

Fig. 3 Simulation results of ghost imaging with deep learning using 5 measurements. (a) sample image, (b) ghost image, (c) ghost image with deep learning $(\mathrm{N}=100)$ and (d) ghost image with deep learning $(\mathrm{N}=200)$. (a)

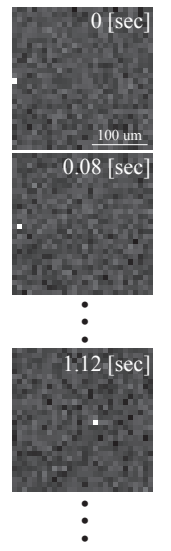

(b)

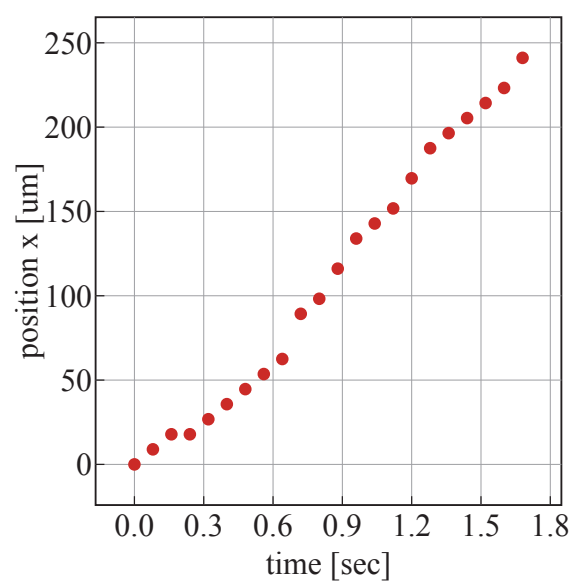

Fig. 4 Experimental results of moving micro-particle detected by ghost imaging with deep learning $(n=5)$. (a) compensated image and (b) time dependent behaviors of moving micro-particle.

[1] T. B. Pittman, Y. H. Shih, D. V. Strekalov, A. V. Sergienko, Phys. Rev. A 52 (1995) R3429.

[2] D. V. Strekalov, A. V. Sergienko, D. N. Klyshko, Y. H. Shih, PRL 74 (1995) 3600.

[3] 14) H. Taguchi, Y. Mizutani, and Y. Takaya, Comp. Opt. Sens. Imag. 2017 (2017) CTh2B.3.

\section{References}




\title{
Automatic rice seed imaging system for varieties classification
}

\author{
Panintorn Prempree*a, Kosom Chaitavon ${ }^{\mathrm{a}}$, Supanit Porntheeraphat ${ }^{\mathrm{a}}$, Kantip Kiratiratanapruk ${ }^{\mathrm{a}}$, \\ Pitchayagan Temniranrat ${ }^{\mathrm{a}}$, Wasin Sinthupinyo ${ }^{\mathrm{a}}$, Anchalee Prasertsak ${ }^{\mathrm{b}}$ \\ ${ }^{a}$ National Electronics and Computer Technology Center (NECTEC), National Science and \\ Technology Development Agency (NSTDA), Pathum thani, 12120, Thailand \\ ${ }^{b}$ Bureau of Rice Research and Development, Rice Department, Bangkok, 10900, Thailand \\ *Email: panintorn.prempree@nectec.or.th
}

\begin{abstract}
Rice seed classification is one of important process for rice breeding since mixing of foreign rice seeds can affect good traits of cultivated rice. A usual method for foreign rice seed identification is visual testing by experts, which is laborious and time consuming. In order to overcome this difficulty, there are many researches related on automatic rice seed classification using image processing and machine learning technique. However, the performance of system based on those techniques depends on number of dominant features that can be extracted from image of rice seeds and number of samples. In this paper, automatic rice seed imaging system is presented. This system can guarantee more than $90 \%$ of proper images suitable for varieties classification. Furthermore, to validate classification performance, the images of three Thai rice varieties (CNT1, PTT1 and RD51) have been captured and tested. The classification accuracy for CNT1, PTT1 and $\mathrm{RD} 51$ are $98 \%, 99 \%$ and $97 \%$ respectively.
\end{abstract}

Keywords: Agriculture, Seed classification, Autonomous system, Image processing, Optical measurement, Machine 1maning

\section{Introduction}

Purity of rice seed is one of important factor for breeding and cultivation. The presence of foreign seed, especially weed rice, can affect productivity yields [1] and good traits of cultivating rice seed. Thailand is one of major agricultural countries which exports huge numbers of agriculture products, especially rice, every year. Currently, there are more than 114 well-known Thai rice varieties [2] that have been cultivated throughout the country. Hence, risk of mixing various species in each cultivation is high. Therefore, rice seed production company or other related organizations (eg. Rice Department) has to ensure rice seeds purity before selling them to farmer. According to Rice Department of Thailand, the foreign rice seeds are identified and removed manually by naked eye of experts based on dominant features of interested rice seeds. This process is laborious and time consuming since the experts need to examine at least 100 grams of rice (approx. 4000 seeds) per production lot. Furthermore, it is difficult to train their skills to next generation due to variation of rice seeds and degradation of rice seeds over time.

With help of current computer vision and machine learning technology, it is possible to capture high resolution rice seed images and then identify their varieties [3]. At presence, there are many researchers working on the topic of rice seed classification and rice seed recognition system based on seed appearance [2]. However, the characteristic of rice seeds is varied with amount of water, climate and other uncontrollable factors. Thus, the developed system and its algorithm need to be updated from time-to-time and require large amount of good quality rice seed image to improve or maintain its accuracy.

In this paper, we propose simple, yet effective, automatic imaging system based on optical set-up and digital camera. This system can capture high resolution rice seed images, which are suitable for further classification.

\section{System Design}

There are various designs of automatic rice seed imaging system [4-5]. However, it is hard to capture dominant features of rice seed unless the rice seeds do not place properly in imaging area as shown in Figure 1. Furthermore, the lighting condition is also important since color is also one of significant and necessary parameter for classification. Therefore, the proposed system has to 
be designed to satisfy given requirements.
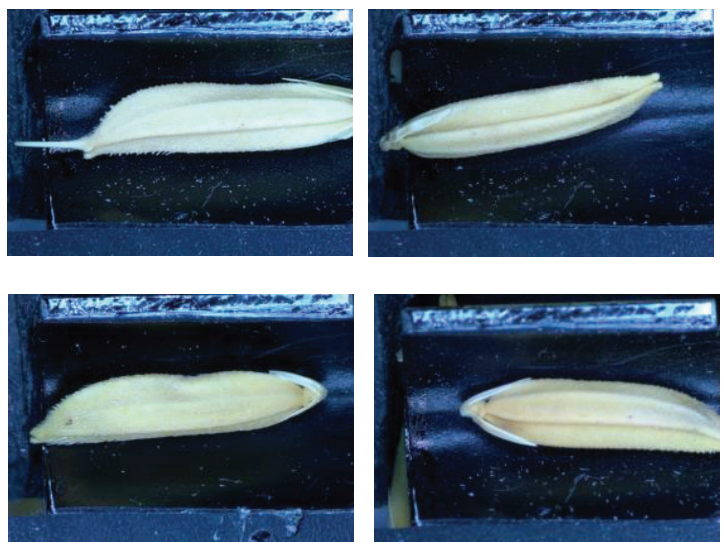

Figure 1. Usuable image due to improper rice seed placing

Our designed system consists of three main parts: 1) Imaging System 2) Rice Feeder 3) Rice seed detection algorithm,

\section{Imaging system}

For lighting condition, ring white rice source is chosen for uniform light distribution in small area since size of rice seed is approximately $2 \times 11 \times 2 \mathrm{~mm}^{3}$. However, reflected light from background can affect color of rice seed and make it difficult for segmentation and classification. Hence, circular polarizer and linear polarizer are added into system to reduce the effect of reflected light. Furthermore, high resolution image with 6000 dpi in $3840 \times 2748$ pixel $^{2}$ can be obtained by combing digital camera with tele-centric lens. Thus, this resolution and lighting condition are sufficient for analyze size, shape, color or even roughness of rice seed, which are key features for rice seed classification.

\section{Rice feeder}

Rice feeder is necessary to convey rice seeds to imaging area. There are many methods such as conveyor [5] or bowl feeder (helical plate with vibrating motor) [4]. In our system, helical plate with vibrating motor is used since it can mostly guarantee that the rice seed will move into imaging area one at a time. However, disadvantage of this approach is the timing and the distance between each entered rice seed is random even if speed of vibration is nearly constant. Thus, optional sensor or image processing for detecting rice seed is needed.

\section{Seed detection algorithm}

Our system is able to detect incoming rice seed automatically based on image processing algorithm. Benefits of using image processing over adding detection sensor are less complexity in both system design and synchronization. To detect rice seed, certain areas of images is marked as region of interest (ROI) as shown in Figure 2. RGB value of pixels inside green ROI and red ROI are averaged (ie. $(\mathrm{R}+\mathrm{G}+\mathrm{B}) / 3$ ) and if the averaged value of green ROI is above setting threshold, then there is a rice seed ready for imaging. Then, system will stop the rice feeder and capture rice seed image. In the other hand, average value in red ROI is for controlling intensity of light.

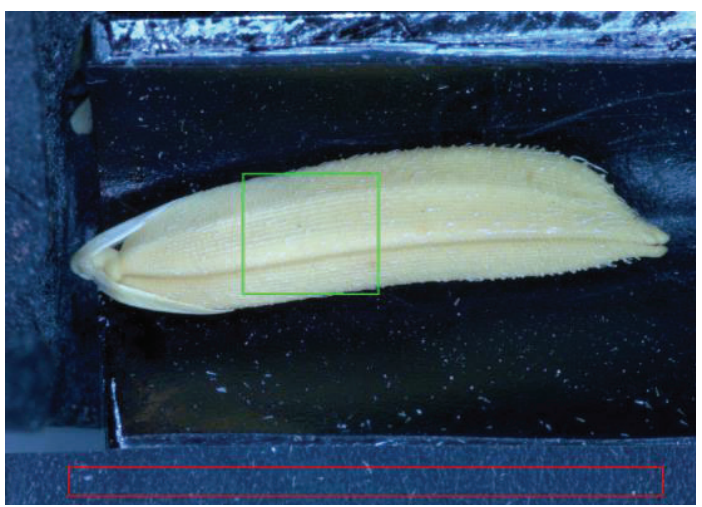

Figure 2. ROI for rice seed detection

By integrating all following parts as shown in Figure 3, our developed system is shown in Fig. 3. The current performance of our system is fully automatic rice seed capture with speed of 10 seeds per minutes. In addition, manual operated system is possible for examining rice seed individually.

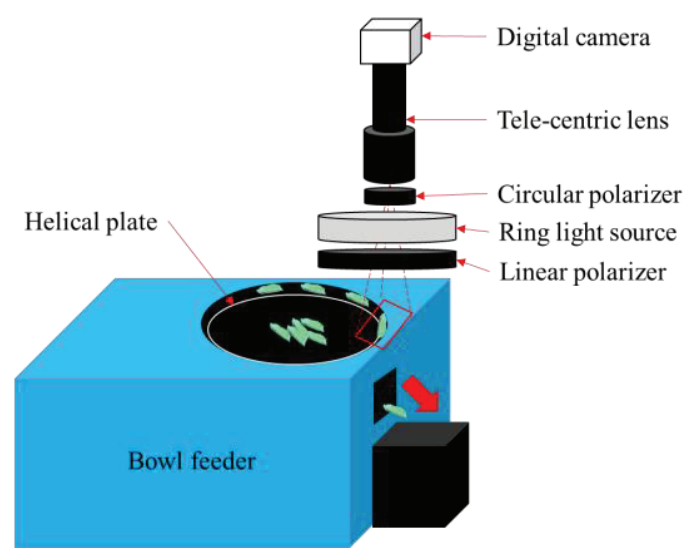

Figure 3. System overview

\section{Result and Discussion}


To validate performance of our system, 6,000 images of three Thai rice varieties (CNT1, PTT1, RD51) have been captured.

\section{Quality of captured rice seed image}

Example of usable images of each rice varieties is shown in Figure 4, which is 5,421 out of 6,000 captured images (approximately 90.35\%).
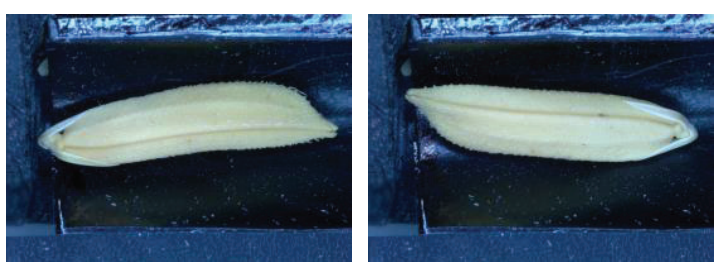

(a)
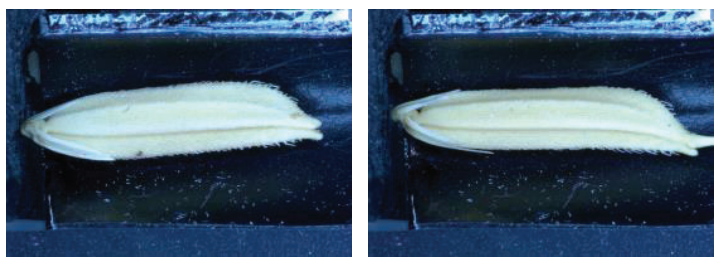

(b)
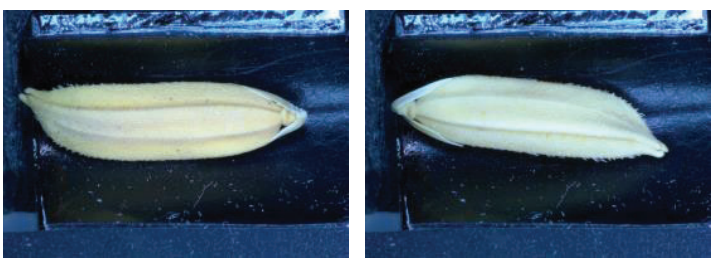

(c)

Figure 4. Example of captured images (a) CNT1 (b) PTT1 (c) RD51

\section{Seed classification}

Rice seed images in obtained in previous section have been pre-processed and trained using Support Vector Machine (SVM) algorithm. Then, 200 rice seed untrained images of CNT1, PTT1 and RD51 each are randomly sampled for classification. The classification accuracy for CNT1, PTT1 and RD51 are 98\%, 99\% and 97\% respectively.

Table 1. Accuracy of CNT1 Model

\begin{tabular}{|l|l|l|}
\hline & CNT1 & Other \\
\hline CNT1 & 198 & 2 \\
\hline PTT1 & 4 & 196 \\
\hline RD51 & 6 & 194 \\
\hline
\end{tabular}

Table 2 Accuracy of PTT1 Model

\begin{tabular}{|l|l|l|}
\hline & PTT1 & Other \\
\hline CNT1 & 0 & 200 \\
\hline PTT1 & 196 & 4 \\
\hline RD51 & 1 & 199 \\
\hline
\end{tabular}

Table 3 Accuracy of RD51 Model

\begin{tabular}{|l|l|l|}
\hline & RD51 & Other \\
\hline CNT1 & 0 & 200 \\
\hline PTT1 & 0 & 200 \\
\hline RD51 & 184 & 16 \\
\hline
\end{tabular}

Performance of our designed automatic rice seed imaging system is good in term of quality of captured images and classification. In addition, this system can be applied to measure rice grain embryo's size, size measurement and glutinous rice separation, which are our previous research [6]. However, for achieving more than $90 \%$ of usable images, the capturing speed is limited to only 10 samples per, which is too slow for industrial production line but it is still acceptable for rice breeding research.

\section{Acknowledgement}

The author would like to the Rice Department of Thailand for providing funding of this project and supplying rice seed samples, and National Electronics and Computer Technology Center (NECTEC) for supplying equipment and facilities.

\section{References}

[1] A.A. Aznan, I.H. Rukunudin, A.Y.M. Shakaff, R. Ruslan, A. Zakaria and F.S.A. Saad. "The use of machine vision technique to classify cultivated rice seed variety and weedy rice seed variants for the seed industry". Int. Food Research Journal, vol 23(suppl), pp. S31-S35, 2016.

[2] B, Lurstwut. C. Pornpanomchai. "Application of Image Processing and Computer Vision on Rice Seed Germination Analysis.” (2016).

[3] Phan, Hong \& Vo, Ta-Hoang \& Tran, Thanh-Hai \& Le, Thi \& Nguyen, Thuy \& Hai, Vu. (2015). Comparative 
Study on Vision Based Rice Seed Varieties Identification.

10.1109/KSE.2015.46.

[4] Marschalek, Rubens \& Cesar Silva, Mauricio \&

Batista dos Santos, Samuel \& Ricardo Manke, Johnny \&

Bieging, Carlos \& Porto, Geovani \& Wickert, Ester \& De

Andrade, Alexander. (2017). Image - Rice Grain Scanner:

A three-dimensional fully automated assessment of grain size and quality traits. Crop Breeding and Applied

Biotechnology. $\quad 17 . \quad 89-97 . \quad 10.1590 / 1984-$ 70332017v17n1s15.

[5] I.Chatnuntawech et al, "Rice Classification Using Spatio-Spectral Deep Convolutional Neural Network", arXiv:1805.11491v2

[6] K. Chaitavon et al, "Development if photonics-based physical quality assessment systems for rice seeds and grains", IRC14-0592, 4th International Rice Congress, 27 October - 1 November 2014. 


\title{
Light-source color correlation of wide-field spectroscopic imaging for the adaption to spatial and temporal variations when using an unmanned aerial vehicle
}

\author{
Kotone YOKOYAMA*, Natsumi KAWASHIMA, Tomoya KITAZAKI, Sora MIZUTANI, \\ Hanyue KANG and Ichiro ISHIMARU
}

Faculty of Engineering, Kagawa University, 2217-20 Hayashi, Takamatsu, Kagawa, 761-0396, Japan e-mail: ishimaru@eng.kagawa-u.ac.jp HP: http://www.eng.kagawa-u.ac.jp/ ishimaru/index.html

\begin{abstract}
Wide-area spectroscopic imaging by an unmanned aerial vehicle has been proposed for the early detection of red tides. To ensure the accurate determination of the chlorophyll concentrations of plankton, the spectral reflectance of internally diffused light was calculated separately from changes in the light source (sunlight), due to the time and location. The proposed one-shot spectroscopic line imager was set at Brewster's angle and p- and s-analyzers were set alternately in each pixel. In a p-polarized pixel, the internally reflected light and surface-reflected light are detected. Surface-reflected light has the color of the light source. In an s-polarized pixel, only internally diffracted light is measured. The color of the light source can thus be estimated from the difference of the two spectral intensities. Additionally, we proposed near cross-Nicole analysis to compensate for the lack of the dynamic range of two-dimensional array devices. This study demonstrated the feasibility of the proposed method for measurements taken at a lake. We were able to identify the specific absorbance peak (at a wavelength of $680 \mathrm{~nm}$ ) from corrected spectral relative intensities.
\end{abstract}

\section{Introduction}

We aim to realize the early detection of red tides, which result from plankton plagues, using our ultracompact spectroscopic apparatus mounted on an unmanned aerial vehicle; i.e., a drone. The number of outbreaks of red tide in the Seto Inland Sea has gradually decreased year by year since the 1970s. However, losses of fishery dams relating to red tides amount to hundreds of millions of yen annually. The early detection of red tides would allow forestry and fishery workers to be alarmed and floating fishery cages to be removed in time. Several tens of species of plankton include chlorophyll in photosynthesis. Chlorophyll absorbs visible light and generates nutritive components, and the spectral absorbance of chlorophyll at wavelengths of visible light is thus well known. Therefore, for the monitoring of red tides, chlorophyll concentrations in oceans are evaluated from spectral absorbance data acquired by artificial satellites. However, the temporal and spatial resolutions of such data are not sufficient for rapid onsets. It is thus expected that multicopter and fixed-wing drones will be used to measure chlorophyll concentrations with high frequency. In this case, however, light-source colors that vary from hour to hour and place to place need to be corrected temporally and spatially. We thus propose a light-source color correction method using Fresnel's equations for reflection. In this proposed method, specular light reflected from the sea surface, which has the same color as the light source, and light diffracted from within the ocean are physically separated and measured at the same moment. Employing this method, a developed one-shot spectroscopic imager [1]-[5], which is set at Brewster's angle, detects p-polarized beams (internally reflected light) and s-polarized beams (surface and internally reflected light) separately. We obtain lightsource colors as surface-reflected light analytically. Additionally, the spectral reflectance of internally diffracted light can be calculated to estimate the chlorophyll concentration.

This paper demonstrates the feasibility of correcting light-source colors and overcoming a wide range of sunlight illuminance employing near open-Nicole analysis. 


\section{Background correction with simultaneous detection of surface-reflected light for the light- source color and internally diffracted light using two polarized beams observed from Brewster's angle}

\section{Principle of light-source color correction [6][7]}

Figure 1 shows that we set two types of analyzer in the apertural areas of multiple slits. Ocean surfaces are illuminated by diffused illumination because natural sunlight is diffracted and scattered in air. Furthermore, light is depolarized through the reflection of internally diffracted light from internal components.

To improve the clarity of an interferogram, we set the multiple slits on the conjugate optical objective plane [3][4]. The inclination angle of the hyperspectral camera is set as Brewster's angle and the surface reflectance of $p$ polarized beams is thus zero. Beams passing through ppolarization filters are internally diffracted reflections while beams passing through s-polarization filters are the sum of light having the color of the light source, such as the color of natural sunlight, and internally diffracted reflections. We can easily separate the surface reflection and internal diffraction from these two detected beams.

However, the intensity ratio between the surface reflection and internal diffraction is around 1000 . Therefore, these two types of polarized beams cannot be detected using one two-dimensional light-receiving array device because of the poor dynamic range. To solve this issue of the dynamic range, the polarization angle as an spolarized filter is set as a small inclination angle $\varphi$ according to Malus' law. In this case, the detected light ratio of surface-reflected light reduces in accordance with cosine $\varphi$. Considering the reduced ratio, we can separate surface-reflected light as light having the color of the light source and internally diffracted light.

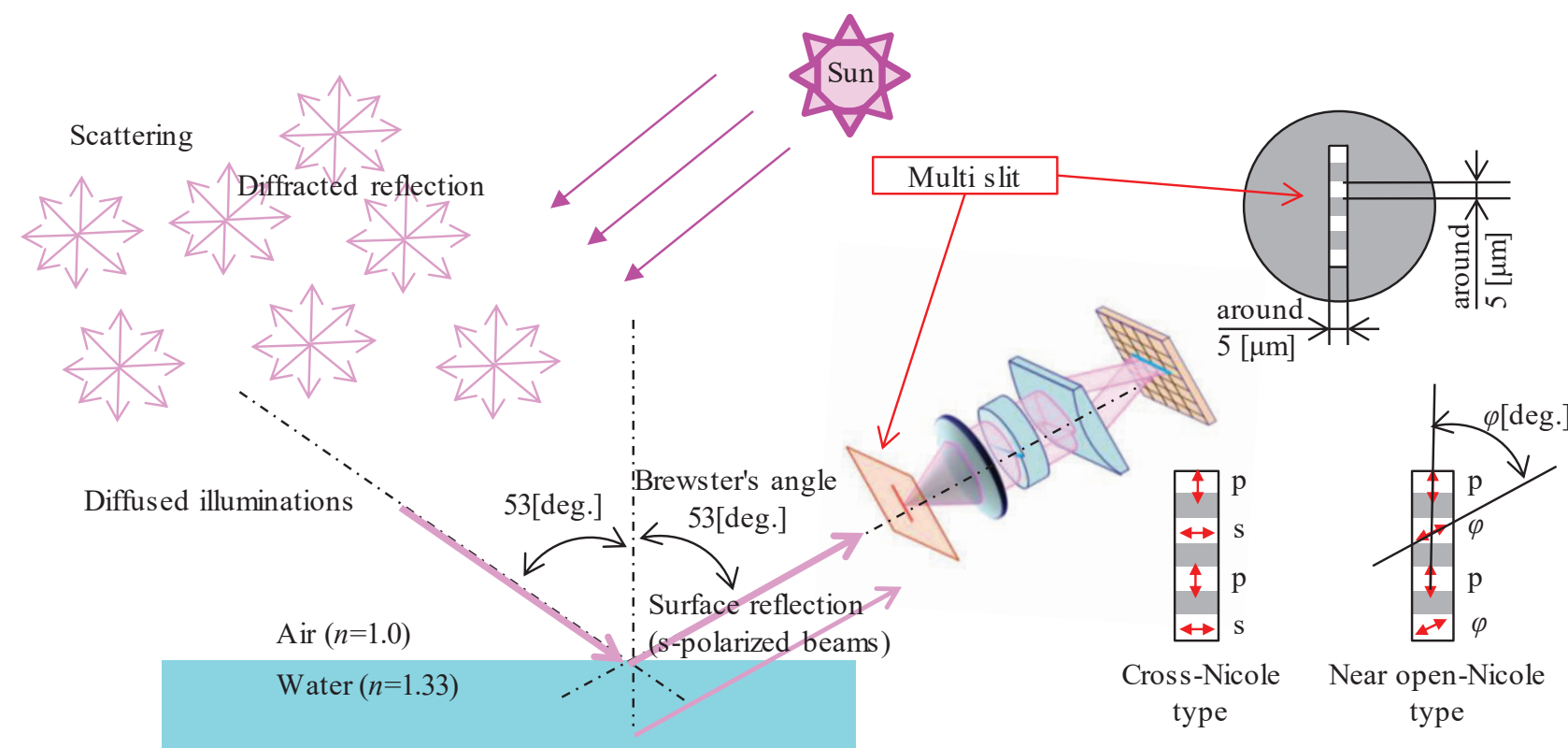

Internal diffracted lights

(depolarized lights: $s+p-p o l a r i z e d ~ b e a m s)$

Analyzer in each apertural area of multi slit

Figure 1 Schematic diagram of the background correction method with the simultaneous detection of surface-reflected light having the light-source color and internally diffracted light through the observation of two polarized beams at Brewster's angle [6][7].

2. Experimental results for identifying chlorophyll spectral absorbance $(680 \mathrm{~nm})$ at a lake

In this feasibility experiment, we observed the lake Saburo-ike, located near our university. A one-shot Fourier spectroscopic imager was set at Brewster's angle
(53 degrees). The red solid line in Figure 2(a) shows the recording of internally diffracted light as a p-polarized beam. Internally reflected light and surface-reflected light were detected by an s-polarization analyzer as shown by the blue solid line. In Fig. 2(b), we discriminate the 
specific spectral absorbance of chlorophyll (at a wavelength of $680 \mathrm{~nm}$ ). Additionally, we recognize the color of the lake water, which was green, as having high relative intensity around 500-600 $\mathrm{nm}$.

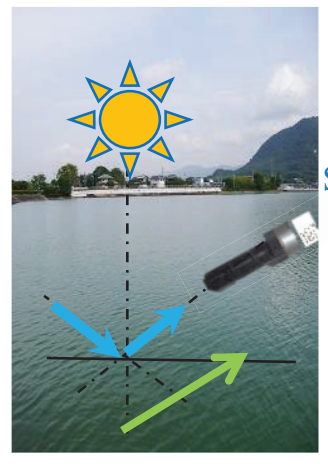

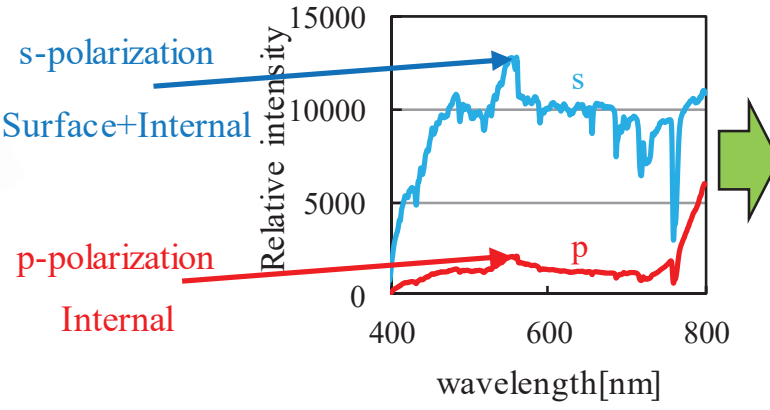

(a)Spectral intensity

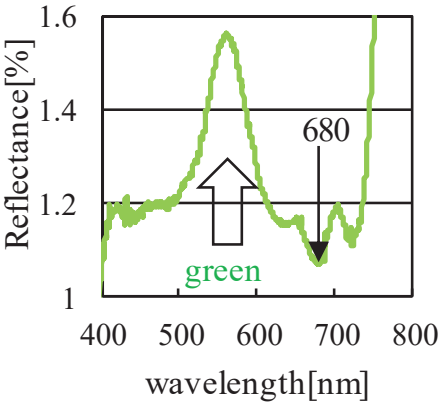

(b)Internal spectral reflectance

Figure 2 Results of an experimental demonstration of the light-source correction method at a lake.

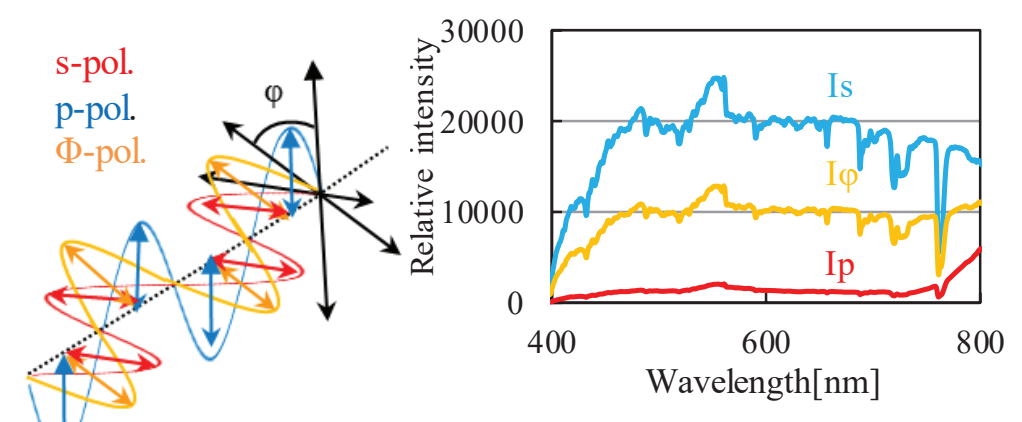

(a) Spectral intensity (Ip, Is, I $\varphi$ )

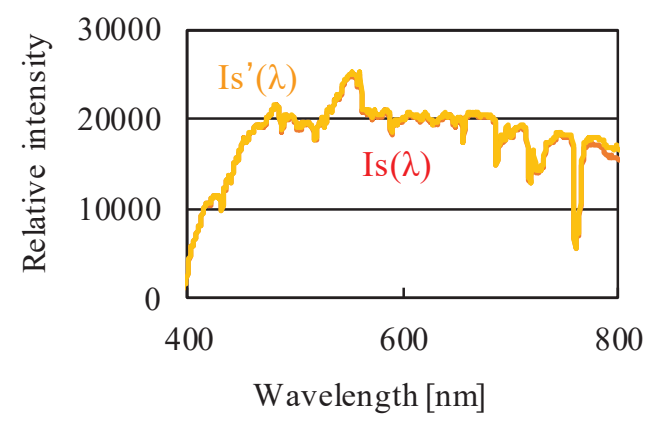

(b) Corrected spectrum

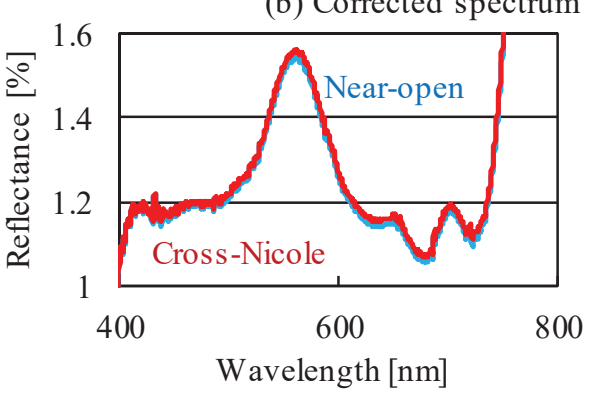

(c) Spectral internal reflectance

Figure 3 Experimental results of the near open-Nicole method for expanding the dynamic range of a two-dimensional array device to adapt for the wide range of sunlight intensity.

As shown in Figure 3, we verified near open-Nicole analysis for expanding the dynamic range of a twodimensional array device to a wide range of natural sunlight intensity. We set a p-analyzer at zero degrees and a $\varphi$ analyzer at 43 degrees for the near open-Nicole condition. In Fig. 3(a), the spectroscopic relative intensities of the p-polarized beam $I_{P}(\lambda)$, s-polarized beam $\mathrm{I}_{\mathrm{S}}(\lambda)$, and $\varphi$-analyzer $\mathrm{I}_{\varphi}(\lambda)$ are presented as solid lines.
Figure 3(b) shows the spectral relative intensities $\mathrm{I}_{\mathrm{S}}(\lambda)$ and $\mathrm{IS}_{\mathrm{S}}^{\prime}(\lambda)$ calculated using the near open-Nicole correction algorithm based on Malus' law. The calculated internally diffracted spectral reflectances of cross-Nicolle $I_{S}(\lambda)$ and near open-Nicole $I_{P}(\lambda)$ are shown in Fig. 3(c). As shown in Fig. 3(b), the calculated $\mathrm{I}_{\mathrm{s}}{ }^{\prime}(\lambda)$ agrees with the measured value $I_{S}(\lambda)$. These two types of internally 
diffracted reflectance are equivalent as shown in Fig. 2(c), and we thus verify the near open-Nicole method.

\section{Conclusions}

We proposed and demonstrated a light-source color correlation method for the wide-area visualization of plankton distributions, using an ultra-compact spectroscopic imager mounted on a drone. We were able to distinguish the specific spectral absorbance of chlorophyll (at a wavelength of $680 \mathrm{~nm}$ ) from spectra recorded at a lake.

In future work, we will equip drones with our proposed ultra-compact one-shot spectroscopic line imager and verify the effectiveness of the imager in the highfrequency monitoring of red tides.

\section{References}

[1]T.Uraki,, I.Ishimaru, "Feasibility Demonstration of Real-Time Measurement by One-shot Compact Fourier Spectroscopic Imaging”, Proc. of 8th JapanFrance Congress on Mecatronics, pp.549-553 (2010)

[2] S. Sato, M. Fujiwara, S. Suzuki, A. Nishiyama, I. Ishimaru, "Proposal of one-shot-type spectroscopictomography for non-invasive medical-measurement", Proc. of OSA-SPIE European Conferences on Biomedical Optics 2013, Vol. 8798, pp.87980I-1$8(2013)$

[3] Shun Sato, Wei Qi, Natsumi Kawashima, Kosuke Nogo, Satsuki Hosono, Akira Nishiyama, Kenji Wada, and Ichiro Ishimaru, "Ultra-miniature one-shot Fourier-spectroscopic tomography," Optical Engineering, Vol.55, pp. 025106-1 - 025106-8 (2016)

[4] Natsumi Kawashima; Kosuke Nogo; Satsuki Hosono; Akira Nishiyama; Kenji Wada; Ichiro Ishimaru, "Sensitivity improvement of one-shot Fourier spectroscopic imager for realization of noninvasive blood glucose sensors in smartphones", Opt. Eng. 55(11) 110506 doi: 10.1117/1.OE.55.11.110506 (2016)

[5] Ichiro ISHIMARU, Natsumi KAWASHIMA, Satsuki HOSONO, "Built-in hyperspectral camera for smartphone in visible, near-infrared and middleinfrared lights region (1st. report) - Trial products of beans-size Fourier-spectroscopic line-imager and feasibility experimental results of middle infrared spectroscopic imaging - “, Proc. SPIE 9855, NextGeneration Spectroscopic Technologies IX, 985504 (2016)

[6] Satsuki HOSONO, Natsumi KAWASHIMA, Dirk Wollherr and Ichiro ISHIMARU, Built-in hyperspectral camera for smartphone in visible, nearinfrared and middle-infrared lights region (3rd. report) - Spectroscopic imaging for broad-area and real-time componential analysis system against local unexpected terrorism and disasters -, Proceedings Volume 9855, Next-Generation Spectroscopic Technologies IX; 985506 (2016)

[7] Satsuki Hosono, Kengo Aizawa, Tsubasa Saito, Mizuho Okada, Kosuke Nogo, Natsumi Kawashima, Kuninao Tada, and Ichiro Ishimaru、 "Quantitative Evaluation of the Object Color without Influence from the Light Source Color under Unstructured Environment -A Background Correction Method Using Polarization Properties-", Light, Energy and the Environment OSA Technical Digest (online) (Optical Society of America, 2016), paper JW4A.22 ISBN: 978-0-9600380-4-6 (2016) 


\title{
Systematic error correction for phase detection in sinusoidal frequency modulation displacement measuring interferometer
}

\author{
Masato Higuchi*a, Dong Wei ${ }^{\mathrm{a}}$, Masato Aketagawa ${ }^{\mathrm{a}}$ \\ a'Department of Mechanical Engineering, Nagaoka University of Technology, 1603-1, \\ Kamitomioka Nagaoka, Niigata 940-2188, Japan
}

\begin{abstract}
A sinusoidal frequency modulation (SFM) interferometer is a good candidate for high accuracy displacement measurement. It can directly adopt a frequency stabilized light source based on frequency modulation spectroscopy, which light source frequency is also modulated sinusoidal way. To achieve a high resolution, a method detecting displacement from the interference signal is an important. A SPPE using a conventional phase-locked loop (PLL) is expected to have a capability of high resolution, while it have a problem that displacement results have systematic errors with a period of quarter wavelength depending on modulation indexes on interferometers. We propose a SFM interferometer with PLL with successive error correction to achieve high accuracy and high resolution. In this paper, we discuss about displacement measurement using the proposed PLL.

Keywords: displacement measurement, sinusoidal frequency modulation, sinusoidal phase modulation, phase-locked loop, modulation index

\section{Introduction}

In the nanotechnology field such as ultra-precise processing and semiconductor manufacturing, a positioning is conducted precisely in an order of sub nano meter. The positioning accuracy must be based on a definition of meter and an optical interferometer with corrected light source such as iodine frequency stabilized He-Ne laser, is widely used.

A sinusoidal frequency modulation (SFM) interferometer is a good candidate for high accuracy displacement measurement. It can directly adopt a frequency stabilized light source based on frequency modulation spectroscopy, which light source frequency is also modulated sinusoidal way. A SPPE achieves a high resolution displacement measurement. The SPPE demodulates a displacement with PLL and is introduced in grating interferometer measuring displacement based on grating pitch. For high accuracy and high resolution displacement measurement, combination of SFM interferometer and PLL for displacement demodulation is important. In these technique, a modulation index is an important factor. In SFM interferometer, it is a function of an initial optical path difference between a measurement and a reference arm, and a frequency excursion, and often varies in any practical interferometer. In SPPE, the

modulation index causes a systematic error in demodulation and it must be a specific value and fixed.

In this study, we develop a PLL demodulation method for SFM interferometer with arbitral modulation index, to achieve both high accuracy and high resolution displacement measurement. In this report, we demonstrate the feasibility of the proposed PLL on sinusoidal phase modulation (SPM) interferometer and discuss about a source of residual systematic errors. The SPM interferometer has similar characteristics compared to the SFM interferometer, and can be set its modulation index by electro-optic modulator (EOM). For our experiment, the SPM interferometer is more suitable than SFM interferometer, designing arbitral modulation index conditions.

\section{Measuring principle}

\section{Experiment}

Fig.1 shows an experimental set up. The interferometer is an unbalanced Mach-Zehnder type. A beam from the He-Ne laser is split into two beams by a first BS; these are a reference and a measurement beam. The phase of the measurement beam is modulated sinusoidal way through the EOM. These beams are combined by the second BS and then interfere. An interference signal from the PD and modulation indexes are represented as Eq. (1), (2),
\end{abstract}




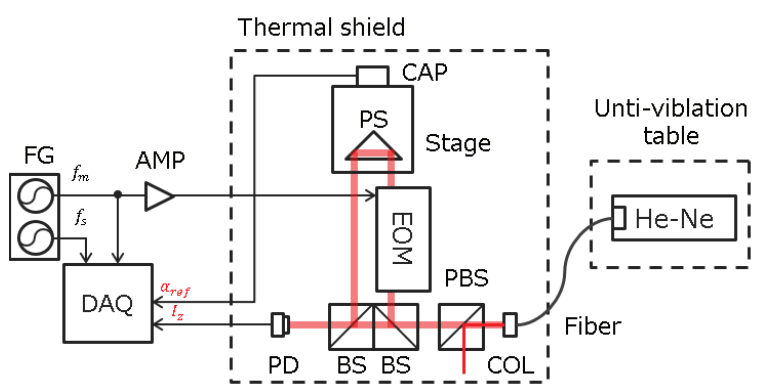

Figure 1. Experimental setup, COL: Collimator, PBS: Polarized beam splitter, BS: Beam splitter, EOM: Electro optic modulator, PS: Prism, CAP: Capacitive displacement sensor, PD: Photo detector, AMP: High voltage amplifier, DAQ: Data acquisition devices, FG: Function generator

$I_{z} \propto S_{a}+S_{b} \cos \left(\alpha-m_{S P M} \sin \omega_{m} t\right)$,

$m_{S P M}=\frac{\pi}{V_{\pi}} V_{E O M}$

$\alpha=\frac{2 \pi}{\lambda / 2} \Delta z$

where, $S_{a, b}, \alpha, \omega_{m}, \lambda$ and $\Delta z$ are amplitudes of dc and ac component, a phase angle from displacement, angular modulation frequency, wavelength and displacement, respectively. The displacement of the PS is demodulated using the PLL by the interference signal.

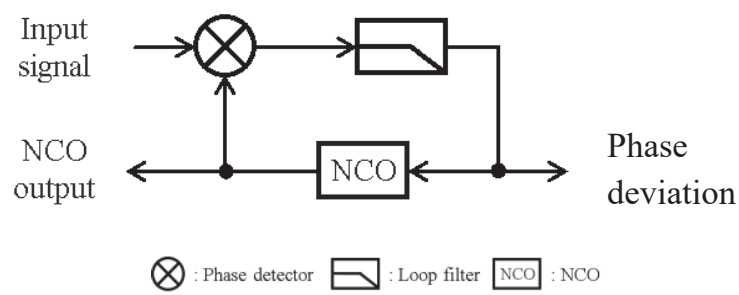

Figure 2. Conventional PLL

Fig. 2 shows the conventional PLL. The PLL is composed with phase detector, loop filter and numerical controlled oscillator (NCO). The phase detector and loop filter detect the phase deviation between an input signal and an output of the NCO. The error signal is then fed back to the NCO. The NCO leads or lags its phase angle to lock the NCO output into input signal. In the displacement measurement, the input signal is rewritten in following Eq. (3) and (4),

$$
\begin{aligned}
& I_{z} \times \cos \left(0.5 \omega_{m} t\right)=S_{a} \cos 0.5 \omega_{m} t+ \\
& \quad \cdots+S_{b} S_{c} \sin \left(2.5 \omega_{m} t+\beta\right)+\cdots, \\
& \quad \beta=\operatorname{atan}\left\{\frac{J_{3}}{J_{2}} \tan \alpha\right\},
\end{aligned}
$$

where, $S_{b, c}, \beta$ and $J_{n}$ are amplitudes of harmonics, the phase angle of $2.5^{\text {th }}$ harmonic term and $n^{\text {th }}$ order Bessel function. In this paper, the $2.5^{\text {th }}$ harmonic is used for the demodulation and $\mathrm{NCO}$ frequency is $2.5 \omega_{m} t$ to phase lock to the $2.5^{\text {th }}$ harmonic term.

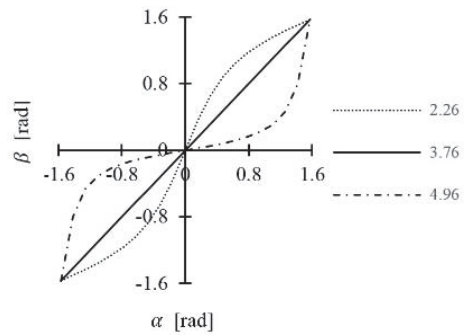

Figure 3. Systematic error, $\alpha$ is the phase angle from displacement, $\beta$ is the phase angle demodulated by the PLL

The phase angle $\beta$ is demodulated by integrating the phase deviations in the PLL and is not directly represent the phase angle from the displacement. Figure 3 show relationship between phase angles from the displacement and demodulated by the PLL. In the arbitral modulation index conditions 2.26 and $4.96 \mathrm{rad}$, for example, the relationship is as an arc tangent causing systematic error in the measurement. The proposed PLL successively calculate the phase angle $\alpha$ from the phase angle $\beta$ by Eq. (4).

\section{Displacement measurement}

We demonstrate displacement measurements using the proposed method.

Table 1. Experimental conditions

\begin{tabular}{ll}
\hline Modulation frequency & $50 \mathrm{kHz}$ \\
Modulation index & $1.0,2.0,3.0,3.8,4.0 \mathrm{rad}$ \\
Wavelength & $632.8 \mathrm{~nm}$ \\
\hline Sampling frequency & $600 \mathrm{kHz}$ \\
Sample number & 650000 points $(\approx 1 \mathrm{~s})$ \\
\hline PS displacement & Sinusoidal, 1.3 $\mu \mathrm{m}, 2$ preiods \\
\hline In measurements, interference signals for each \\
modulation indexes are obtained separately and then are
\end{tabular}


demodulated on computer-based calculation. Concurrently, the capacitive sensor measures the displacement as a reference. Table 1 show the experiential conditions.

\section{Results and Discussion}

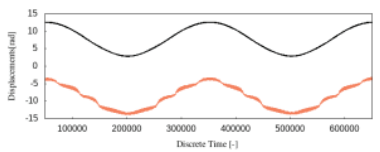

(a) $1.0 \mathrm{rad}$

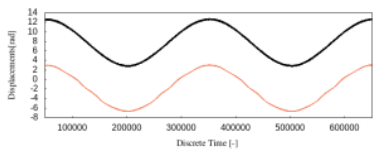

(b) $2.0 \mathrm{rad}$

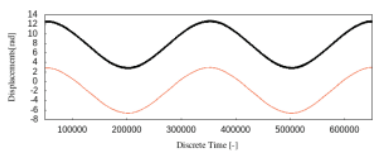

(c) $3.0 \mathrm{rad}$

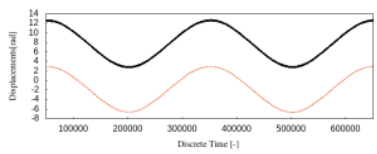

(d) $3.8 \mathrm{rad}$

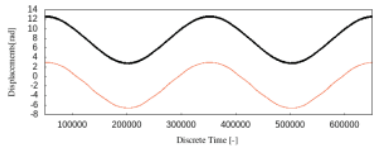

(e) $4.0 \mathrm{rad}$

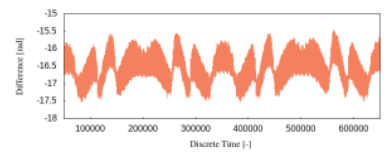

(f) $1.0 \mathrm{rad}$

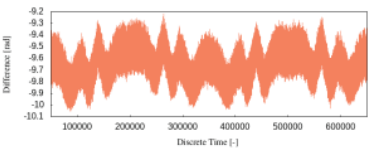

(g) $2.0 \mathrm{rad}$

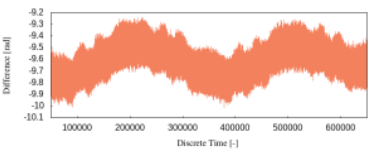

(h) $3.0 \mathrm{rad}$

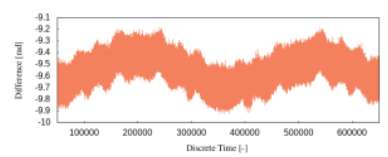

(i) $3.8 \mathrm{rad}$

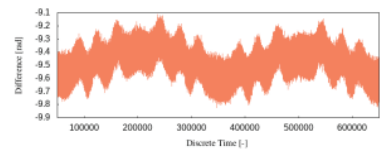

(j) $4.0 \mathrm{rad}$
Figure 4. Measurement results, phase angle displacements and phase differences

Figure 4 show measurement results. Figure 4 (a) (b) are displacements from demodulated by the proposed PLL (red line) and obtained from reference sensor (black line). In each modulation index, the displacement has a same waveform compared to reference. This reveals the systematic error correction in the proposed method works. Figure 4 (f) $\sim(\mathrm{j})$ show phase displacement differences between the proposed PLL and the reference. Almost two parts of waveform can be observed; one is long term with two periods and another is short term especially seen in Figure 4 (f). The long term waveform is caused by frequency difference between actual and calculated, Abbe offset from measurement axes of the interferometer and reference sensor. The short waveform is the residuals of the systematic error.

\section{Conclusion}

We demonstrate the feasibility of the proposed PLL on sinusoidal phase modulation (SPM) interferometer. The displacement is demodulated in arbitral modulation indexes.

\section{References}

A. Dandridge, L. Goldberg, "Current-induced frequency modulation in diode lasers", ELECTRONICS LETTERS, pp. 302-304, No. 7, Vol. 18, (1982).

W. G. Schweitzer, Jr., E. G. Kessler, Jr., R. D. Deslattes, H. P. Layer, and J.R. Whetstone, "Description, Performance, and Wavelength of Iodine Stabilized Lasers," Applied Optics, Vol. 12, No.12, December (1973).

O. Sasaki and H. Okazaki, "Sinusoidal phase modulating interferometry for surface profile measurement," Applied Optics, Vol. 25, No. 18, 15 September 1989.

K. Isleif, O. Gerberding, T. S. Schwarze, M. Mehmet, G. Heinzel and F. G. Cervantes, "Experimental demonstration of deep frequency modulation interferometry," Optics Express 1676, DOI:10.1364/OE.24.001676, Vol. 24, No. 2, 25 January 2016.

Vu, T., Higuchi, M., Aketagawa, M., "Accurate displacement-measuring interferometer with wide range using an $\mathrm{I} 2$ frequency-stabilized laser diode based on sinusoidal frequency modulation," Meas. Sci. Technol. 27105201 (10pp) (2016).

T. Ohara, et al, "Scanning Probe Position Encoder(SPPE)a new approach for a high precision and high speed position measurement system," SPIE 4344, Metrology, Inspection, and Process Control for Microlithography XV, 552 (August 22, 2001); doi:10.1117/12.436778. 


\title{
An optical angle sensor based on second harmonic generation of a mode-
}

\author{
locked laser \\ Hiraku Matsukuma*, Shuhei Madokoro, Masaru Nakao, Yuki Shimizu, Wei Gao \\ Department of Finemechanics, Graduate School of Engineering, Tohoku University, \\ 6-6-01 Aramaki-Aza-Aoba, Aoba-ku, Sendai, 980-8579, Japan
}

\begin{abstract}
This paper presents an optical angle sensor using characteristics of second harmonic generation (SHG) which is a nonlinear process of electromagnetic field. In a SHG process, the fundamental wave is made incidence into a non-linear optical crystal. The second harmonic wave which has double frequency of the fundamental wave is then generated. The light intensity of the generated second harmonic wave changes with the incident angle of the fundamental wave to the non-linear optical crystal due to phase mismatch which is a factor of determining the conversion efficiency of SHG. The dependence of second harmonic generation on angular displacement is calculated theoretically for mode-locked femto second laser light source. Experiment is also carried out in order to demonstrate the theoretical calculation.
\end{abstract}

Keywords: Angle sensor, Second harmonic generation, Non-linear process, Mode-locked laser, Femto-second laser

\section{Introduction}

Ultra-precision products such as optical instruments and semiconductor devices are composed of parts with a variety of geometries from fundamental flat and/or spherical shapes to the complex free-form surfaces. Many of such parts are required to be manufactured in a highprecision up to sub-micrometer or even nanometer. Due to the tight tolerances in nanomanufacturing, nanometrology of the manufactured parts is of highpriority for quality control of the parts as well as for process control of nanomanufacturing [1]. Nanopositioning and the related nanometrology are therefore necessary for this purpose [2].

In this paper, a new method of angular measurement is proposed utilizing a characteristic of second harmonic generation (SHG). Both theoretical calculations and experimental demonstration are presented.

\section{Principle of angle measurement}

SHG is a non-linear optical process which is proportional to square of light intensity. In a SHG process, the incident wave is focused onto a non-linear optical crystal. The second harmonic wave which has double frequency of the incident wave is then generated. The light intensity of the generated second harmonic wave changes with the angle between the incident wave and the non-linear optical crystal. Here, the incident light is called a fundamental wave. Figure 1 shows a schematic diagram of generation of the second harmonic. The light intensity $I_{2}$ of the second harmonic can be written as follows [3].

$I_{2}=\frac{8 \pi^{2} \mu_{0} c d_{\mathrm{eff}}^{2} L^{2}}{n_{1}^{2} n_{2} \lambda^{2}} \operatorname{sinc}^{2}\left(\frac{\Delta k(\theta) L}{2}\right) I_{1}^{2}$

, where $\mu_{0}$ and $c$ are the magnetic permeability and light velocity in vacuum, $d_{\text {eff }}$ is the effective non-linear coefficient, $n_{1}$ and $n_{2}$ are the refractive indices of the fundamental wave and the second harmonic wave, $L$ is the optical path length in the crystal, and $I_{1}$ represents the fundamental wave intensity. $k(\theta)$ is called the phase mismatch and is expressed by the following equation:

$\Delta k(\theta)=\frac{4 \pi}{\lambda}\left(n_{1}-n_{2}(\theta)\right)$

Since the femtosecond laser has a high peak intensity $I_{1}$, it is possible to obtain a second harmonic wave of high

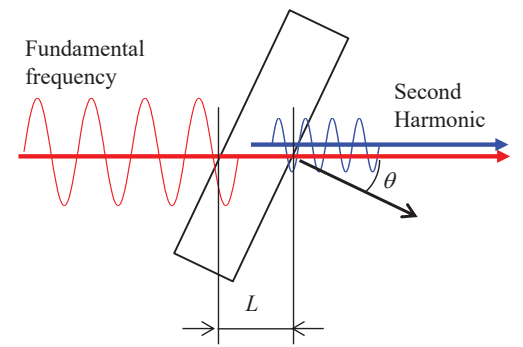

Figure 1 A schematic of second harmonic generation. 
output even with a low average output as compared with a conventional laser light source.

\section{Theoretical calculation}

A light intensity of about $1 \mathrm{MW} / \mathrm{cm}^{2}$ is necessary for SHG. Therefore, when a femto-second laser of about 0.1 $\mathrm{nJ}$ per pulse is used, it is necessary to focus fundamental wave in the crystal by using a lens. The characteristics of SHG power for focusing fundamental wave incidence is expressed by the following equation [4].

$$
P_{2} \propto\left(\frac{b}{L}\right)\left|\int_{-L / b}^{L / b} \frac{\exp (i b \Delta k(\theta) \tau / 2)}{1+i \tau} d \tau\right|^{2}
$$

Here, $b$ is the Rayleigh length of the focused beam, $f$ is the focal length of the focusing lens, $D$ is the incident beam diameter, and $w_{0}$ is the light spot diameter. Because the intensity of second harmonic wave depends on the Rayleigh length, calculation was performed three different focal lengths of lens. Figure 2 shows the calculated results of the angular displacement dependence of the SHG. Here, the calculation was performed assuming that the wavelength of the fundamental wave is $1560 \mathrm{~nm}$ and the crystal thickness is $2 \mathrm{~mm}$.

\section{Experiment}

In order to demonstrate the principle of the angle measurement based on the proposed method, experiments were conducted with the constructed optical system. Figure 3 shows a schematic of the experimental setup. A femtosecond laser pulse (pulse width: $150 \mathrm{fs,} \mathrm{wavelength}$ band: $1560 \mathrm{~nm} \pm 20 \mathrm{~nm}$ ) was collimated to $3 \mathrm{~mm}$ in diameter and then focused onto a non-linear optical (Type I BBO crystal). By a polarizer whose transmission axis was set in $X$-direction, the fundamental wave was cut in

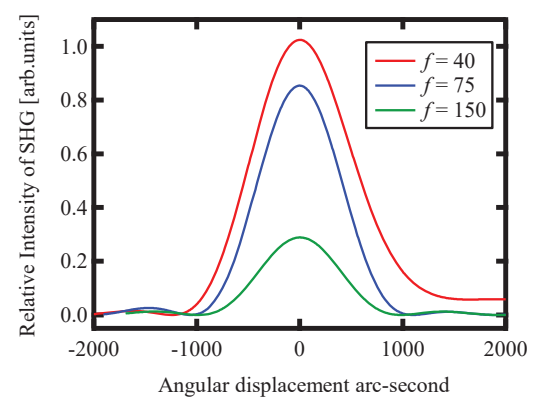

Figure 2 Simulated second harmonic light power

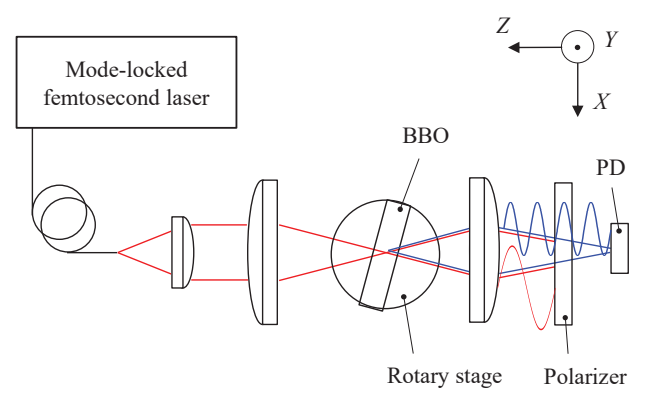

Figure 3 A schematic of the developed experimental setup.

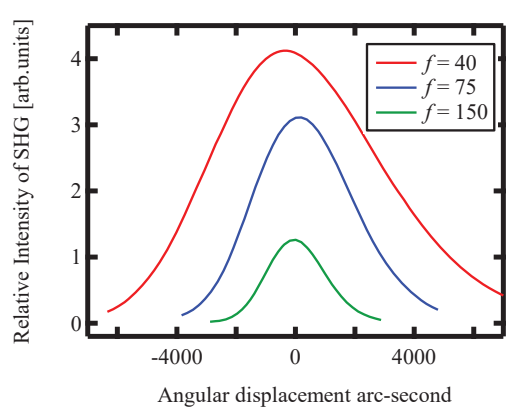

Figure 4 Experimental second harmonic light power dependence on angular displacement

front of the photodiode. The second harmonic wave was made incidence on the photodiode. By measuring the output voltage of the photodiode, the output of the SHG was measured. Figure 4 shows the results of SHG intensity dependence on the angular displacement of the BBO crystal. Here three types of focusing lenses $(f=40$ $\mathrm{mm}, 75 \mathrm{~mm}$ and $150 \mathrm{~mm}$ ) were used. It was confirmed that the tendency of the second harmonic generation output shows similar tendency with the theoretical curve.

This research is supported by Japan Society for the Promotion of Sciences (JSPS) KAKENHI.

\section{References}

[1] Gao, W., et al, "Measurement technologies for precision positioning”, CIRP Ann. Manuf. Techn. 64: 773-796 (2015).

[2] Gao, W., [Precision Nanometrology: Sensors and Measuring Systems for Nanomanufacturing], Springer (2010).

[3] Boyd, R. W., [Nonlinear Optics Third Edition], Academic Press (2008).

[4] Boyd, G. D., Kleinman, D. A., "Parametric Interaction of Focused Gaussian Light Beams”, J. Appl. Phys., 39(8), 3597-3639 (1968). 


\title{
Machine learning for rapid adaptation to individual optical differences for noninvasive blood glucose sensor using mid-infrared
}

\section{spectroscopy*}

\author{
Jyunya Iwaki*, Natsumi Kawashima, Naoyuki Yamamoto, Tomoya Kitazaki, \\ Hanyue Kang, Satoru Adachi, Ichiro Ishimaru
}

Faculty of Engineering, Kagawa University, 2217-20 Hayashi, Takamatsu, Kagawa, 761-0396, Japan

E-mail: ishimaru@eng.kagawa-u.ac.jp; HP: http://www.eng.kagawa-u.ac.jp/ ishimaru/index.html

\begin{abstract}
We propose a machine learning method based on normalized correlation feature space for rapid adaptation to individual optical differences for mid-infrared spectroscopy applications. In the method, the number of feature variables was reduced to three parameters, comprising the correlation coefficient, the inclination and the $y$-intercept of the linear regression. Previously, convergences of the gradient method such as backpropagation were affected by noisy data. Therefore, stable spectral absorbances that were essentially derived from specific components such as glucose were extracted from measured spectral waveforms by eliminating these noisy waveforms. Therefore, we have successfully demonstrated the feasibility of the method for reference waveform extraction from actual noisy measured spectra by performing a cluster analysis of the normalized correlation feature space.
\end{abstract}

Keywords: noninvasive blood glucose sensor, mid-infrared spectroscopy, hyperspectral camera, machine learning

\section{Introduction}

Noninvasive blood glucose sensors are strongly required for daily healthcare monitoring applications. As illustrated in Figure 1, an ultrasonic-assisted mid-infrared spectroscopy technique for glucose sensors has been proposed previously [1]-[4]. A standing ultrasonic wave is generated and forms an internal reflection plane near the skin surface at a depth of $100 \mu \mathrm{m}$, for example. Therefore, mid-infrared light that is reflected from the internal biomembrane can be detected and converted into glucose concentration signals based on the spectral absorbance properties. However, to enable introduction of midinfrared spectroscopy into daily life environments, the sensor itself will require autonomous measurement capabilities to adapt for individual optical differences, such as the skin's color or moisture-retaining properties.

In addition, machine learning technologies such as deep learning have been strongly expected to increase the intelligence of various systems. However, in the deep learning case, astronomical amounts of teaching data were inevitably required to fill the hyper-multidimensional feature space fully. The effectiveness of deep reinforcement learning was verified by its ability to obtain abundant teaching data for Japanese chess and the game of Go such that computers themselves were able to judge which players were winning or losing. However, for healthcare sensor applications, it would be impossible to gather astronomical quantities of teaching data for a specific person.

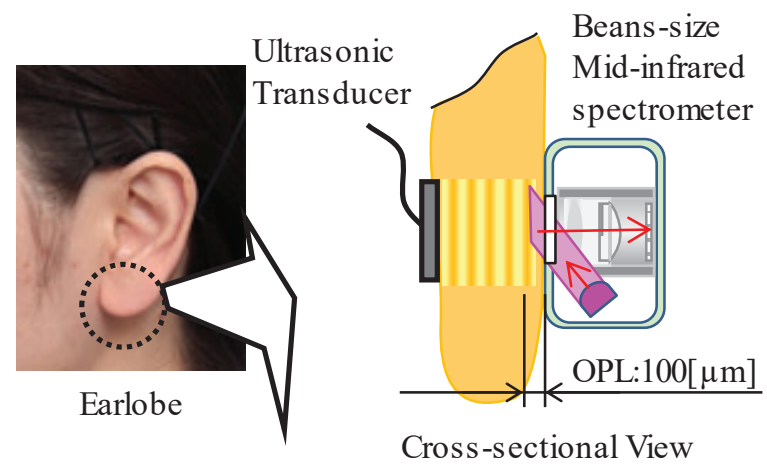

Figure 1. Ultrasonic-assisted mid-infrared spectroscopy for noninvasive blood glucose sensor [4].

Herein, we propose machine learning for rapid adaptation to the individual optical differences of spectroscopic characteristics. In this method, the narrow feature space is defined using three parameters (the correlation coefficient, the inclination and the $y$-intercept of the linear regression) based on a normalized correlation [5]. The convergence behavior of the gradient method was affected by noisy data. Therefore, stable spectral absorbance waveforms that were derived from essential 
components such as glucose were extracted from multiple measured spectra by eliminating these noisy waveforms. We therefore propose cluster analysis for reference waveform extraction from measured spectra in the normalized correlation feature space.

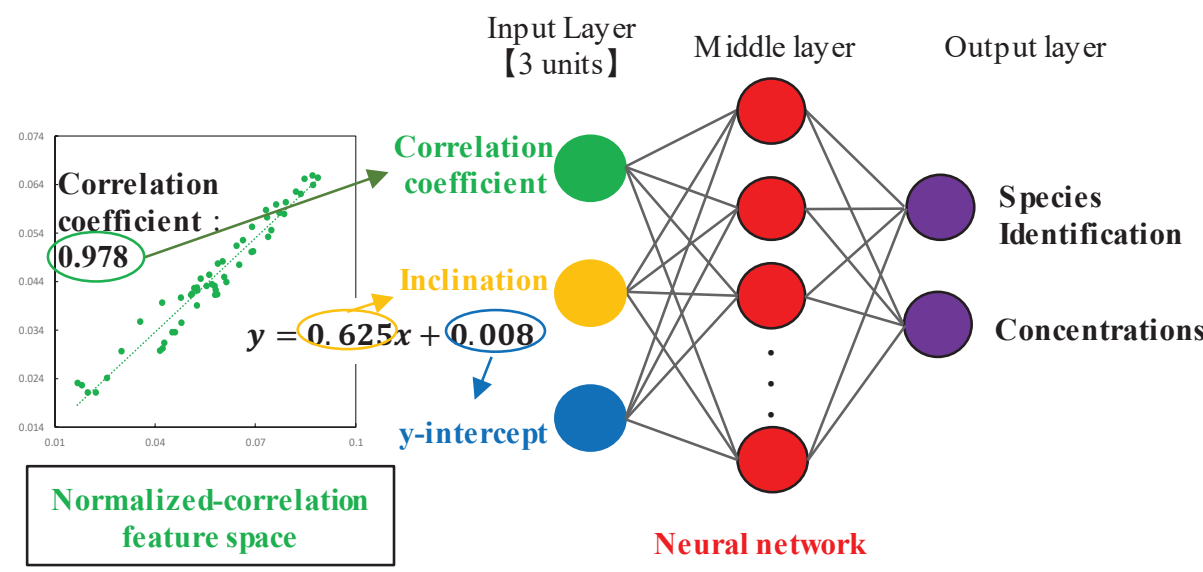

Figure 2. Schematic diagram of rapid machine learning scheme based on the normalized correlation method [5].

\section{Rapid machine learning based on the} normalized correlation feature space

1. Narrow feature space for smaller numbers of teaching data for machine learning [5]

As shown in Figure 2, to reduce the feature space dimensions, we select the correlation coefficient, the inclination and the $y$-intercept of the linear regression equation based on the normalized correlation method [5]. In the normalized correlation method, each of the absorbances in the reference waveform and the measured waveform are compared at the same wavelength. For example, at an arbitrary wavelength $\lambda$, the $x$ and $y$ coordinates are defined by the absorbances of the reference and measured waveforms at the same wavelength $\lambda$, respectively. If the reference waveform and the measured waveform have similar shapes, the correlation coefficient becomes close to 1 . In addition, the inclination of the linear regression equation indicates the height ratio of the absorbance peak that corresponds to the concentration of each component, while a $y$-intercept

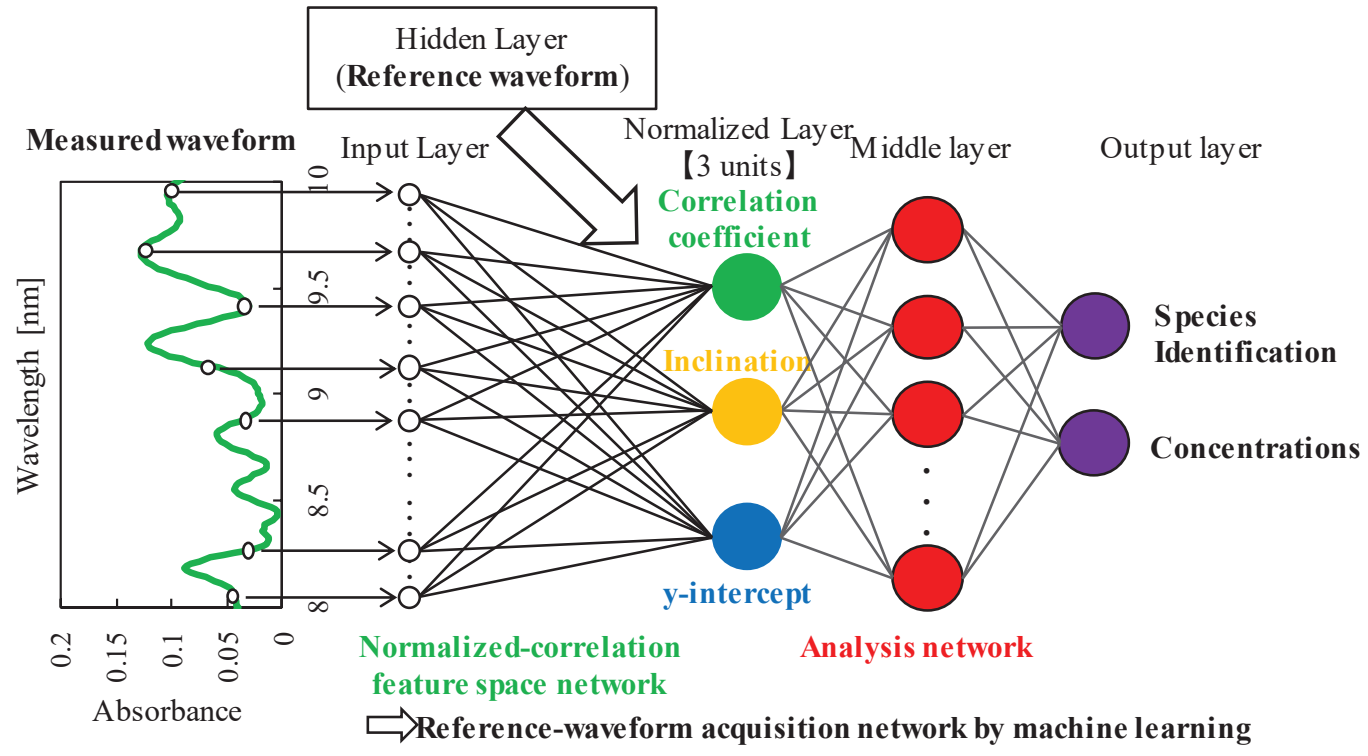

Figure 3. Schematic diagram for construction of the normalized correlation feature space from a reference waveform and a measured waveform in a neural network manner. 
indicates baseline differences that are caused by light source intensity fluctuations. Figure 3 shows a schematic diagram of the construction of the normalized correlation feature space in a neural network manner. We are trying to develop this network into a reference waveform acquisition network while eliminating noisy data using the backpropagation algorithm.

In the next section, we describe cluster analysis of the normalized correlation feature space for reference waveform extraction.
2. Cluster analysis for preliminary evaluation to extract reference waveforms from multiple measured teaching data in normalized correlation feature space by eliminating noisy data

To secure high convergence performance in conjugategradient machine learning methods such as backpropagation, elimination of noisy data is an effective approach to reduce the accumulation of evaluationfunction errors. Therefore, from the actual measured teaching data, it should be possible to extract the reference waveform without the noisy signals.

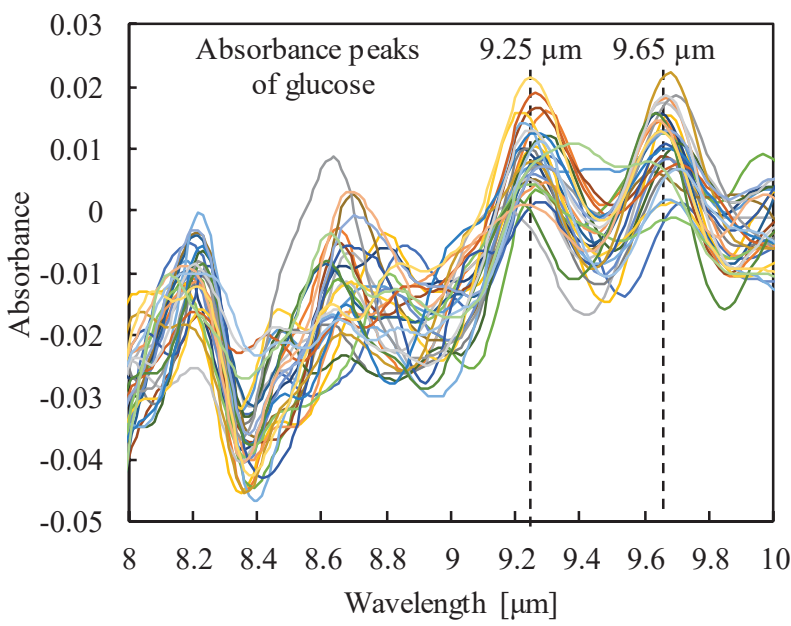

(b) Absorbance of glucose solutions (concentration: 100mg/dl)

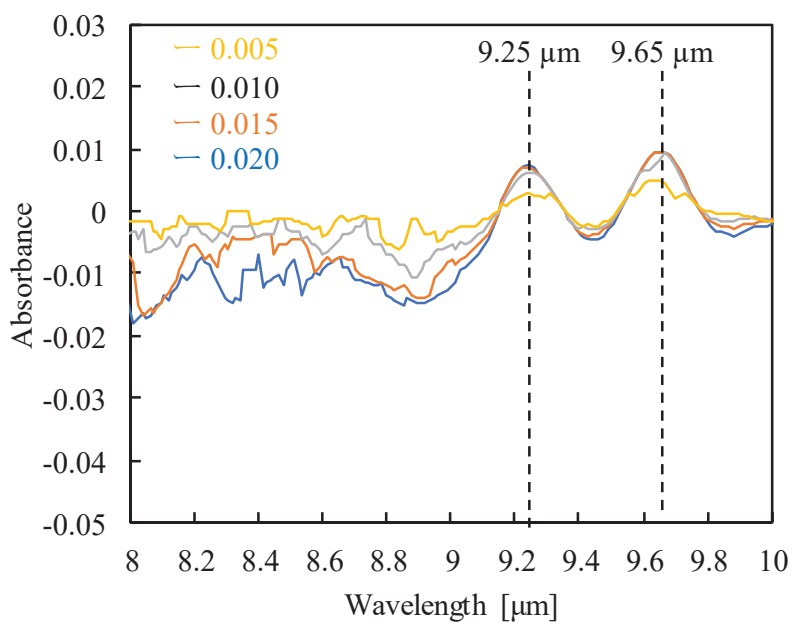

(d) Absorbance graph for each threshold norms

(c) The reference-waveform cluster by the least squares method

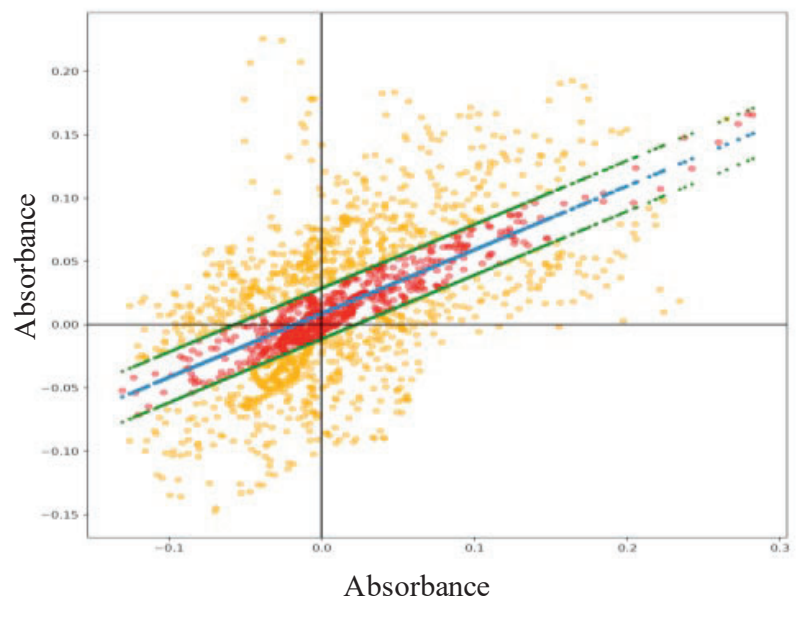

Figure 4. Demonstration of feasibility of extraction of static feature waveforms from an actual noisy measured spectrum by clustering in the normalized correlation feature space. 
As shown in Figure 4(a), we measured glucose solutions (concentration: $100 \mathrm{mg} / \mathrm{dl}$ ) 30 times using the proposed mid-infrared spectrometer (wavelength range: 8 $\mu \mathrm{m}-14 \mu \mathrm{m})[6]$. We calculated the correlation coefficients of every conceivable combination, as shown in Figure 4(b). In the cluster analysis, we defined the referencewaveform cluster using norms from a straight line that was calculated using the least squares method, as shown in Figure 4(c). In other words, some of the data located outside the cluster correspond to noise signals. Using only the data located inside the cluster, the reference waveforms were reconstructed as shown in Figure 4(d). We varied the threshold norms that define the cluster partition using values of $0.020,0.015,0.010$, and 0.005 . As shown in Figure 4(d), the specific absorbance peaks of glucose $(@ 9.25 \mu \mathrm{m}$ and $9.65 \mu \mathrm{m})$ are gradually clarified by reducing the threshold norms. Therefore, we have demonstrated the feasibility of extracting reference waveforms from an actual noisy measured spectrum by performing a cluster analysis in the normalized correlation feature space.

\section{Conclusions}

To allow a noninvasive blood glucose sensor based on mid-infrared spectroscopy to adapt to individual optical differences, a machine learning method that was defined in the normalized correlation feature space was proposed. We demonstrated the feasibility of extraction of the reference waveform from multiple noisy measured spectra by performing a cluster analysis in the normalized correlation feature space.

In future work, we intend to develop the normalized correlation feature space network into a reference waveform acquisition network using the backpropagation algorithm.

\section{References}

[1] Natsumi Kawashima; Kosuke Nogo; Satsuki Hosono; Akira Nishiyama; Kenji Wada; Ichiro Ishimaru, "Sensitivity improvement of one-shot Fourier spectroscopic imager for realization of noninvasive blood glucose sensors in smartphones", Opt. Eng. 55(11) 110506 doi: 10.1117/1.OE.55.11.110506 (2016)

[2] Ichiro ISHIMARU, Natsumi KAWASHIMA, Satsuki HOSONO, "Built-in hyperspectral camera for smartphone in visible, near-infrared and middle- infrared lights region (1st. report) - Trial products of beans-size Fourier-spectroscopic line-imager and feasibility experimental results of middle infrared spectroscopic imaging -", Proc. SPIE 9855, NextGeneration Spectroscopic Technologies IX, 985504 (2016)

[3] Natsumi KAWASHIMA, Satsuki HOSONO, Ichiro ISHIMARU, Built-in hyperspectral camera for smartphone in visible, near-infrared and middleinfrared lights region (2nd.report) - Sensitivity improvement of Fourier spectroscopic imaging to detect diffuse reflection lights from internal human tissues for healthcare sensors -, Proc. of SPIE Defense and Commercial Sensing Conference, DOI: 10.1364/3D.2018.JM4A.2 (2016)

[4] Tomoya KITAZAKI, Natsumi KAWASHIMA, Naoyuki YAMAMOTO, Hiroyuki NOMURA,Akira NISHIYAMA, Kenji WADA, Ichiro ISHIMARU,"Measurement of glucose concentrations inside agar using parametric standing wave to realize non-invasive blood glucose sensor", Optical Society of America Imaging and Applied Optics Congress DOI: 10.1364/3D.2018.JTu4A.18 (2018)

[5] Takashi AMAGAI, Ichiro ISHIMARU, "Feature Extraction from Infrared Spectroscopy by Machine Learning with Correlation coefficient as Error Function", Journal of Japanese Society for Artificial Intelligence, Vol.34, No.1 A (2019) in print

[6] Naoyuki Yamamoto,Natsumi Kawashima,Tomoya Kitazaki,Keita Mori,Hanyue Kang,Akira Nishiyama,Kenji Wada,Ichiro Ishimaru"Ultrasonic standing wave preparation of a liquid cell for glucosemeasurements in urine by midinfrared spectroscopy and potential application to smart toilets",Journal of Biomedical Optics pp. 050503-1 050503-4 [DOI: 10.1117/1.JBO.23.5.050503] Vol. 23(5) (2018) 


\title{
Rigorous analysis of reflection spectrum of absorbing film
}

\author{
Tetsuya Hoshino*a, Saswatee Banerjee ${ }^{\mathrm{a}}$, Norio Watanabe ${ }^{\mathrm{a}}$, Sadao Aoki ${ }^{\mathrm{a}}$, Kenji Sakurai ${ }^{\mathrm{b}}$ and \\ Masahide Itoh $^{\mathrm{a}}$
}

\author{
${ }^{a}$ Faculty of Pure and Applied Science, University of Tsukuba, 1-1-1 Tennoudai Tsukuba-shi, Ibaraki, \\ 305-8577, Japan \\ ${ }^{\mathrm{b} N a t i o n a l ~ I n s t i t u t e ~ f o r ~ M a t e r i a l s ~ S c i e n c e, ~ 1-2-1 ~ S e n g e n, ~ T s u k u b a, ~ I b a r a k i, ~ 305-0051, ~ J a p a n ~}$
}

\begin{abstract}
By analyzing both reflection and transmission spectra of an optical absorbing solid sample, reliable component identification of the material can be performed nondestructively. Since the reflection-absorption spectrum is affected by the surface shape of the film and the coherence of the light source, correction is to be made to calculate the extinction (or absorption) coefficient. Here, we used rigorous coupled-wave analysis (RCWA) that can evaluate the influence of polarized light and surface shape on diffraction angle distribution as a means of correction. Although RCWA is a coherent calculation, we developed a method of simulating incoherent light for absorption film. We determined the extinction coefficient of Rhodamine B film from the reflectance, which had the surface structure of wavelength order.
\end{abstract}

Keywords: absorption spectrum, coherence, film, surface structure

\section{Introduction}

Absorption spectra are widely used from soft X-rays to terahertz waves and are useful for identifying and quantifying unknown components. It is potentially superior in quantitative and nondestructive evaluation in comparison with the other types of spectroscopy. To make full use of its merit, reliable evaluation is important. If the extinction coefficient (proportional to the absorption coefficient) is as high as 0.1 or more in the resonance domain (about 1 to 20 wavelengths) of a particle or film, there will be little difference in the amount of transmitted light, which makes accurate measurement difficult. In that case, reflectance measurement is indispensable.

Since the reflectance is susceptible to the convex shape and the real part of the refractive index, correction is necessary. However, it was difficult to take these effects into consideration in the spectrum measurement of the unknown sample, because evaluation of size and shape of a convex was hindered by diffraction in the resonance domain. The geometrical optics does not evaluate the diffraction correctly. To resolve this problem, we developed the method to evaluate the extinction coefficient of particles of different shapes from diffraction pattern ${ }^{1}$. In this study, surface shape and the real part of the refractive index are considered in addition to the size and shape of the sample. As a case study, the film preparation, measurement and analysis were performed. Because the reflectivity is greatly influenced by the interference in the film, we also resolve this problem to evaluate the absorption correctly as shown below.

Here, RCWA was used as correction calculation. RCWA has the merit that it can directly evaluate the influence on the scattering angle distribution in the far field from polarized light and convex surfaces in the resonance domain on the surface ${ }^{2}$. On the other hand, incoherent light cannot be accurately evaluated because RCWA is coherent calculation. Conventionally, the result of incoherent light can be obtained by changing film thickness, and averaging the results ${ }^{3,4}$. They showed that the reflectivity of coherent light changed periodically against thickness and averaging the results gave us the incoherent reflectivity. However, in the presence of

** hoshino.tetsuya.gt@u.tsukuba.ac.jp; phone +81 29 853-5042 
absorption, the amount of light absorption varies with thickness, which makes this process difficult. We solved this problem by adding a transparent film to the absorbing layer and changing its film thickness. The thickness of the additional layer does not affect the absorption. By changing this thickness and averaging the reflectivity enable us to calculate the incoherent reflectivity. This calculation enables us to calculate the incoherent area and coherent area at the same time. We simulate the light control film for infrared cut glass by switching the incoherent area to coherent area as the example.

\section{Simulation}

\section{Method}

To simulate incoherent light's reflectivity by coherent simulation method RCWA, we added one clear layer 2 to absorptive layer 1 in Fig. 1. Here, $d_{1}$ and $d_{2}$ are thickness of each film, $n_{0}$ and $n_{1}$ are real part of refractive index and coefficient $k$ is the imaginary part of refractive index. $R_{0}$, $R_{1}$ and $R_{2}$ are the reflectivity of each boundary. Incident light enters normally the film from surface of layer 1 . To check the effect of the layer, we subtract $R_{1 l}$ (the reflectivity of layer 1 only) from $R_{2 l}$ (the reflectivity of the two layer) according to geometrical optics. Equations (1) to (4) are the results. Here, $\lambda$ is wavelength.

$$
\begin{aligned}
& T_{1}=\mathrm{e}^{-2 \gamma} . \\
& \gamma=2 \pi k d_{1} / \lambda . \\
& R_{2 l}-R_{1 l} \\
& =\frac{T_{1}^{4} R_{0}^{2}\left(1-2 R_{0}\right)\left(1-R_{1}-R_{2}+R_{1} R_{2}\right)+T_{1}^{2} X+R_{0} R_{1}\left(R_{0}-R_{2}\right)}{\left\{1-R_{1} R_{2}-T_{1}^{2} R_{0}\left(R_{1}+R_{2}-R_{1} R_{2}\right)\right\}\left(1-T_{1}^{2} R_{0}\right)}
\end{aligned}
$$

where

$$
\begin{aligned}
X= & R_{0}^{3}\left(R_{1} R_{2}-2 R_{1}-1\right)+R_{0}^{2}\left(-2 R_{1} R_{2}+2 R_{1}+R_{2}+2\right) \\
& +R_{0}\left(3 R_{1} R_{2}-2 R_{1}-2 R_{2}\right)-2 R_{1} R_{2}-R_{0}+R_{1}+R_{2}
\end{aligned}
$$

Eq. (3) and some simulation indicated that the following conditions were important for us to assume $R_{2 l}$ to be $R_{1 l}$.

$k>\lambda /\left(2 \pi d_{1}\right)$ or $k<0.01 \lambda /\left(2 \pi d_{1}\right)$.
From the simulation, we found the following. As shown in Eqs. (1) and (2), $T_{1}$ is dependent on coefficient $k$. Roughly, when Eq. (5) is satisfied, $\left(T_{1}\right)^{2}$ is almost 0 or 1 and $R_{2 l}$ becomes same to $R_{1 l}$ for most $R_{0}, R_{1}$ and $R_{2}$. We use this relationship to estimate the real part of refractive index $n$. For the sample of this time, when $k>0.2$ or $k<$ $0.002, R_{2 l}$ is almost same to $R_{1 l}$.

The sample is made of absorptive layer and transparent thick substrate. We inserted transparent additional layer into it in Fig. 2. The additional layer has same refractive index $n_{1}$ to the absorbing layer.

\section{Sample and Measurement Method}

Rhodamine B in ethanol was spin coated over the slide glass and etched by focused ion beam (FIB) (see Fig. 3). From SEM and scatterometry ${ }^{1}$, we know that the thickness of the Rhodamine B film is $0.65 \mu \mathrm{m}$. The measurement was performed at several ten $\mu \mathrm{m}$ from the center of the square hole fabricated by FIB. This distance's parameter is $s$.

Halogen lamp and $10 \mu \mathrm{m}$ pinhole is set left side of Fig.4, and beam spot area at the sample is about $120 \mu \mathrm{m}^{2}$. For the transmissive spectrum measurement, $\mathrm{CCD}$ is changed to optical fiber of spectrum analyzer.

\section{Results and Discussion}

From the transmissive spectrum of Fig. 6, we can estimate the number of digits of the extinction coefficient $k$. From pattern of the reflective spectrum of Fig. 5, we judged the surface of the film at " $120 \mu \mathrm{m}$ " to be smooth. From the reflective spectrum of the " $120 \mu \mathrm{m}$ ", we know the complex refractive index. As this case shows,

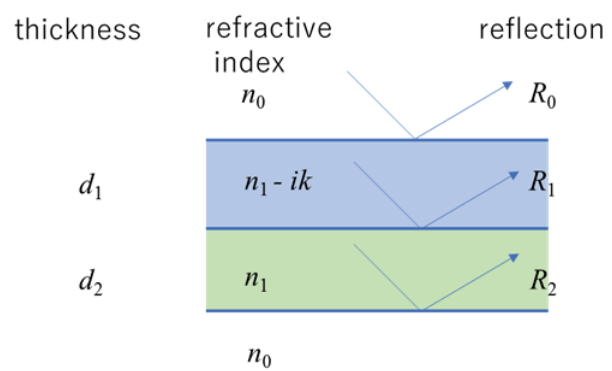

Figure 1. Simulation model for the single absorptive film. 


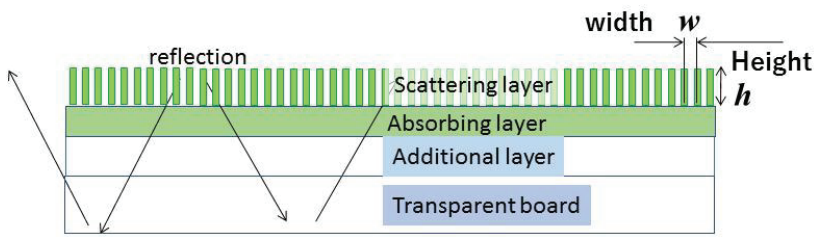

Figure 2. Simulation model for the absorptive film over transparent board in the experiment.

reflective spectrum is necessary to know not only the precise $k$ but also the refractive index ${ }^{5}$. From the reflective spectrum at the " $30 \mu \mathrm{m}$ " we know the surface structure. The crossing of the data curve at $0.54 \mu \mathrm{m}$ gave us the hint about the size of surface structure. In Fig. 6, the calculation conditions are $w=0.46 \mu \mathrm{m}$ and $h=$ $0.186 \mu \mathrm{m}$.

We use 1.5 for refractive index $n_{1}$ of the film for all wavelength at first. Next, we changed it so that simulation results should fit to the experimental data under the condition of Eq. (5). We use this refractive index to simulate $30 \mu \mathrm{m}$ and $120 \mu \mathrm{m}$ results. The complex refractive index of rough surface film can be estimated from Fig. 8. When extinction coefficient $k$ is more than 0.1 , transmissivity does not give correct $k$. We used the data of reflectivity also, and we were able to estimate $k$ more correctly.

From Figs. 5 and 6 , the $\lambda_{\max }$ ( $\lambda$ at the absorption maximum) is $580 \mathrm{~nm}$. Finally, we estimate $k$ at $\lambda_{\max }$ to be 0.27 from data of " $120 \mu \mathrm{m}$ " and 0.7 from data of " $30 \mu \mathrm{m}$ ". They roughly agree with conventional data ${ }^{6}$. Though the error seems to be large, this is much better than the case when the shape is unknown. The error can become as much as $1000 \%$. This is the clue to evaluation method of the complex refractive index of the particle whose shape and size is unknown.

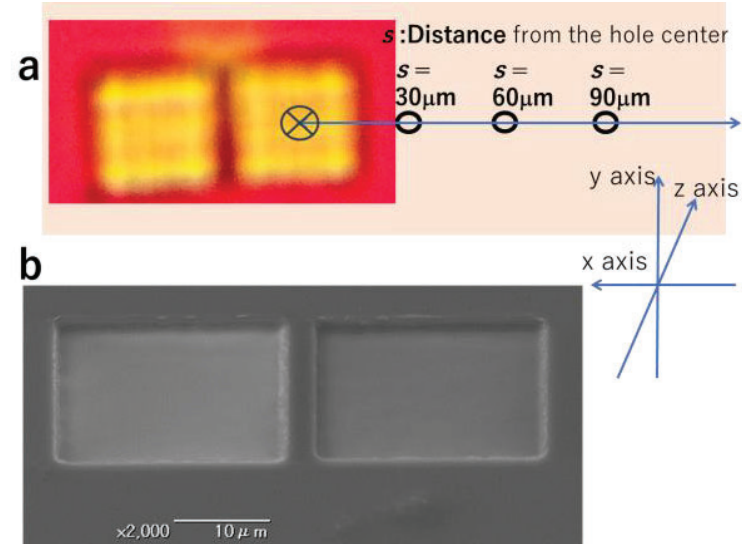

Figure 3. Rhodamine B membrane etched by FIB. a) Image of two square holes by optical micro scope and its coordinate. Three observed positions are indicated by circle. b) SEM image of the same part with $52^{\circ}$ slanted.

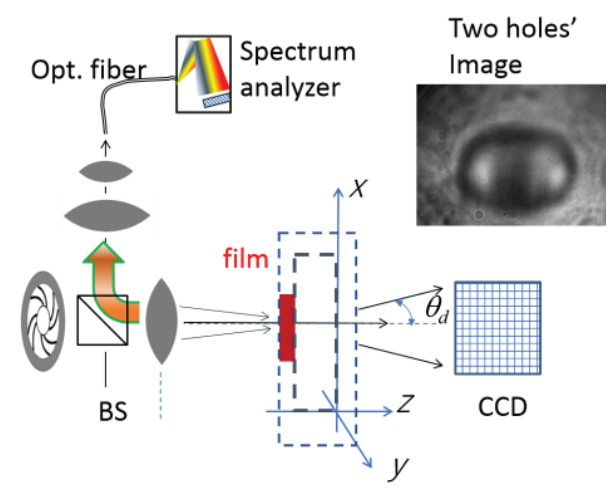

Figure 4. Reflective spectrum measurement system. Incident light passes through diaphragm, beam splitter, and lens. 


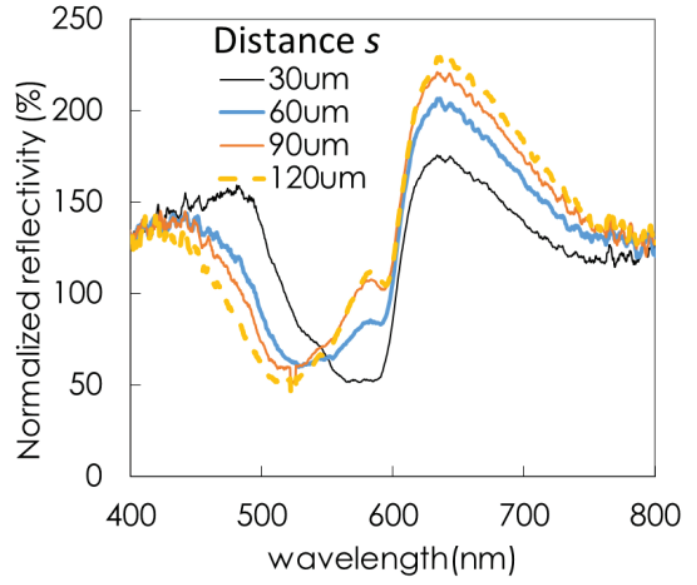

Figure 5. Reflective absorption spectrum of Rhodamine B film whose intensity is normalized by that of a slide glass.

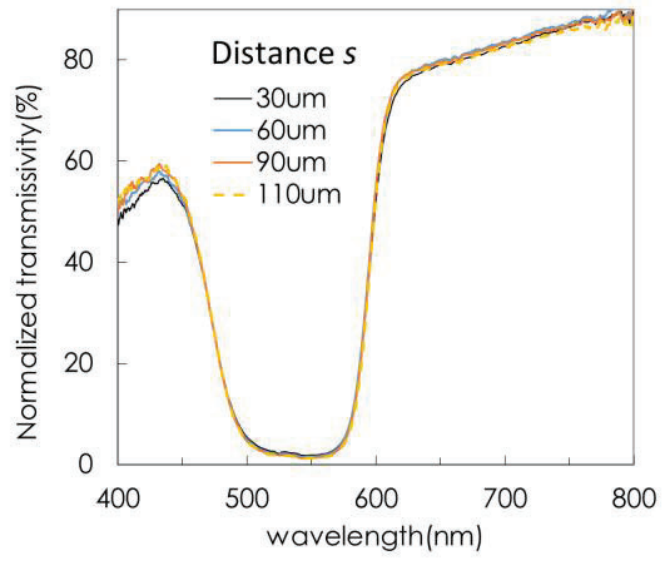

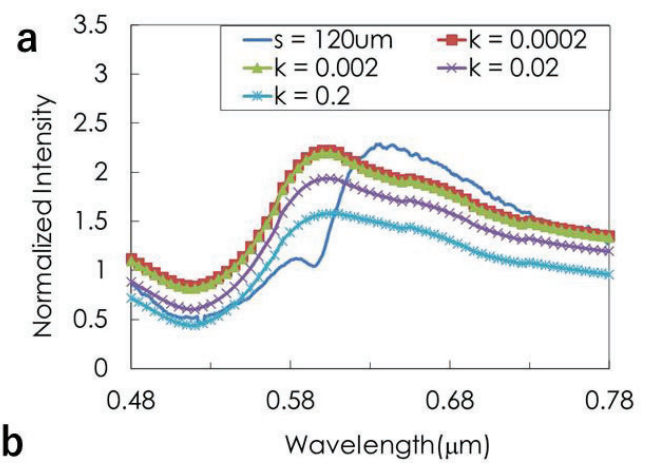

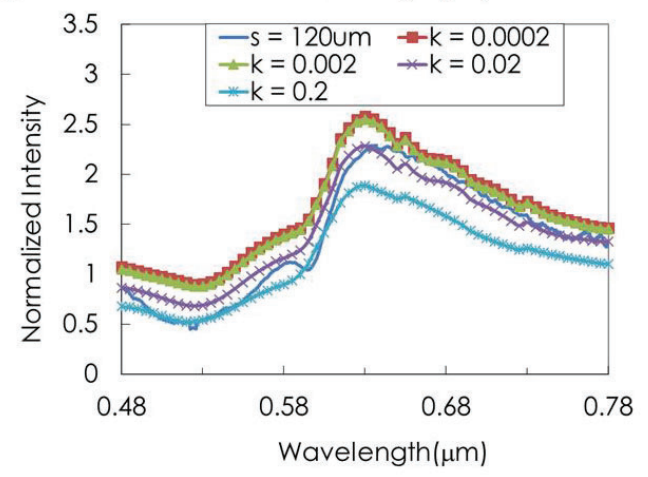

Figure 7. Calculation results and the experimental result of normalized reflective absorption spectrum. The observed data is " $120 \mu \mathrm{m}$ " away from the center of the hole. a) The extinction coefficient $k$ changes for every simulation. b) The wavelength dependence of the refractive index $n$ is considered.

Figure 6. Transmissive absorption spectrum whose intensity is normalized by that of a slide glass. 

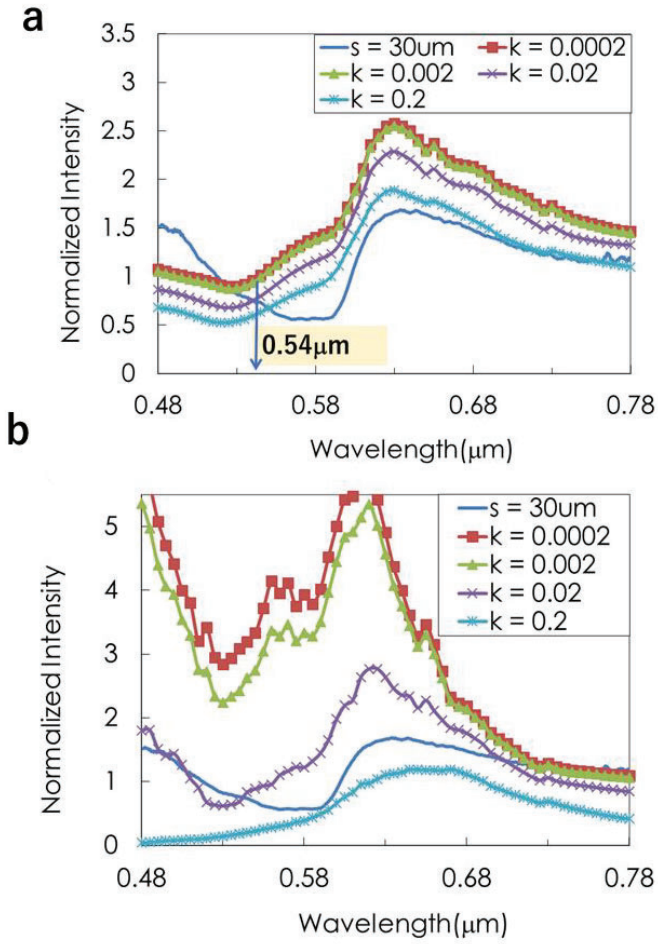

Figure 8. Calculation results and the experimental result. "s $=30 \mu \mathrm{m}$ " is the observed data. a) Simulation is same as that of Fig. 7b. b) The surface shape is considered in addition to the refractive index $n$.

\section{Summary}

The sample was found to be the film with rough surface with wavelength order. We non-destructively estimated complex refractive index of this unknown sample, considering its surface shape for the first time.

\section{Acknowledgement}

The manufacturing of the square holes was conducted at Advanced Characterization Nanotechnology Platform of the University of Tokyo.

\section{References}

[1] Hoshino, T., Watanabe, N., Aoki, S., Sakurai, K., and Itoh, M. "Cross-sectional particle measurement in the resonance domain on the substrate through scatterometry," Opt. Express 25(21), 26329-26348 (2017).
[2]Hoshino, T., Yatagai, T. and Itoh, M.. "Precise and rapid distance measurements by scatterometry," Opt. Express 20(4), 3954-3966 (2012).

[3] Campa, A., and Krc, J. "Two approaches for incoherent propagation of light in rigorous numerical

simulations," Progress In Electromagnetics Research, 137, 187-202 (2013).

[4] Puhan, J., Lipovšek, B., Bürmen, Á., and Fajfar, I. "An Accurate Representation of Incoherent Layers Within One-Dimensional Thin-Film Multilayer Structures With Equivalent Propagation Matrices," IEEE Photonics Journal, 9(5), 1-12 (2017).

[5] Banerjee, S., Hoshino, T., Cole, J.B., Aoki, S. and Itoh, M.," Computing refractive index of materials from thin film optical properties," OPTICS PHOTONICS JAPAN 2018 Extended Abstract, 30pBJ5, 87-88 (2018).

[6] Farag, A. A. M., and Yahia, I. S., "Structural, absorption and optical dispersion characteristics of rhodamine B thin films prepared by drop casting technique," Optics Communications 283(21), 4310-4317 (2010). 
The 1st Optical Wireless and Fiber Power Transmission Conference (OWPT2019), Yokohama, Japan, Apr. 23 - 25, 2019

\title{
Optical Design of Transmission Raman Spectrometer Based on the Plane Reflective Grating
}

\author{
Linlin Pei, Jianying Sun, Qunbo Lv
}

Academy of Opto-electronics, Chinese Academy of Sciences, No 9, Deng Zhuang South Road, HaiDian District, Beijing,100094, China

peill@aircas.ac.cn

\begin{abstract}
The Raman spectrometer can finely separate the optical signals. Different wavelengths of light are irradiated to different positions on the detector to obtain spectral information of the target. Improve the weak signal detection capability of the instrument and ensure the miniaturization of the system, in order to exert more application value of the system. We design a Transmission Raman Spectrometer Based on the Plane Reflective Grating. It provides important theoretical and technical guidance for the development of portable Raman spectrometers.
\end{abstract}

\section{Introduction}

Raman spectroscopy is a scattering spectrum of an object that reflects the molecular structure of a substance and provides rich information. It has played an important role in the fields of biology, chemistry, medical materials, food industry, and geological exploration. For the wider application of Raman spectroscopy, there is an urgent need for miniaturization of instruments ${ }^{[1]}$.

The widely-used Raman spectrometer system is the reflection structure of Czerny-Turner (C-T). This structure has no chromatic aberration and is not affected by the material transmittance. It is suitable for a wide spectral range. The transmission Raman spectroscopy system based on the plane reflection grating can better correct the system coma, realize the larger relative aperture, and the structure is simple and intuitive. This paper provides important theoretical and technical guidance for the development of portable Raman spectrometers.

\section{Optical system parameters}

The Raman spectrum range of commonly used materials is from 250 to $2875 \mathrm{~cm}^{-1}$, with $785 \mathrm{~nm}$ as the excitation wavelength, corresponding spectral range is from 797 $\mathrm{nm}$ to $1008 \mathrm{~nm}$. The general resolution of Raman spectral information is required to be $7 \sim 10.5 \mathrm{~cm}^{-1}$, that is, the spectral resolution is $0.6 \mathrm{~nm}$ at least in the whole band needs to be realized ${ }^{[2]}$. The smaller the grating constant, the smaller the slit, the higher the theoretical resolution of the spectrometer.

Table 1. Parameter of optical system

\begin{tabular}{c|c}
\hline \hline Parameter & Value \\
\hline Laser wavelength/nm & 785 \\
\hline Slit $/ \mu \mathrm{m}$ & 10 \\
\hline Wavelength range/nm & $797 \sim 1008$ \\
\hline Spectral resolution $/ \mathrm{nm}$ & 0.3 \\
\hline $\begin{array}{c}\text { Grating of groove } \\
\text { spacing/(lp/mm) }\end{array}$ & 1200 \\
\hline NA & 0.22 \\
\hline CCD pixel & $14 \mathrm{um}$ \\
\hline \hline
\end{tabular}

system which can achieve $0.3 \mathrm{~nm}$ spectral resolution in the full-band. The specific optical system design index parameters are shown in Table 1.

\section{Optical System Design}

The slit width is 10 microns, the angle of the incident grating is $15^{\circ}$, and the focal length of the collimating lens $\mathrm{f}_{1}^{\prime}$ satisfies the formula:

$$
\mathrm{f}_{1}^{\prime}=\frac{\mathrm{ad}}{\Delta \lambda \mathrm{m}} \cos \theta=27 \mathrm{~mm}
$$

Where a is the slit width, $d$ is the grating constant, $\Delta \lambda$ is the spectral resolution, and $\mathrm{m}$ is the diffraction order, $\theta$ is the grating incident angle ${ }^{[3]}$.

The collimating lens belongs to the conventional focal length and general relative aperture optical system. We can use a pair of double-glued lens to correct the aberration of $800 \sim 1008 \mathrm{~nm}$ wavelength, and collimate the output.

According to the grating equation:

$$
\mathrm{d}(\sin \theta+\sin \alpha)=\mathrm{m} \lambda
$$
$55.2^{\circ}$

where $\alpha$ is the grating exit angle. We can get $\alpha=$

Calculate the focal length of the imaging lens:

$$
\mathrm{f}_{2}^{\prime}=\frac{\mathrm{pd}}{\Delta \lambda \mathrm{m}} \cos \alpha=22.2 \mathrm{~mm}
$$

Where $\mathrm{p}$ is the pixel size.

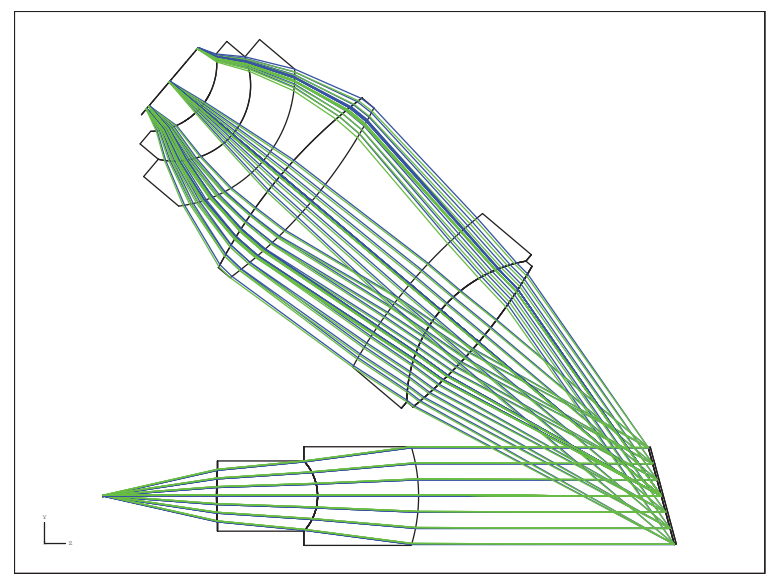

In this paper, we design a Raman Pspe.cof \&rRtertol. 11142 1114201-68 Fig.1. Structure of the system 
The 1st Optical Wireless and Fiber Power Transmission Conference (OWPT2019), Yokohama, Japan, Apr. 23 - 25, 2019 Fig.1.

The final optimized design optical system is shown in

It can be seen from the basic parameters that the spectroscope imaging lens belongs to the conventional focal length. Since the beam entering the imaging lens is increased after the beam is diffracted by the grating, a large relative aperture imaging lens is required to concentrate the beam.

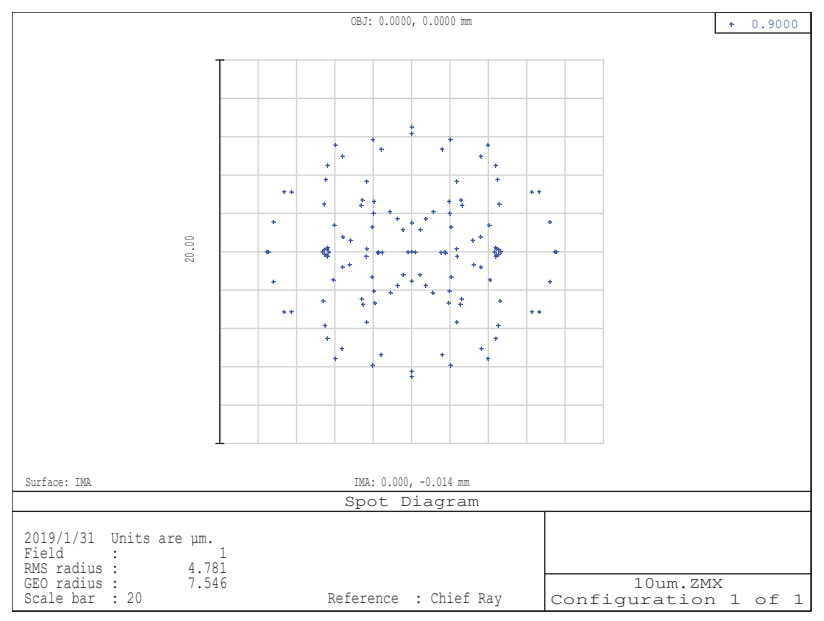

Fig.2. RMS of typical wavelength

The optical system image quality is evaluated by the RMS. It can be seen from the Fig.2, that the RMS of the typical wavelength is concentrated within one pixel.

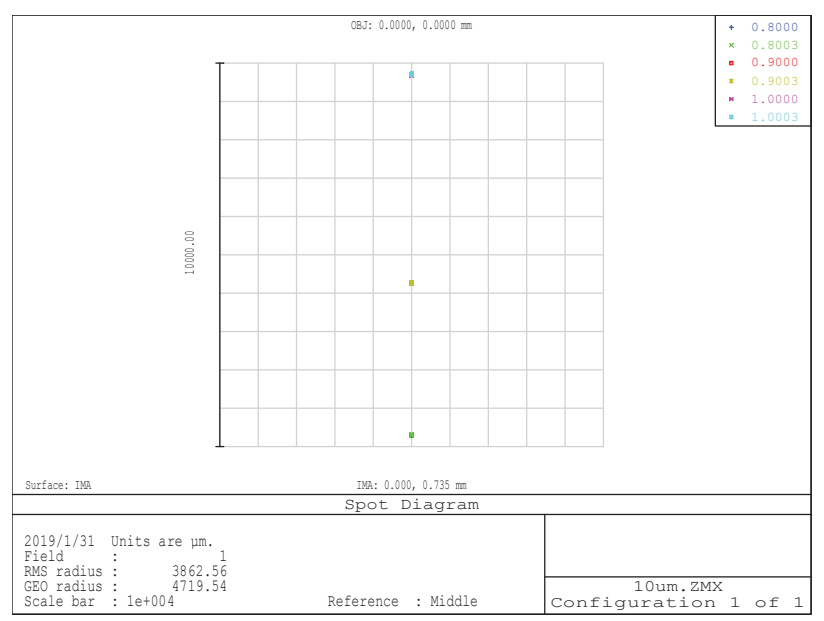

Fig.3. RMS of the full-band

The RMS of full-band is shown in Fig.3, it can be seen that the optical system has uniform dispersion.

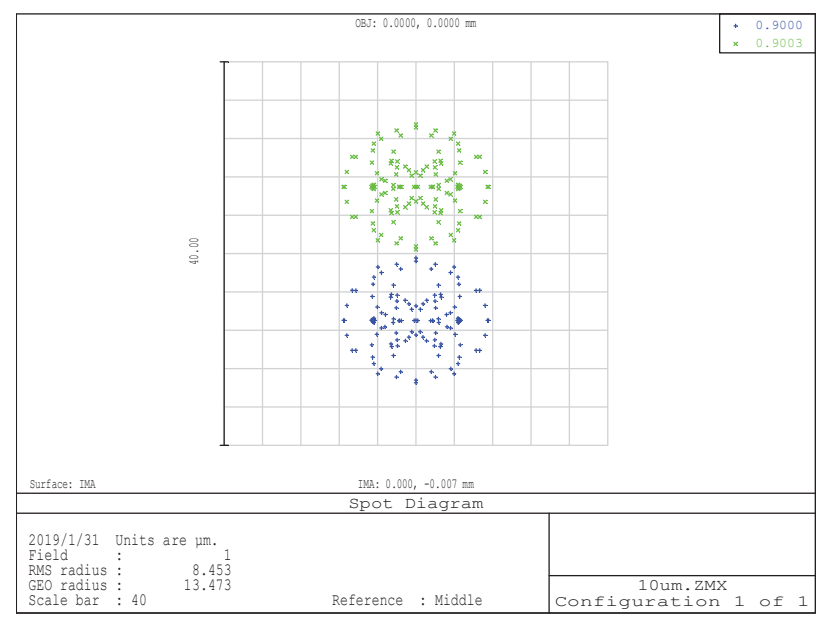

Fig.4. RMS of two typical wavelengths

Fig.4 is the RMS of two typical wavelengths, $900 \mathrm{~nm}$ and $900.3 \mathrm{~nm}$. It can be seen from the Fig.4 that two wavelength information can be separated independently.

The system has a total length of $60 \mathrm{~mm}$, a width of $60 \mathrm{~mm}$ and a thickness of $30 \mathrm{~mm}$. Small size and high resolution.

\section{Conclusion}

This paper mainly studies the spectrometer part of the Raman spectrometer system. The whole system is compact in structure, which can realize large relative aperture and compensate the low diffraction efficiency of the grating. Compared to the structural form of $\mathrm{C}-\mathrm{T}$, the transmission system is better able to concentrate the light within one pixel.

\section{Acknowledgment}

Project supported by the National Natural Science Foundation of China (Grant No. 61635002).

\section{References}

[1]. Liu Yande, Ye Bing. Quantitative Analysis of Omethoate Content Based on Raman Spectroscopy[J]. Chinese Journal of Agricultural Mechanization, 2014, 35(1): 88-91.

[2]. An Yan, Liu Ying, Sun Qiang, et al. Design and Development of Optical System for Portable Raman Spectrometer[J]. Acta Optica Sinica, 2013, 33(3): 300-306.

[3]. He Zhenlei. Design of optical system for Raman spectrometer for medicinal adult adulteration detection [D]. 2015. 


\title{
Polarization camera performance optimization
}

\author{
Nathan Hagen, Shibata Shuhei, and Yukitoshi Otani \\ Utsunomiya University, Department of Optical Engineering, Center for Optical Research and Engineering (CORE), Utsunomiya, Japan
}

Abstract. We outline a simple calibration method for microgrid polarization cameras that is easier than existing methods and apply the method to a commercial cameras. Experiments show the distribution of diattenuation and orientation over all of the pixels of the camera. Since the diattenuation values are low, and the orientation varies from pixel-to-pixel, we also outline a method for taking the calibration parameters and estimating the input polarization state. The resulting "optimization" method allows one to convert a low-performing camera into a high-performing one, albeit at lower SNR. Keywords: Polarization, Imaging polarimetry, Image processing

\section{Introduction}

Polarization cameras use an array of micropolarizer filters aligned to the detector array pixels, with the micropolarizers oriented at angles $0^{\circ}, 45^{\circ}, 90^{\circ}$, and $135^{\circ}$. Due to difficulty of manufacture, micropolarizer filters have had trouble achieving high quality measurements with good orientation accuracy and high polarization extinction ratio. We show a simple method for calibrating the polarization properties of each micrpolarizer pixel and show how to compensate for non-ideal characteristics in order to optimize measurements.

Each micropolarizer can be modelled as an oriented linear diattenuator, having mean transmission $A$, diattenuation $D$, and orientation angle $\alpha$, represented by the Mueller matrix

$$
\begin{aligned}
& \mathbf{M}_{\mathrm{ld}}(A, D, \alpha)= \\
& A\left(\begin{array}{cccc}
1 & D \cos (2 \alpha) & D \sin (2 \alpha) & 0 \\
D \cos (2 \alpha) & 1-D \sin ^{2}(2 \alpha) & \frac{1}{4 A} D \sin (4 \alpha) & 0 \\
D \sin (2 \alpha) & \frac{1}{4 A} D \sin (4 \alpha) & 1-D \cos ^{2}(2 \alpha) & 0 \\
0 & 0 & 0 & 1-D
\end{array}\right)
\end{aligned}
$$

In order to measure the orientation and diattenuation of each pixel in the polarization camera, we use the system layout shown in Fig. 1. Due to the separation distance, the filtered white light source is nearly collimated when it passes through a linear polarizer oriented at angle $\theta$. This polarizer is the "generator" that we use for calibration. For unpolarized light $\mathbf{s}_{\text {in }}=\left(I_{0}, 0,0,0\right)^{\top}$ incident on the generating polarizer, light passes through each pixel's microdiattenuator and reaches the detection layer, so that the Mueller matrix measurement model for the optical signal is

$$
g=\mathbf{d} \cdot \mathbf{M}_{\mathrm{ld}}(q, r, \alpha) \cdot \mathbf{M}_{\mathrm{lp}}(\theta) \cdot \mathbf{s}_{\mathrm{in}} .
$$

for input Stokes vector $\mathbf{S}_{\mathrm{in}}$ and detection row vector $\mathbf{d}=(\eta, 0,0,0)$ for quantum efficiency $\eta$. Here the detected signal $g$ is in units of photoelectrons.

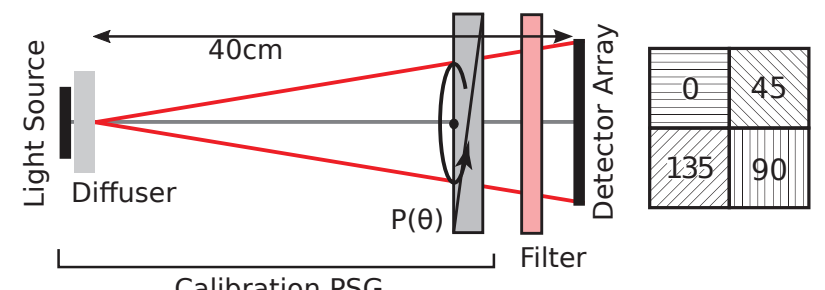

Fig 1: Optical layout for polarization camera calibration. The optical filter is a $532 \pm 10 \mathrm{~nm}$ narrowband spectral filter.
Setting the generating polarizer to orientations $\theta=0^{\circ}, 45^{\circ}$, $90^{\circ}$, and $135^{\circ}$, we obtain four measurements

$$
\begin{aligned}
g_{0} & =I_{\mathrm{e}}[1+D \cos (2 \alpha)]+n_{0} \\
g_{45} & =I_{\mathrm{e}}[1+D \sin (2 \alpha)]+n_{45} \\
g_{90} & =I_{\mathrm{e}}[1-D \cos (2 \alpha)]+n_{90} \\
g_{135} & =I_{\mathrm{e}}[1-D \sin (2 \alpha)]+n_{135}
\end{aligned}
$$

Whereas previous calibration methods fitted pixel diattenuation parameters using images taken at a large number of different input polarization angles, ${ }^{1-3}$ the above choice of four angles produces simple formulas for estimating the incident intensity $I_{\mathrm{e}}$ and the polarization properties at each pixel $\alpha, D$, and $X$ :

$$
\begin{aligned}
\hat{I}_{\mathrm{e}} & =\frac{1}{4}\left(g_{0}+g_{45}+g_{90}+g_{135}\right) \\
\hat{\alpha} & =\frac{1}{2} \arctan \left[\left(g_{0}-g_{90}\right) /\left(g_{45}-g_{135}\right)\right] \\
\hat{D} & =\frac{2\left[\left(g_{0}-g_{90}\right)^{2}+\left(g_{45}-g_{135}\right)^{2}\right]^{1 / 2}}{g_{0}+g_{45}+g_{90}+g_{135}} \\
\hat{X} & =(1+\hat{D}) /(1-\hat{D})
\end{aligned}
$$

Because the estimates do not depend on the intensity $I_{\mathrm{e}}$ at each pixel, calibrating the camera does not require spatially uniform illumination. While differences in light level at the camera will produce differences in noise at each pixel, this broadening can be made small in comparison to the differences produced by manufacturing variations.

\section{Experimental results}

Using the above calibration method, we measured the diattenuation parameters of a commercial polarization camera, with a $1200 \times 1800$ grid of $7.4 \mu \mathrm{m}$ pixels, $110 \mathrm{~Hz}$ frame rate, and $12 \mathrm{bit}$ depth. Figure 2 shows the histograms of the calibrated pixel characteristics. Each parameter is calculated separately for each of the four different pixel types $\left(0^{\circ}, 45^{\circ}, 90^{\circ}\right.$, and $\left.135^{\circ}\right)$. The optical system axis is defined such that the mean of the $0^{\circ}$ pixels is exactly zero.

The experimental setup uses a linearly polarized incoherent light source, generated using a white light LED, diffuser, rotatable linear polarizer (Glan-Thompson type), and a $532 \pm 10 \mathrm{~nm}$ narrowband spectral filter. The initial datasets were collected by setting the generating polarizer to $0^{\circ}$ and summing over 100 frames in order to reduce the measurement noise. The same procedure was then used for the $45^{\circ}, 90^{\circ}$, and $135^{\circ}$ orientations of the generating polarizer, and for the estimation of the background. After 
subtracting the background, the summed images were scaled from digital counts to photoelectron units using the camera's gain, 8.0 photoelectrons per digital count. Taking the sum of the images, rather than mean as is more common, is done in order to maintain Poisson statistics in the data.

Unlike previous calibration methods, we do not use an integrating sphere in order to make the illumination field uniform, as exact uniformity is unnecessary for the parameter estimates. The main purpose of the diffuser in the experimental setup is to remove any residual polarization from the light source.

\section{Compensating for non-ideal performance}

If we model each pixel of a polarization camera as a nonideal polarization element, then we have a diattenuation and orientation parameter that we need to calibrate for each one. Using Mueller calculus for a linear diattenuator element with diattenuation $D_{n}$ and oriented at angle $\alpha_{n}$, we obtain the measurement model

$$
\underbrace{\left(\begin{array}{l}
g_{0} \\
g_{1} \\
g_{2} \\
g_{3}
\end{array}\right)}_{\mathbf{g}}=\frac{1}{2} \underbrace{\left(\begin{array}{lll}
2 & D_{0} \cos \left(2 \alpha_{0}\right) & D_{0} \sin \left(2 \alpha_{0}\right) \\
2 & D_{1} \cos \left(2 \alpha_{1}\right) & D_{1} \sin \left(2 \alpha_{1}\right) \\
2 & D_{2} \cos \left(2 \alpha_{2}\right) & D_{2} \sin \left(2 \alpha_{2}\right) \\
2 & D_{3} \cos \left(2 \alpha_{3}\right) & D_{3} \sin \left(2 \alpha_{3}\right)
\end{array}\right)}_{\mathbf{M}} \underbrace{\left(\begin{array}{c}
s_{0} \\
s_{1} \\
s_{2} \\
s_{3}
\end{array}\right)}_{\mathbf{S}},
$$

where the $g_{n}$ are the measurements from four adjacent pixels (nominally oriented to $0^{\circ}, 45^{\circ}, 90^{\circ}$, and $135^{\circ}$ ), $\mathbf{M}$ is the measurement matrix, and $\mathbf{s}$ is the Stokes vector of the input light. If we try to invert this measurement system as-is, we get a mess. However, if we assume that the orientation errors are small, then we can use the small angle approximation, so that all of the cosine terms are set to 1 ,

$$
\begin{array}{ll}
\cos \left(2 \alpha_{0}\right) \rightarrow 1, & \cos \left(2 \alpha_{1}\right) \rightarrow-2 \epsilon_{1}, \\
\cos \left(2 \alpha_{2}\right) \rightarrow-1, & \cos \left(2 \alpha_{3}\right) \rightarrow 2 \epsilon_{3},
\end{array}
$$

and all of the sine terms can be set to their linear arguments about the nominal angle,

$$
\sin \left(2 \alpha_{0}\right) \rightarrow 2 \epsilon_{0}, \quad \sin \left(2 \alpha_{1}\right) \rightarrow 1,
$$

$$
\sin \left(2 \alpha_{2}\right) \rightarrow-2 \epsilon_{2}, \quad \sin \left(2 \alpha_{3}\right) \rightarrow-1 .
$$

where $\epsilon_{n}$ represents the angular error about the nominal orientation. If we perform the pseudoinverse, we get the analysis matrix $\mathbf{A}=\mathbf{W}^{+}$used to estimate the input Stokes vector from the four intensity measurements:

$$
\underbrace{\left(\begin{array}{c}
\hat{s}_{0} \\
\hat{s}_{1} \\
\hat{s}_{2}
\end{array}\right)}_{\hat{\mathbf{s}}}=\underbrace{\left(\begin{array}{llll}
A_{00} & A_{01} & A_{02} & A_{03} \\
A_{10} & A_{11} & A_{12} & A_{13} \\
A_{20} & A_{21} & A_{22} & A_{23}
\end{array}\right)}_{\mathbf{A}} \underbrace{\left(\begin{array}{c}
I_{0} \\
I_{1} \\
I_{2} \\
I_{3}
\end{array}\right)}_{\mathbf{g}} .
$$

If we first consider the simpler case where the orientation errors are negligible $\left(\epsilon_{i}=0\right)$ then we get

$$
\begin{aligned}
& A_{00}=\frac{1}{2} B D_{2}\left(D_{0}+D_{2}\right)\left(D_{1}^{2}+D_{3}^{2}\right) \\
& A_{01}=\frac{1}{2} B\left(D_{0}^{2}+D_{2}^{2}\right) D_{3}\left(D_{1}+D_{3}\right) \\
& A_{02}=\frac{1}{2} B D_{0}\left(D_{0}+D_{2}\right)\left(D_{1}^{2}+D_{3}^{2}\right) \\
& A_{03}=\frac{1}{2} B D_{1}\left(D_{0}^{2}+D_{2}^{2}\right)\left(D_{1}+D_{3}\right) \\
& A_{10}=B\left[D_{2}\left(D_{1}^{2}+D_{3}^{2}\right)+2 D_{0}\left(D_{1}^{2}+D_{3} D_{1}+D_{3}^{2}\right)\right] \\
& A_{11}=-B\left[\left(D_{0}-D_{2}\right) D_{3}\left(D_{1}+D_{3}\right)\right] \\
& A_{12}=-B\left[D_{0}\left(D_{1}^{2}+D_{3}^{2}\right)+2 D_{2}\left(D_{1}^{2}+D_{3} D_{1}+D_{3}^{2}\right)\right] \\
& A_{13}=-B\left[D_{1}\left(D_{0}-D_{2}\right)\left(D_{1}+D_{3}\right)\right] \\
& A_{20}=-B\left[D_{2}\left(D_{0}+D_{2}\right)\left(D_{1}-D_{3}\right)\right] \\
& A_{21}=B\left[\left(2 D_{1}+D_{3}\right) D_{0}^{2}+2 D_{1} D_{2} D_{0}+D_{2}^{2}\left(2 D_{1}+D_{3}\right)\right] \\
& A_{22}=-B\left[D_{0}\left(D_{0}+D_{2}\right)\left(D_{1}-D_{3}\right)\right] \\
& A_{23}=-B\left[\left(D_{1}+2 D_{3}\right) D_{0}^{2}+2 D_{2} D_{3} D_{0}+D_{2}^{2}\left(D_{1}+2 D_{3}\right)\right]
\end{aligned}
$$

for

$$
\begin{gathered}
B^{-1}=D_{0}^{2}\left(D_{1}^{2}+D_{1} D_{3}+D_{3}^{2}\right)+D_{0}\left(D_{1}^{2} D_{2}+D_{2} D_{3}^{2}\right) \\
+D_{1}^{2} D_{2}^{2}+D_{1} D_{2}^{2} D_{3}+D_{2}^{2} D_{3}^{2}
\end{gathered}
$$

It is also possible to form the analysis matrix for the more general case $\left(\epsilon_{i} \neq 0\right)$, though the expressions become cumbersome.

\section{References}

1. T. York and V. Gruev, "Characterization of a visible spectrum division-of-focal-plane polarimeter," Appl. Opt. 51, 5392-5400 (2012).

2. S. B. Powell and V. Gruev, "Calibration methods for division-of-focal-plane polarimeters," Opt. Express 21, 21039-21055 (2013).

3. H. Fei, F.-M. Li, W.-C. Chen, R. Zhang, and C.-S. Chen, "Calibration method for division of focal plane polarimeters," Appl. Opt. 57, 4992-4996 (2018).

4. Y. Maruyama, T. Terada, T. Yamazaki, Y. Uesaka, M. Nakamura, Y. Matoba, K. Komori, Y. Ohba, S. Arakawa, Y. Hirasawa, Y. Kondo, J. Murayama, K. Akiyama, Y. Oike, S. Sato, and T. Ezaki, "3.2-MP back-illuminated polarization image sensor with fourdirectional air-gap wire grid and 2.5- $\mu$ m pixels," IEEE Trans. Elect. Dev. 65, 2544-2551 (2018). 

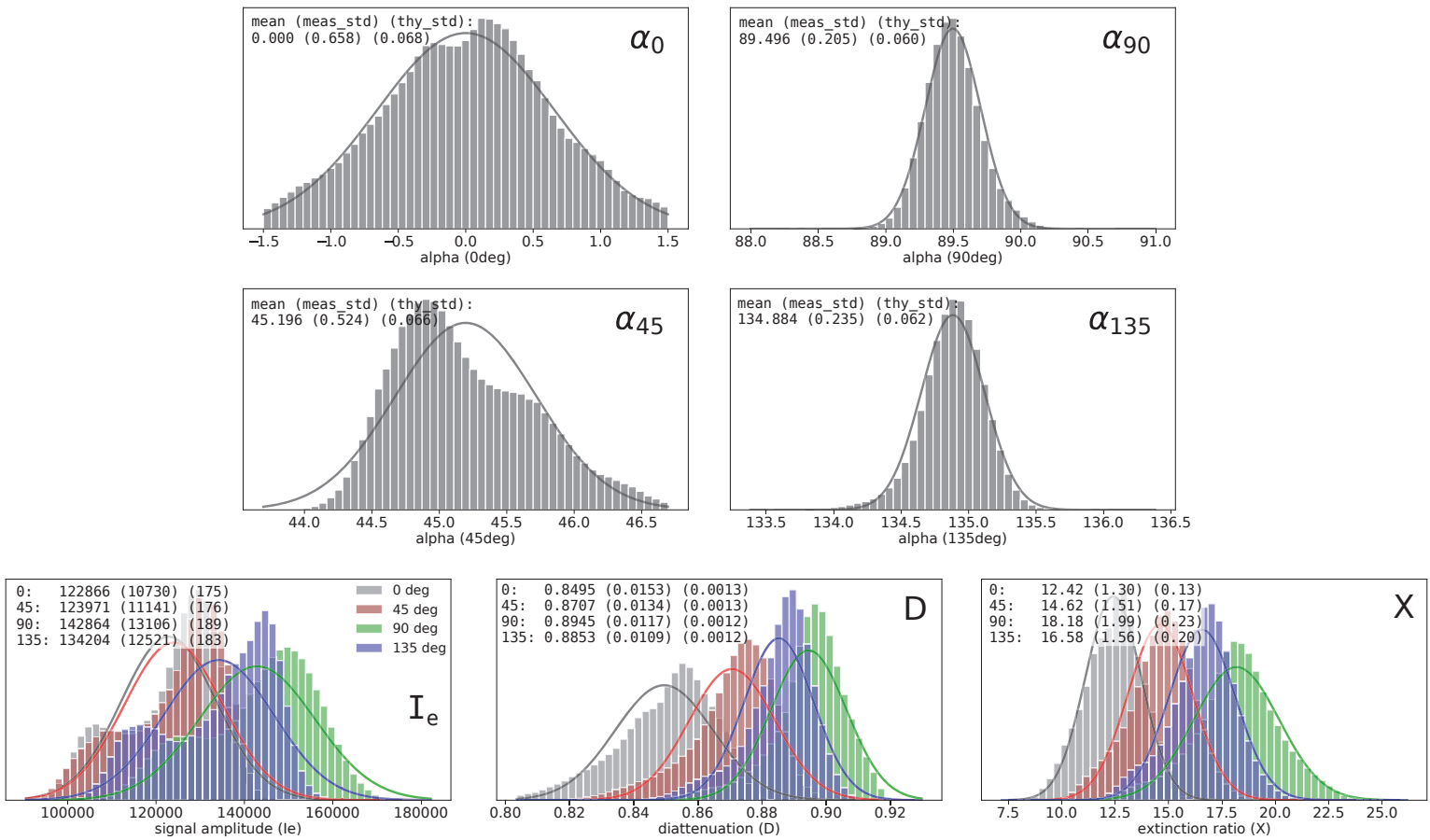

Fig 2: Calibration results for a polarization camera, showing the pixel histograms for the four orientation angles $\alpha_{0}$ through $\alpha_{135}$ and the three intensity/diattenuation parameters $I_{\mathrm{e}}, D$, and $X$. the detected intensity $I_{\mathrm{e}}$ is given in units of photoelectrons. The overlying curves are the equivalent Gaussian distributions, sharing the measured means and standard deviations. 


\title{
Snapshot imaging polarimetry based on structured interference fringes
}

\author{
Daesuk Kim* and Vamara Dembele \\ Division of Mechanical System Engineering, Chonbuk National University, 567 Baekje-daero, Jeonju, \\ 54896, Republic of Korea
}

\begin{abstract}
This paper describes a highly robust snapshot imaging polarimetry based on structured interference fringes. The proposed new concept on snapshot imaging polarimetry can provide dynamic measurement capability with high robustness and design flexibility. We demonstrate the proposed simple scheme can provide a spatially resolved polarimetric phase map $\Delta(x, y)$ in tens of milliseconds with high precision.
\end{abstract}

Keywords: imaging polarimetry, snapshot, structured fringe, phase extraction

\section{Introduction}

Polarization imaging is one of the most sensitive and versatile imaging tools utilized in various fields such as remote sensing, material property investigation, and biomedical imaging [1-3]. Conventional polarization imaging systems typically require either a mechanicallyrotated polarization modulation or highly-complicated and high cost electrical modulation devices to extract polarimetric imaging parameters. Since such conventional polarization imaging schemes based on multiple image acquisitions have inherent shortcomings in measurement speed although they can provide high precision, several new attempts have been made to overcome the speed limitation for achieving real time polarization imaging capability [4-8]. This paper describes a highly robust snapshot polarimetric phase imaging based on structured interference fringes. It can extract a polarimetric phase image map $\Delta(x, y)$ representing a spatially-resolved phase difference image between $\mathrm{p}$ - and s-polarizaiton of a measured object by using only a single polarization-modulated off-axis interferogram. Some experiments are conducted to verify the feasibility of the proposed concept by extracting spatially resolved polarization state variations generated by a QWP (Quarter Wave Plate). It can provide spatially resolved polarimetric phase image information in tens of milliseconds with high precision of a few degrees.

*dashi.kim@jbnu.ac.kr; phone 82-63-270-4632

\section{Results and Discussion}

To demonstrate the spatial polarimetric phase difference $\Delta(\mathrm{x}, \mathrm{y})$ measurement capability of the proposed snapshot imaging polarimeter system, we have conducted experiments by imaging the surface of the QWP by varying the angle of the QWP from $-45^{\circ}$ with an interval of $10^{\circ}$. Figure 1 illustrates the measured $\Delta(\mathrm{x}, \mathrm{y})$ obtained by varying the achromatic QWP rotation angle from $\theta=-$ $55^{\circ}$ to $-135^{\circ}$ with an interval of $10^{\circ}$.
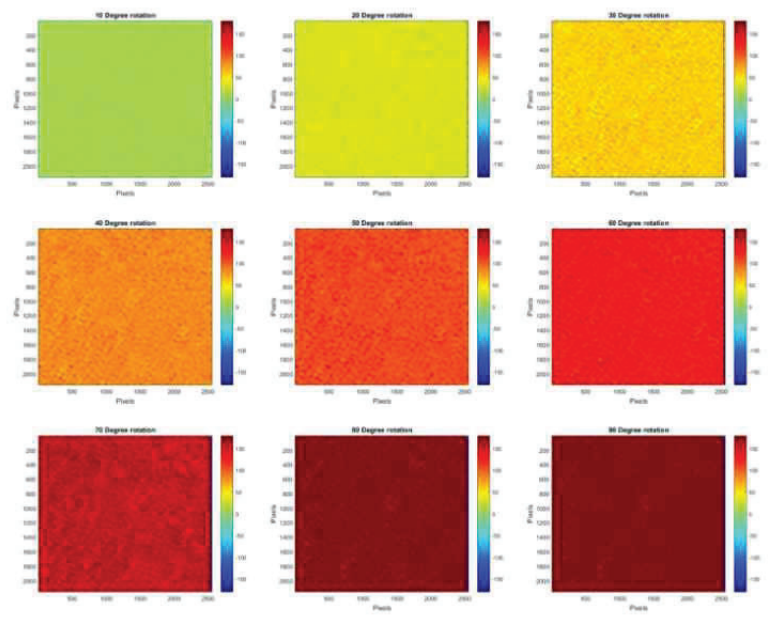

Figure 1. Spatial phase difference map measurement results when the optic axis angle of an achromatic QWP is varying from $-55^{\circ}$ with an interval of $10^{\circ}$.

To verify that the proposed snapshot imaging polarimetry can provide a reliable spatial phase 
difference, we compared the spatial $\Delta(\mathrm{x}, \mathrm{y})$ based on the proposed system and the commercial polarimeter based on pixel measurement at different position as depicted in Fig. 2. Figure 2 illustrates the spatial phase measurement based on pixel at $(x, y)=512,1024$ and 2048, respectively. They are in good agreement. The experimental results confirm that the proposed snapshot imaging polarimeter can provide an accurate $\Delta(\mathrm{x}, \mathrm{y})$.

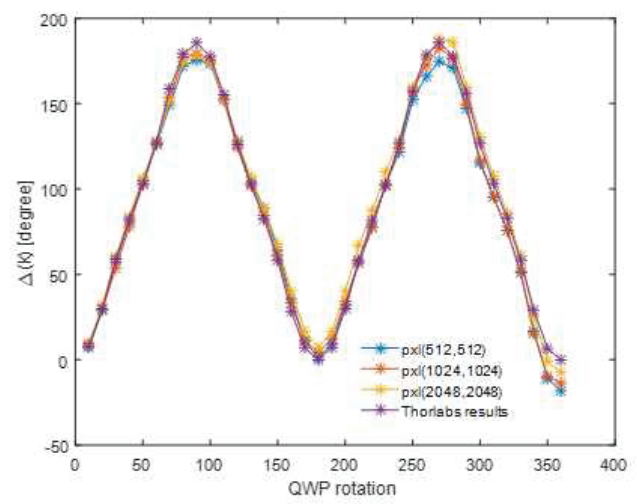

Figure 2. Measurement results comparison of $\Delta s$ between the proposed and a commercial polarimeter by varying the QWP rotation angle.

Funding. This work was supported by Samsung Research Funding \& Incubation Center of Samsung Electronics under Project Number SRFC-TA1703-11.

\section{References}

[1] J. S. Tyo, D. L. Goldstein, D. B. Chenault, and J. A. Shaw, "Review of passive imaging polarimetry for remote sensing applications," Appl. Opt. 45, 54535469 (2006).

[2] L. Asinovski, D. Beaglehole, and M. T. Clarkson, "Imaging ellipsometry: quantitative analysis," Phys. Stat. sto. (a), 1-8 (2008).

[3] J. F. de Boer, T. E. Milner, M. J. C. van Gemert, J. S. Nelson, "Two-dimensional birefringence imaging in biological tissue by polarization-sensitive optical coherence tomography," Opt. Lett. 22, 934-936 (1997).
[4] G. P. Nordin, J. T. Meier, P.C. Deguzman, and M. W. Jones, "Micropolarizer array for infrared imaging polarimetry," J. Opt. soc. Am. A 16, 1168-1174 (1999).

[5] T. Colomb, P. Dahlgren, D. Beghuin, E. Cuche, P. Marquet, and C. Depeursinge, "Polarization imaging by use of digital holography”, Appl. Opt. 41, 27-37 (2002).

[6] K. Oka and T. Kaneko, "Compact complete imaging polarimeter using birefringent wedge prisms," Opt. Exp. 11, 1510-1519 (2003).

[7] M. W. Kudenov, M. J. Escuti, E. L. Dereniak, and K. Oka "White-light channeled imaging polarimeter using broadband polarization gratings," Appl. Opt.50, 22832293 (2011) .

[8] D. G. Abdelsalam, R. Magnusson, and D. Kim, "Single-shot dual wavelength digital holography based on polarizing separation," Appl. Opt. 50, 3360-3368 (2011).

[9] M. Takeda, H. Ina, and S. Kobayashi, "Fouriertransform method of fringe-pattern analysis for computer-based topography and interferometry," J. Opt. Soc. Am. 72, 156 -160 (1982). 


\title{
Spectroscopic Ellipsometry Study on Aluminium-Doped Zinc Oxide Thin Films Prepared via DC Magnetron Sputtering and HiPIMS
}

\author{
Donyawan Chittinan', Tossaporn Lertvanithphol ${ }^{2}$, Kittikhun Seawsakul ${ }^{3}$, Prayoon Songsiriritthigul ${ }^{3}$, Phitsanu \\ Poolcharuansin $^{4}$, Mati Horprathum ${ }^{2}$, Prathan Buranasiri ${ }^{1 *}$ \\ ${ }^{1}$ Department of Physics, Faculty of Science, King Mongkut's Institute of Technology Ladkrabang, Bangkok, 10520, Thailand \\ ${ }^{2}$ National Electronics and Computer Technology Center, National Science and Technology Development Agency (NSTDA), Pathum Thani 12120, \\ Thailand \\ ${ }^{3}$ NANOTEC-SUT Center of Excellence on Advanced Functional Nanomaterials and School of Physics, Suranaree University of Technology, Nakhon \\ Ratchasima 30000, Thailand \\ ${ }^{4}$ The Technological Plasma Research Unit, Department of Physics, Mahasarakham University, Maha Sarakham, 44150, Thailand \\ Authore-mail address: prathan.bu@kmitl.ac.th
}

\begin{abstract}
Spectroscopic ellipsometry was used to investigate the properties of aluminium-doped zinc oxide (AZO) thin films deposited on silicon (100) substrate via magnetron sputtering system. The different techniques were used to fabricate including conventional DC magnetron sputtering and high-power impulse magnetron sputtering (HiPIMS). The ellipsometric spectra of the prepared samples were performed in the range of 1.75 to $3.5 \mathrm{eV}$ with $0.025 \mathrm{eV}$ intervals at incident angle of 70 degree. Two different optical models based on Cauchy and Tauc-Lorentz functions were used to fit and compared. The results showed that the Tauc-Lorentz based model was better to fit and extract the properties of interest including thin film thickness and refractive index (n). In addition, the fieldemission scanning electron microscopy (FE-SEM) images were used to confirm the results of thickness and ensure the using of Tauc-Lorentz model.
\end{abstract}

\section{Introduction}

Transparent conducting oxide (TCO) films is widely used in severally applications e.g. photovoltaics, flat panel displays, semiconductor lasers, light emitting diodes and solar cell [1]. In general, there are some materials that used as TCOs including indium-doped tin oxide (ITO), fluorine-doped tin oxide (FTO) and aluminum-doped zinc oxide (AZO). However, the AZO got more interested due to its high stability, low resistivity, high optical transmittance and low cost. The AZO thin film that could be fabricated by several techniques, such as evaporation, chemical vapor deposition, solgel method, pulsed laser deposition and sputtering [2]. Among these methods, direct current (DC) magnetron sputtering deposition was widely used with AZO films fabrication because of its repeatability, low temperature and large scale process. However, the good AZO films must had high homogeneity and high density. From this point, high-power impulse magnetron sputtering (HiPIMS) could be an appropriate technique to fabricate AZO. In addition, it has been shown that HiPIMS reduced the hysteresis effect which increased homogeneity and packing density of the film [3].

\section{Experimental Details}

AZO thin films were fabricated on silicon (100) substrates by homemade magnetron sputtering system. The 3 inches diameter of AZO material and $99.999 \%$ purity argon were used as sputtered target and sputtered gas, respectively. Prior to deposition, the system was pumped down to the base pressure of $5 \times 10^{-6}$ Torr by rotary and turbomolecular pumps. The AZO films were prepared with different technique including conventional DC magnetron sputtering, labelled sample as "AZO-DC", and high-power impulse magnetron sputtering (HiPIMS), labelled sample as "AZO-HiPIMS", respectively. During deposition for $20 \mathrm{~min}$, Ar flow rate and operating pressure were respectively set at $80 \mathrm{sccm}$ and $4.2 \times 10^{-3}$ mbar for AZO-DC while those of AZO-HiPIMS were respectively set at $100 \mathrm{sccm}$ and $2.1 \times 10^{-3}$ mbar. For AZO-DC sample, the DC bias power was constantly set at $100 \mathrm{~W}$. For AZO-HiPIMS, the bias power was set its frequency at $100 \mathrm{~Hz}$ while the duty cycle was set at $400 \mu \mathrm{s}$. The as-deposited films were characterized by spectroscopic ellipsometer (J.A. Woollam, Inc.; VASE). The ellipsometric spectra were performed in the range of 1.75-3.5 eV with $0.025 \mathrm{eV}$ intervals at 70 degree. The optical model of silicon substrate $/ 2 \mathrm{~nm}$ of Si native oxide/AZO thin film/surface roughness, which the optical properties of AZO layer was defined by different function of Cauchy and Tauc-Lorentz (TL) [4], was used to fit with the measured spectra and extract the properties. In addition, the thickness results were confirmed by field-emission scanning electron microscopy (FE-SEM) images in cross-sectional view.

\section{Results \& Discussions}

The measured ellipsometric spectra were fitted with both Cauchy and TL based optical models then compared together as shown in Fig. 1 and 2. The results showed that the TL based optical model represented the best fit with the measured $\Psi$ and $\Delta$ spectra. This indicated that the TL based model was a suitable model for AZO thin films. From the TL model, the thickness of AZO-DC and AZO-HiPIMS were determined as 337.4 and $313.8 \mathrm{~nm}$, respectively. Fig. 3(a) showed

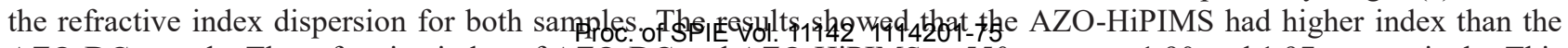


thickness, the prepared samples were characterized by FE-SEM. The film thicknesses obtained from both techniques were in good agreement as shown in Fig 3(b).
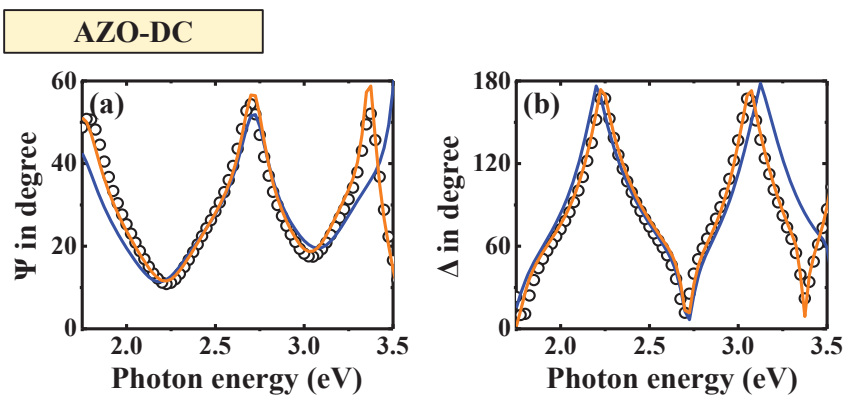

OMeasured - Cauchy - Tauc-Lorentz

Fig. 1. Measured and fitted $\Psi$ and $\Delta$ parameters from Cauchy and TL models for the AZO-DC sample
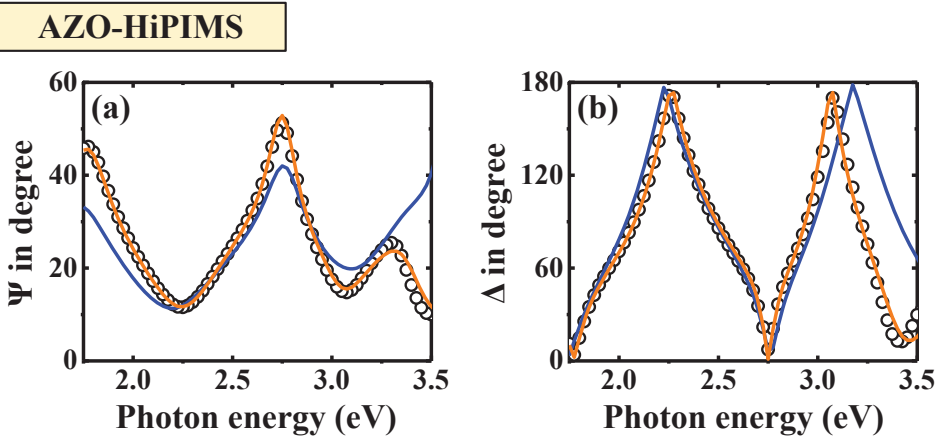

OMeasured - Cauchy - Tauc-Lorentz

Fig. 2. Measured and fitted $\Psi$ and $\Delta$ parameters from Cauchy and TL models for the AZO-HiPIMS sample
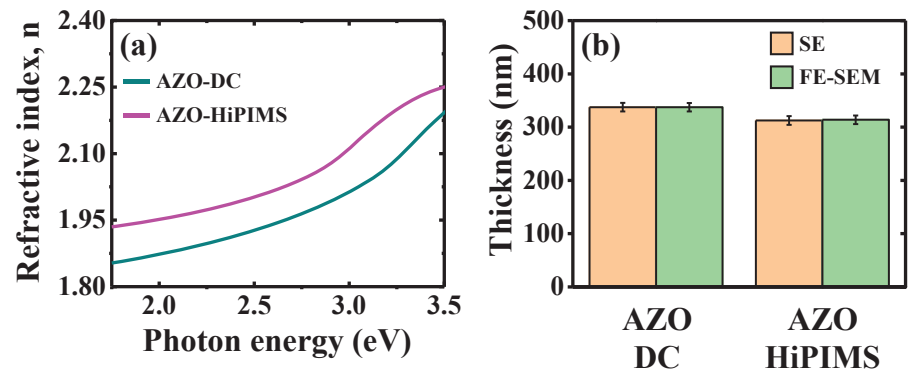

Fig. 3. Comparison of (a) refractive index spectra between AZO-DC and AZO-HiPIMS and (b) thickness results from SE and FE-SEM.

\section{Conclusion}

The AZO thin films were fabricated by conventional DC magnetron sputtering and HiPIMS techniques. The prepared films were characterized for their properties by SE. The optical models based on Cauchy and TL were used and compared. The results showed that the TL model was an appropriate model for AZO films. The thicknesses and refractive index spectra were extracted from the model. The HiPIMS film had higher index than the film from conventional DC. This indicated that the AZO film could be increased their density by HiPIMS technique. In addition, the thickness results from SE were compared with those from FE-SEM to ensure the extract properties.

\section{References}

[1] M. Mickan et al., "Room temperature deposition of homogeneous, highly transparent and conductive Al-doped ZnO films by reactive high power impulse magnetron sputtering", Sol. Energy Mater. Sol. Cells 157, 742-749 (2016)

[2] G. Xiao-Yong, C. Chao, Z. Sa, "Optical properties of aluminum-doped zinc oxide films deposited by direct-current pulse magnetron reactive sputtering”, Chin. Phys. B 23, 1-5 (2014).

[3] V. Sittinger et al., "Applications of HIPIMS metal oxides" Thin Solid Films 548, 18-26 (2013)

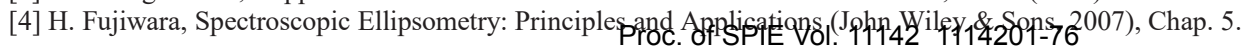




\title{
Imaging elliprometry of porous silicon
}

\author{
Lianhua Jin*a $^{* a}$ Taiki Akiyama ${ }^{\mathrm{a}}$, Yuki Iizuka ${ }^{\mathrm{a}}$, Eiichi Kondoh ${ }^{\mathrm{a}}$, Benard Gelloz \\ ${ }^{a}$ Faculty of Engineering, University of Yamanashi, Yamanashi 400-8511, Japan \\ ${ }^{\mathrm{b}}$ Faculty of Engineering, Nagoya University, 464-8601 Nagoya, Japan
}

\begin{abstract}
Imaging ellipsometry studies of $\mathrm{n}+$-type porous silicon layers are presented. The morphology of $\mathrm{n}+$-type porous silicon is discussed in accordance with two-dimensional measurement results of refractive index and thickness. The results show that imaging ellipsometry is a powerful technique for materials characterization.
\end{abstract}

Keywords: Imaging ellipsometry, Porous silicon, Material, Refractive index

\section{Introduction}

The optoelectronic functions of porous silicon (PS), particularly its photoluminescence and electroluminescence are attractive and promising features $[1,2]$. The optical properties of PS layers are determined by their nanostructures. PS nanostructures are formed by electrochemical etching of silicon substrates in hydrofluoric acid (HF). Depending on doping level and type, the formation method of $\mathrm{PSi}$ and resultant nanostructures are different. For n- or n+-type silicon, the PS layer is obtained by anodization of P-doped silicon chip in HF with etching-side or backside illumination. The formed $n+$-type PS nanostructure is sometimes not uniform. To investigate the morphology of n+-type PS, imaging ellipsometry studies has been carried on. The measurement results show that imaging ellipsometry is a powerful tool to characterize materials.

\section{Measurements}

\section{Experimental setup}

Figure 1 shows the configuration of the imaging ellipsometry system [3-4] built by our group. The light from xenon lamp passes thorough an optical filter (for $530,590,650,720,780 \mathrm{~nm}$, respectively), a convergence lens system, a linear Gran-Thompson polarizer, and then a compensator. The reflected light from the sample surface

*lianhua@yamanashi.ac.jp; phone 81 55-220-8448 passes through an analyzer and an Offner imaging system (NA=0.07), and then is collected by a CMOS device $(2592 \times 1944$ pixels, 12 Bit). The detection area of the CMOS device is $6.4 \times 4.8 \mathrm{~mm}^{2}$. The incident angle is $50^{\circ}$. The lateral resolution of the imaging system is about $5 \mu \mathrm{m}$ at the wavelength of $530 \mathrm{~nm}$. The image plane of the CMOS device was carefully adjusted to receive the entire image in focus. Rotating compensator method was employed to obtain the ellipsometric parameters DEL $(\Delta)$ and PSI $(\psi)$ of samples. These parameters are defined by,

$$
\tan (\Psi) \exp (j \Delta)=\frac{r_{p}}{r_{s}},
$$

where $r_{p}$ and $r_{s}$ are the Fresnel reflection coefficients of $p$ and $s$ polarized light, respectively. By analyzing the relationships of DEL and PSI with refractive index and film thickness of samples, we can investigate the sample morphology.

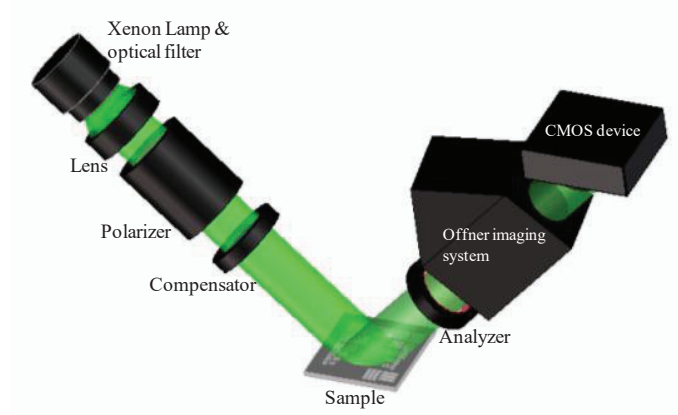

Figure 1. Configuration of imaging ellipsometry setup 


\section{2. n+-type porous silicon preparation}

The PSi layer was formed from P-doped, (111)oriented, and $<0.018 \Omega \mathrm{cm}$ silicon substrates with an $\mathrm{Al}$ back contact. Anodization was performed in a mixture solution of aqueous HF (46 wt.\%) and ethanol (approx. $2: 5$ ) at a constant current density of $3 \mathrm{~mA} / \mathrm{cm}^{2}$. The layer with thickness of approximately $0.5 \mu \mathrm{m}$ was obtained by etching $200 \mathrm{~s}$ under illumination (by a cold light source) of 2000 LUX (see Fig. 2a). Figure 2b and 2c show the optical image of the formed PSi layer and its crosssectional electron micrograph image, respectively. The optical image of Fig. $2 \mathrm{~b}$ indicates that the nanostructures of n+-type PS layer is not uniform.

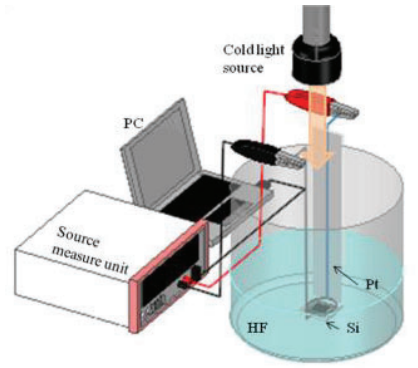

(a)

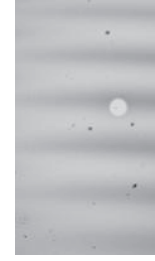

(b)

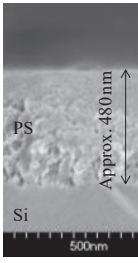

(c)
Figure 2. (a) Anodization setup for sample preparation.

(b) Optical image of n+-type PS layer. (c) Crosssectional electron micrograph image of the sample.

\section{Ellipsometric measurement results and}

\section{discussion}

Imaging ellipsometric measurement was carried out at above-mentioned five wavelengths. Figures $3 \mathrm{a}$ and $3 \mathrm{~b}$ display DEL and PSI maps of the region in Fig. $2 b$ at the wavelength of $650 \mathrm{~nm}$. To obtain two-dimensional refractive index and thickness of n+-type PS, DEL and PSI maps of five wavelengths were analyzed. Figures $4 \mathrm{a}$, $4 \mathrm{~b}$ and $4 \mathrm{c}$ illustrate real refractive index, extinction coefficient (at $\lambda=650 \mathrm{~nm}$ ), and thickness maps of the sample, respectively. The average thickness is about 470 $\mathrm{nm}$, which is very close to the value predicted from Fig. 2c. The thickness changes irregularly over the measurement region. The real refractive indices and extinction coefficients changes periodically, which is corresponding to the intensity distribution shown in Fig. $2 \mathrm{~b}$. It indicates that the thickness of the PS layer is mainly decided by the etching time. The nanostructure of the PSi layer is, however, strongly affected by the illumination. During formation of PS, multi-reflection interference occurs by the thin layer, which induces periodic distribution of intensity across the sample. This may be one of the reasons which cause periodic nanostructures formation.
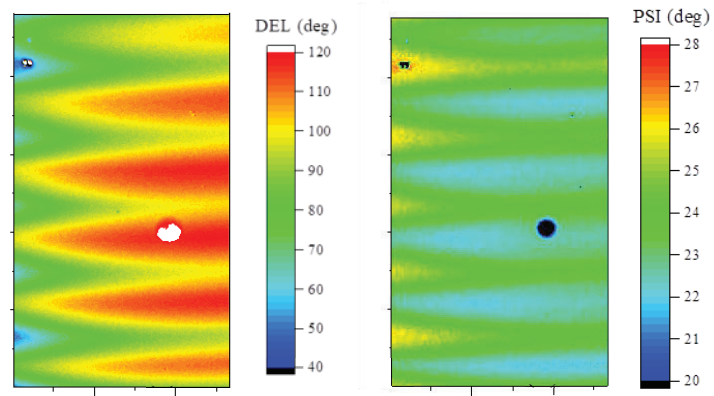

Figure 3. (a) Ellipsometric DEL map and (b) the corresponding PSI map of n+-type PS layer at the wavelength of $650 \mathrm{~nm}$.

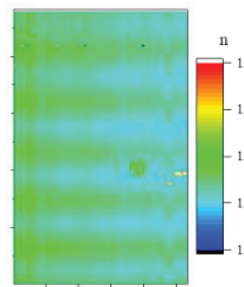

(a)

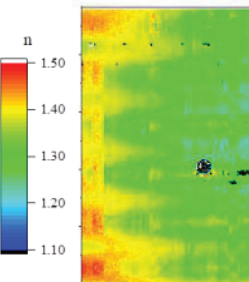

(b)

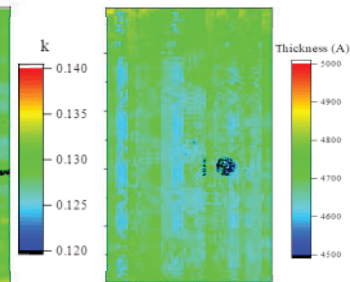

(c)
Figure 3. (a) Real refractive index map, (b) the extinction coefficient map at the wavelength of 650 $\mathrm{nm}$, and (c) thickness map.

\section{Conclusions}

Imaging ellipsometry was used to characterized $n+-$ type PS layer. The single point measurement ellipsometry is not available for investigation of the optical properties of non-uniform layers or micro-patterned samples. The measurements results provide imaging ellipsometry as a powerful tool for characterization of new materials.

\section{References}

[1] Cullis, A. G., Canham, L. T., Calcott, P. D. J., “The 
structural and luminescence properties of porous silicon,” J. Appl. Phys. 82, 909-965 (1997).

[2] Gelloz, B. and Koshida, N., "Electroluminescence with high and stable quantum efficiency and low threshold voltage from anodically oxidized thin porous silicon diode" J. Appl. Phys. 88, 4319-4324 (2000).

[3] Jin, L., Tanaka, T., Kondoh, E., Gelloz, B., Sano, K., Fujio, I., Kajiyama, Y., Uehara, M., "Rotatable Offner imaging system for ellipsometric measurement," Rev. Sci. Instrum. 88, 013704 (2017).

[4] Jin, L., Tanaka, T., Kondoh, E., Gelloz, B., Uehara, M., "Extracting calibrated parameters from imaging ellipsometric measurement," Jpn. J. Appl. Phys. 56, 116602 (2017). 


\title{
Effect of oxide layer thickness on polarization mitigation*
}

\author{
Suchandra Banerjee*a, Russell Chipman ${ }^{\mathrm{b}}$, Nathan Hagen ${ }^{\mathrm{c}}$, Yukitoshi Otani ${ }^{\mathrm{a}, \mathrm{c}, \mathrm{d}}$ \\ ${ }^{a}$ Graduate School of Engineering, Utsunomiya University, Utsunomiya, Tochigi, 321-8585, Japan, \\ ${ }^{\mathrm{b}}$ College of Optical Sciences, University of Arizona, Tucson, AZ 85721, USA, \\ ${ }^{\mathrm{c}}$ Department of Optical Engineering, Utsunomiya University, Utsunomiya, Tochigi, 321-8585, Japan, \\ ${ }^{\mathrm{d}}$ Center for Optical Research and Education, Utsunomiya University, Utsunomiya, Tochigi, \\ 321-8585, Japan
}

\begin{abstract}
We analyze the influence of oxide layer thickness on polarization cancellation using crossed fold mirrors. We measure the linear retardance of three individual aluminum coated mirrors over the visible to near infra-red region by employing a spectroscopic Mueller matrix polarimeter. By fitting the measured linear retardance data with the theoretical model described by Fresnel's law, the native oxide layer thickness of three mirrors are determined as $4.40 \mathrm{~nm}, 5.75 \mathrm{~nm}$, and 4.10 $\mathrm{nm}$ respectively. We perform polarization cancellation by using crossed fold mirrors. The oxide layer thickness difference of $1.35 \mathrm{~nm}$ between the first two mirrors limits the polarization cancellation to $0.65^{\circ}$ of retardance at $650 \mathrm{~nm}$. We show that the first and last fold mirrors having nearly same amount of oxide layer thickness reduces the residual quantity to $0.15^{\circ}$ at $650 \mathrm{~nm}$.
\end{abstract}

Keywords: Polarization, Mueller matrix polarimeter, oxide layer, crossed fold mirrors, linear retardance.

\section{Introduction}

Reflecting surfaces are widely used in optical systems to make the whole system compact. Instruments from space telescopes to ground telescopes, remote sensing spectrometers, microlithography all use multiple fold mirrors ${ }^{[1]}$. Traditional materials for metal mirrors include aluminum, gold and silver for optical applications. Among them, aluminum (Al) coatings are common due to high reflectivity over a wavelength range. For an ideal mirror, the reflection coefficients of $s$ and $p$ polarizations are equal, where a metal coated mirror reflects more $s$ polarized light than $p$ polarized light at a non-normal angle of incidence. The difference of complex amplitude reflection coefficients creates a phase difference between $s$ and $p$ polarized lights. This unwanted linear retardance can deteriorate image quality ${ }^{[1]}$.

Lam et al. ${ }^{[2]}$ reported that polarization aberration can be balanced by using crossed fold mirrors. Gold mirrors are selected in their simulation process which in practical makes the system expensive and can be used only in infra-red region.

Bare $\mathrm{Al}$ mirrors react with oxygen in air, creating a *suchandra@opt.utsunomiya-u.ac.jp; thin oxide layer on the top of the mirror surface. This can further increase the proper linear retardance which is excluded from geometrical transformation.

In this manuscript, we determine the naturally formed oxide layer thickness by fitting the linear retardance of aluminum mirrors with a theoretical model for the Fresnel's reflection coefficients. We report the effect of oxide layer thickness on the cancellation of linear retardance using two different pairs of crossed fold mirrors.

\section{Theoretical background}

We employ Polarization ray tracing $(P)$ matrices ${ }^{[3]}$ to determine the polarization cancellation and analyze the polarizing state evolution for each mirror surface. $P$ matrix is generalized $3 \times 3$ Jones $(J)$ matrix which is obtained by using,

$$
P_{q}=O_{q, \text { out }} J O_{q, \text { in }}^{-1},
$$

here $q$ represents the surface number and $O_{q}$,out and $O_{q, \text { in }}$ are the exiting and incident orthogonal matrices respectively which transform the Jones matrix of mirror from local to global coordinate system. Orthogonal matrix is functions of orthogonal polarizations $(s, p)$ and propagation vector $(k)$, 


$$
O_{q, \text { in }}=\left(\begin{array}{lll}
s_{q, x} & p_{q-1, x} & k_{q-1, x} \\
s_{q, y} & p_{q-1, y} & k_{q-1, y} \\
s_{q, z} & p_{q-1, z} & k_{q-1, z}
\end{array}\right), O_{q, \text { out }}=\left(\begin{array}{ccc}
s_{q, x} & p_{q, x} & k_{q, x} \\
s_{q, y} & p_{q, y} & k_{q, y} \\
s_{q, z} & p_{q, z} & k_{q, z}
\end{array}\right),
$$

The propagation vectors $(k)$ in xyz coordinate system are,

$$
k_{1}=\{0,0,1\}, k_{2}=\{0,1,0\} \text {, and } k_{3}=\{1,0,0\} .
$$

Orthogonal polarization components $s$ and $p$ depend on the propagation vector as,

$$
\begin{gathered}
s_{q}=\left(k_{q-1} \times k_{q}\right) /\left|k_{q-1} \times k_{q}\right|, \\
p_{q, \text { in }}=\left(k_{q-1} \times s_{q}\right) /\left|k_{q-1} \times s_{q}\right|, \text { and } \\
p_{q, \text { out }}=\left(k_{q} \times s_{q}\right) /\left|k_{q} \times s_{q}\right| .
\end{gathered}
$$

Jones matrix in Eq. 1 is the mirror's polarization property and can be written as,

$$
J=\left(\begin{array}{ccc}
r_{s} & 0 & 0 \\
0 & -r_{p} & 0 \\
0 & 0 & 1
\end{array}\right)
$$

where, $r_{s}$ and $r_{p}$ are the apparent reflection coefficients derived from the Fresnel's model shown in Fig.1. It can be formed as,

$$
r_{s}=\frac{r_{01 s}+r_{12 s} e^{\mathrm{i} \phi}}{1+r_{01 s} r_{12 s} e^{\mathrm{i} \phi}} \text {, and } r_{p}=\frac{r_{01 p}+r_{12 p} e^{\mathrm{i} \phi}}{1+r_{01 p} r_{12 p} e^{\mathrm{i} \phi}},
$$

where $r_{01}$ and $r_{12}$ are the Fresnel reflection coefficients for medium 0 to 1 and for medium 1 to 2 respectively for $s$ and $p$ polarized light. $\phi$ is the phase change of light which occurs due to oxide layer thickness in this model and it is functions of refractive index $\left(n_{1}\right)$, thickness $(d)$ of oxide layer and can be represented as,

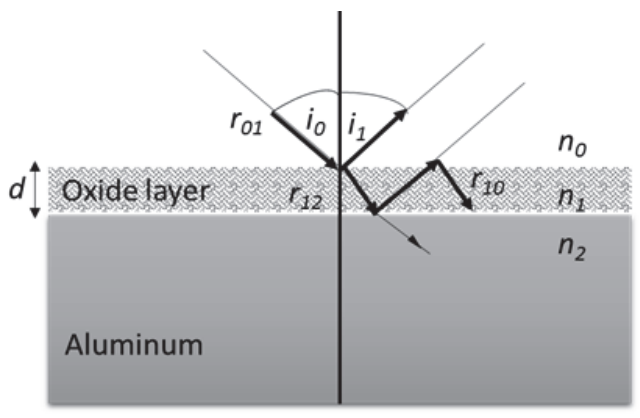

Figure 1. Theoretical model of Fresnel's law by considering oxide layer thickness on the top of $\mathrm{Al}$ mirror.

$$
\phi=\frac{2 \pi}{\lambda} n_{1} d \cos i_{0} .
$$

By using Eq. (1-7), overall $P$ matrix is determined by cascading $1^{\text {st }}$, and $2^{\text {nd }}$ mirrors $P$ matrices, shown in Eq. 8 . By using singular value decomposition, $P$ matrix is decomposed into retardance $\left(M_{R}\right)$ and diattenuation matrices $\left(M_{D}\right)$ as,

$$
P=P_{2} P_{1}=\mathrm{M}_{D} \mathrm{M}_{\mathrm{R}} .
$$

Retarder matrix is an Unitary matrix whose Eigen values $\left(\lambda_{1}, \lambda_{2}, \lambda_{3}\right)$ are complexed valued. Two of the three Eigen values denote fast and slow axes whose argument difference is the linear retardance $(\delta)$ after cancellation which is,

$$
\delta=\arg \left[\lambda_{1}\right]-\arg \left[\lambda_{2}\right]
$$

\section{Experimental set up and results}

An Axometrics Mueller matrix polarimeter is used for the measurement of polarization properties of Al mirror. Fig. 2 shows the schematic diagram of experimental set up. It consists of polarization state generator (PSG) and polarization state analyzer (PSA). The PSG comprises a polarizer $(\mathrm{P})$ oriented at $0^{\circ}$ and a rotating retarder $\left(\mathrm{R}_{1}\right)$ having rotational angle $\theta$. Another rotating retarder $\left(\mathrm{R}_{2}\right)$ having rotational angle $5 \theta$ and an analyzer (A) oriented at $0^{\circ}$ are placed in PSA. The system uses a Xenon arc lamp white light source and retrieves the polarization parameters as a function of wavelength. The Mueller matrix polarimeter is set at reflection mode and three aluminum coated mirrors $\left(\mathrm{M}_{1}, \mathrm{M}_{2}\right.$, and $\left.\mathrm{M}_{3}\right)$ are placed individually at $45^{\circ}$ angle of incidence.

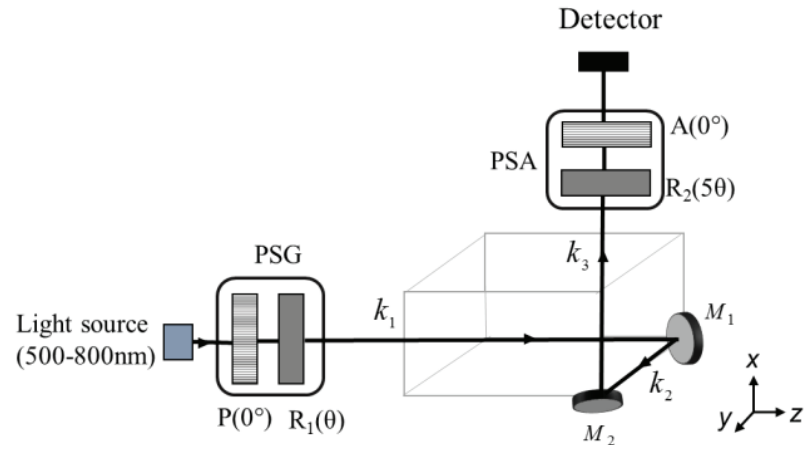

Figure 2. Experimental setup for measurement of polarization cancellation using two crossed fold mirrors 


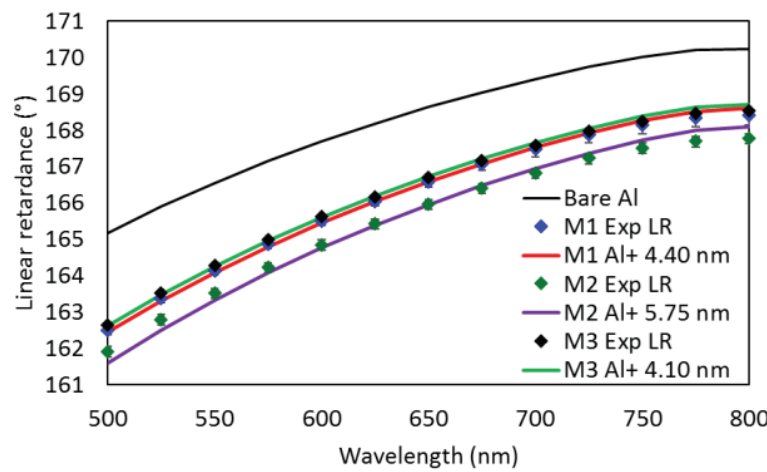

Figure 3. Linear retardance of bare aluminum mirror (obtained from theory) and real aluminum mirrors with measured (dots) and fitted (curves) oxide layer thickness.

Measured linear retardance of three individual mirrors are shown in Fig. 3 over spectral range $500 \mathrm{~nm}$ to $800 \mathrm{~nm}$. The theoretical value of linear retardance for a bare aluminum mirror is shown by black curve. The measured linear retardance of the mirrors are also shown in Fig. 3 and a mismatch is clearly visible between the theoretical and measured linear retardance values due to natural oxide layer that has formed on top of the aluminum mirrors. By using Eq. [6-7], the measured linear retardance data of the real aluminum mirrors are fitted by considering oxide layer thickness. The resulting oxide layers for three individual mirrors are estimated as $4.40 \mathrm{~nm}, 5.75 \mathrm{~nm}$ and $4.10 \mathrm{~nm}$ respectively.

For cancellation of linear retardance, two individual sets of crossed fold mirrors are chosen. The first set of crossed fold aluminum mirrors $\mathrm{M}_{1}$ and $\mathrm{M}_{2}$ have different thicknesses of naturally formed oxide layers $4.40 \mathrm{~nm}$ and5.75 $\mathrm{nm}$. The mirror pair is placed in the sample path of the Axometrics polarimeter. The $1.35 \mathrm{~nm}$ difference of natural oxide layer thickness yields a residual amount of

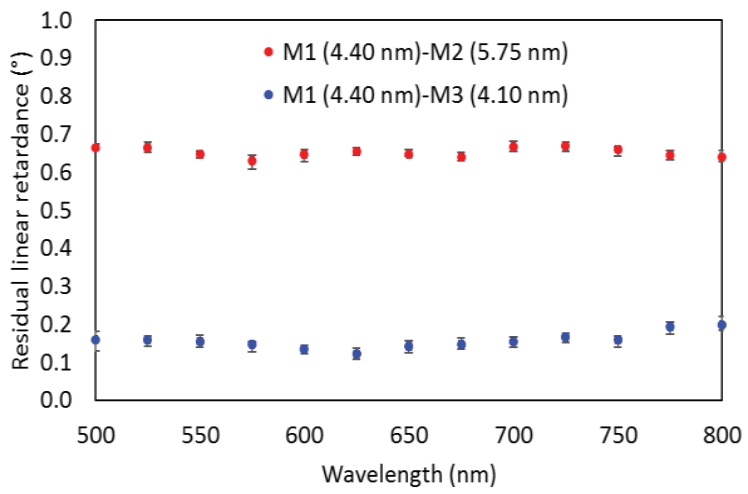

Figure 4. Impact of oxide layer thickness on linear retardance cancellation using crossed fold aluminum mirrors. linear retardance of $0.65^{\circ}$ at $650 \mathrm{~nm}$ after cancellation as shown in Fig. 4.

The second set of crossed fold $\mathrm{Al}$ mirrors $\mathrm{M}_{1}$ and $\mathrm{M}_{3}$ have oxide layer thicknesses that are close to one another i.e. $4.40 \mathrm{~nm}$ and $4.10 \mathrm{~nm}$ respectively, so that the residual amount of linear retardance after cancellation goes down to $0.14^{\circ}$ at $650 \mathrm{~nm}$ as shown in Fig. 4. Since Aluminum mirrors are dispersive in nature, linear retardance of individual mirror is wavelength dependent as shown in Fig. 3. The difference in linear retardance between two mirrors are equal over spectrum as evident from Fig. 3. In support of this, Fig. 4 demonstrates that after cancellation of linear retardance by using two fold mirrors, the residual amount of linear retardance is almost constant over 500$800 \mathrm{~nm}$ for both cases.

\section{Discussion and conclusion}

The native aluminum oxide layer thickness of $\mathrm{Al}$ mirrors is determined by measuring their polarizing property of aluminum mirrors. The amount of oxide layer on aluminum mirror may vary from mirror to mirror and this has been confirmed by measuring three individual aluminum mirrors' linear retardance. The impact of the naturally formed oxide layer on polarization cancellation using crossed fold mirrors is demonstrated. For accurate polarization measurements, polarization cancellation needs to be performed in the optical systems where fold mirrors are highly used and polarimetry is combined with the systems like telescope, spectrometer, and microlithography.

\section{References}

1) James B. Breckinridge, Wai Sze T. Lam and Russell A. Chipman, "Polarization Aberrations in Astronomical Telescopes: The Point Spread Function," PSAP,127, 445-468 (2015).

2) Wai Sze, Tiffany Lam, and Russell Chipman, "Balancing polarization aberrations in crossed fold mirrors," Appl. Opt. 54, 3236-3245 (2015).

3) Garam Yun, Stephen C. McClain, and Russell A. Chipman, "Three-dimensional polarization raytracing calculus II: retardance," Appl. Opt. 50, 2866-2874 (2011). 
High-Precise Optical Shape Measurement with Full-Field Heterodyne Interferometry Zhou Wu ${ }^{1,2,3^{*}}$, Wenxi Zhang ${ }^{1,2,3}$, Bin Xiangli ${ }^{1}$, Yang $\mathrm{Li}^{1,2,3}$, Xinxin Kong ${ }^{1,2}$

(1. Key Laboratory of Computational Optical Imaging Technology, CAS, NO.9

DengZhuang South Road, HaiDian District, Beijing, 100094, China

2.Academy of Opto-Electronics, Chinese Academy of Sciences, NO.9 DengZhuang

South Road, HaiDian District, Beijing, 100094, China

3. University of Chinese Academy of Sciences, NO.19, YuQuan Road, ShiJingShan

District, Beijing, 100049, China )

\begin{abstract}
This paper proposed a new high precise optical shape measurement system with full-field heterodyne interferometry. Optical elements with large aperture and long focal length have been widely used in high-end optical system, such as high-resolution telescope, deep ultra-violet lithography and so on. The shape accuracy of optical elements has a significant impact on the performance of these systems. When the surface shape of optical element with long focal length is measured by a conventional interferometer, environmental factors, such as vibration and airflow, often have a great influence on the measurement accuracy due to the long interference cavity.
\end{abstract}

To solve the problem, we proposed a small, high-stability, high-precision dynamic interferometer for surface shape measurement of optical element, using heterodyne technology to suppress the effects of vibration and airflow, self-developed high-performance AOFS (Acousto-optic frequency shifter) with Hertz-class to generate two coherent beams with a fixed beat frequency. One beam, as measuring light, is reflected by the surface under test and interfered with the reference beam. As a result, the shape information of the optical element surface is modulated in the interference signal. The array detector is used to detect the modulated signal, and the shape information could be obtained by digital demodulation. We also use fiber point diffraction technology to generate an ideal reference wavefront, and it can improve the absolute measurement accuracy of the system and miniaturize the dynamic interferometer. The schematic of full-field heterodyne interferometer is shown in Figure 1.

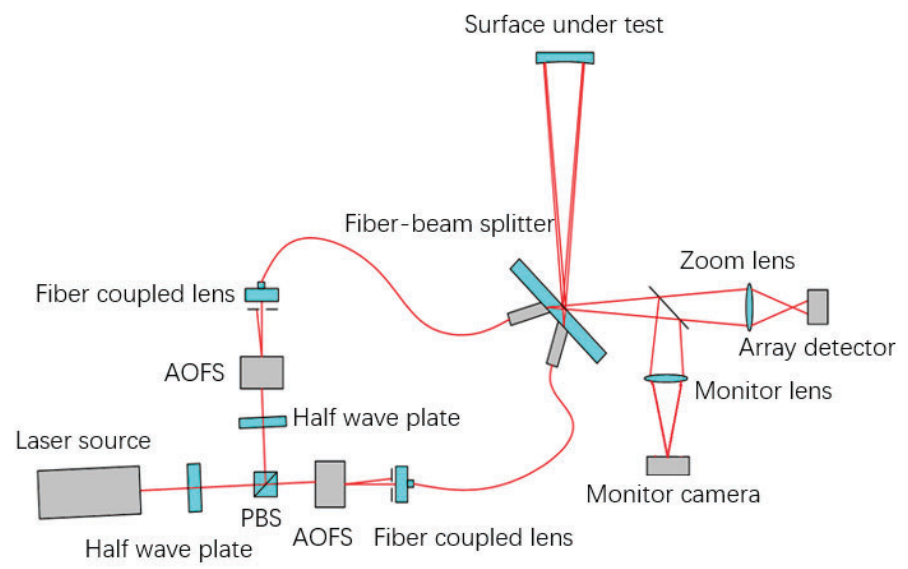

Figure 1. the schematic of full-field heterodyne interferometer
Proc. of SPIE Vol. 11142 1114201-83 
We adopt the scheme of Hertz-class continuous heterodyne phase shifting. Compared with traditional mechanical phase shifting technology, it has the advantages of accurate phase shift, good repeatability, high stability and low process difficulty. And high accuracy is easier to achieve, especially suitable for dynamic measurement of optical lens or system with long focal length and large aperture.

In dynamic interferometry, environmental vibration, airflow and detection noise usually conform to certain distribution laws. As the sampling time of the detection signal increases, the influence of the noise tends to mean value. Compared to traditional mechanical four-step phase shift or multi-step phase shift, heterodyne phase shift demodulates phase with higher precision. Through fast multicycle sampling combined with higher frequency domain analysis algorithm, vibration, laser energy fluctuation, detector shot noise, coherent noise and electronic noise can be effectively suppressed and it could further improve the measurement accuracy of the interferometer.

In order to verify the feasibility of the system for high-precision measurement of optical element with long focal length, we set up an experimental device to verify the repeat measurement accuracy of the interferometer. The experimental device is shown in Figure 2.

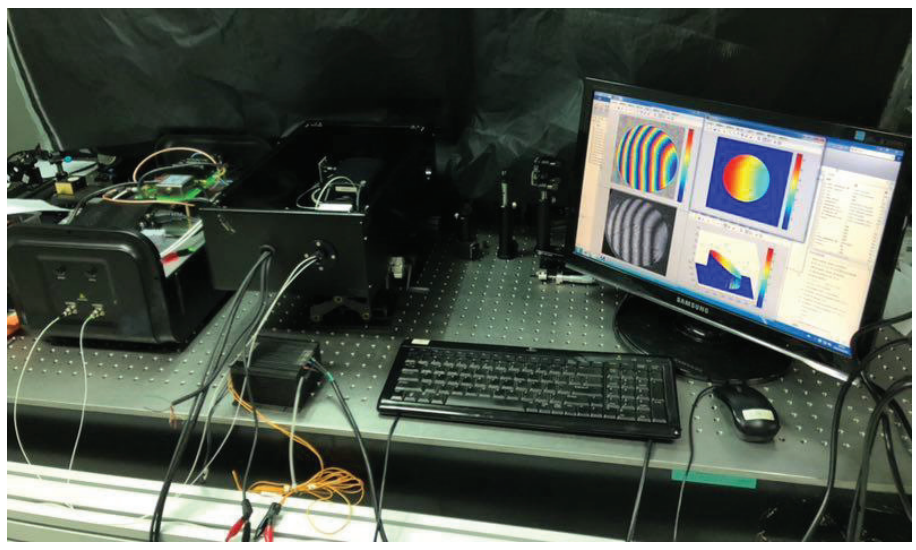

Figure 2. experimental device of full-field heterodyne interferometer

On this basis, we carried out a dynamic test of the shape of a mirror 10 meters away for measurement of 16 times. The test results are shown in Figure 3.

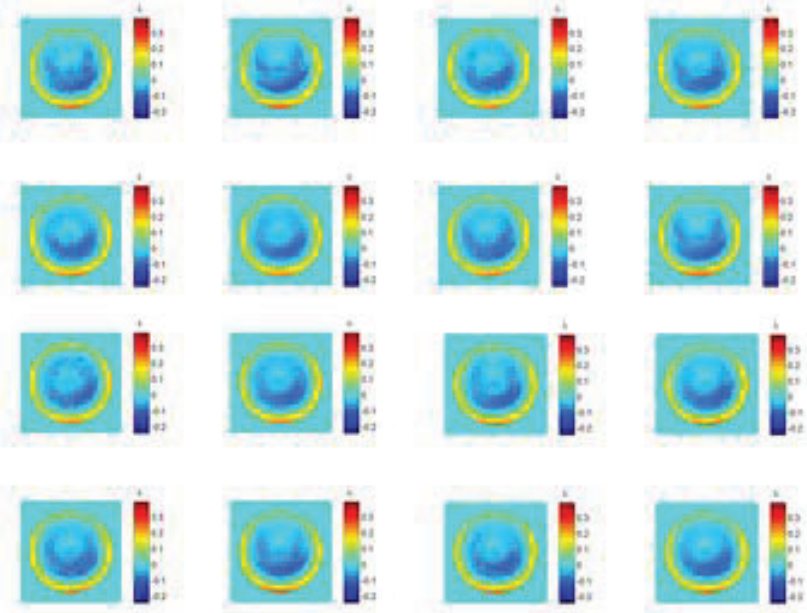

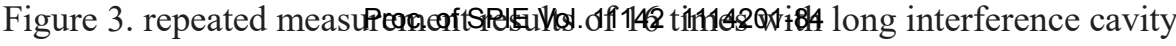


According to the experimental results, full-field heterodyne dynamic interferometer can measure the optical element with long focal length with high precision better than $\lambda / 1000$, and verify the ability of the interferometer to suppress vibration and air turbulence. It can solve the problem of shape measurement of optical element with long focal length and realize development of miniaturized interferometer. 


\title{
Optical profilometry of cylindrical openings for translucent objects
}

\author{
Lianhua Jin*a $^{\text {a }}$, Benard Gelloz ${ }^{\mathrm{b}}$, Toru Yoshizawa ${ }^{\mathrm{c}}$ \\ ${ }^{a}$ Faculty of Engineering, University of Yamanashi, Yamanashi 400-8511, Japan \\ ${ }^{b}$ Faculty of Engineering, Nagoya University, 464-8601 Nagoya, Japan \\ ${ }^{\mathrm{c}}$ NPO 3D Associates, Yokohama 230-0078, Japan
}

\begin{abstract}
A measurement method for the cylindrical openings profile of translucent objects is introduced. The optical probe consists of a semiconductor laser, a right circular conical mirror, and a camera embedded with a wide-field lens and a polarizer. The inner profile of an object made of resin was measured. The solution of problems occurring during measurement was discussed and practically verified.
\end{abstract}

Keywords: Optical profilometry, Translucent object, Cylindrical openings, Disk beam, Imaging process

\section{Introduction}

Owing to low cost and relatively easy process, resin materials become popular in manufacturing like small containers of daily life, and large pipes for water supply. Even through depending on the materials, the resin often scatters the light which passes through it, and renders the objects translucent. The dimension of resin-based objects with cylindrical or cylinder-like openings can be measured with instruments like vernier calipers. During measurement, the tips of gauges are adjusted to fit the inward-facing points of objects.

Here, we introduce an optical noncontact profilometry for cylindrical openings of resin-based objects by using disk beam probe. Similar probe was developed by several groups [1-3] for inner profile measurement of opaque objects. The inner surface of these objects was rough so that the image sensor could collect strong light scattered from the surface. The inner surface of resin-based objects is, however, smooth, which increase stray light, especially when the alignment is not perfect. In this work, the stray light was minimized efficiently by using polarizer embedded in the camera.

\section{Measurement}

\section{Measurement principle}

Figure 1 shows the optical arrangement of the disk beam probe. The collimated straight beam from a

*lianhua@yamanashi.ac.jp; phone 81 55-220-8448 laser source illuminates the right circular conical mirror. The laser beam reflected on the tip surface of the cone mirror forms a disk-shaped light sheet. When the disk beam sections the interior of the object, the intersection results in a closed line. A camera embedded with a widefield lens and a polarizer collects the image of profiles. The disk beam plane and sensor plane are parallel. In this design, the dimension $d_{o}$ of the object is obtained through following relationship with that $d_{i}$ of the image,

$$
d_{o}=d_{i} \frac{s_{o}}{s_{i}},
$$

where $s_{o}$ and $s_{i}$ are the distances from the lens to the disk beam plane and from the lens to the sensor plane, respectively.

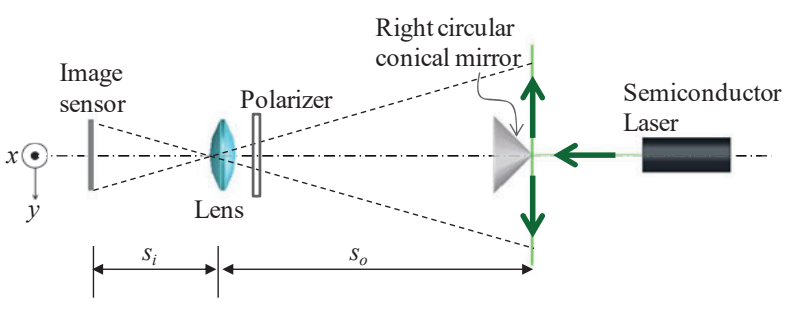

Figure 1. Configuration of optical arrangement of the disk beam probe.

\section{Translucent objects}

Different from the opaque objects, the translucent objects scatters light not only on the surface, also inside 
the material. The light collected by the camera includes both that scattered on the surface and that scattered inside the object. When the object surface is smooth, and alignment of the probe and the object is not perfect, strong specular reflection occurs on the surface out of focus plane and consequently stray light occurs around the intersection line. Figure $2 \mathrm{a}$ shows scattering phenomenon, which was taken from outside the opening of the translucent objects. Figure $2 b$ is the image collected through the probe. From Fig. 2b, we can see very clearly the stray light.

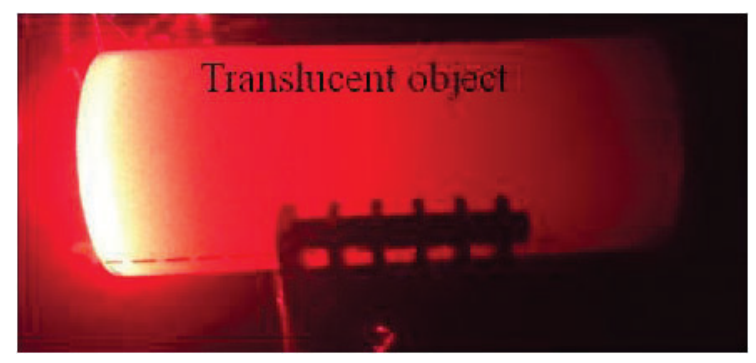

(a)

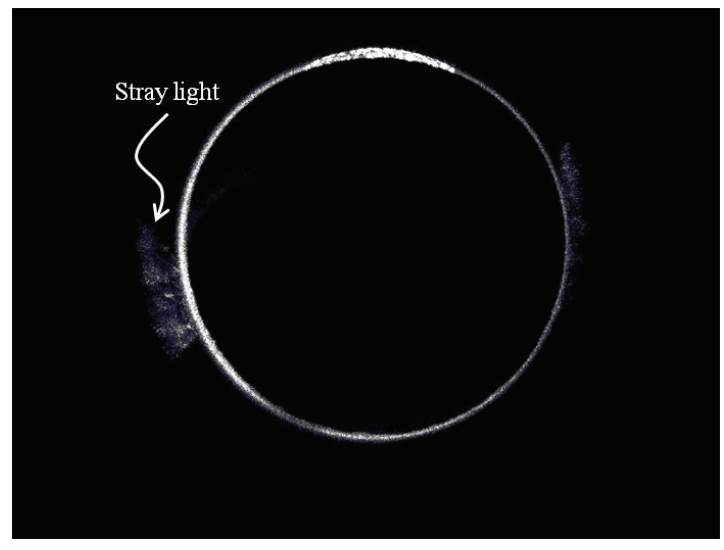

(b)

Figure 2. (a) Transmitted and (b) reflected lights by a translucent object were observed, while the disk beam illuminates it.

\section{Experiment results}

When light is reflected by the surface, it will be partially polarized [4]. The light reflected by the surface out of the focus plane induces stray light for the measurement probe. The stray light shown in Fig. $2 \mathrm{~b}$ is due to this reflection, and may be minimized by using a polarizer.

To measure the inner profile of this translucent object, we built the optical probe based on our previous work [2]. The diameter of base of the right circular cone mirror was $5.0 \mathrm{~mm}$. A semiconductor laser with wavelength of 635 $\mathrm{nm}$ (beam size $\approx 3 \mathrm{~mm}$ ) was employed for a light source. A CCD camera (PixeLink, 1024×768 pixels, 8 Bit, $4.65 \mu \mathrm{m}$ pixel pitch,) was used to capture the intersection profiles. The inverse longitudinal magnification $s_{o} / s_{i}$ of the lens is 13.00. A polarizer set in front of the lens is carefully adjusted until the stray light is minimized. Figure 3 shows the image collected after polarizer adjustment. From Fig. 3 , we can see the stray light decreased significantly. A threshold filter is applied to Fig. 3, and the profile coordinates are obtained on the image plane (see green round in Fig. 4). Through the regression analysis of the cloud of measurement points [3], the diameter of the opening was obtained as $33.48 \mathrm{~mm}$, while the results measured by the mechanical caliper was $33.55 \pm 0.09 \mathrm{~mm}$.

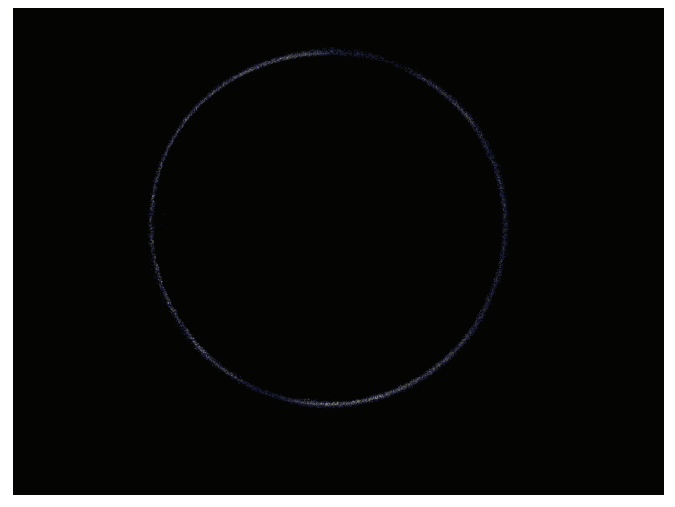

Figure 3. The collected image with the probe developed in this work.

\section{Conclusions}

To solve problems in measurement of translucent object, a camera embedded with a polarizer has been employed for the disk beam probe. It can minimize the stray light effectively. Same method can be applied to measurement of inner profile of the opaque objects with smooth surfaces. 


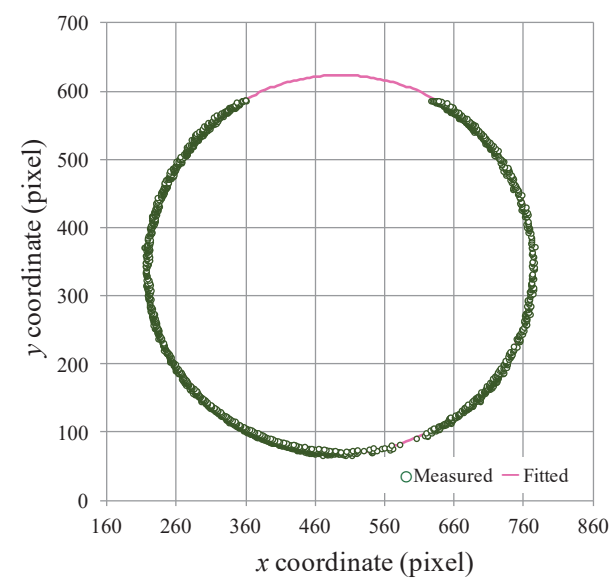

Figure 4. Inner profile (green round) of sample and the fitted results (pink line)

\section{References}

[1] Yoshizawa, T., Yamamoto, M., and Wakayama, T., "Inner profile measurement of pipes and holes using a ring beam device," Proc. SPIE 6382, 63820D (2006).

[2] Jin, L., Miyatsu, N., Kondoh, E., Gelloz, B., Kanazawa, N., and Yoshizawa, T., "Measurement of diameter of cylindrical openings using a disk beam probe," Optical Review 25, 656-662 (2018).

[3] Buschinelli, P., Pinto, T., Silva, F., Santos, J., and Albertazzi, A., "Laser Triangulation Profilometer for Inner Surface Inspection of 100 millimeters (4") Nominal Diameter,” J. Phys. Conf. Ser. 648, 012010 (2015).

[4] Tompkins, H. G., and Irene, E. A., [Handbook of Ellipsometry], William Andrew pulishing \& Springer, New York, 67-89 (2005). 
Role of zeroth-order diffraction beam and scattering light in three-dimensional shape measurement of fine structure by detecting phase distribution based on speckle interferometry

\section{Yasuhiko Arai (Kansai University)}

Generally, it is known well that an image-formation of the object which is beyond the diffraction limit of the objective lens in the observation of the fine structure by a microscope under the Abbe imaging theory is difficult. However, it is reported that the shape measurement method of a 3-D micro fine object, which is based on the measurement principle of speckle interferometry using a scattering light as the illumination light, can measure the shape by using the lateral shift of the measured object without any image formation process. In this paper, because speckle interferometry is originally the technology based on the use of the scattering light, the validity of using the scattering light as the illumination light is discussed in the new measurement method for the micro fine structure using speckle interferometry.

In the investigation, first, the diffraction grating whose section is a sawtooth is set exactly at the position of the focal point by the optical system using a plane wave. The period of the grating is $833 \mathrm{~nm}$. The first experimental condition is defined as using the plane wave, and, the surface of the object is focused on as possible as it can. Furthermore, the NA (numerical aperture) can be changed from 0.62 to 2.29 by inserting an aperture whose diameter is $8 \mathrm{~mm}$ in front of the objective lens in this optical system. When NA is changed, the value of the diffraction limit of the lens is also changed. Therefore, the image is focused on when the aperture is not set. On the other hand, the image formation is not made in the case of using the aperture. That is, the experimental condition for image formation of the object can be changed by inserting the aperture.

Additionally, the validity of using scattering light as the illumination light is discussed as the third condition by inserting the ground glass. In the case of setting no aperture in front of the objective lens, the speckle pattern which includes a fringe image can be grabbed. The three-dimensional shape which looks like a sawtooth is detected by analyzing such a speckle pattern. The aperture is set in front of the objective lens in the second experiment. Then, the image can be taken by virtually setting a situation beyond the diffraction limit under the condition of the second experiment. This image is taken in the condition of shutting out the higher-order diffraction lights and of passing only zeroth-order diffraction light through the aperture.

However, though the amplitude of the shape of the measured result is small, the information of the fringe image, which cannot be originally observed in the speckle pattern, 
can be taken as the shape of the object. That is, it is thought that the three-dimensional shape information also exists in the zeroth-order diffraction light.

Next, in the situation which the measured object is made image formation, the ground glass is inserted in order to change from the condition of the second experiment to that of the third experiment. The speckle pattern is taken under this condition. Since the condition of taking the image is beyond the diffraction limit of the objective lens, the fringe image cannot be observed in this image.

However, when the speckle pattern is analyzed, the shape which looks like a sawtooth can be detected. The image which makes image formation changes in the case of exceeding the diffraction limit of the objective lens because NA is changed from 0.62 to 0.29 . However, though the image formation cannot be performed, the sawtooth result is obtained from this analysis. In the case of using the scattering light as the illumination light under the condition of exceeding the diffraction limit of the lens, it means that much information concerning the deformation of the measured object from the zero-order diffraction beam can be detected by using the scattering light. On the other hand, in the case of using scattering light as the illumination light, the focused image cannot be taken as a speckle pattern. However, it can be thought that the situation which the phase information exists widely on the position around the focal point by using scattering light as illumination light happens. And, it can be also thought that the phase distribution information concerning the shape of the object can be recorded in a wide range on the optical axis by using scattering light which has the component of complexed directions as the illumination light.

However, it is confirmed that the use of scattering light is much useful than the case of using a plane light in the observation of the fine structure using speckle interferometry because the phase information exists in the zeroth-order diffraction light. In any case, it can be confirmed that the three-dimensional shape of the micro structure can be measured by the speckle interferometry using only the zeroth-order diffraction beam.

In this paper, the experiments concerning the phase change of only the zeroth-order diffraction light by the lateral shift of the object were performed by shutting out the higher harmonic diffraction lights to the objective lens set up the aperture in front of the lens. The possibility of three-dimensional shape measurement by the new method was confirmed by such experimental results. Furthermore, even if the image of the object cannot be focused, it is confirmed that the three-dimensional shape measurement using the objective whose diffraction limit is $523 \mathrm{~nm}$ can be performed by analyzing only the zeroth-order diffraction light in the case of the measurement of the diffraction grating whose period is $278 \mathrm{~nm}$. 


\title{
Numerical Analysis of Near-Field Light Intensity of Whispering Gallery Mode on Microsphere Surface with SNOM Probe
}

\author{
Bohuai Chu*1, Zheng Zhao ${ }^{3}$, Masaki Michihata², Kiyoshi Takamasu ${ }^{3}$, Satoru Takahashi \\ ${ }^{1}$ Department of Advanced Interdisciplinary Studies, The University of Tokyo, 4-6-1 Meguro-ku, \\ Tokyo, 153-8904, Japan \\ ${ }^{2}$ Research Center for Advanced Science and Technology, The University of Tokyo, 4-6-1 Meguro-ku, \\ Tokyo, 153-8904, Japan \\ ${ }^{3}$ Department of Precision Engineering, The University of Tokyo, 7-3-1 Bunkyo-ku, Tokyo, 113-8654, \\ Japan
}

\begin{abstract}
The accuracy of Micro-CMMs for three-dimensional shape measurement is influenced by the size and the shape of the probe sphere, which is usually about $100 \mu \mathrm{m}$ scale. In order to measure the diameter of those microspheres precisely, a new method based on whispering gallery mode (WGM) is proposed. In order to get the mode number of a WGM, which is necessary for diameter measurement, a method of using a scanning near field optical microscopy (SNOM) probe to measure the near-field light intensity of WGM on microsphere surface is proposed. Then in order to verify the disturbance of the SNOM probe on WGM resonance conditions, a numerical analysis was conducted. As a result, although the SNOM probe will cause the light intensity on the surface of the microsphere drop, the mode number can be correctly measured if the near-field light intensity can be detected sensitively.
\end{abstract}

Keywords: whispering gallery mode, SNOM probe, microsphere, diameter measurement

\section{Introduction}

Micro manufacturing technologies such as micro electro mechanical system (MEMS) have developed remarkably in the past several decades. In order to measure and evaluate the geometric shapes of micro parts like MEMS, micro three-dimensional coordinate measuring machine (Micro-CMM) is often used. In principle, the diameter of the probe sphere of MicroCMM is required for a measurement based on MicroCMM. Considering that those probe spheres are usually in the size of $100 \mu \mathrm{m}$ scale, and the required measurement accuracy is $50 \mathrm{~nm}$ or less, it is necessary to ensure that the diameter of the probe sphere of the Micro-CMM is measured with an accuracy of $10 \mathrm{~nm}$. In this case, making use of whispering gallery mode (WGM) resonance, an optical phenomenon which is discovered only within the sphere, a new measurement principle specialized for microsphere measurement is proposed ${ }^{[1]}$. In this microsphere measurement method, the mode number of

*hu@nanolab.t.u-tokyo.ac.jp; phone +81-03-5452-5183
WGM resonance is necessary. Until yet, this mode number was usually estimated by fitting with the theoretical value, which may lead to incorrect result. In order to measure the mode number directly, a method of detecting the near-field light intensity on the surface of a microsphere in WGM resonance condition by a scanning near field optical microscopy (SNOM) probe was proposed $^{[2]}$.

In this paper, in order to study the disturbance on the near-field light intensity distribution caused by the SNOM probe, a numerical analysis based on COMSOL Multiphysics was conducted.

\section{Measurement Principle}

1. Principle of Diameter Measurement Based on Whispering Gallery Mode

WGMs are the light propagation mode along the sphere surface. As shown in Figure 1 left, the light propagates along the equatorial line of the sphere in WGMs. When an integer multiple of the wavelength of the propagating light is equivalent to the circumference of a sphere, the 
light resonates, which is called WGM resonance. This integer multiplied is called mode number. If the resonant wavelength and the mode number is known, it is possible to calculate the length of circumference, that is, the diameter of the sphere.
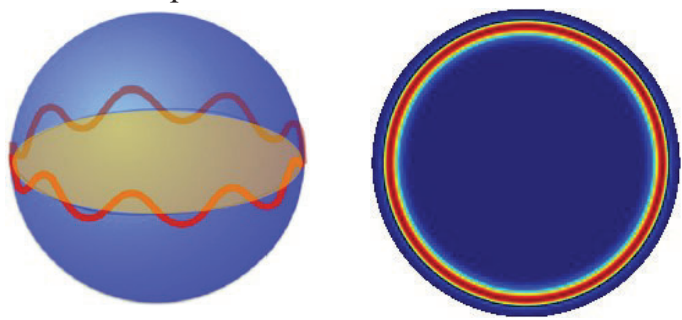

Figure 1. Schematic of WGM (left); Light intensity distribution of a traveling wave WGM resonance (right)

\section{Principle of Mode Number Measurement}

Next, the principle of mode number measurement is explained. In WGM resonance condition, according to traveling wave or standing wave, there are two states of light intensity distribution in the sphere. If the light is in traveling wave state, as shown in Figure 1 right, because of the light propagation is much faster than the response speed of detector available in actual experiment, the detectable intensity distribution will be uniform along the circumference. Conversely, if the light is in standing wave state, as shown in Figure 2, the intensity distribution is static, and the intensity changes periodically surround the circumference on the surface of the sphere. In addition, the light that distribute outside the surface of the

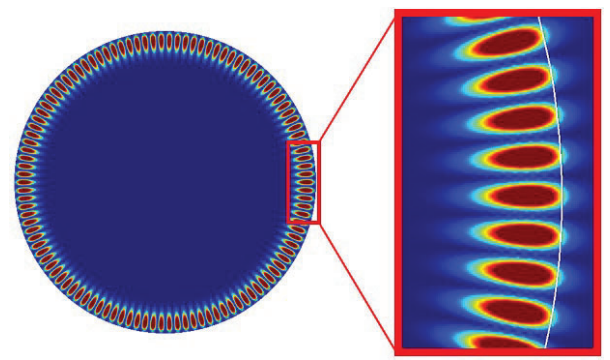

Figure 2. The light intensity distribution of WGM resonance. microsphere (near-field light) shows the same periodic variation, so that it is possible to detect the periodic variation of the light intensity by a SNOM probe, then, mode number can be obtained.

However, in order to detect the near-field light, the tip of SNOM probe must be put very close to the surface of microsphere (less than wavelength scale). So the probe may disturb the light intensity distribution.

\section{Numerical Analysis of Near-Field Light}

\section{Intensity distribution outside the microsphere}

In order to evaluate the near-field light intensity distribution disturbance caused by the SNOM probe, a numerical simulation was conducted. For the sake of computational complexity, the model was planar, and the SNOM probe was imagined as a pointed probe of uniform golden material. The probe is scanned at a distance of 50 $\mathrm{nm}$ near the surface of a circle in WGM resonance condition. The diameter of the circle was $20 \mu \mathrm{m}$, and the scan range of the probe was between $\pm 1 \mu \mathrm{m}$. Because the scan range is very small comparing with the circle diameter, the scan direction was set to vertical (from below to above, see Figure 3). In addition, the microsphere and the surrounding medium are set to glass $(\mathrm{n}=1.5013)$ and air $(\mathrm{n}=1)$, respectively, and the wavelength was set to $1549.48 \mathrm{~nm}$, which is a WGM resonance wavelength. According to Figure 3, the light intensity surround the circle surface changed evidently according to different probe positions. This phenomenon was due to the periodic interference of the probe to the resonance condition.

Next, the details of the disturbance of the near-field light intensity distribution caused by the probe is explaned. Figure 4 shows the maximum light intensity inside the circle when the probe was in different scanning positions. It is obvious that the maximum light intensity became
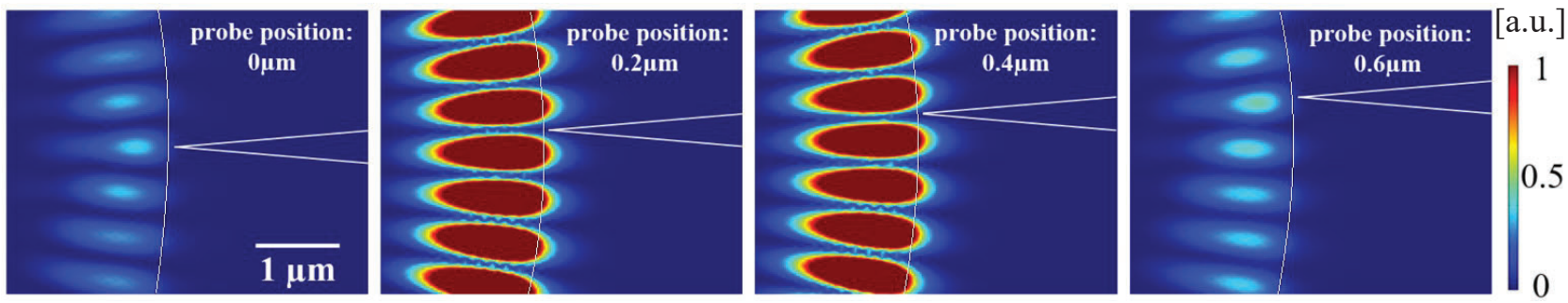

Figure 3. The disturbance to light intensity distribution caused by the probe in different probe position. 
lower when the probe pointed the strong light region directly. Otherwise, when the position of the probe was between two strong light regions, the maximum light intensity became larger. According to this result, when the probe scanned the circle surface in WGM resonance condition, whether the measured distribution can show similar tendency with the no-probe case become worthy of discussion.

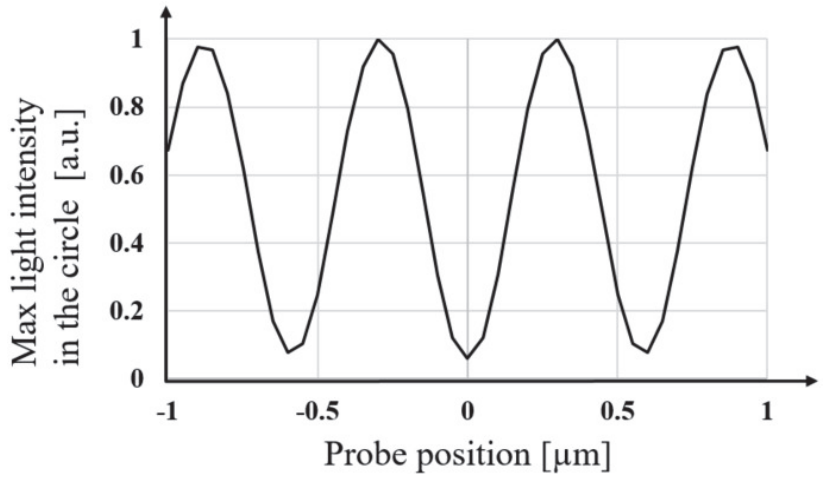

Figure 4. The periodic variation of maximum light intensity inside the circle according to probe position.

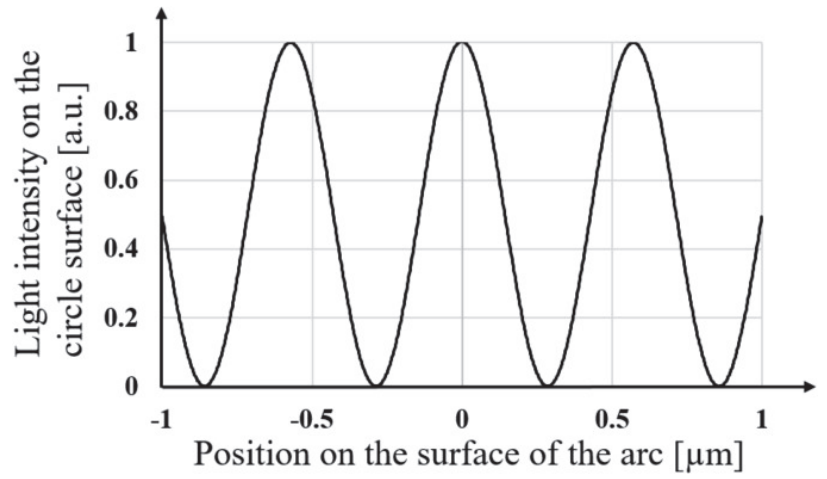

Figure 5. Light intensity on the circle surface in no-probe case.

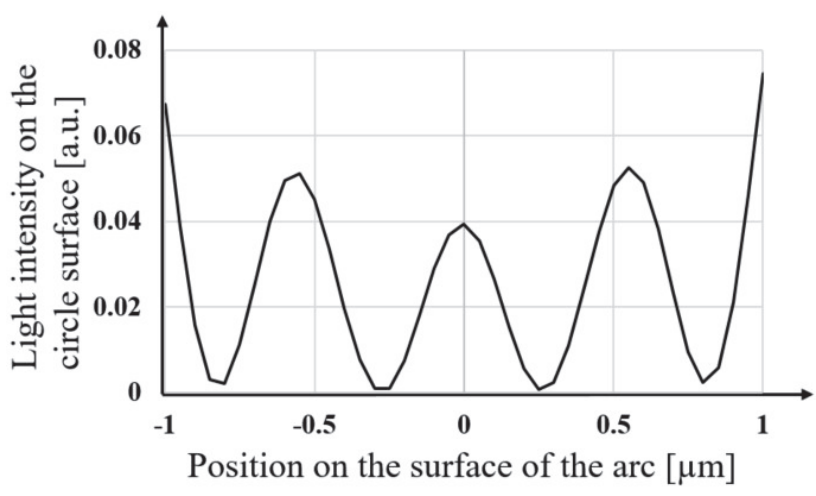

Figure 6. Light intensity on the circle surface when the probe exists.
Figure 5 shows the light intensity distribution on the circle surface in no-probe case, and Figure 6 shows the light intensity at some sampled positions on the circle surface when the probe was pointed to that position. As the results shown in Figure 5 and Figure 6, although when the probe existed, the light intensity on the circle surface is much lower than no-probe case, whether the probe exists or not, the distribution of the light intensity is similar. This result suggested that if the light intensity on the surface of the microsphere can be detected sensitively, even if the light intensity distribution is affected by the probe, the mode number can be measured correctly.

\section{Conclusion}

For measurement of microsphere diameter based on whispering gallery mode, the mode number of WGM resonance is required. In order to obtain the mode number, a method of measuring the near-field light intensity distribution on the surface of microsphere by SNOM probe was suggested. In this paper, a numerical analysis of WGM resonance in a simplified circle-and-probe system was conducted, and the result showed that the SNOM probe will cause the light intensity on the surface of the microsphere drop due to the disturbance to the WGM resonance condition, but it will not change the periodic distribution of light intensity. Therefore, it is possible to measure the mode number by a SNOM probe.

\section{Acknowledgement}

This work was financially supported by Foundation for Interaction in Science \& Technology, Mizuho Foundation for the Promotion of Sciences, A-STEP from JST, and MEXT/JSPS KAKENHI (No. 18K18803, 15H05505).

\section{References}

[1] Michihata, M., et al., "Measurement of Probe-stylus Sphere Diameter for Micro-CMM Based on Spectral Fingerprint of Whispering Gallery Modes", CIRP Annals, Vol.63, Issue 1, pp.469-472 (2014).

[2] Götzinger. S., et al., "Mapping and manipulating whispering gallery modes of a microsphere resonator with a near-field probe", Journal of Microscopy, Vol. 202, Pt 1, pp. 117-121 (2001). 


\title{
Light attenuation in the bistatic scattering measurement in the atmosphere
}

\author{
Yuzhao Ma*, Huiliang Gao, Ruisong Wang, Xinglong Xiong \\ Tianjin Key Laboratory for Advanced Signal Processing, Civil Aviation University of China, Tianjin \\ 300300, P.R. China
}

\begin{abstract}
In this paper, we for the first time investigate the light attenuation in the bistatic scattering measurement with MonteCarlo method using the actual aerosol data in Beijing area in China.
\end{abstract}

Keywords: light attenuation, bistatic, scattering measurement, aerosol extinction coefficient

\section{Introduction}

The bistatic scattering measurement has a broad application background in recent years. For example, the scattered solar-blind ultraviolet (UV) light can be used in free-space optical communication to provide a non-lineof-sight (NLOS) link when the line-of-sight (LOS) link between transmitter and receiver is blocked. The light attenuation in the bistatic scattering measurement has been discussed recently. Most of the literatures focused on the effects of the measurement system structure on the light attenuations through the atmosphere. However, aerosol particles existed in the link of the measurement system also have important influence on the measurement. In the paper we for the first time investigate the light attenuation in the bistatic scattering measurement using the actual aerosol data in Beijing area in China. We relate the light attenuation with aerosol extinction coefficient and asymmetry factor.

\section{Single Scattering Characteristics of}

\section{Atmospheric Aerosol}

The extinction coefficient and asymmetry factor are the two important optical properties of aerosol particles. Aerosol extinction coefficient is used for characterizing the ability of scattering and absorbing light of aerosol particles. And asymmetry factor represents a preferred direction in scattering. The value is 1 if the scattering is completely in the forward direction and -1 for completely backward scattering. Otherwise, visibility, known as meteorological optical range, also depends on aerosol extinction coefficient, and low visibility may affect the bistatic scattering measurement. Therefore, we consider to choose the aerosol data in low visibility days for investigating the effects of aerosol extinction coefficient on the light attenuation in the bistatic scattering measurement. In this paper, we choose four days in 2018 in Beijing area and collect the microphysical and optical properties of atmospheric aerosol of these four days from AERONET as data samples. After investigating the visibility of the whole spring in 2018 according to the data from China Meteorological Administration, we find that there are four days with quite low visibility, with corresponding atmospheric aerosol extinction coefficient values of 1.83, 2.39, 3.69 and 3.40, respectively, with unit of $\mathrm{km}^{-1}$. Those are the four days we have chosen.

The aerosol particles in real atmosphere exist in the form of a group of particles with different sizes and optical properties. Therefore, for the bistatic scattering measurement in real atmosphere, atmospheric aerosol extinction coefficient should be defined as the total extinction cross section of all particles with different sizes in a unit volume, in the form of normalized extinction coefficient, as follows.

$$
\sigma=\int_{r_{\min }}^{r_{\max }} C_{\text {ext }}(r) n(r) d r
$$

in which $\sigma$ is aerosol extinction coefficient, $C_{e x t}$ is aerosol extinction cross section and $n(r)$ is the size distribution of atmospheric aerosol particle .

Similarly, the asymmetry factor of real atmosphere should also be defined as the normalized asymmetry factor of all asymmetry factors of all particles with different size distributions and optical properties. Here we 
give the normalized asymmetry factor:

$$
g=\frac{\int_{r_{\min }}^{r_{\max }} n(r) C_{e x t}(r) g(r) d r}{\int_{r_{\min }}^{r_{\max }} n(r) C_{e x t}(r) d r}
$$

\section{Light Attenuation of Bistatic Scattering \\ Measurement}

In this part, we build a bistatic scattering measurement system model and the system geometry is depicted in Fig. 1. The demonstrations and the corresponding values of the system parameters are shown in Table 1.

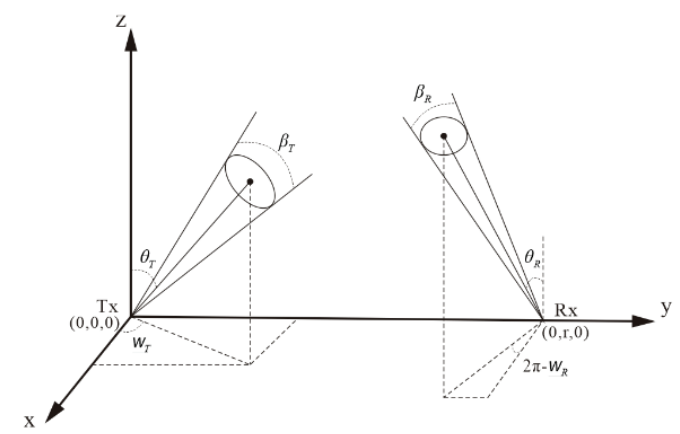

Figure 1. Structure of bistatic scattering communication system

Table 1. Demonstration and values of the system parameters.

\begin{tabular}{|c|c|c|}
\hline Parameter & Demonstration & value \\
\hline$\theta_{T}$ & $\begin{array}{c}\text { the inclination angle } \\
\text { of the transmitter }\end{array}$ & $30^{\circ}, 60^{\circ}$ \\
\hline$\phi_{T}$ & $\begin{array}{c}\text { the azimuth angle } \\
\text { of the transmitter }\end{array}$ & $90^{\circ}$ \\
\hline$\theta_{R}$ & $\begin{array}{c}\text { the inclination angle } \\
\text { of the receiver }\end{array}$ & $30^{\circ}, 60^{\circ}$ \\
\hline$\phi_{R}$ & $\begin{array}{c}\text { the azimuth angle } \\
\text { of the receiver }\end{array}$ & $270^{\circ}$ \\
\hline$\beta_{T}$ & $\begin{array}{c}\text { The beam width of } \\
\text { the transmitter }\end{array}$ & $10^{\circ}$ \\
\hline$\beta_{R}$ & $\begin{array}{c}\text { The beam width of } \\
\text { the receiver }\end{array}$ & $30^{\circ}$ \\
\hline
\end{tabular}

Firstly, we analyze the effects of aerosol optical properties mentioned in the above section on the bistatic scattering measurement. The changes of light attenuation with aerosol asymmetry factor and extinction coefficient are shown in Fig. 2 and Fig. 3. We have considered different system structure. Fig. 2 are the results in assuming the inclination angle of transmitter and receiver are $\left(30^{\circ}, 30^{\circ}\right)$ and Fig. 3 for $\left(60^{\circ}, 60^{\circ}\right)$.

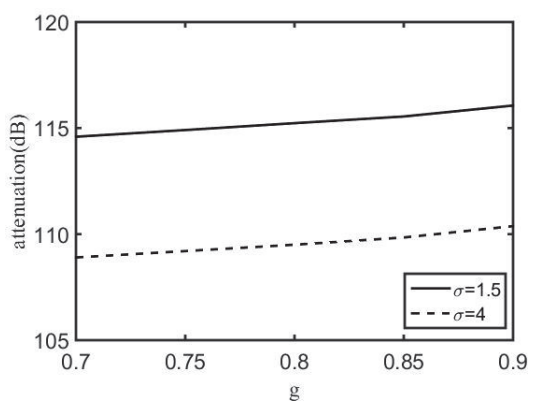

Figure 2(a). Light attenuation for different aerosol extinction coefficient for the system angle pair of $\left(30^{\circ}\right.$, $30^{\circ}$ ) and measurement range $r=50 \mathrm{~m}$

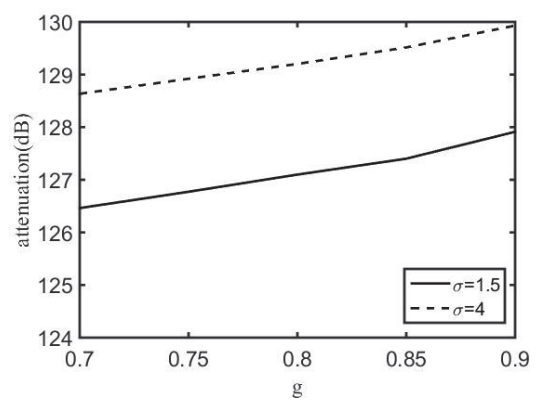

Figure 2(b). Light attenuation for different aerosol extinction coefficient for the angle pair of $\left(30^{\circ}, 30^{\circ}\right)$ and measurement range $r=200 \mathrm{~m}$

As shown in Fig. 2(a) and Fig. 2(b), the light attenuation increases along with aerosol asymmetry factor. And the light attenuation of long-range measurement is much higher than that of short-range measurement. Notably, for short-range measurement $(r=50 m)$, higher aerosol extinction coefficient leads to higher light attenuation and for long-range measurement $(r=200 m)$, higher aerosol extinction coefficient leads to lower light attenuation. This is because for short-range measurement, when the aerosol extinction coefficient is low, the photons from the transmitter have lower probability to be scattered by aerosol particles and thus cannot be scattered towards the receiver. However, for the long-range measurement, when the aerosol extinction coefficient is quite high, the 
photons from the transmitter may be scattered too much by aerosol particles and then be scattered away from the receiver.

In Fig. 3(a) and Fig. 3(b), we analyze the condition of another pair of the inclination angle of transmitter and receiver. In this condition, we can also obtain the conclusion proposed above. Specially, comparing Fig. 3 with Fig. 2, we find that when the inclination angles of transmitter and receiver are larger, the light attenuation of the bistatic scattering measurement system becomes lower.

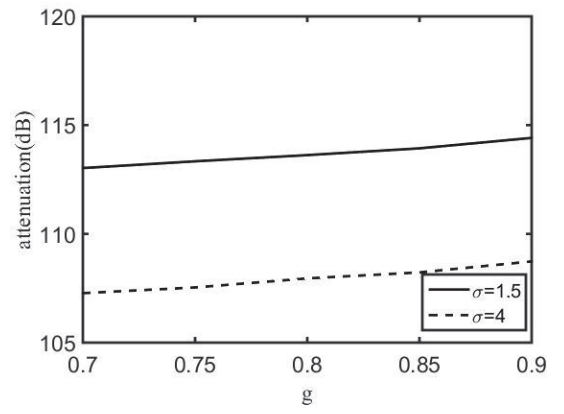

Figure 3(a). Light attenuation for different aerosol extinction coefficient for the angle pair of $\left(60^{\circ}, 60^{\circ}\right)$ and measurement range $r=50 m$

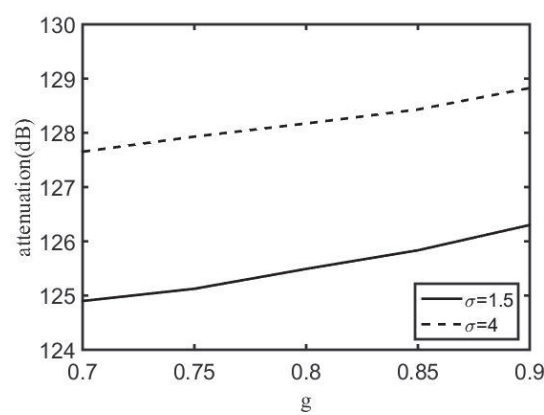

Figure 3(b). Light attenuation for different aerosol extinction coefficient for the angle pair of $\left(60^{\circ}, 60^{\circ}\right)$ and measurement range $r=200 \mathrm{~m}$

Then we investigate the light attenuation of the system for the given four days we have chosen. As shown in Fig. 4(a) and Fig. 4(b), the light attenuation of the system increases along with the measurement range. Similarly with the conclusion we obtained above, when the measurement range is shorter than $150 \mathrm{~m}$, the higher aerosol extinction coefficient leads to lower light attenuation and for the measurement range longer than $150 \mathrm{~m}$, the higher aerosol extinction coefficient leads to higher light attenuation.

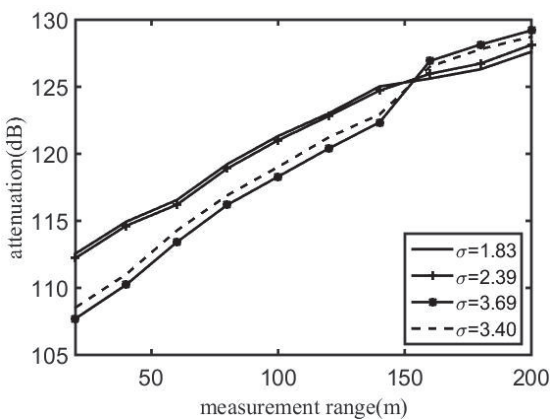

Figure 2(a). Light attenuation for the given four days for the angle pair of $\left(30^{\circ}, 30^{\circ}\right)$

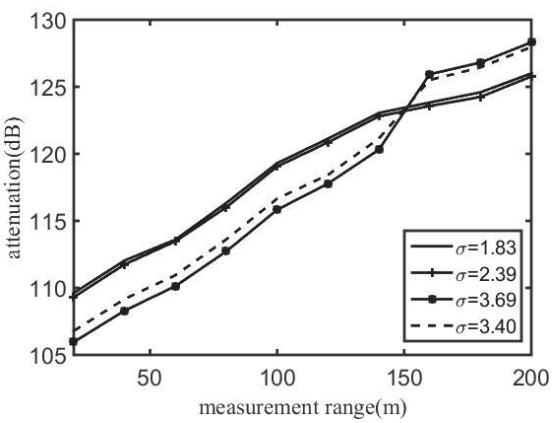

Figure 2(b). Light attenuation for the given four days for the angle pair of $\left(60^{\circ}, 60^{\circ}\right)$

In conclusion, in the bistatic scattering measurement system, an increase of atmospheric asymmetry factor and measurement range strengthens the light attenuation. And specially, for short-range measurement, higher aerosol extinction coefficient can reduce the light attenuation and for long-range measurement, the light attenuation will definitely increase with aerosol extinction coefficient.

\section{References}

Ding, H., Chen, G., Majumdar, A. K., Sadler, B. M. and $\mathrm{Xu}, \mathrm{Z}$. Y. 2009. Modeling of non-line-of-sight ultraviolet scattering channels for communication. IEEE Journal on Selected Areas in Communications 27(9):1535-1544.

Drost, R. J., Moore, T. J. and Sadler, B. M. 2011. UV communications channel modeling incorporating multiple scattering interactions. Journal of the Optical Society of America Optics Image Science 28(4):686.

Xu, C., Zhang, H. and Cheng, J. 2015. Effects of haze particles and fog droplets on NLOS ultraviolet communication channels. Optics Express 23(18): 23259-23269. 


\title{
Flyable Mirrors: Laser Scanning Vibrometry Method for Monitoring Large Engineering Structures Using Drones
}

\author{
Mohamed A. A. Ismail ${ }^{* a}$, Andreas Bierig ${ }^{\mathrm{a}}$, Saher R. Hassan ${ }^{\mathrm{b}}$, Rolf Kumme ${ }^{\mathrm{b}}$ \\ ${ }^{\text {a} D L R ~(G e r m a n ~ A e r o s p a c e ~ C e n t e r), ~ I n s t i t u t e ~ o f ~ F l i g h t ~ S y s t e m s, ~ L i l i e n t h a l p l a t z ~ 7, ~} 38108$ \\ Braunschweig, Germany \\ ${ }^{b}$ Physikalisch-Technische Bundesanstalt, Bundesallee 100, 38116 Braunschweig, Germany
}

\begin{abstract}
Laser Doppler vibrometers (LDVs) provide an efficient method for measuring the surface vibration of a remote target using the Doppler shift of a reflected laser beam. However, LDVs have limited applicability for the evaluation of large structures, e.g. bridges and wind turbine towers. In this study, a new scanning method utilizing flyable mirrors is introduced to enable a single LDV unit to scan a large structure via reflection of the laser beam from mirrors attached to drones. Preliminary tests have been performed to measure structural vibrations between 2 and $900 \mathrm{~Hz}$ through an LDV and a flyable mirror attached to a micro drone.
\end{abstract}

Keywords: laser vibrometry, micro drones, reflective mirrors, structure health monitoring, vibration analysis.

\section{Introduction}

Structural vibration analysis is used to verify the safety and reliability requirements of engineering structures and to provide early detection of mechanical failures [1]. For large engineering structures such as bridges, a common measurement practice is to mount tens to hundreds of accelerometers at critical points on the structure [2].

In addition to the high cost of this practice, the service reliability of accelerometers cannot match the nominal service-life of bridges without the costly replacements [2].

One of the alternatives for vibration monitoring of bridges is long-range laser Doppler vibrometry (LDV) [3]. LDVs provide non-contact measurement of remote surfaces by measuring the Doppler frequency shift of a reflected laser beam due to the motion of a target surface.

However, LDVs have in principle a limited applicability for large structures, because laser beams should be applied perpendicularly to target surfaces. Consequently, tilted surfaces need more complex setups (multiple LDVs or robotic LDVs) which can be used for more compact targets (e.g. cars [4]), but not for much larger structures. Therefore this work introduces a new method that extends the scanning applicability of LDVs to large structures in order to support structure health monitoring of critical engineering assets.

\section{Flyable Mirrors Method}

The method introduced here measures vibration of large structures using an LDV and one or more reflective mirrors attached to drones [5], as shown in Figure 1.
The LDV is mounted on a motorized motion stage, allowing adjustment of tilt and rotation angles by a central control unit. The reflective mirrors on the drones guide the laser beams from the LDV to scan a specific area of a large structure. The primary benefit of the method is its costefficiency, as structures can be scanned using a single LDV and a drone. Flyable mirrors are typically thin, light sheets that require only low-cost drones with payload capacities of a few hundred grams. The operating cost is significantly low thanks to recent advances in autonomous drone navigation and control. A key safety advantage of the method is that micro-drones have limited collision risk in case of sudden failure compared to a heavy drone carrying a complete LDV.

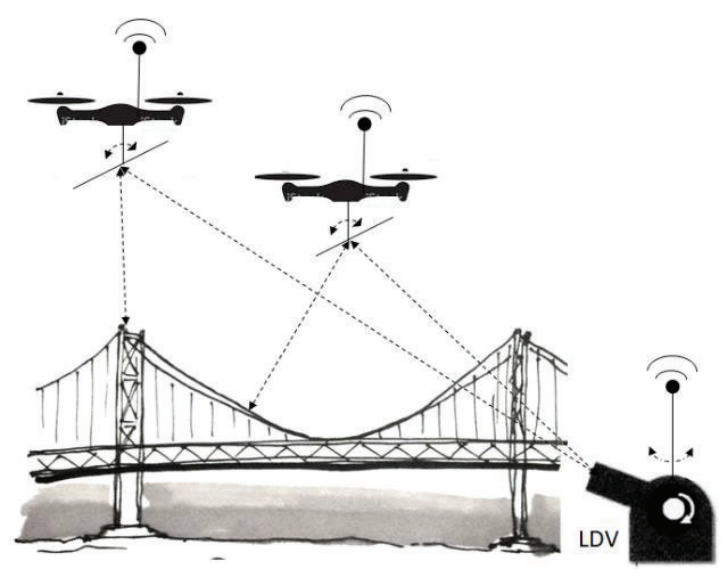

Figure 1. The principle of using reflective mirrors attached to micro-drones to increase accessible LDV scanning of large engineering structures [5] 
Qualifying the flyable mirror method for practical measurement applications requires investigation of several challenges:

- Influence of internal vibration noise generated by the drone's propellers on measurement quality.

- Positioning stability and accuracy of drones carrying flyable mirrors.

- Measurement performance for different ambient conditions, geometric arrangements, and distances.

This paper presents initial investigations of the first challenge, the influence of the drone's vibration noise. Measurements were performed in laboratory conditions using a mirror fixed to a quadcopter and a vibration target represented by an electro-mechanical shaker.

\section{Experimental Setup}

A key challenge to the flyable mirror method is the efficiency with which the target vibration can be separated from the internal vibration noise generated by the drone's propellers. A simple experiment is considered, as shown in Figures 2 and 3. A vibration target is created on top of an electro-mechanical shaker by feeding sinusoidal signals using an arbitrary generator into a power amplifier, which drives the coils of the shaker armature. The LDV is used to measure the vibration through two paths in series. Path 1 is a reference path with one fixed mirror (part of the test stand) between the LDV and the shaker, while Path 2 additionally includes the mirror attached to the microdrone (a quadcopter model AR Parrot 2) hovering above the shaker.

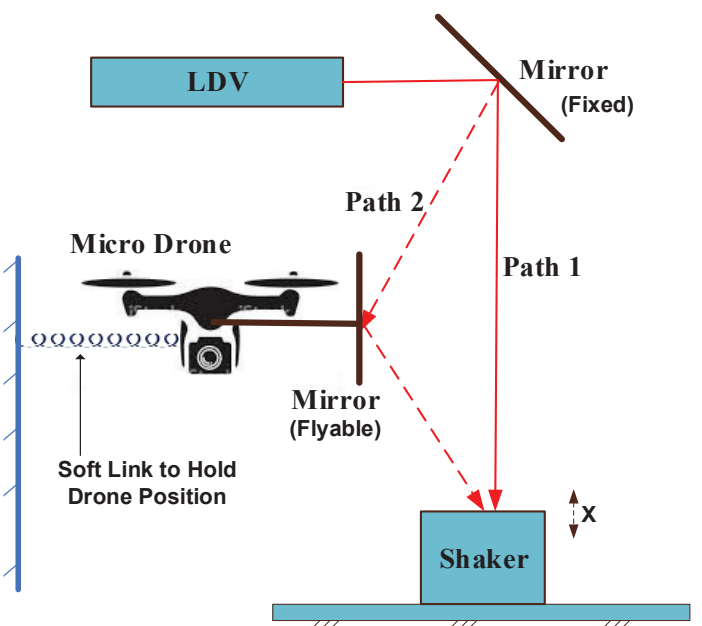

Figure 2. Schematic of experimental arrangement, the fixed mirror is a part of the LDV test stand

The experiment uses an LDV model PSV-400 from Polytec [4]. The frame of the LDV is equipped with a passive and active damping table to mechanically isolate the LDV from the shaker excitation and any sther
A soft link is added between the drone's boom and a stationary point to constrain the drone's position without attenuating the vibration of the propellers. The vibrations measured through Paths 1 and 2 are examined for two groups of target vibrations of the shaker: the low-frequency group of $(2,5,9) \mathrm{Hz}$ represents common resonance frequencies for large bridges [2, 3], while the highfrequency group of $(200,500,900) \mathrm{Hz}$ represents common rotating harmonics from gearboxes in wind turbines. The sampling frequencies are $1.2 \mathrm{kHz}$ (minimum LDV sampling rate) and $12.8 \mathrm{kHz}$ (available LDV rate higher than 10 times the target frequency) for low and high frequencies, respectively.
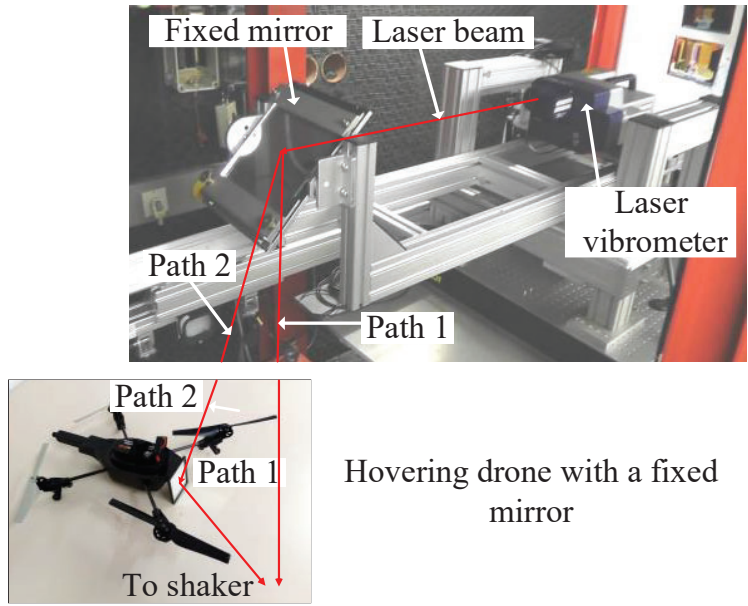

Hovering drone with a fixed mirror

Figure 3. Experimental setup for measuring remote vibrations using an LDV and flyable mirror method

\section{Results}

Figure 4 shows the fast Fourier transform (FFT) magnitudes of the high-frequency group for the two paths. A nearly constant frequency-independent attenuation is observed between the reference path (Path 1) and the flyable mirror path (Path 2).

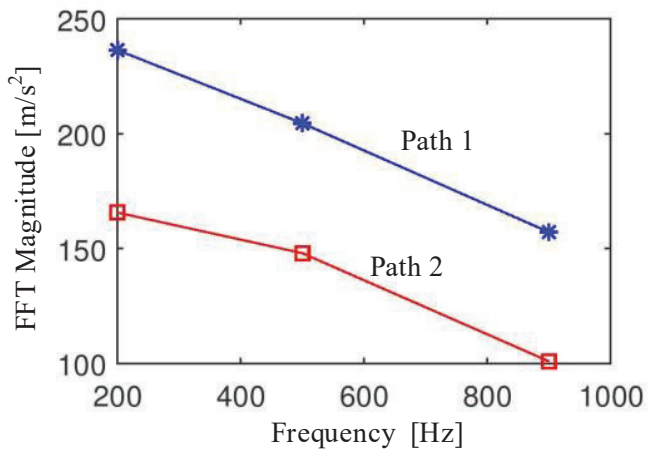

Figure 4. FFT magnitudes for high vibration frequencies measured through Path 1 and Path 2 
angle to the shaker. These factors will be thoroughly investigated in future experiments.

Figure 5 shows the FFT magnitudes of the lowfrequency group for the two paths at which irregular attenuation is observed. The drone is shaking around its position with a frequency near $2 \mathrm{~Hz}$. Such drone's activity causes additional energy to the overall FFT spectrum of the LDV's measured signal as the shaker frequency approaches drone's shaking frequency.

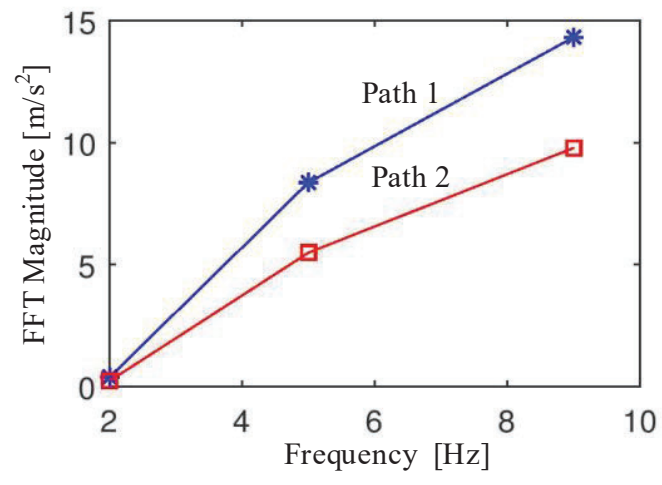

Figure 5. FFT magnitudes for low vibration frequencies measured through Path 1 and Path 2

Time waveforms of the $900 \mathrm{~Hz}$ signal are shown in Figure 6; signals for Path 1 and Path 2 are in phase. High frequency disturbances to the Path 2 signal, between 8 and 10 seconds, are a result of weak high frequency harmonics of drone's noise at multiple BPFs . In addition, a regular attenuation of the signal's amplitude is induced which matches magnitude attention in Figure 4 due to the incident angle and the imperfect reflectivity of drone's mirror.

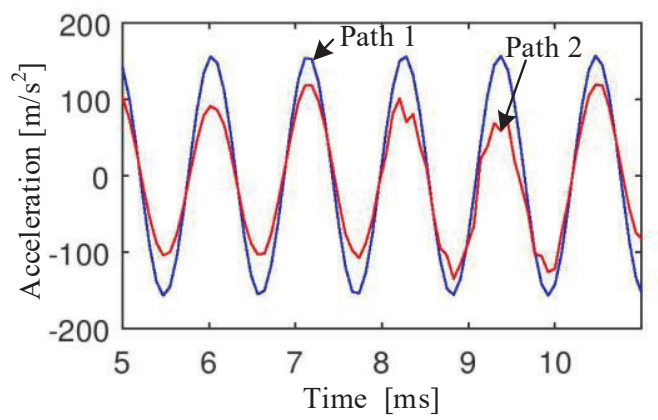

Figure 6. Time waveform examples for $900 \mathrm{~Hz}$ vibration signal measured through Path 1 and Path 2

For demonstrating the results at a single target frequency, FFT spectrums for Path 1 and Path 2 are depicted in Figure 7. The drone blade pass fipeodedisPIE Vol. 11 disturbang69-gaused by low frequency shaking of the drone. acoustic noise), which is observable in Path 2 in Figure 7. Because the noise of the drone can be predicted in advance based on the propeller speed, a low pass or band pass filter can be used to routinely filter out this noise. Thus except for the narrow bandwidth filtered out around BPF, the full spectrum is available. These results support the potential use of flyable mirrors for low and high frequency applications.
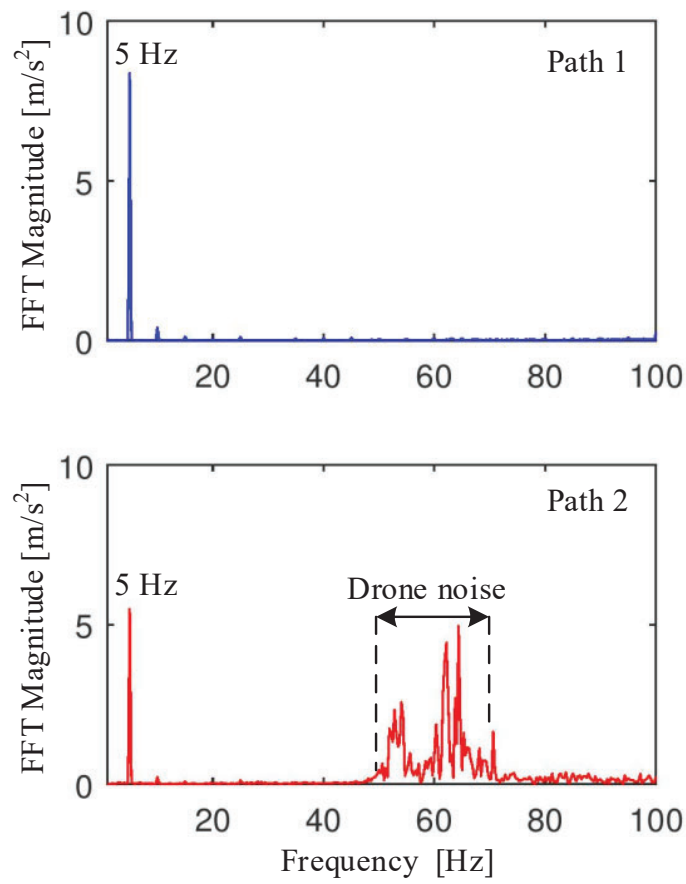

Figure 7. FFT spectra for vibration signals measured through Path 1 and Path 2 for a $5 \mathrm{~Hz}$ vibration signal

\section{Conclusions}

The flyable mirror method, comprising LDV measurements using a mirror attached to a drone, enables cost-effective vibration monitoring of large engineering structures. Several challenges have been identified to ensure the method is sufficiently mature for application in realistic conditions. A crucial challenge is related to the vibration noise from the drone's propellers, which could limit the applicability of the flyable mirror method. A basic experiment was conducted to study the influence of this noise source on the measured signal of a shaker set to vibrate at different frequencies: 2, 5, 9, 200, 500, and $900 \mathrm{~Hz}$. The drone noise was found to match the blade pass frequency (BPF) around $60 \mathrm{~Hz}$. Vibration measurements that are higher than the BPF were attenuated by a nearly constant factor due to the incident angle and the imperfect reflectivity of the flyable mirror. In contrast, measurements below the BPF were additionally subjected to significant 
mirror and broadening the frequency range of structural vibrations are planned for future work.

\section{References}

[1] Fritzen C. P., "Vibration-based structural health monitoring-concepts and applications," Key Engineering Materials. Trans Tech Publications. 293, 3-20 (2005).

[2] Inaudi, D., " Overview of 40 bridge structural health monitoring projects," Proc. International Bridge Conference IBC, 09-45 (2010).

[3] Miyashita, T., and Nagai, M., "Vibration-based structural health monitoring for bridges using laser Doppler vibrometers and MEMS-based technologies," Int. J. Steel Struct, 8(4), 325-331. (2008).

[4] Polytec GmbH, N., "RoboVib - structural test station for automated experimental modal testing," $<$ https://www.polytec.com/eu/vibrometry/products/fullfield-vibrometers/robovib/> (15 December 2018).

[5] Ismail, M. A. A., "DE102018113244: Verfahren und Vorrichtung zum Messen von Schwingungen eines Objekts unter Verwendung einer Drohne," German Patent Pending Application (2018). 


\title{
Fabrication of three dimensional nano-periodic structure by the Talbot lithography using multiple exposure*
}

\author{
Hiroki Nakanishi*a, Yasuhiro Mizutani ${ }^{\mathrm{a}}$, Yasuhiro Takaya ${ }^{\mathrm{a}}$

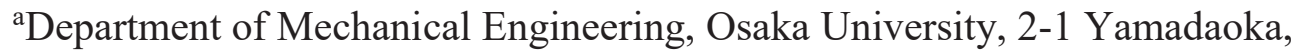 \\ Suita, Osaka, 565-0871, Japan
}

\begin{abstract}
Recently, there has been considerable interest in large area fabrication methods of three dimensional nano-periodic structures, such as photonic crystals and biomimetic structures. The Talbot lithography is known as a high efficient fabrication method using periodic light intensity distribution. However, because of difficulty to fabricate a three-dimensional structure, processing flexibility is low. The purpose of this study is to develop a highly efficient and flexible fabrication method using the Talbot lithography. In the proposed method, the three-dimensional light intensity distribution is generated by grating rotation and multiple exposure. In the experiment, a structure having three dimensional period of wavelength order was fabricated in the area of millimeter order. Hence, the proposed method is promising as a high efficient and flexible fabrication method of three dimensional nano-periodic structure.
\end{abstract}

Keywords: Talbot effect, lithography, three dimensional nano-periodic structure, multiple exposure.

\section{Introduction}

Recently, the interest in three dimensional nano-periodic structures has been stimulated by useful functions, such as photonic crystals. Machining methods into a large area is more desirable for a highly efficient fabrication. It is also necessary to control the period of the structure flexibly.

For high machining efficiency and flexibility, we propose three dimensional lithography process by the combination of the Talbot effect and multiple exposure. The Talbot effect has a three dimensional periodic light intensity distribution generated by light transmitted through the diffraction grating. Applied this phenomenon to a lithography, the periodic structure can be fabricated into a large area ${ }^{[1]}$. In the proposed method, a three dimensional periodic structure can be fabricated by rotating a grating and performing multiple exposure. The fabricated structure can be controlled by the pitch, rotation angle and position of the grating. In this paper, we performed multiple exposure using a grating with a period of wavelength order. As a result, we succeeded in fabricating a three dimensional periodic structure in the area of millimeter order.

\section{Fabrication method of three dimensional periodic structure by the Talbot lithography \\ 1. Principle of the Talbot lithography using multiple exposure}

Figure 1 shows a schematic diagram of the fabrication method of a three dimensional nano-periodic structure. We focused on a coplanar rotation of the grating to perform multiple exposure that superimposes light intensity distributions. As shown in fig. 1(a), after the grating is irradiated with UV light, periodic light intensity distribution called the Talbot effect is generated in the photoresist. The Talbot distance $z_{T}$, which is the period in the vertical direction, is given by

$z_{T}=2 d^{2} / \lambda$

Here, $d$ is the pitch of the grating and $\lambda$ is the wavelength of the light source. Subsequently, as shown in fig. 1(b), second exposure is performed after rotating the grating by an arbitrary angle $\theta$. As a result, three dimensional periodic structure can be fabricated by superimposing light intensity distributions in two exposures. The period of the structure can be controlled by the wavelength, grating pitch, rotation angle and position; that is, this method has high flexibility and machining efficiency. (a)

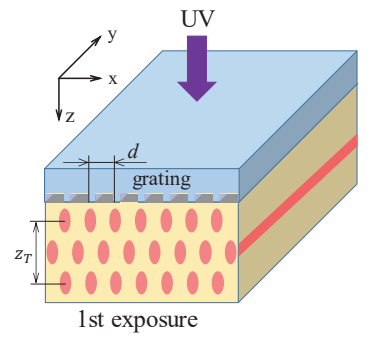

(b)

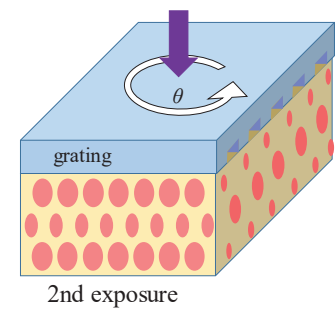

Fig. 1 Principle of fabrication of three dimensional periodic structure. (a) The Talbot effect and (b) three dimensionalization of structure by multiple exposure. 


\section{Fabrication process of nano-periodic structure}

Figure 2 shows the fabrication process of nano-periodic structure by the Talbot effect. In the Talbot lithography, the structure was fabricated by six processes. First, as shown in fig. 2(a), a photoresist was spin-coated on a substrate to form a film. Next, as shown in fig. 2(b), the sample was pre-baked using a hot plate for the solvent volatilization. Subsequently, as shown in fig. 2(c), a grating was placed on the photoresist, then exposed by UV light. The pitch of the diffraction grating was $750 \mathrm{~nm}$. In the case of multiple exposure, after the grating was rotated by $\theta$, the sample was exposed again. After exposure, as shown in fig. 5(d), post-baking was performed to complete the photosensitive reaction. Then, as shown in fig. 5(e), the photoresist was developed. Finally, as shown in fig. 5(f), the sample was rinsed. In this experiment, SU-8 3010 was used as a negative photoresist. (a)

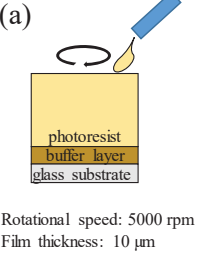

(d)

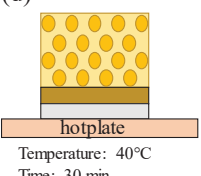

(b)

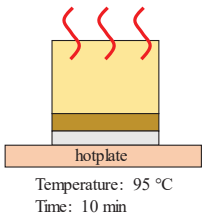

(e)

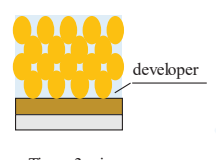

(c)

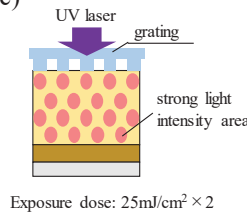

(f)

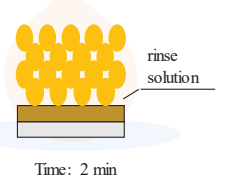

Fig. 2 Procedure of the Talbot lithography. (a) Spincoat, (b) pre-baking, (c) exposure, (d) post exposure bake, (e) development and (f) rinsing.

\section{Fabrication result of periodic structure}

Nano-periodic structures were fabricated by the lithography using the Talbot effect and multiple exposure. Table 1 shows the fabrication results of nano-periodic structures. First, in the single exposure process, observing from the cross section A, a structure in which a periodic light intensity distribution of the Talbot effect was transferred was fabricated. The period in the horizontal direction was $760 \mathrm{~nm}$, which almost corresponded to the theoretical value of $750 \mathrm{~nm}$. The period in the vertical direction was $3.57 \mu \mathrm{m}$. It is assumed that the difference from the theoretical value of $5.22 \mu \mathrm{m}$ was attributed to shrinkage during drying after rinsing. In addition, observing the structure from the cross section B (perpendicular to A), holes were progressing in the horizontal direction. From the above result, the usefulness of the Talbot lithography using single exposure was indicated.

Next, we attempted to fabricate three-dimensional structure by rotating the grating by $90^{\circ}$ and two exposures. Observing the surface of the structure, a two-dimensional grating structure was fabricated in the area of millimeter order. Observing from the cross section A, a structure having a period in two directions was observed. Observing the structure from a cross section B, same structure was also fabricated. In both cross sections, the period in the horizontal direction was $759 \mathrm{~nm}$, and the period in the vertical direction was $2.61 \mu \mathrm{m}$. This value corresponded to the theoretical value on the same order. From the above results, it was shown that a three dimensional nano-periodic structure was fabricated by the Talbot lithography using multiple exposure. Hence, this method enable fabrication with high processing efficiency and flexibility.

Table 1 Fabrication results of periodic structure by the

Talbot lithography using single and multiple exposure.

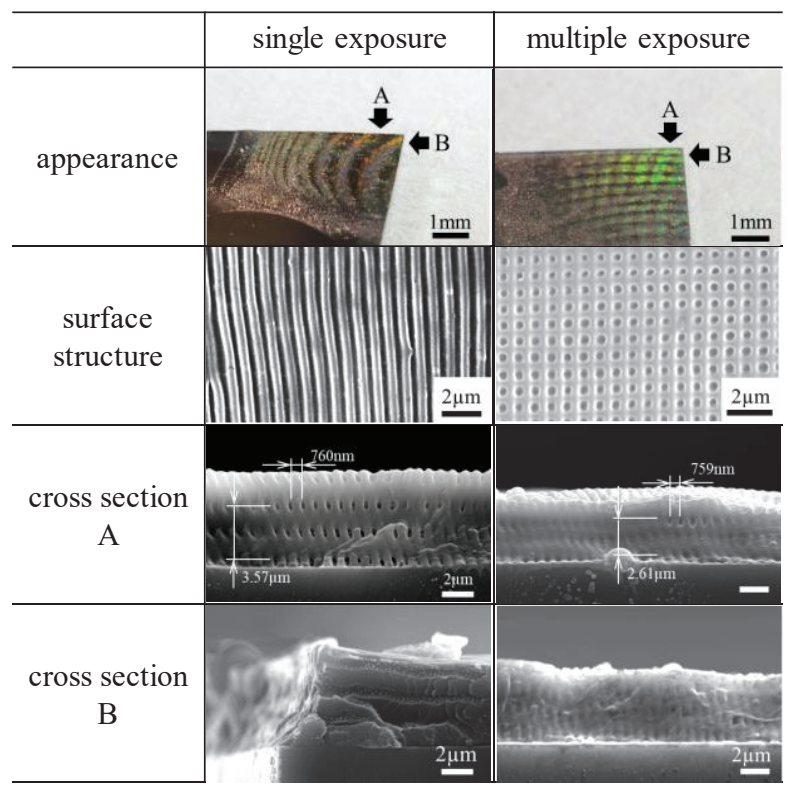

\section{Conclusion}

We provided a three dimensional lithography process by combination of the Talbot effect and a multiple exposure. The three dimensional structure with the period of wavelength order was fabricated in the area of millimeter order. Therefore, the proposed method is promising for fabrication method with high efficiency and flexibility.

\section{References}

[1] Seok. Jeon, et al.: "Fabricating complex three-dimensional nanostructures with high-resolution conformable phase masks," PNAS, 101, 34, (2004). 


\title{
Optical Trapping of Airborne Droplet for Laser Fabrication of 3-Dimensional
}

\section{Structure based on Optical Potential using Radially Polarized Beam}

\author{
Makoto YOKEI $^{\mathrm{a}^{*}}$, Masahiro HAYASHI ${ }^{\mathrm{a}}$, Masaki MICHIHATA ${ }^{\mathrm{b}}$, Kiyoshi TAKAMASU $^{\mathrm{b}}$ \\ and Satoru TAKAHASHI ${ }^{\mathrm{b}}$
}

\author{
${ }^{\mathrm{a}}$ The University of Tokyo, Department of Precision Engineering 7-3-1 Bunkyo-ku, Tokyo, 113-8654, \\ Japan \\ ${ }^{\mathrm{b}}$ The University of Tokyo, Research Center for Advanced Science and Technology, 4-6-1 Meguro-ku, \\ Tokyo, 153-8904, Japan
}

\begin{abstract}
Processing technology for manufacturing fine devices and next-generation functional parts are required. In this study, by focusing on the optical trapping potential due to the electric field intensity gradient, we aimed to process three-dimensional microstructures in a range smaller than $100 \mathrm{~nm}$ by localizing and three-dimensionally accumulating nanoparticles without being limited by the diffraction limit. In this paper, we aim to improve the optical trapping efficiency which is important in processing speed of three-dimensional microstructure by utilizing the fact that the component in the direction of the optical axis of the electric field becomes remarkably strong near the focal point of the radially polarized focused beam.
\end{abstract}

Keywords: Additive manufacturing, Brownian motion, Nanostructures, Optical trapping, radially polarized beam

\section{Introduction}

For manufacturing fine devices and next-generation functional parts, there is a demand for fabrication technology of three-dimensional microstructure. For example, in order to create metamaterial expected to be applied to optical camouflage and super lens, it is necessary to construct an artificial dielectric with a metal structure of several tens of nanometers. So far several microstructural fabrication methods using focused beams such as two-photon reduction method [1] and focused ion beam [2] have been studied. However, it is difficult to process threedimensional microstructures in range smaller than $100 \mathrm{~nm}$ by conventional methods. In addition, majority of conventional micro fabrication techniques using focused beam has to be conducted in liquid phase, which leads to a problem that the structures can be destroyed of deformed by

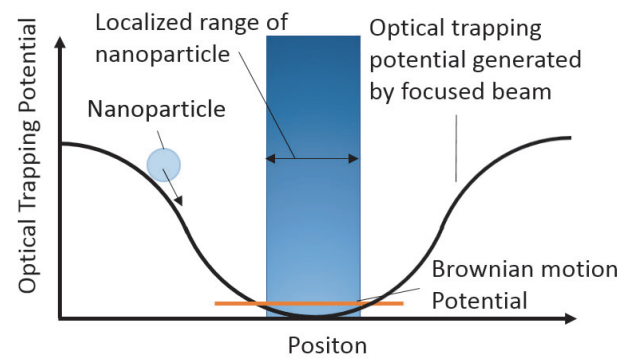

Figure.1 Confinement based on optical trapping potential *yokei@nanolab.t.u-tokyo.ac.jp; phone +81-3-5452-5183 the surface tension of liquid when getting the structures out of liquid.

In this research, we propose an additive method by localizing nanoparticles based on optical trapping potential in air condition[3]. In this report, we aim to improve the optical trapping efficiency to gather the nanoparticles into focal spot by utilizing the radial polarization. The component in the direction of the optical axis of the electric field near the focal spot becomes remarkably strong[4], which is important in processing speed of the proposed three-dimensional fabrication method .

\section{Concept of laser additive processing based on optical trapping potential}

When nanoparticles are exposed to a focused beam, an optical trapping potential would be evoked. The range of nanoparticles' Brownian motion would be limited, and the position of nanoparticles would be confined into the central area of the optical trapping potential as shown in Fig.1. We propose the following processing method utilizing this phenomenon.

As shown in Fig.2, a volatile solvent airborne droplet containing nanoparticles is supplied near the focused beam, and the airborne droplet is localized at the valley position of the optical trapping potential by the focused beam. During or after the airborne droplet being confined by the optical trapping potential of the focused beam, the volatile solvent would 
vaporize so that only the nanoparticles would be left in the location where the airborne droplet was confined. By relatively three-dimensionally scanning the substrate and the focused beam, nanoparticles are localized at desired positions one after another, and the particles are adhered to each other by surface force such as atomic force, and finally the three-dimensional structure could be additively fabricated.

Conventionally, several energy threshold based processing techniques were proposed, which are difficult to maintain the processing energy. We proposed to use optical trapping potential, on which processing resolution is expected to easy to control although potential of Brownian motion was to be considered. By doing so, it is expected to process microstructures with as narrow as sub $100 \mathrm{~nm}$ structures. Moreover, since this method is not premised on processing in solution and can be processed directly in air condition, we can avoid the problem of destruction of the processed object during solution drying due to the above solution surface tension.

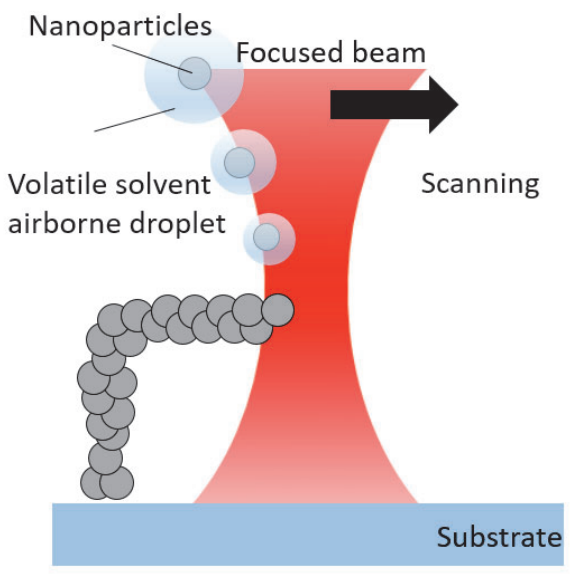

Figure.2 Conceptual diagram of proposed method

\section{Airborne droplet particles trapping}

\section{experiment using radially polarized beam}

In order to realize three-dimensional structuring of fine particles by the proposed method, we conduct basic experiment to verify improvement of optical trapping efficiency using radially polarized beam. In the radially polarized beam, the polarization direction is radially distributed in the cross section of the beam and the component in the direction of the optical axis of the electric field becomes remarkably strong near the focal point of the focused beam. By using this, the improvement of the optical trapping efficiency can be expected.
In this experiment, optical trapping of airborne droplet particles in air condition using a linearly polarized beam and a radially polarized beam was performed. We qualitatively examined the optical trapping efficiency improvement by radially polarized beam.

\section{Experimental Setup}

An experimental setup for a basic experiment using a radially polarized beam was designed and constructed as shown in Fig.3. A fiber laser (continuous wave, linearly polarized) with a wavelength of $1064 \mathrm{~nm}$ and a maximum output of $20 \mathrm{~W}$ is used as the light source. In this experiment, a radially polarized beam is generated by using a radial polarization converter. That is composed of a twisted nematic liquid crystal uniformly wrapped in an annular shape and can convert the linearly polarized beam into a beam that has a continuous radial polarization distribution. Fig. 3 shows the generated radially polarized beam.

For the sample, water airborne droplet in a diameter of about $10 \mu \mathrm{m}$ were used. Also, in order to trap airborne droplet, a chamber was used to accommodate the airborne droplet particles in a fixed place.

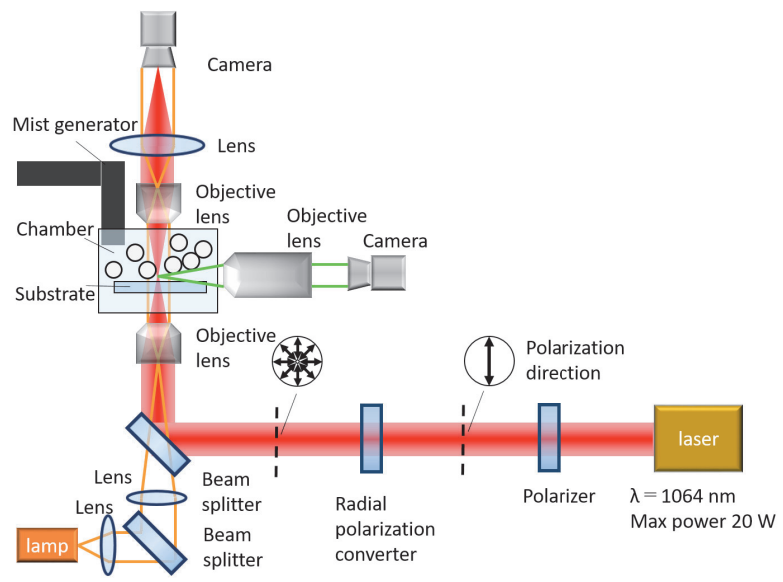

Figure.3 Schematic diagram of the experimental setup

2. Optical trapping of airborne droplet in air condition To test the functionality of the experimental setup, optical trapping airborne droplet in air condition using linearly polarized beam was performed as shown in Fig.4(a). Fig.4(a) shows the trapped airborne droplet observed from the side observation system. The power of the laser near the focal point is about $0.5 \mathrm{~W}$. The light that can be observed is the scattered light of the airborne droplet that is visible when the airborne droplet is irradiated on the laser. When the position of the lower objective lens is moved upward, the scattered light also shifts 
upward as shown in Fig.4(b). When the light source is shut off, the scattered light becomes invisible as shown in Fig.4(c). Therefore, it can be confirmed that the optical trapping was successful. The scattered light in Figure.4(c) can be seen, when airborne droplet is not trapped. This light is considered to be a light scattered at the upper objective lens.

\section{Radially polarized beam}

To trap the airborne droplet using radially polarize beam, radial polarized beam was generated. The Cross sectional intensity distribution of linearly polarized beam coming out of the light source can be seen as shown in Figure.5(a). When a radial polarization converter was incorporated in the experimental setup, the cross sectional intensity distribution of the radially polarized beam can be seen in Figure.5(b) [4].

Since the functionality of the experimental setup was verified and radially polarized beam was generated, comparative verification experiment using linearly polarized beam and radially polarized beam will be performed.

\section{Conclusion}

In this research, we proposed a three-dimensional microstructure processing method by localization of fine particles based on optical trapping potential. In this report, in order to realize three-dimensional structuring of fine particles by the proposed method, fundamental optical trapping of the airborne droplet experiment was performed and radially polarized beam was generated. In the future, comparative verification experiment using linearly polarized beam and radially polarized beam will be performed.

\section{Acknowledgements}

This study was supported by JSPS Grant-in-Aid for Scientific Research (KAKENHI) (Grant No. 17H04900) and the Japan Prize Foundation.
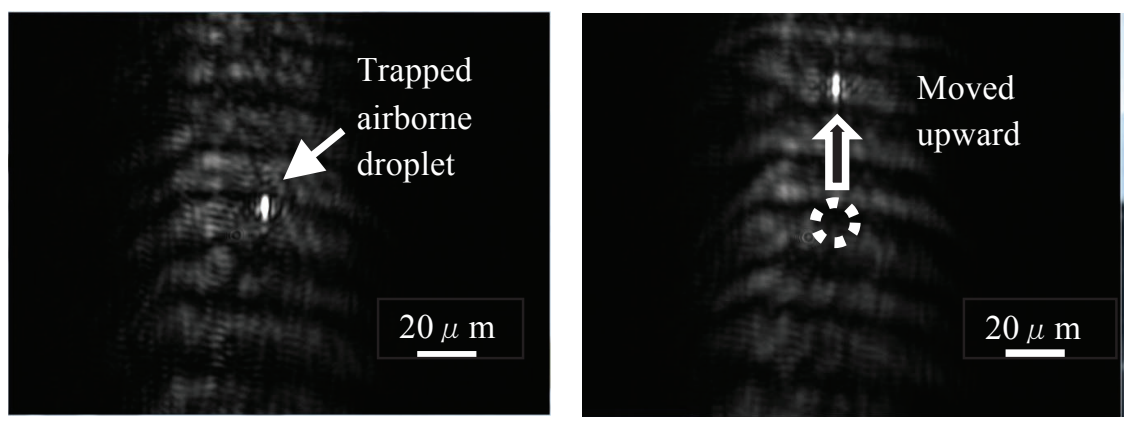

(a)trapped

\section{References}

[1]T.Tanaka, A.ishikawa and S. kawata .,"Two-photon-induced reduction of metal ions for fabricating three-dimensional electrically conductive metallic microstructure," Appl. Phys. Lett. 88,081107 (2006).

[2]S.Matsui, T.Ka ito, J.Fujita, M. Komuro, K.Kanada and Y.Haruyama., "Three-dimensional nanostructure fabrication by focused-ion-beam chemical vapor depositon,” J. Vac. Sci. Technol. B., 18,6,3181(2000).

[3]M.Hayashi, M.Michihata, K.Takamasu and S.Takahashi., "Basic study on laser additive processing for nanostructures based on optical trapping potential," Proc. International Conference of Precision Engineering (ICPE), B-6-1.(2018)

[4]M.Michihata, T.Hayashi and Y.Takaya., "Measurement of vertical and lateral trapping stiffness of optical tweezers in air using radially polarized beam," Applied Optics, pp.61436151.(2009)
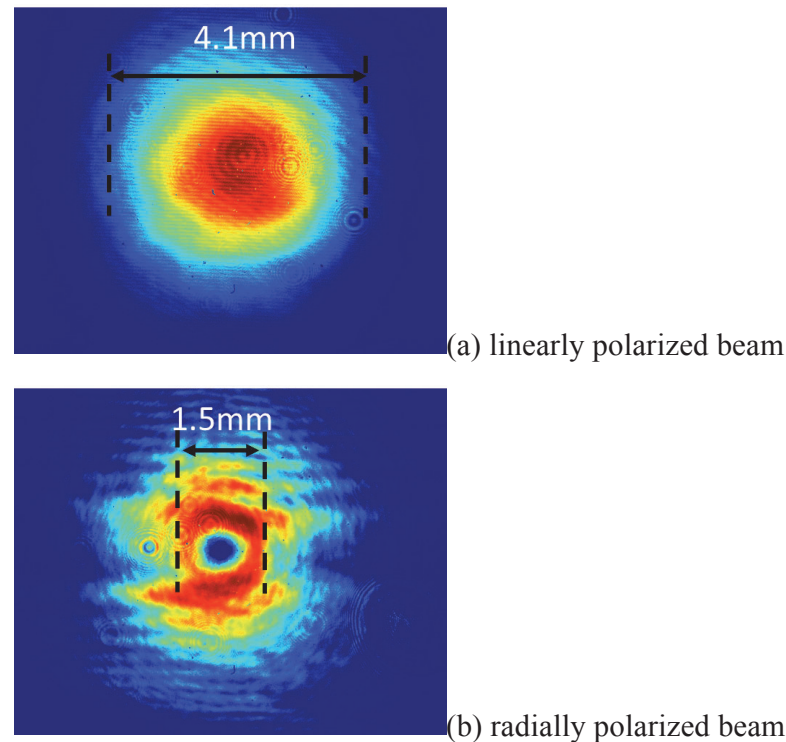

(b) radially polarized beam

Figure. 5 Cross sectional intensity distribution of beam

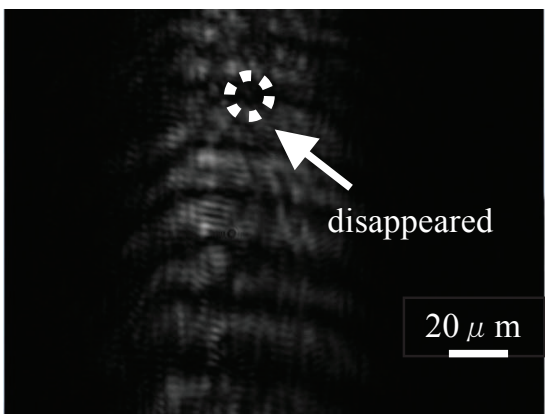

(c) disappeared

Figure.4 Trapped airborne droplet in air condition 


\title{
Evaluation of influences of thin lubricant on fringe projection
}

\section{measurements}

\author{
Sebastian Metzner*a, Tamara Reuter ${ }^{\mathrm{a}}$, Tino Hausotte ${ }^{\mathrm{a}}$ \\ âFriedrich-Alexander-Universität Erlangen-Nürnberg (FAU), Nägelsbachstr. 25, 91052 Erlangen, \\ Germany
}

\begin{abstract}
Sheet bulk metal forming processes pose challenges for fringe projection systems, as the lubricant applied for forming could influence the measurement result. The lubricant layer has an expected film thicknesses smaller than $35 \mu \mathrm{m}$ after the forming process. This paper presents an approach for the measurement of the lubricant layer influence on fringe projection measurements. The lubricant (Beruforge 152DL) used for the forming process and investigated in this paper has special characteristics like a water-based basic fluidity and wax particles for friction reduction as component of the liquid. A newly developed fluid film thickness standard represents the film thickness in a continuous range. This paper shows the impact on the standard deviation of the measurement results and a significant deviation of the detected surface on fluid film thickness standard with the applied lubricating film.
\end{abstract}

Keywords: fringe projection system, lubricant measurement, sheet bulk metal forming

\section{Introduction}

Sheet bulk metal forming as a new manufacturing process challenges optical measurement technologies. The increase in the number of small functional elements and the surface characteristics make a measurement more complex and effortful. The formed surfaces combine diffuse and direct reflecting areas. Especially optical measuring systems reach their limit with these varying surface properties [1]. In addition, lubricants like Beruforge 152DL (BF152DL) are applied on the workpiece before the forming process. After the forming process, the expected lubricant layer thickness lies between $5 \mu \mathrm{m}$ and $35 \mu \mathrm{m}$. The properties of the lubricant are a water-based basic fluidity, wax particles and a milky consistency, which makes measurement with optical measuring instruments more difficult. The paper presents a solution to measure the lubricant within the expected film thickness to evaluate the impact on the measurement result of the fringe projection system.

\section{Related Work}

Various paper already dealt with thin film measurements with fringe projection systems. Palousek et al. [2] investigated the influence of matt coatings, titanium coating or chalk powder spray. They determined the measurement deviation and the variance. Mendericky et al. [3] performed an acceptance test to evaluate the eligibility and accuracy of the fringe projection system. They therefore measured the thickness of antireflection coatings and coatings to matt the surface. Now the most common methods to measure film thicknesses are nondestructive optical methods like ellipsometric, reflectometric and interferometric measurements [4]. However, ellipsometry and reflectometry are only suitable for the measurement of small surfaces, as they only measure a single point at a time. A large surface leads to an increasing measurement time [5]. Interferometric 3Dprofile measurements of thin layers have their preliminary application in the micro electronic industry $[5,6]$. To measure film thickness on sheet bulk metal formed parts it is suitable to use the fringe projection method, as this is a fast and extensive method to measure these geometries.

\section{Experimental setup}

This chapter presents the developed fluid film thickness standard for the optical measurement of thickness dependent thin film properties, describes the results of the focus variation measurements and finally presents the measurement setup for the fringe projection measurements.

\section{Developed fluid film thickness standard}

The fluid film thickness standard (Figure 1) was realized as a $30 \mathrm{~mm} \times 30 \mathrm{~mm}$ square metal block with a height of $10 \mathrm{~mm}$. To measure the film thicknesses a wedge - shape

*sebastian.metzner@fmt.fau.de; phone +49 (0)9131 8520442 
was milled in the middle of the standard (green). The wedge cavity covers a thickness range from $6 \mu \mathrm{m}$ to $100 \mu \mathrm{m}$. The absolute heights can be determined with two planes (black) that are positioned next to the wedge and use as common reference plane. These areas have higher requirements to the surface roughness and parallelism. The principle for the measurement of thicknesses is known from DIN 32567-4:2015-06-00. Two plateaus are placed at the thin and thick end of the wedge cavity for the in- and outflow of a lubricant. The plateaus have a height of $60 \mu \mathrm{m}$ and $5 \mathrm{~mm}$. The first plateau includes a hole for the inflow, in which a medical tube with a closure is inserted, so that the lubricant can be poured in. A small drain on the thin plateau allows the extra lubricant and air to escape. Various engraved reference lines (red) ensure the repeatability of the measurements and data evaluation regarding the lateral position and orientation. An attached glass plate ensures that the lubricant does not form any bulges and thus lies within the defined thickness range. The glass is hold in place with a specially designed cover plate. This plate allows the optical measurement of the thin layers and prevents the leakage of the lubricant film from the wedge. Figure 1 shows the fluid film thickness standard with and without the cover plate.
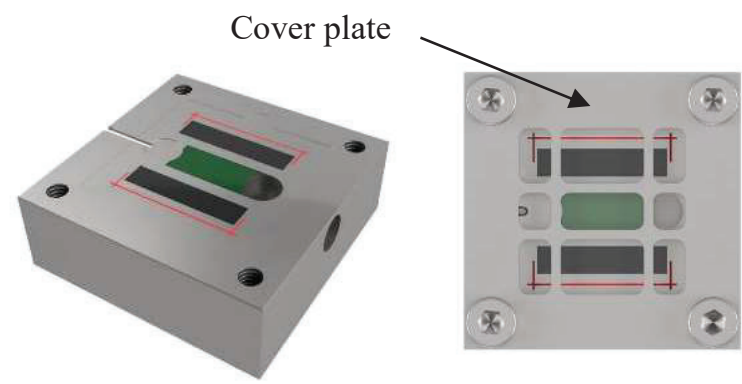

Figure 1. CAD model of the fluid film thickness standard with and without the cover plate

The lubricant is inserted into the fluid film thickness standard with a syringe and flows through the $5 \mathrm{~mm}$ plateau into the wedge cavity. After the tube is closed, the liquid remains in the fluid film thickness standard as illustrated in Figure 2. It shows the fluid film thickness standard filled with the lubricant investigated in this paper BF152DL, the cover plate and the tube. In the case of optical measurements, the experimental setup only provides information about the effects of the lubricant on the fringe projection measurements and not about the shape of the surface. The fringes are reflected within the semitransparent lubricant.

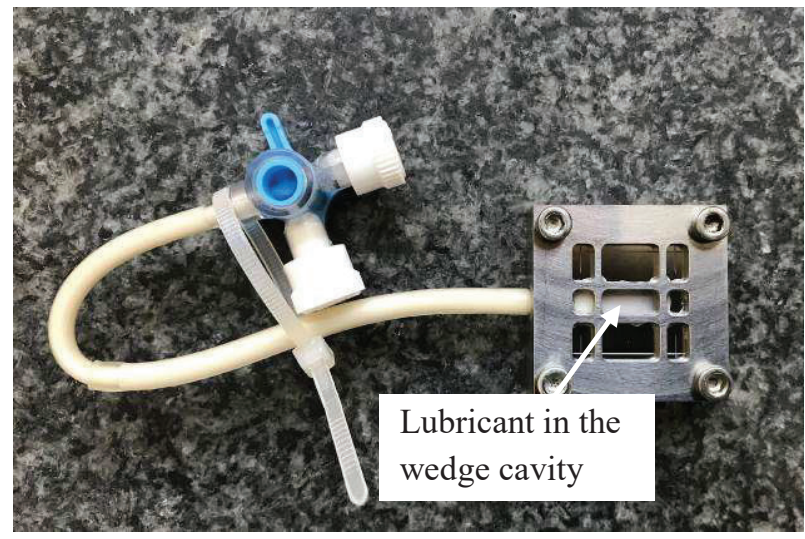

Figure 2. Fluid film thickness standard with cover plate filled with the lubricant BF152DL

\section{Reference measurement of the empty fluid film thickness standard using focus variation method}

In order to validate the wedge cavity geometry and roughness, the fluid film thickness standard was first measured with the focus variation measurement system Alicona InfiniteFocus G4. The measurement was performed using the $5 x$ objective, which leads to a axial resolution specified by Alicona of approximately $332 \mathrm{~nm}$ and a lateral resolution of approximately $4 \mu \mathrm{m}$. The measuring range covers the area of the engraved reference lines as shown in Figure 3.

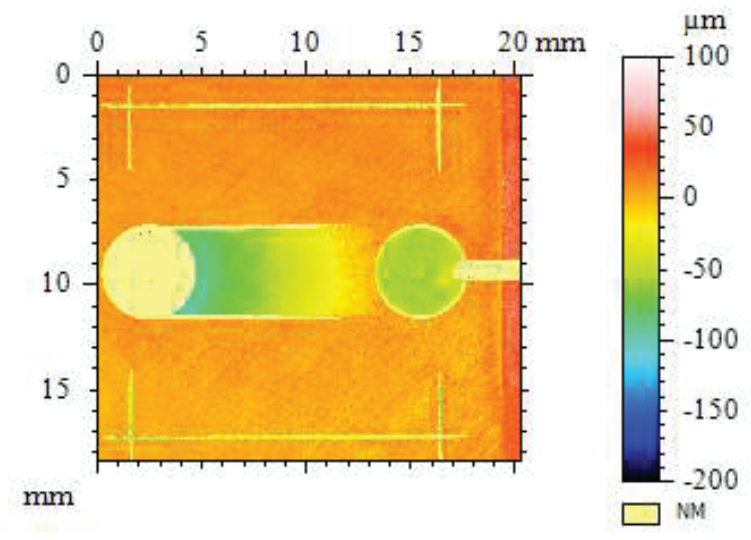

Figure 3. Dataset of the focus variation measurement showing the geometry of the fluid film thickness standard with engraved reference lines 
The roughness $R_{\mathrm{a}}$ on the wedge plane is $5.5 \mu \mathrm{m}$ (cut-off wavelength $0.8 \mathrm{~mm}$ ). Although the very smooth surface allows very accurate measurements between the reference planes and the wedge plane, the low roughness makes optical measurements more difficult. The roughness is thus a tradeoff between a high absolute accuracy and a low roughness for better detection of the surface using optical measurement systems.

\section{Measurement setup with fringe projection sensors}

The measurement setup is part of an existing multisensor setup at the Institute of Manufacturing Metrology that consists of various fringe projection sensors and a high-precision hexapod for positioning [7]. For the measurement of the standard deviation, the fringe projection system MicroCad pico 1.0 of the company LMI Technologies Inc. (formerly GFMesstechnik $\mathrm{GmbH}$ ) was used. The measurement range of the sensor is $12.4 \mathrm{~mm}$ x $7.9 \mathrm{~mm} \times 4.4 \mathrm{~mm}$. The specified resolution is $17 \mu \mathrm{m}$ in lateral direction and $1 \mu \mathrm{m}$ in vertical direction. Figure 4 shows the multi-sensor measurement setup and the fluid film thickness standard placed on the hexapod of the fringe measurement system.

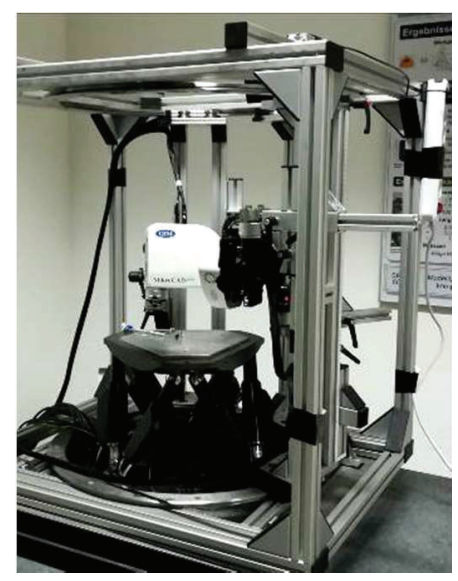

a)

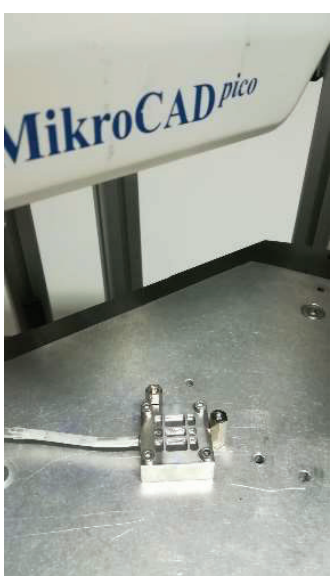

b)
Figure 4. Multi-sensor measurement setup a) and the fluid film thickness standard placed on the hexapod b).

\section{Results and Discussion}

\section{Standard deviation of repeated measurements}

Repeated measurements $(n=25)$ are used to show the effect on the fringe projection system with different surface properties (measurements of the fluid film thickness standard itself and the thin lubricant). The distances of each point of the measurement to the reference planes was calculated. A direct comparison of the point cloud is not possible due to a slightly changed position of the calculated points. Therefore, the nearest neighbors of the points are calculated and the distances of the related points to the common reference plane were calculated.

Figure 5 shows that the standard deviation without BF152DL in the wedge cavity is much higher than the standard deviations with the lubricant in the small thickness areas. This is due to a very directionally reflecting surface of the wedge. However, the standard deviations are homogeneously distributed over the entire area. The measurements with the BF152DL show an increasing standard deviation with increasing lubricant thickness. It is possible that with a higher lubricant thickness, the fringe pattern is no longer reflected on the surface. This hypothesis would mean that due to the initiated wax particles in the lubricant, a random reflection of the fringes lead to larger standard deviations in the measurement results.

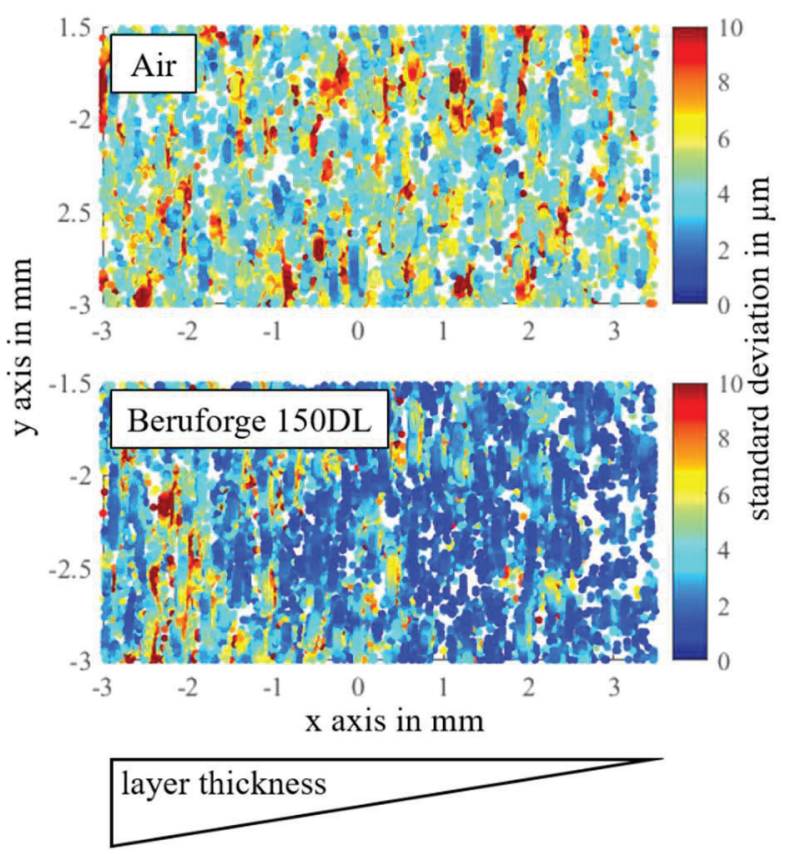

Figure 5. Standard deviation in $\mathrm{mm}$ of the measurement with BF152DL and with air.

\section{Comparison of absolute heights in profile}

This chapter describes the absolute height differences from the reference plane to the measurement results on the 
wedge plane. For the measurement of the heights, the fringe projection system MicroCad pico 0.3 was used. The specified vertical resolution is $2.5 \mu \mathrm{m}$ in lateral and $0.3 \mu \mathrm{m}$ in vertical direction. Figure 6 shows the absolute deviation of the measured distance to the reference plane. The figure shows the wedge in side view similar to the side view of Figure 5.

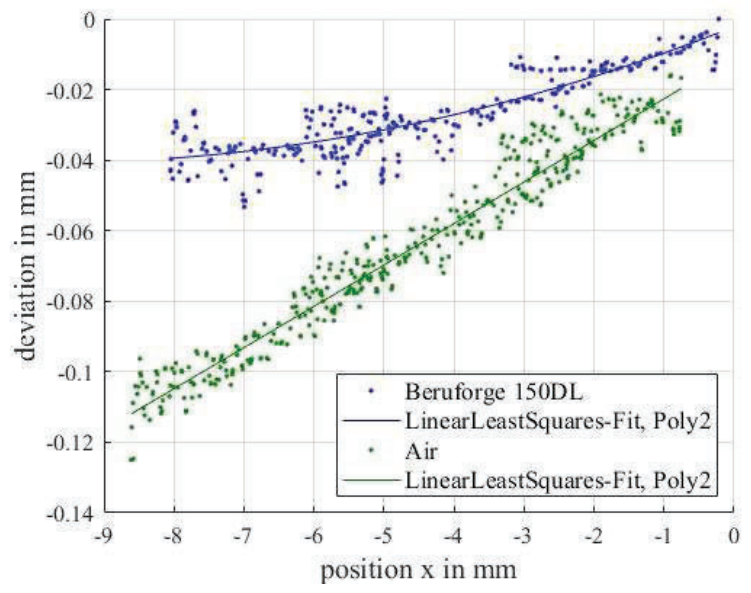

Figure 6. Cross-section of the wedge cavity with the deviation from the reference plane.

The measurement without BF152DL (green) shows a well-detected wedge with a linear decrease of the height. The figure includes the detected geometry points of the measurement as well as a polynomial fit of order $k=2$. The measurement with BF152DL shows that the lubricant is detected reliably in certain heights so that the deviation from the reference plane is significantly lower in these areas. With increasing layer thickness, the deviations increase slightly. Ideal reflection on the lubricant surface would have resulted in a horizontal line across all layer thicknesses. These results are consistent with the standard deviation shown in section 4.1. As the fringe of the measurement system do not capture the surface of the lubricant, a larger deviation is detected. The result converges to a deviation of about $40 \mu \mathrm{m}$ for a larger layer thickness. Within the expected lubricant heights during and after sheet bulk metal forming a nonlinear deviation is recognized.

\section{Summary}

The measurements show that the detection of BF152DL is possible and leads to a significant deviation in the measurement result with the fringe projection measurement systems used. The high standard deviations are even reduced at low layer thicknesses of the lubricant. However, due to an uneven distribution of the lubricant film on the workpiece after a standard measurement, it is difficult to calculate systematic deviation in the measurement and to include them it in the measurement result.

\section{Acknowledgement}

The work is funded by the German Research Foundation (DFG) as part of the Collaborative Research Centre SFB / TR73 in the sub-project A6 and was carried out at the Friedrich-Alexander-Universität Erlangen-Nürnberg (FAU).

\section{References}

[1] Metzner, S., Ramesh, R., Müller, A., Hausotte, T. "Evaluation of reflection properties of sheet bulk metal formed parts by pixel wise analysis of camera images provided for triangulation measurement", ITGFachbericht 281 Sensoren und Messsysteme , 203-206 (2018).

[2] Palousek, D., Omasta, M., Koutny, D., Bednar, J., Koutecky, T., Dokoupil, F., "Effect of matte coating on 3D optical measurement accuracy", Optical Materials 40, 1-9 (2015).

[3] Mendricky, R., "Determination of measurement accuracy of optical 3D scanners", MM Science Journal, 1565-1572 (2016).

[4] Hlubina, P., Luňáček, J., Ciprian, D., Chlebus, R., "Spectral interferometry and reflectometry used to measure thin films", Applied Physics B 92(2), 203-207 (2008).

[5] Ghim, Y. S., Kim, S. W., "Fast, precise, tomographic measurements of thin films", Applied Physics Letters 91(9), 091903 (2007).

[6] Ghim, Y. S., Kim, S. W., "Dispersive white-light interferometry for thin-film thickness profile measurement", Proc. SPIE 5856, 419-426 (2005).

[7] Metzner, S., Hausotte, T., Loderer, A. "Ganzheitliche dimensionelle Messung von blechmassivumgeformten Bauteilen", tm-Technisches Messen 84, 600-609 (2017). 


\title{
2D and 3D Vision Based Face Recognition System
}

\author{
Mengyue Zhang*a, Bin Lin ${ }^{\mathrm{a}}$ \\ ${ }^{a}$ Zhejiang University, Hangzhou, China
}

\begin{abstract}
Traditional 2D face recognition algorithm is mature but easily affected by external environment such as makeup and light. Face recognition based on 3D methods contains more information compared to 2D, and is stable under different external conditions. This paper put a lot of face data into VGG deep learning convolution neural network, and then establish 2D and 3D face databases respectively. Using Kinect v2 to obtain 2D color images and 3D point cloud information. After preprocessing of 2D images and 3D point cloud respectively, calculate the Euclidean distance by convolution neural network; Collected by weighted square sum algorithm of point cloud and those from 3D face database and calculate 3D similarity. Finally, through the weighted combination of two kinds of similarity, get the final recognition result. Combining the $2 \mathrm{D}$ and $3 \mathrm{D}$ recognition advantages, the accuracy and robustness of the system are strengthened.
\end{abstract}

Keywords: face recognition, 2D/3D fusion vision, convolutional neural network, kinect.

\section{Introduction}

As one of the most effective ways of personal identity verification in modern society, face recognition has important application value. Traditional 2D face recognition technology is common and mature, which can be divided into geometric features, neural network-based recognition methods, elastic model-based recognition methods and so on. Among them, face recognition method based on deep learning convolutional neural network has been greatly developed in recent years, and some deep learning network models based on a large number of face data are constructed.

3D face recognition has received wide attention due to its superiority to $2 \mathrm{D}$ recognition. The difference of $3 \mathrm{D}$ face recognition and $2 \mathrm{D}$ face recognition is that the data source is different. Firstly, 3D data does not change with environmental conditions such as lighting conditions, viewing angles, or makeup. Secondly, 3D data has spatial shape features, richer information than 2D image. 3D face recognition technique has become one of the research hotspots in the field of face recognition.

In this paper, we propose a $2 \mathrm{D} / 3 \mathrm{D}$ vision based face recognition system, which combines the advantages of 2D and 3D face recognition, can recognize human faces fast and accurately against the influence from viewing angle, environment light and makeup.

\section{2D recognition}

\section{Experiment}

We preprocess 494,414 image data of 10,575 people in the CASIA-webface face database, and divide them into training set and test set to train and optimize VGG network.

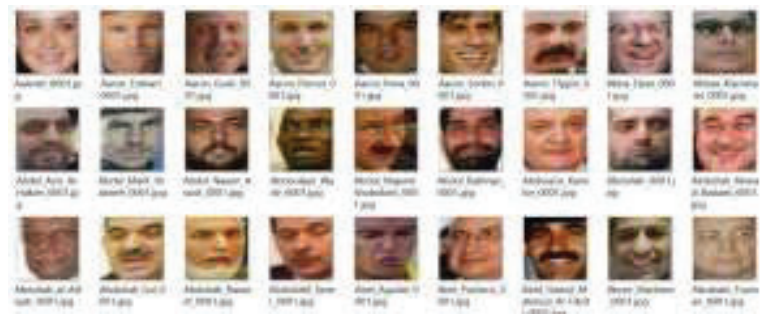

Figure 1. Preprocessed face images input into neural network training

We use deep learning framework Caffe in windows10 machine with Python language, setting 16-layer network structure parameters of VGG convolutional neural network, and generates configuration file prototxt. It is trained to minimize the logistic regression loss function , and the parameters of the connection layer is optimized. Then the final caffe model of VGG is obtained. 


\begin{tabular}{|c|c|c|c|c|c|}
\hline \multicolumn{6}{|c|}{ ConvNes Conf guration } \\
\hline$A$ & ALLRN & B & $\mathrm{C}$ & D & $\mathrm{E}$ \\
\hline $\begin{array}{c}11 \text { weight } \\
\text { layers }\end{array}$ & $\begin{array}{c}\text { It weight } \\
\text { layers }\end{array}$ & $\begin{array}{l}13 \text { weight } \\
\text { layers }\end{array}$ & $\begin{array}{l}\text { 16. weighi } \\
\text { layers }\end{array}$ & $\begin{array}{l}16 \text { weigh } \\
\text { layers }\end{array}$ & $\begin{array}{l}19 \text { weighi } \\
\text { lagen }\end{array}$ \\
\hline \multicolumn{6}{|c|}{ Beput $(224 \times 224$ RGB image) } \\
\hline $\operatorname{cow} 3-64$ & $\begin{array}{l}\text { comv3.64 } \\
\text { I.RV }\end{array}$ & $\begin{array}{l}c 0003.64 \\
\text { conv } 3-64\end{array}$ & $\begin{array}{l}\operatorname{com} 3-64 \\
\operatorname{com} 3-64\end{array}$ & $\begin{array}{l}\cos 3.64 \\
\cos 3-64\end{array}$ & $\begin{array}{l}\cos 33.64 \\
\cos 3-64\end{array}$ \\
\hline \multicolumn{6}{|c|}{ maxpoed } \\
\hline $\cos 3-128$ & comv3-128 & $\begin{array}{l}\cos 3-128 \\
\operatorname{con} 3-128\end{array}$ & $\begin{array}{l}\operatorname{cosen} 3-12 \pi \\
\cos 3-128\end{array}$ & $\begin{array}{l}\cos 33-128 \\
\cos 3-128\end{array}$ & $\begin{array}{l}\cos 3-128 \\
\cos 3-128\end{array}$ \\
\hline \multicolumn{6}{|c|}{ maxpood } \\
\hline $\begin{array}{l}\text { conv } 3=256 \\
\text { conv } 3.256\end{array}$ & $\begin{array}{l}\text { conv } 3.256 \\
\text { canv3.256 }\end{array}$ & $\begin{array}{l}\cos 3.256 \\
\cos 3.256\end{array}$ & $\begin{array}{l}\operatorname{com} 3.256 \\
\cos 3.256 \\
\text { coav1-2.56 }\end{array}$ & $\begin{array}{l}\operatorname{com} 3.256 \\
\text { comw } 3.256 \\
\text { coens.3-256 }\end{array}$ & $\begin{array}{l}\cos 3.256 \\
\cos 3.256 \\
\cos 3-256 \\
\cos 3-256\end{array}$ \\
\hline \multicolumn{6}{|c|}{ maxpool } \\
\hline $\begin{array}{l}\text { convy-512 } \\
\text { conv } 3-512\end{array}$ & $\begin{array}{l}\text { colve } 35512 \\
\text { conv } 3512\end{array}$ & $\begin{array}{l}\mathrm{cow} / 3-512 \\
\cos 3-512\end{array}$ & $\begin{array}{l}\cos 3.512 \\
\cos 3.512 \\
\operatorname{conv1.512}\end{array}$ & $\begin{array}{l}\text { cores } 3-312 \\
\text { com } 3.512 \\
\text { conv } 3-512\end{array}$ & $\begin{array}{l}\cos 3-512 \\
\cos 3-512 \\
\cos 3.512 \\
\cos 3-512\end{array}$ \\
\hline \multicolumn{6}{|c|}{ maxpood } \\
\hline $\begin{array}{l}\text { coniv3-312 } \\
\text { cow } 3-512\end{array}$ & $\begin{array}{l}\text { comv3-512 } \\
\text { cunv3-512 }\end{array}$ & $\begin{array}{l}\cos 3-312 \\
\sin 3,512\end{array}$ & $\begin{array}{l}\operatorname{coser} 3.312 \\
\operatorname{com} 3.512 \\
\operatorname{conv1.512}\end{array}$ & 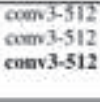 & $\begin{array}{l}\operatorname{cosw} 3-512 \\
\cos 3-512 \\
\cos 3-512 \\
\cos 3.512\end{array}$ \\
\hline \multicolumn{6}{|c|}{ 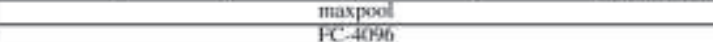 } \\
\hline & & FC & 496 & & \\
\hline \multicolumn{6}{|c|}{ FC-40\%6 } \\
\hline \multicolumn{6}{|c|}{$F(-100)$} \\
\hline
\end{tabular}

Figure 2. VGG network architecture and parameters

Based on the trained VGG model, we put the user's face image which is to be identified into the network, make feature extraction and calculation, and compare it with the face database by calculating the average Euclidean distance between the image to be tested and image in the database.

\section{3D recognition}

3D face recognition includes the following steps: First, obtain point cloud of face through 3D-data acquisition device(Kinect v2). Next, make pretreatment of edge detection, division and noise removal. Then find the essential attributes between the features, and make 3D reconstruction. Finally, classify the extracted 3D face features and output the results.

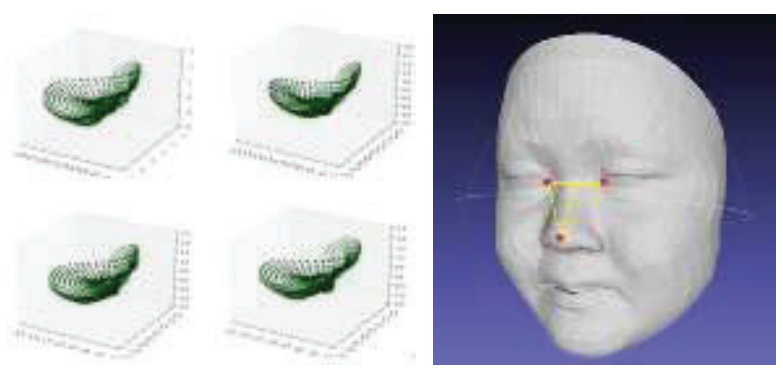

Figure 3. Point clouds and reconstructed 3D face model

\section{2D/3D fusion}

Based on environmental condition, give appropriate weight distribution to $2 \mathrm{D}$ and $3 \mathrm{D}$ result, carry out the fusion similarity in decision-making level.

The principle of weight allocation is to optimize the recognition accuracy after decision fusion. Therefore, for different experimental environments, we need to allocate weights according to specific circumstances. In the environment of poor illumination condition, the result of two-dimensional image recognition has lower credibility, but the result of three-dimensional image recognition has higher credibility, so it is necessary to assign higher weight to the result of three-dimensional recognition; in the environment of better illumination condition, the result of two-dimensional image recognition has higher credibility, so it can assign smaller weight to the result of three-dimensional recognition. Heavy.

\section{Results and Discussion}

We test the system in 10 users database, each person with 10 front color 2D images and 3 sets of 3D point cloud data. The users collects a 2D color image and a set of 3D point cloud data through Kinect. The similarity calculation is performed with ten users in the test library, and the highest similarity is taken as the final recognition result. The accuracy of three recognition methods of twodimensional, three-dimensional and two-dimensional three-dimensional fusion is calculated separately.

The accuracy of the system is tested after a certain number of samples as shown in the following table.

Table 1. Test results in different lighting condition

\begin{tabular}{|c|c|c|c|}
\hline \multirow{2}{*}{$\begin{array}{c}\text { Experimental } \\
\text { condition }\end{array}$} & \multicolumn{3}{|c|}{ Recognition Accuracy } \\
\cline { 2 - 4 } & $2 \mathrm{D}$ & $3 \mathrm{D}$ & $\begin{array}{c}2 \mathrm{D} / 3 \mathrm{D} \\
\text { fusion }\end{array}$ \\
\hline $\begin{array}{c}\text { Good } \\
\text { illumination }\end{array}$ & $91 \%$ & $86 \%$ & $89 \%$ \\
\hline $\begin{array}{c}\text { Common } \\
\text { illumination }\end{array}$ & $78 \%$ & $84 \%$ & $82 \%$ \\
\hline $\begin{array}{c}\text { Poor } \\
\text { illumination }\end{array}$ & $65 \%$ & $79 \%$ & $71 \%$ \\
\hline
\end{tabular}


This system use a large amount of data to train the image recognition classifier VGG convolutional neural network, and use the trained network model to classify and recognize two-dimensional color face images, combined with deep learning and artificial intelligence to efficiently and accurately measure two-dimensional faces. The idea of fusion in the decision-making layer is proposed. The two-dimensional face recognition and three-dimensional face recognition are complemented and organically combined, and the face information is fully utilized.

Due to the limitations of experimental conditions and data volume, the research in this paper is not complete enough. In order to further explore this topic, subsequent research can be carried out mainly in the following aspects:

(1) Expansion of the size of the face database:

Due to the specific conditions required for 3D model acquisition, the size of the face database established in this paper is small. Therefore, when testing the performance of the entire system, the accuracy of debugging is not high enough due to the limited sample capacity.

(2)Research on 3D point cloud matching algorithm:

The 3D point cloud matching algorithm proposed in this paper has specificity and limitations, and the acquired 3D data format is also unique. If the device that collects the 3D data changes, a new algorithm is needed for matching, and the subsequent research can be carried out in the search for the ubiquitous algorithm.

\section{References}

Jia Y, Shelhamer E, Donahue J, et al. Caffe: Convolutional Architecture for Fast Feature Embedding[J]. 2014.

Simonyan K , Zisserman A . Very Deep Convolutional Networks for Large-Scale Image Recognition[J]. Computer Science, 2014.

Paysan P, Knothe R, Amberg B, et al. A 3D Face Model for Pose and Illumination Invariant Face Recognition[C]// IEEE International Conference on Advanced Video \& Signal Based Surveillance. 2009.

Xiang X, Le H A, Dou P, et al. Evaluation of a 3D-aided pose invariant $2 \mathrm{D}$ face recognition system[C]// IEEE
International Joint Conference on Biometrics. 2018. scenes. Transactions of the ASAE 32(2):757-763.

Yi S, Wang $X$, Tang $X$. Deep Learning Face Representation from Predicting 10,000 Classes[C]// IEEE Conference on Computer Vision \& Pattern Recognition. 2014.

Zquez M A, Cuevas F J. A 3D Facial Recognition System Using Structured Light Projection[C]// International Conference on Hybrid Artificial Intelligence Systems. Springer-Verlag New York, Inc. 2014. 


\title{
A multi-axis space coordinate system calibration method for composite line laser measuring systems using non-feature planes and multi-angle spheres
}

\author{
Changda $\mathrm{Xu}^{\mathrm{a}}$, Xiang Zhou *b \\ ${ }^{a}$ School of mechanical engineering, Xi'an Jiaotong University, No.28, Xianning West Road, Xi'an, \\ Shaanxi, 710049, P.R. China \\ ${ }^{\mathrm{b}}$ School of mechanical engineering, Xi'an Jiaotong University, No.28, Xianning West Road, Xi'an, \\ Shaanxi, 710049, P.R. China
}

\begin{abstract}
For Line-Laser sensor products that CCD images are unknown, we present a method for the calibration of Line-Laser sensor measurement system using multi-directional and non-featured planes, and a method for system calibration optimization using multi-angle standard spheres. Through experiments, the accuracy of the line laser measurement system can reach $0.02 \mathrm{~mm}$ for a standard ball with a radius of $12.696 \mathrm{~mm}$.
\end{abstract}

Keywords: Line laser sensor, CMM, Non-contact measurement, calibration, Nonlinear optimization, Non-feature plane

\section{Introduction}

Below is our hardware structure diagram.

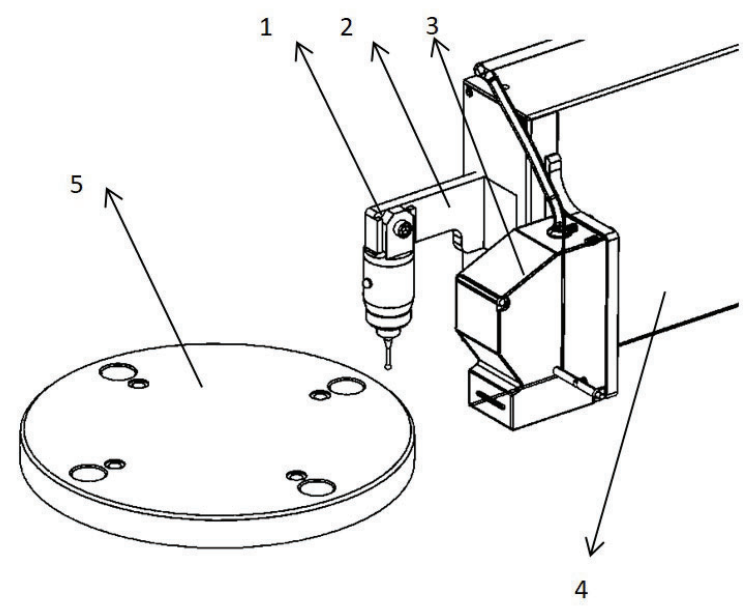

Figure 1. 1- Contact probe, 2- Rigid connector structure, 3- KEYENCE 7020 Line laser sensor, 4- Y-axis of CMM， 5- Turntable

Coordinate measuring machine with Line-Laser sensor for non-contact object measurement of objects is becoming more and more popular. Calibration methods for Line-Laser sensors and measurement systems are generally limited to obtain sensor CCD images. For LineLaser sensor products that have been commercialized, its
CCD images are mostly unknown and cannot be calibrated in a coordinate measuring machine by conventional methods. In this paper, we present a method for the calibration of Line-Laser sensor measurement system using multi-directional and non-featured planes, and a method for system calibration optimization using multi-angle standard spheres.

For a line laser sensor that can acquire a CCD image, the conversion mechanism of the sensor to the measuring machine is usually calibrated by photographing the calibration plate by using the principle of the calibration camera. When the CCD image cannot be obtained, the above method is no longer applicable.

\section{Our Calibration Method}

\section{Preliminary calibration}

As shown in Fig. 2, the measurement points are directly collected on the standard plane by the contact probe, and the plane equation is fitted by the least squares method. The plane equation is as follows.

$$
A x+B y+C z+D=0
$$

We use a line laser sensor to scan the same plane to obtain a series of line laser sensor acquisition points $S_{n}$ and corresponding grating coordinate values $\Delta P_{n}$ in the 
CMM. Where $S_{n}$ and $\Delta P_{n}$ is represented by homogeneous coordinates.

$$
\begin{gathered}
S_{n}=\left[\begin{array}{cccc}
l_{1} & l_{2} & & l_{n} \\
w_{1} & w_{2} & \cdots & w_{n} \\
0 & 0 & & 0 \\
1 & 1 & 1 & 1
\end{array}\right], \\
\Delta P_{n}=\left[\begin{array}{cccc}
\Delta x_{1} & \Delta x_{2} & & \Delta x_{n} \\
\Delta y_{1} & \Delta y_{2} & \ldots & \Delta y_{n} \\
\Delta z_{1} & \Delta z_{2} & \Delta z_{n} \\
0 & 0 & & 0
\end{array}\right]
\end{gathered}
$$

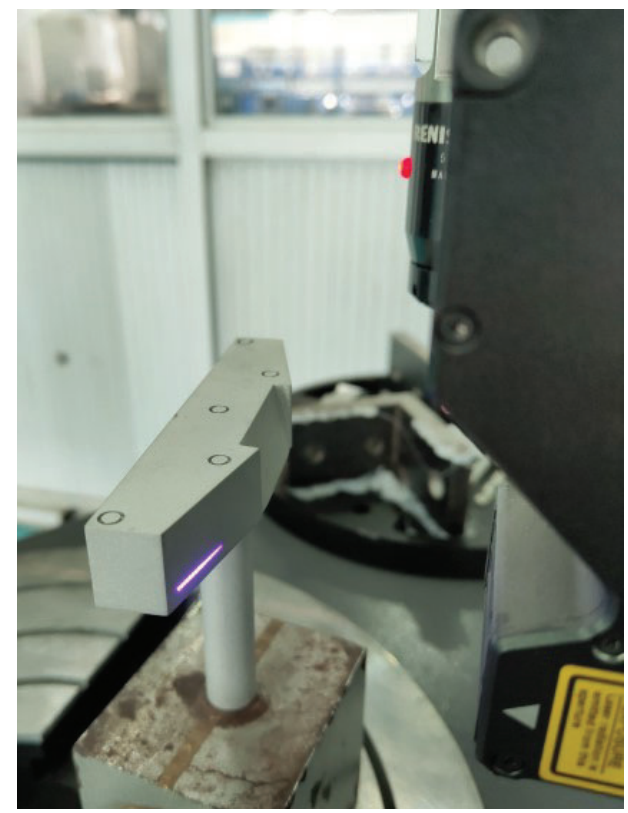

Figure 2. Contact probes and Line Laser sensor are measuring planar calibration blocks

Then we repeatedly scan the other two planes to get the corresponding data, and establish the mathematical model shown in Figure 3.

$O_{M}-X Y Z$ is the machine coordinate system, and the three coordinate axes are the same as the machine grating direction. When the grating is in the zero position, the origin $O_{M}$ is the ruby center position of the contact stylus. At this zero position, the center of the optical axis on the standard working plane of the line laser sensor is $P_{0}\left(x_{0}, y_{0}, z_{0}\right)$ in $O_{M}-X Y Z$.

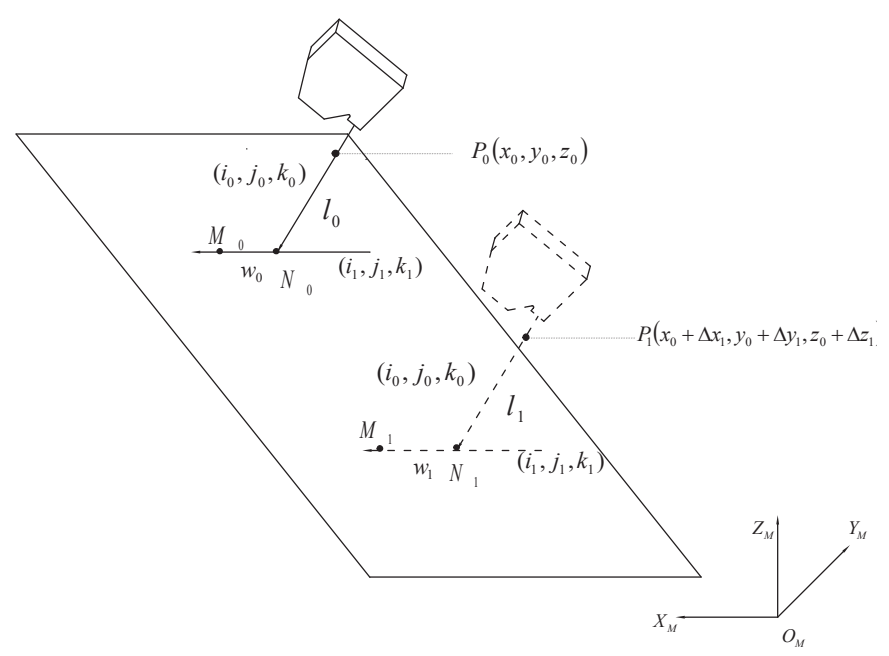

Figure 3. Simplified model for calibration of line laser displacement sensors

When the laser measures a certain standard plane, assuming that $N_{0}$ is the point corresponding to the center of $\mathrm{CCD}$, then the direction of $P_{0} N_{0}$ is the laser exit direction, and this direction is denoted as $\left(i_{0}, j_{0}, k_{0}\right)$, and the laser line length direction vector is $\left(i_{1}, j_{1}, k_{1}\right)$. Suppose a point on the ray is $M_{0}$, the distance from $M_{0}$ to $N_{0}$ is $w_{0}$, and the distance from $M_{0}$ to the standard work plane is $l_{0}$. At this time, the coordinate of $M_{0}$ in $O_{M}-X Y Z$ is $\left(x_{0}, y_{0}, z_{0}\right)+l_{0} \cdot\left(i_{0}, j_{0}, k_{0}\right)+w_{0} \cdot\left(i_{1}, j_{1}, k_{1}\right)$

Mathematical models can be written in matrix form as follows.

$$
\left(K S_{n}+\Delta P_{n}\right)^{T} X=0
$$

In the formula, 


$$
\begin{aligned}
K & =\left[\begin{array}{ll}
R & t \\
0 & 1
\end{array}\right], \quad R=\left[\begin{array}{lll}
i_{0} & i_{1} & i_{2} \\
j_{0} & j_{1} & j_{2} \\
k_{0} & k_{1} & k_{2}
\end{array}\right], \quad t=\left[\begin{array}{l}
x_{0} \\
y_{0} \\
z_{0}
\end{array}\right], \\
S_{n} & =\left[\begin{array}{llll}
l_{1} & l_{2} & & l_{n} \\
w_{1} & w_{2} & \cdots & w_{n} \\
0 & 0 & & 0 \\
1 & 1 & 1 & 1
\end{array}\right], \\
\Delta P_{n} & =\left[\begin{array}{cccc}
\Delta x_{1} & \Delta x_{2} & \Delta x_{n} \\
\Delta y_{1} & \Delta y_{2} & \Delta y_{n} \\
\Delta z_{1} & \Delta z_{2} & \Delta z_{n} \\
0 & 0 & 0
\end{array}\right], \quad X=\left[\begin{array}{l}
A \\
B \\
C \\
D
\end{array}\right] .
\end{aligned}
$$

Then we use the Newton iteration method to solve the equation. We select the objective function as the error distance from the measured point to the fitted plane, and optimize the value of the nonlinear optimization objective function to a minimum. Finally we can get the initial value of the calibration.

\section{Optimization}

Then, we bring the turntable into the overall optimization part of the system. We placed the calibrated standard ball of radius $R_{\text {sphere }}$ on the turntable and calibrated the turntable coordinate system by measuring the standard ball by the contact probe. As shown in Fig. 4, the contact probe and the line laser sensor measure the standard ball at the same angle successively, and obtain the contour data of the standard ball at the same angle. The turntable drives the standard ball to rotate, and then repeats the above measurement steps.

Finally, the equation is solved by the LevenbergMarquardt iterative method. We select the distance from the line laser measurement point to the contact measurement sphere as the objective function, and optimize the objective function to make the distance and minimum.

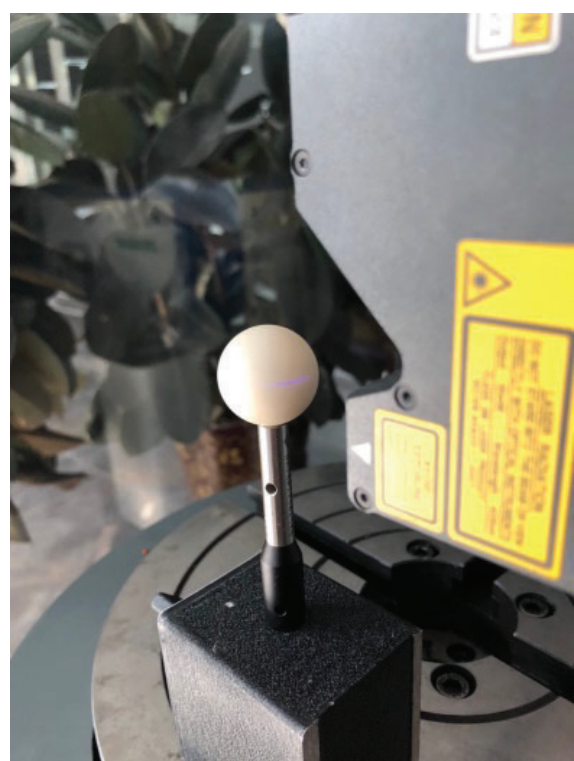

Figure 4. The line laser sensor is measuring the standard ball.

\section{Results and Discussion}

Both simulation analyses and real experiments were conducted. A Line-Laser sensor was used to measure a frosted standard ball with a radius of $12.696 \mathrm{~mm}$. The radius deviation measured by the Line-Laser sensor and the deviation comparing with the center of the ball measured by the CMM were observed. The experimental results show that the radius deviation of the calibration laser sensor measurement system is less than $0.02 \mathrm{~mm}$, and the ball center distance deviation is less than $0.02 \mathrm{~mm}$. Fig 5 shows the measurement results of our standard ball. Compared with the contact probe, the results are shown in Table 1.

This method utilizing non-featured planes simplifies the calibration equipment and can reduce the fitting error when using standard ball from multiple angles for calibration. This method is different from the method of calibrating the single direction of the laser sensor. It can simultaneously calibrate the rotation matrix and translation matrix of the two-dimensional line laser sensor to the coordinate measuring machine, and optimize the global optimal calibration parameters. Finally, we can use this method to get high-precision calibration results. 


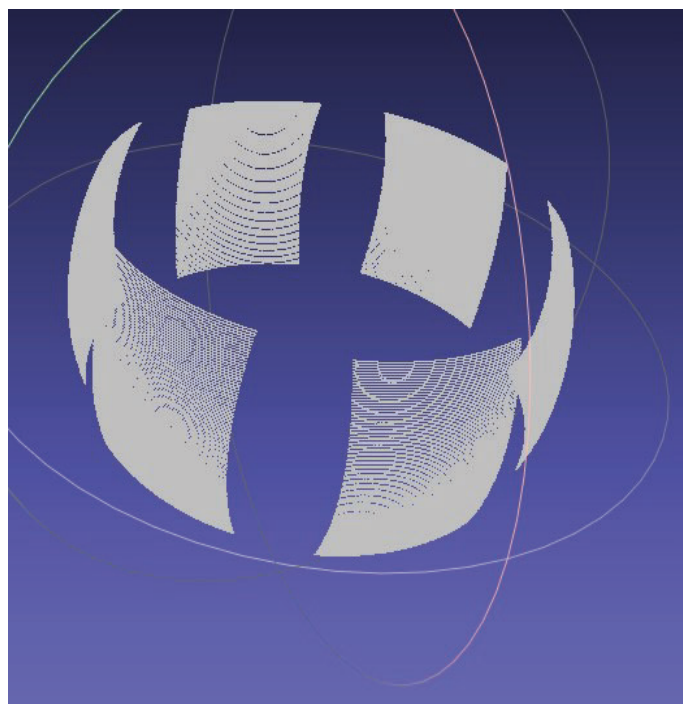

Figure 5. The result of the standard ball measured by a line laser sensor

Table 1. Measuring the center of the sphere and radius with CMM and line laser measurement systems.

\begin{tabular}{cccccc}
\hline Group & 1 & 2 & 3 & 4 & CMM \\
\hline X Coordinate $(\mathrm{mm})$ & 107.9093 & 107.9163 & 107.9188 & 107.9172 & 107.9160 \\
Y Coordinate $(\mathrm{mm})$ & 166.3181 & 166.3280 & 166.3252 & 166.3246 & 166.3252 \\
Z Coordinate $(\mathrm{mm})$ & 48.5299 & 48.5304 & 48.5301 & 48.5292 & 48.5289 \\
Radius $(\mathrm{mm})$ & 12.6855 & 12.6926 & 12.6899 & 12.6889 & 12.696 \\
\hline
\end{tabular}




\title{
Tuning focal length of vari-focal lens for color 3D object reconstruction
}

\author{
Jing-Sheuan Lin, Chun-Jen Weng*, Pi-Ying Cheng \\ Instrument Technology Research Center, National Applied Research Laboratories \\ Department of Mechanical Engineering, National Chiao Tung University \\ Hsinchu, Taiwan \\ cjweng@itrc.narl.org.tw
}

\begin{abstract}
A color 3D object reconstruction system using varifocal lens with various focus algorithms was established in this study. The focal length of the varifocal lens can be regulated by varying the focal power. In this study, four categories of algorithms are applied into the system. The focal length of the system is adjusted by computer control, and the best focal length would be found by the algorithm. When the image of sample is in focus, the image will be the clearest. By finding the best focal length corresponding to each pixel position, we can convert to the height information for reconstructing the threedimensional surface profile.
\end{abstract}

Keywords: color, 3D object reconstruction, varifocal lens, focus algorithm

\section{Introduction}

Image processing technology is now very mature and widely used in industry. The most important part of image processing is to measure clear sample images [1]. Taking Automated Optical Inspection (AOI) as an example in image processing technology, automated optical inspection measured samples must have preciseness, and it is confirmed whether the image is in focus or whether it is accurate. Automated optical inspection is an essential foundation for automated biology, biomedical science applications and agent detection systems [23]. It requires accurate, fast, low cost and high resolution. In automated optical inspection, searching image in focus is not only the most important but also basic procedure, so foremost part is constructing focus algorithms in the experiment to determine whether the screened image is in focus. By mathematical processing, we can confirm with the sharpness which was calculated by the algorithm.

In fact, we are not able to know in advance whether the sample profile will exceed the depth of field, so we have to change the focal length between the sample and the measurement system. First of all, the method for changing the focal length is proposed to move the stage, and the motor is used to adjust the height of stage for changing the focal length. This method has worked well, but it is very time consuming. Therefore, we propose an improved method by using a varifocal lens to change the focal length between the sample and the measurement system [4]. The varifocal lens of this system is the core. It can adjust the focal length according to the change of the focal power. Benefit from the varifocal lens, the speed of whole measurement process was enhanced [5].

However, these optical measurement systems usually search the image in focus as primary and process the images as gray-scale, so we propose a 3D color surface profile reconstruction system with the varifocal lens as the core. Our experiment not only search the focus, but also uses the color space restores the color, and applies the measurement technique to reconstruct the $3 \mathrm{D}$ surface profile of the sample.
The optical measurement system uses the focus algorithm to calculate the sharpness of image. Since we adjusted the focal power of the optical measurement system, the best focal length at each pixel position could be found. The best focal length found is different at each pixel position, so we know that the focal power of photography system was adjusted to the corresponding value for getting the maximum sharpness. When the focal power corresponding to each pixel position is found, we can use the relation between focal power and displacement to convert the height information of the measured object and reconstruct the three-dimensional surface profile. This is beneficial when observing the shape of irregular surfaces are greater than the depth of field of the photographic system or where the sample is difficult to measure its surface profile [67].

\section{System setup}

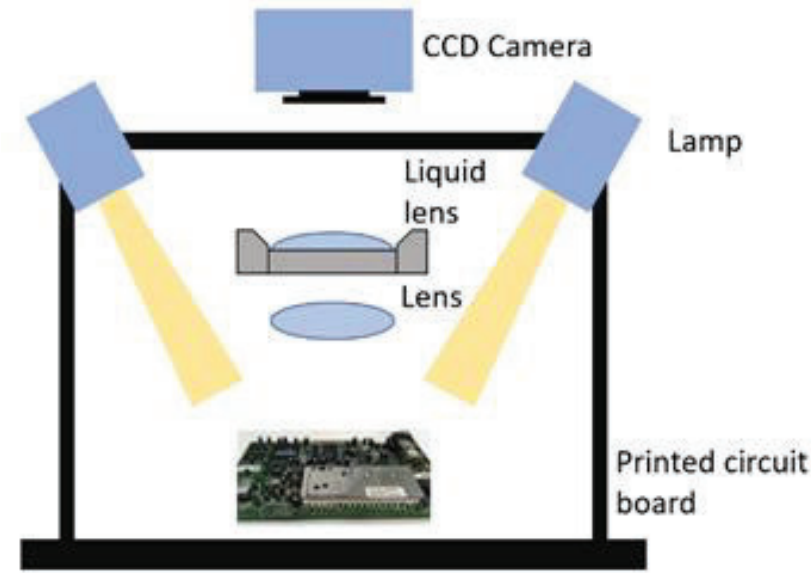

Figure 1. System setup for color 3D object reconstruction using varifocal lens 
Figure 1 display the construction of the proposed system, which is showing the color 3D object reconstruction using varifocal lens developed in this study. The proposed system consists of a CCD camera (EO-1312C-HQ LE), a varifocal lens, and a biconvex lens. The range of focal power is $3 \mathrm{dpt}$ to $11 \mathrm{dpt}$. Before light was reflected to the CCD camera, it would pass through the varifocal lens and biconvex lens to the sample. The CCD camera can capture hundreds of images in few seconds by change the curvature of the varifocal lens. In this system, we used focal power mode to adjust focal length. We used CCD camera capture images, biconvex lens to convert the magnification, so that we can transform the area covered by the system.

\section{Focus algorithm}

Those focus algorithms have been brought forward in the literature. The maximum output value of a focus algorithm will emerge from focused image and the value of output will decrease as defocus image. In the following order, there are four categories of algorithms in Derivative-Based Algorithms, Statistical Algorithms, Histogram-Based Algorithms and Intuitive Algorithms based on Sun Yu [8].

F-1 Tenenbaum Gradient: The algorithm used the Sobel mask in horizontal direction and vertical direction for the grayscale image, and then square the output values of the horizontal mask and the vertical mask.

$\mathrm{F}_{\text {Tenen }}=\sum_{\mathrm{y}=0}^{\text {Height }} \sum_{\mathrm{x}=0}^{\text {Width }} \mathrm{S}_{\mathrm{x}}(\mathrm{x}, \mathrm{y})^{2}+\mathrm{S}_{\mathrm{y}}(\mathrm{x}, \mathrm{y})^{2}$,

where $S_{x}(x, y)$ and $S_{y}(x, y)$ are the output values of the position $(\mathrm{x}, \mathrm{y})$ after the Sobel mask in the horizontal direction and the vertical direction are rotated back to the image. Sobel masks are typically used to detect horizontal and vertical edges, $S_{X}$ has a large response to horizontal edges, and $\mathrm{S}_{\mathrm{y}}$ is perpendicular to the horizontal. The following are the forms of the two masks

$\mathrm{S}_{\mathrm{x}}=\mathrm{I}(\mathrm{x}, \mathrm{y}) *\left[\begin{array}{ccc}-1 & 0 & 1 \\ -2 & 0 & 2 \\ -1 & 0 & 1\end{array}\right] \quad \mathrm{S}_{\mathrm{y}}=\mathrm{I}(\mathrm{x}, \mathrm{y}) *\left[\begin{array}{ccc}-1 & -2 & -1 \\ 0 & 0 & 0 \\ 1 & 2 & 1\end{array}\right]$

F-2 Auto Correlation:

The algorithm makes all pixels in the image multiply a neighboring pixel for summation, then subtract the summation of all pixels in the image multiply the adjacent two pixels.

$$
\begin{array}{r}
F_{\text {auto_corr }}=\sum_{\text {Height }} \sum_{\text {Width }} I(x, y) \cdot I(x+1, y) \\
-\sum_{\text {Height Width }} \sum_{I(x, y) \cdot I(x+2, y)}
\end{array}
$$

F-3 Entropy Algorithm: This algorithm supposed which focused images have more information than defocused images.

$$
F_{\text {entropy }}=-\sum_{\text {intensities }} P_{i} \cdot \log _{2}\left(P_{i}\right)
$$

and $P_{i}=h(i) / H \cdot W$ is the probability of a pixel with intensity $i$.
F-4 Image power: The algorithm sums up the squares of all the pixels in the image. If the brightness is strong, the calculation of sharpness will be large.

$$
F_{\text {power }}=\sum_{\text {Height Width }} \sum_{I(x, y)^{2}}
$$

\section{Experiments Results and discussion}

The following describes our experimental samples, experimental procedures, and experimental results. In the experiment we used printed circuit board as a measurement sample, and then adjusted the value of focal power from 3 to 11 $\mathrm{dpt}$ for taking 100 images, every $0.08 \mathrm{dpt}$ one shot, and the algorithm is added to calculate the sharpness.

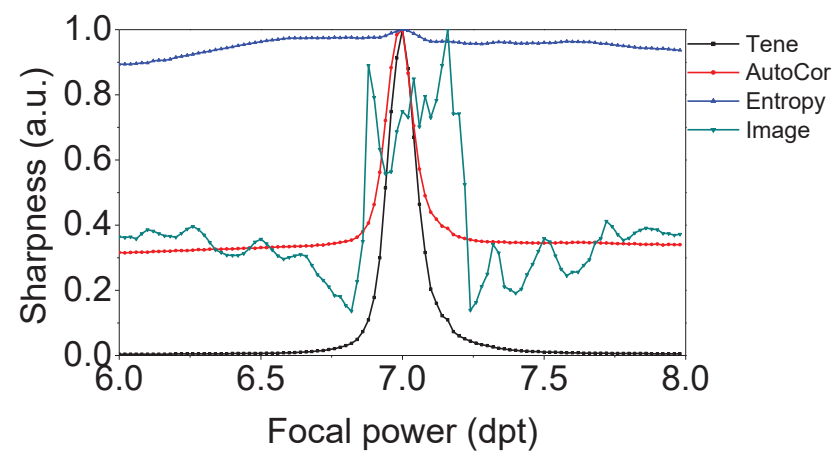

Figure 2. The sharpness curve of four algorithms

(a)

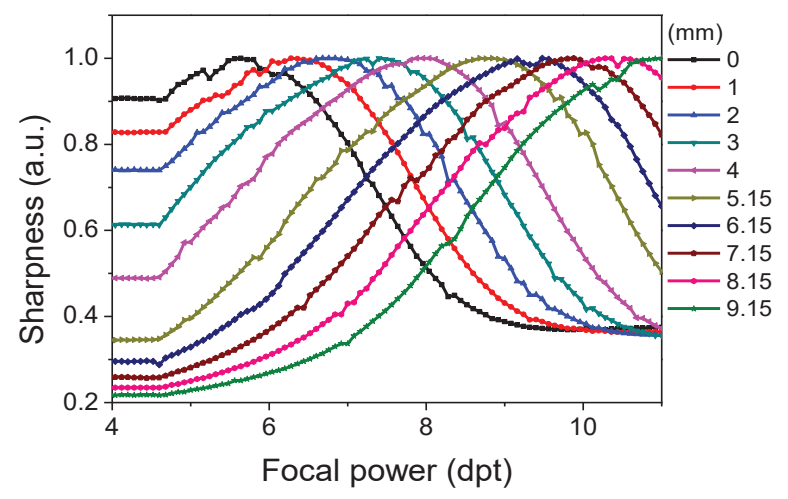

(b)

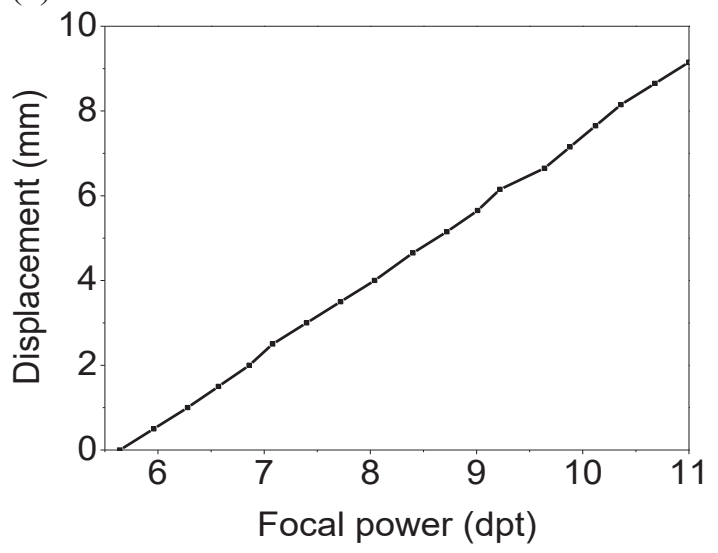

Figure 3. The sharpness curves of each height and relationship between displacement and focal power. 
First of all, we must to decide which algorithm we would use by experimental results. Considering the accuracy of the measurement, false Maxima and the FWHM. Obviously, we can find out the first algorithm which is without false maxima is sharper than others, so we chose to apply first algorithm to do the experiment of focus stacking. Establish the relationship between focal power and displacement is necessary for 3D object reconstruction. With focus algorithm, we can take out the maximum sharpness of each pixel position and get the corresponding focal power number. Using the relationship, we can convert the focal power into height information for construct the 3D surface profile of an object.

Then we started the experiment of color 3D object reconstruction and put the sample $\mathrm{PCB}$ on the stage to start measuring. Searching image in focus by our selected algorithm and recorded the sharpness of each pixel position under each focal length. Based on the technique of focus stacking, we can take out the focal length on each pixel position. With height information stacked from 2D images we can reconstruct the $3 \mathrm{D}$ surface profile of the sample.

(a)

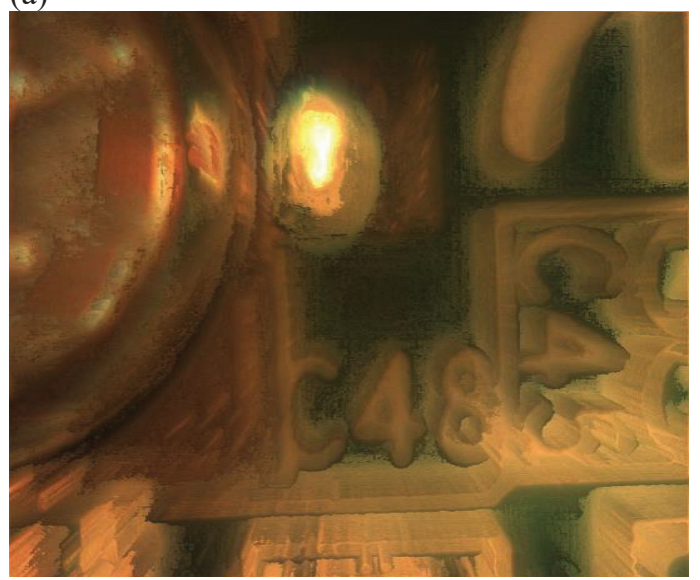

(b)

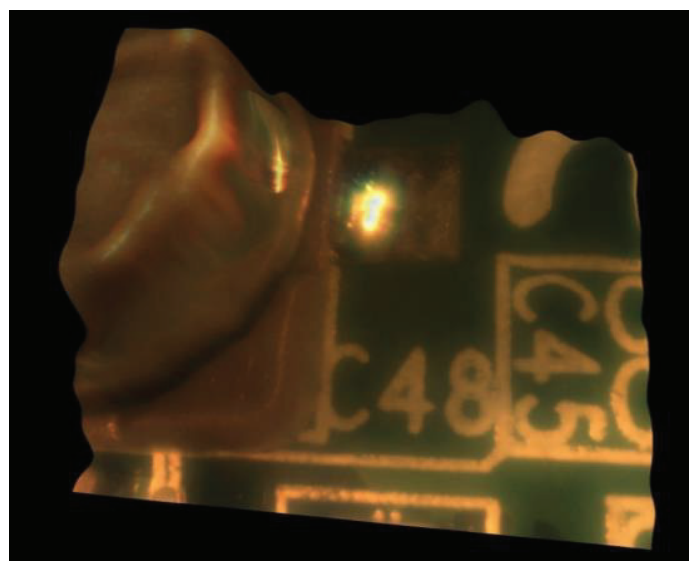

Figure 4. (a) DOF image (b) color 3D surface profile of software simulation.

\section{Conclusion}

Using the technique searching image in focus, we can calculate the sharpness of the image at each different focal length. According to the experimental results, the focal power can be reversed from the sharpness of the picture. Based on the focus stacking, the clear pixel position is stacked on the same image and the relationship between focal power and distance is brought in to count the surface profile of the sample.

\section{Acknowledgements}

This work was sponsored in part by the Ministry of Science and Technology Taiwan under contract number MOST 1072221-E-492 -024 -MY3, MOST 106-2622-E-492-004 -CC3 and MOST Add-on Grant for International Cooperation.

\section{References}

[1] Chen-Liang Fan et al., "Surface profiling measurement using varifocal lens based on focus stacking",IEEE Proceeding of I2MTC, 2017.

[2] Zheng-Jie Ye et al., "Rapid displacement sensor based on fitting scan", IEEE Proceeding of I2MTC, 2016.

[3] Bo-Rong Lu et al., " Thickness measurement of transparent object using a confocal displacement sensor", IEEE Proceeding of I2MTC, 2017.

[4] Wei Huang et al., "Evaluation of focus measures in multi-focus image fusion", Pattern Recognition Letters Vol. 28, Issue 4, Pages 493-500, 2007. IEEE Proceeding of I2MTC, 2015.

[5] Selina Casutt et al., "Fast and precise continuous focusing with focus tunable lenses", SPIE International Society for Optics and Photonics, Vol. 8982,Pages 89820Y-1 to Y-7, 2014.

[6] Bastien Billiot et al., "3D Image Acquisition System Based on Shape from Focus Technique”, Sensors, Pages 5040-5053, 2013.

[7] Douglas Clark et al., "A Rapid Image Acquisition Method for Focus Stacking in Microscopy", Microscopy Today, Pages 18-24, 2015.

[8] Sun Yu et al., "Autofocusing in Computer Microscopy: Selecting the Optimal Focus Algorithm”, Microscopy Research and Technique 65, page139 $-149,2004$.

[9] A. Santos, "Evaluation of autofocus functions in molecular cytogenetic analysis," Journal of Microscopy, 1997. 


\title{
Active optical systems with novel metal brightness amplifiers
}

\author{
Maxim V. Trigub*, Nikolay A. Vasnev, Vasiliy V. Vlasov \\ ${ }^{\mathrm{a}}$ V.E. Zuev Institute of Atmospheric Optics SB RAS, 1, Academician Zuev square, Tomsk, Russia
}

\begin{abstract}
The paper presents the results of the development, research and the use of novel metal brightness amplifiers for active optical systems creating. The copper bromide active media was used as an active filter. The construction of $\mathrm{CuBr}$ active element discharge tube was made with the external electrodes. It provided the possibility of the use of capacitance discharge for copper atoms excitation. The feasibility of imaging the processes of materials production and modification are discussed. To increase the maximum distance between object and imaging equipment are studied using two methods: laser monitor with an independent illumination source and the use of brightness amplifiers with the increased pulse of amplification.
\end{abstract}

Keywords: high speed imaging, active filtration, brightness amplifiers/

\section{Introduction}

The use of lasers allows to solve different problems of modern science and technology. Most often a laser is used as a source of energy which is focused on the object, for example in machining, medicine, materials production, additive and subtractive technology, imaging and etc. One of the modern way is to use the laser active optical system (LAOS) [1]. High speed imaging is one of the most popular method for studying the processes in the region of interaction of intense energy fluxes with matter. One of the problem of such processes visual diagnostics is the glare which is the result of the interaction. Optical and visual non-destructive testing is one of the most important method in the study and imaging of different fast processes. Active optical systems with metal vapor brightness amplifiers (which is called Laser monitor), have shown the high efficiency in filtering of the background radiation [2].

\section{Laser Monitor}

The layout of the laser monitor is shown on the fig 1. The laser monitor includes an brightness amplifier, optical system (Objective 1 and 2) and high speed camera. The high gain of the image brightness can be obtained with the use of $\mathrm{CuBr}$ active media. In the monostatic variant one active element (Brightness amplifier) is used to illuminate the object and to increase the intensity of the formed image

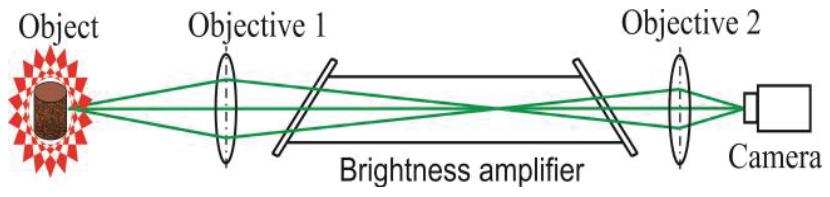

Figure 2. Monostatic laser monitor

In the (Bistatic laser monitor (fig. 2), two different elements are used. The laser is used to illuminate an object, and the brightness amplifier is used to amplify the image intensity. One of the challenge in this type of active optical system is the low efficiency of active elements. The GDT with external electrodes can be used to solve this challenge.

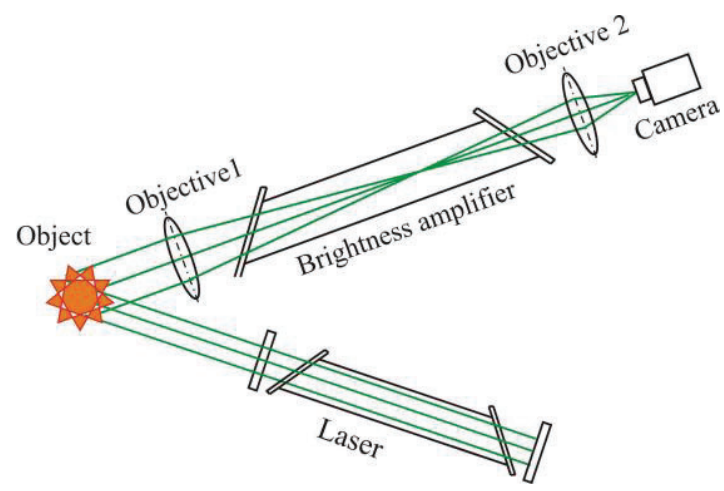

Figure 2. Bistatic laser monitor 
The high gain of the image brightness can be obtained with the use of $\mathrm{CuBr}$ active media. In the monostatic variant one active element (Brightness amplifier) is used to illuminate the object and to increase the intensity of the formed image. In the second variant (Bistatic laser monitor), two different elements are used. The laser is used to illuminate an object, and the brightness amplifier is used to amplify the image intensity. One of the challenge in this type of active optical system is the low efficiency of active elements. The GDT with external electrodes can be used to solve this challenge.

The construction of the GDT with external electrodes was developed in IAO SBR RAS [3]. Te construction excludes the contact of the electrodes material with a medium. The active element is made of quartz; the windows are used to output laser radiation. The electrodes are made of a metal foil wound on the outer wall of the GDT. The design allows you to change the equivalent capacity by connecting an additional capacitor ("second floor"). To excite the active medium, the capacitive barrier discharge is used in this type of GDT. It is formed by high-voltage pulse formed by a high speed commutator, for example, it can be thyratron. The use of independent heaters of containers with working substance $(\mathrm{CuBr})$ and the $\mathrm{HBr}$ generator allows to optimize the amplifying characteristics of this medium.

\section{Results and Discussion}

The work discusses the feasibility of developing active filters based on metal vapors for imaging processes blocked by the background glare. The system with external illumination and active filtration by the quantum filter has been created and used for test object imaging. It has been shown that the imaging method proposed in this work proves to be the most reliable to obtain the information about objects or processes in a real time mode using high PRF $\mathrm{CuBr}$ active media. The imaging results of different processes blocked from the observation by the intense background light are presented. The different types of active media were used as active quantum filters. For increasing spectral range of the imaging. The visualization results are shown on the fig 3 .
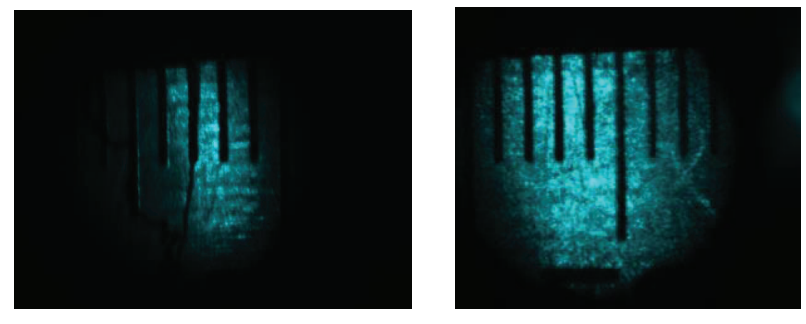

Figure 3. The imaging of the test object in the monostatic (a) and bistatic (b) laser monitors

The external glare was created by the arc with the brightness temperature about $5000 \mathrm{~K}$. The active filter allowed to describe the negative effect of this arc and make it possible to observe the object. The spatial resolution of the bistatic laser monitor is higher than the monostatic one, as it has been shown on the fig. 3. It provides by the external illumination source.

One of the feature of the active optical system is a high time resolution, It provides by the use high frequency active filters. In the work some fast processes were visualized by the laser monitor. Each frame was formed by the one pulse of amplification (about $40 \mathrm{~ns}$ ). It has allowed to study the processes of materials production and modification.

The authors are grateful to Professor of Tomsk Polytechnic University G.S. Evtushenko for discussion of the results.

\section{References}

[1] Chvykov, V. (2017). Laser active optical systems (LAOS) for industrial applications. Paper presented at the Optics InfoBase Conference Papers, Part F43-CLEO_AT 2017 doi:10.1364/CLEO_AT.2017.ATu3C.2

[2] Trigub M.V., Evtushenko G.S., Torgaev S.N., Shiyanov D.V., Evtushenko T.G. Copper bromide vapor brightness amplifiers with $100 \mathrm{kHz}$ pulse repetition frequency // Optics communications, 2016. Vol. 376, No 10, pp. 81-85

[3] Gubarev F.A., Shiyanov D.V., Sukhanov V.B., Evtushenko G.S. Capacitive-Discharge-Pumped $\mathrm{CuBr}$ Laser With $12 \mathrm{~W}$ Average Output Power // IEEE Journal of Quantum Electronics. 2013. V.49. No1. P.89-94. 


\title{
Phase analysis of light carrying optical vortex for refractive index sensing
}

\author{
Youngbin $\mathrm{Na}^{\mathrm{a}}$, Do-Kyeong Ko*a \\ ${ }^{a}$ Department of Physics and Photon Science, Gwangju Institute of Science and Technology, Gwangju, \\ 61005, Republic of Korea
}

\begin{abstract}
We present a highly sensitive refractive index (RI) sensor based on a phase analysis of light beam carrying an optical vortex. Because the phase angle of the vortex is proportional to the spatial azimuth angle, the rotated vortex phase can provide the RI of an optical medium. By using a spatial light modulator (SLM), we design a modified Mach-Zehnder interferometer for system stability. As a proof of concept, we measure the vortex phase and calculate the rotated angle according to the concentration of aqueous solutions. The use of the phase analysis and the stable interferometer allows precisely detecting the RI with high-resolution. In addition to this, we investigate changes in resolution and dynamic range over different path lengths.
\end{abstract}

Keywords: Interferometry, Refractometer, Optical vortices, Spatial light modulator, Resolution, Dynamic range

\section{Introduction}

Refractive index (RI) of optical media is a basic property, which is often employed to measure the concentration of liquids and detect its real-time change. Precisely measuring the concentration is an indispensable factor in research fields such as chemical industry, chemical analysis and fabrication of nanocomposite thin films. Recently, transparent conducting thin films have been getting much attention to develop efficient solar cells $^{1,2}$. For this reason, various groups have proposed measurement techniques with advantages of both highsensitivity and high-resolution ${ }^{3,4}$. However, they suffer from direct contact with samples, a weak tunability and fabricating nanoscale devices. To overcome these limitations, we propose an interferometric technique using light carrying optical vortex.

In this work, we first experimentally verify the stability of a modified Mach-Zehnder (MZ) interferometer by monitoring phase fluctuation. This result demonstrates the precision of the proposed technique. With the stable interferometer and optical vortex, we perform PSI experiment on six aqueous salt solutions with different concentration. In addition to this, we investigate changes in resolution and dynamic range by applying different path length of the sample.

*dkko@gist.ac.kr;

\section{Experimental setup and method}

As depicted in Fig. 1, the modified MZ interferometer based on a spatial light modulator (SLM) is designed to construct a stable system. After separation into two paths by a polarizing beam splitter, a transmitted horizontal beam becomes an object beam, and a reflected vertical beam acts as a reference beam. Because a parallel-aligned nematic liquid crystal SLM (PLUTO-VIS, Holoeye) is designed to modulate only a phase of the horizontal polarization, the vertical beam is reflected off without a modulation effect. On the other hand, the horizontal beam is changed into an optical vortex and diffracted by the fork hologram loaded into the SLM.

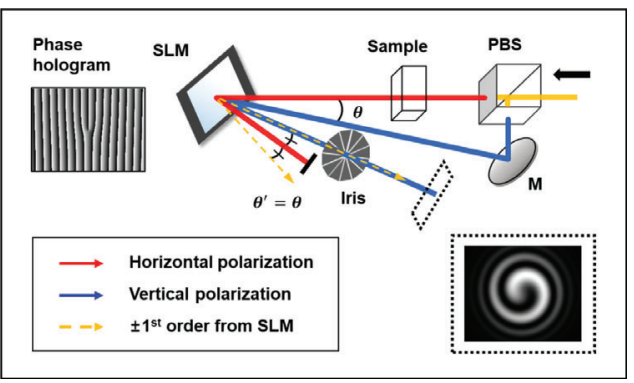

Figure 1. Schematic diagram of a modified MZ interferometer: PBS, polarizing beam splitter cube; M, mirror. The insets show a phase hologram for vortex generation and collinear alignment and (black dotted line) a spiral interference produced by the vortex and Gaussian beam. 
The incident vertical and horizontal beams converge at one point on the SLM. The object beam is dffracted $\left(+1^{\text {st }}\right.$ order), with the diffraction angle of $\theta^{\prime}$. When we select a proper grating period, the reflected referenc beam and the diffracted object beam collinearly propagate, i.e. $\theta^{\prime}=\theta$.

\section{Results and Discussion}

\section{System stability}

An ordinary Michelson interferometer is additionally used to compare the system stability. We measure vortex phases of an object beam without a sample. 20 sequential measurements are conducted with a time interval of 30 seconds. Figure 2(a) and (b) shows phase values at a randomly selected position in the retrieved phase map. While the Michelson interferometer shows unstable results, the modified MZ interferometer keeps the phase fluctuation low. Figure 2(c) depicts the statistical result of the phase fluctuation. The mean value are 0.393 and 0.0874, which demonstrates the robust stability and repeatability of the proposed system.
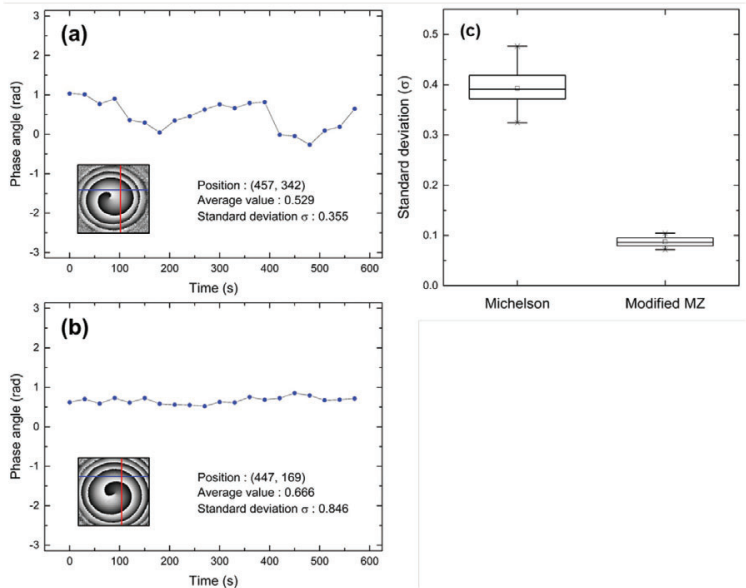

Figure 2. Stability test using phase fluctuation of (a) the Michelson interferometer and (b) the modified MZ interferometer and (c) statistical analysis from randomly chosen 30 positions. The each inset shows a reconstructed vortex phase and the measure position.

\section{Performance of the vortex refractometer}

A vortex phase and its rotated angle are used to determine the relative phase shift introduced by each concentration. We prepared six aqueous salt solutions as test samples with different concentration $(\%, w / w), 10.03$, $10.04,10.07,10.10,10.15$, and 10.25 , respectively. The probing beam passes through the solution with $2 \mathrm{~mm}$ path length. Figure 3 shows captured spiral interference patterns for each concentration. The vortex phases are obtained by the four-step PSI ${ }^{5}$, and the relative phase shift is extracted by the following equation ${ }^{6}$ :

$\theta=a r^{2}+b_{0}$,

where $a$ represents the rate at which the angle changes as its radius increases. $b_{0}$ is the phase shift corresponding to the starting angle of the vortex. Fitting result of the sample with $10.25 \%$ concentration is shown in Fig. 4(a) as an example. Here, we extracted the values along a phase minimum line, i.e. the black line. Figure 4(b) shows the values of $b_{0}$ versus concentration, and the slope determines the rate of change, $d b_{0} / d C$. A linear relation between $n$ and $C$ is estimated by the following equation:

$\Delta n k d=\Delta n \frac{2 \pi}{\lambda} d=\Delta b_{0}[\mathrm{rad}]$

where $n$ is the RI of the sample and $d$ is the path length in the medium. Thus, the relation $d n / d C$ is given by

$$
\frac{d n}{d C}=\frac{\lambda}{2 \pi d} \frac{d b_{0}}{d C}\left[\%^{-1}\right] .
$$
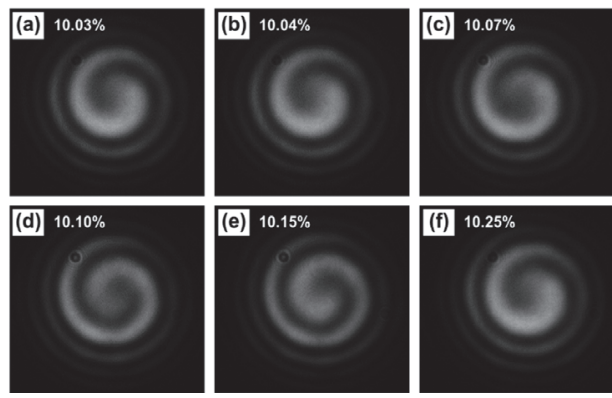

Figure 3. Captured interferograms from the six different concentration.

The difference of $0.01 \%$ corresponds to the RI of $\sim 1.95 \times 10^{-5}$ refractive index unit (RIU). The use of short wavelength and long path length can further improve the resolution as shown in the Eq. (2). From the equation, the phase fluctuation of 0.0874 measured in the stability test gives the detection limit of $\sim 4.13 \times 10^{-6}$ RIU, which demonstrates that the method using the vortex phase is a precise technique. 

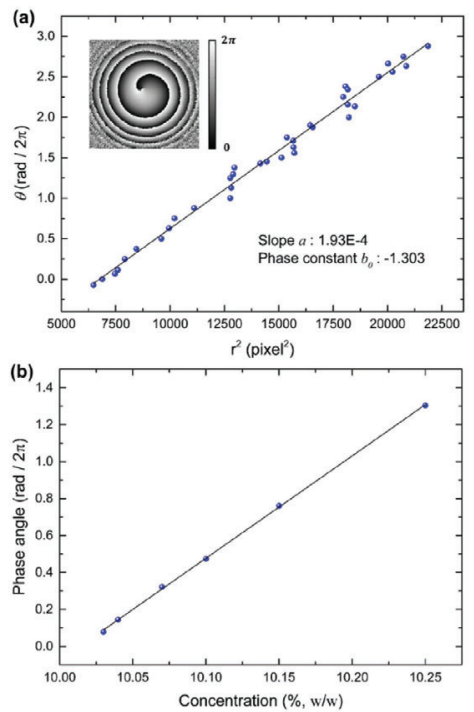

Figure 4. (a) Fitting results of the extracted minimum points for $10.25 \%$ and (b) calculated phase constant. The inset shows the retrieved vortex phase.
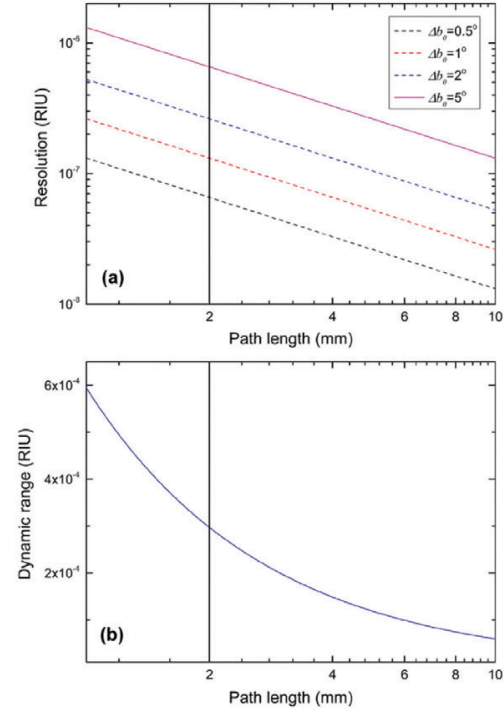

Figure 5. Performance of the proposed method at various path lengths. (a) Estimated resolution as a function of path length under different values of detection limit and (b) dynamic range as a function of path length. Our experimental condition corresponds to $2 \mathrm{~mm}$ path length and $\sim 5^{\circ}$ detection limit.

Finally, the performance of the proposed technique is displayed in Fig 5. Figure 5(a) depicts estimated resolution as a function of path length. Each color indicates different scenarios of the detection limit. Figure 5(b) shows the dynamic range of the method. A much smaller resolution can be achieved by lowering the detection limit, potentially up to $10^{-6}$ or even $10^{-7}$ RIU. We believe that it will be realized by adopting a nearly common-path interferometer.

\section{Conclusion}

In conclusion, we proposed and experimentally demonstrated the vortex based interferometric method for application to the RI sensor. The rotation in the phase profile and its differential measurement were utilized to precisely detect the RI change, and experimental results verified the possibility of a high-resolution refractometer. The resolution limit of the proposed technique will be improved further, up to $10^{-6}$ or even $10^{-7}$, by using the longer path length and enhancing the system stability. However, there is a drawback in the dynamic range due to the ambiguity of the phase. Thus, we will seek to break the fundamental limit on the dynamic range.

\section{References}

[1] S. Vadivel and G. Rajarajan, "Effect of Mg doping on structural, optical and photocatalytic activity of $\mathrm{SnO}_{2}$ nanostructure thin films," J. Mater. Sci. Mater. Electron. 26, 3155-3162 (2015).

[2] X. Xu, J. Bullock, L. Schelhas, E. Stutz, J. Fonseca, M. Hettick, V. Pool, K. Tai, M. Toney, X. Fang, A. Javey, L. Wong and J. Ager, "Chemical Bath Deposition of pType Transparent, Highly Conducting $(\mathrm{CuS})_{\mathrm{x}}:(\mathrm{ZnS})_{1-\mathrm{x}}$ Nanocomposite Thin Films and Fabrication of $\mathrm{Si}$ Heterojunction Solar Cells," Nano Lett. 16, 19251932 (2016).

[3] Z. Chen, L. Liu, Y. He and H. Ma, "Resolution enhancement of surface plasmon resonance sensor with spectral interrogation: resonant wavelength considerations," Appl. Opt. 55, 884-891 (2016).

[4] Z. Ding, T. Lang, Y. Wang and C. Zhao, "Surface Plasmon Resonance Refractive index sensor based on Tapered Coreless Optical Fiber Structure," J. Light. Technol. 35, 4734-4739 (2017).

[5] G. Lai and T. Yatagai, "Generalized phase-shifting interferometry," J. Opt. Soc. Am. A 8, 822-827 (1991).

[6] Y. Xu, J. Sun, W. Walasik and N. Litchinitser, "Probing metamaterials with structured light," Opt. Express 24, 26249-26254 (2016). 


\title{
SMS and FBG interrogation for measurement of temperature and strain using OTDR
}

\author{
Koustav Dey*a, Sourabh Roy ${ }^{\mathrm{a}}$, M.Sai Shankarª, B.Ramesh Kumar ${ }^{\mathrm{b}}$, P.Kishore ${ }^{\mathrm{a}}$ \\ a Department of Physics, National Institute Of Technology, Warangal 506 004, Telangana, India \\ ${ }^{\mathrm{b}}$ Institute for Plasma Research, Gandhinagar 382 428, Gujrat, India
}

\begin{abstract}
Here, we demonstrate the interrogation technique of Fiber Bragg grating (FBG) using single mode-multimode-single mode (SMS) with the help of optical time domain reflectometer (OTDR) for temperature and strain measurement. A temperature range of $20^{\circ} \mathrm{C}-200^{\circ} \mathrm{C}$ and strain range of $100 \mu \varepsilon-2020 \mu \varepsilon$ has been measured with a linearity of 0.994 with the proposed scheme. Our experimental result shows that this sensor has a temperature and strain sensitivity of $5.03 \mathrm{pm} /{ }^{\circ} \mathrm{C}$ and $0.4 \mathrm{pm} / \mu \varepsilon$ respectively. The proposed scheme offers low cost and better sensitivity with respect to existing methods.
\end{abstract}

Keywords: FBG, OTDR, SMS, interrogation, sensor

\section{Introduction}

In the era of boom of the optical fiber sensing technology, the multimode interference (MMI) effect has been intensively investigated with Single-modemultimode-single-mode (SMS) fiber structure as one of the typical representatives of the underlying operation principle of $\mathrm{MMI}^{1}$. The change in temperature and strain will affect the refractive index and dimensions of the fiber core and cladding which in turn affect the interference between modes in the MMF section that causes output response changes ${ }^{2}$. Alongside FBG is a wavelength coded sensing device which needs an interrogation system for essential conversion of optical power to wavelength information ${ }^{3}$. Hence the combining sensing outputs from FBG and SMS signals are monitored using OTDR. The obtained results of strain and temperature from this proposed scheme perform better and show enhanced sensitivity with respect to existing methods.

\section{Experimental Details}

The SMS fiber structure is fabricated using a commercial fusion splicer (Fujikura-60S). A multimode fiber (50/125 $\mu \mathrm{m})$ section is sandwiched between two step index single mode fibers $(9 / 125 \mu \mathrm{m})$ axially. The OTDR (JDSU MTS 8000 series) is used to detect the event induced by the temperature and strain change on FBG.

*physicskoustav@gmail.com; phone +91 9440218973
The OTDR measures the attenuation of reflected light or return loss and the location from where the light is being reflected within $5 \mathrm{~km}$ range of optical fiber network with resolution of $0.001 \mathrm{~dB}$. For temperature sensing, we have kept the FBG inside an oven which is again connected to the dimmer stat for controlling the temperature with time. For strain measurement, we have pasted the FBG on the simple beam cantilever using high-temperature instant adhesive. Here we have investigated the attenuation loss of an FBG of central wavelength $1528 \mathrm{~nm}$, which lies in the ripple free slope region of the SMS using the interrogation technique of SMS and FBG with the help of OTDR. The power loss is a measurement of variation of temperature and strain separately and also for variation of temperature at discrete constant loads (50 gms to $1000 \mathrm{gms}$ with an increment of $50 \mathrm{gms}$.) in the range $20^{\circ} \mathrm{C}-200^{\circ} \mathrm{C}$ using OTDR at constant room temperature. As the FBG peak follows the linear slope region of SMS, it is expected that the response of the OTDR also to be linear which is confirmed from the obtained results, that showing good linearity of $\sim 0.994$. Our scheme of interrogation is expected to be robust, cheap and more efficient for simultaneous measurement of temperature and strain with good sensitivity. Our experimental result shows that this sensor has a temperature and strain sensitivity of $5.03 \mathrm{pm} /{ }^{\circ} \mathrm{C}$ and $0.4 \mathrm{pm} / \mu \varepsilon$ respectively. From which we can measure the temperature and strain simultaneously. The 
OTDR is connected serially to the FBG and SMS as shown in Fig. 1.

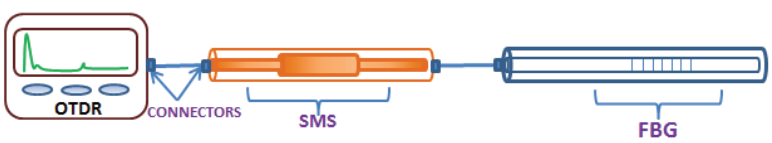

Figure 1. Schematic diagram of experimental set-up

\section{Results and discussion}

The graphs between power loss vs. temperature and power loss vs. strain have been drawn. We have also drawn the power loss vs. temperature graph at different loads.

For temperature and strain measurement we have plotted the graphs between wavelength shift vs. temperature and wavelength shift vs. strain.
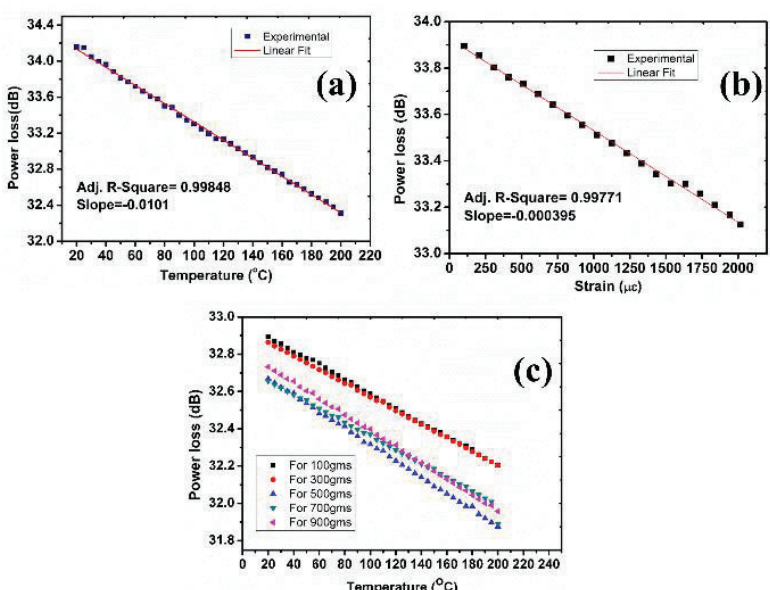

Figure 2. (a) Power loss vs. temperature, (b) Power loss vs. strain and (c) Power loss vs. temperature at different loads.
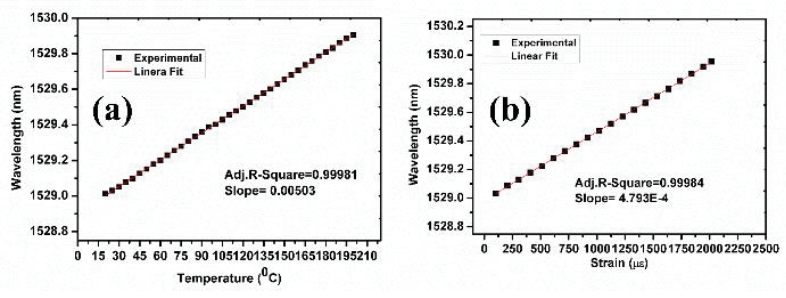

Figure 3. (a) Wavelength Shift vs. temperature and (b) Wavelength shift vs. strain.
The relationship between the central wavelength, power loss, temperature and strain can be expressed using a matrix as follows:

$$
\begin{gathered}
\left(\begin{array}{c}
\Delta \lambda \\
\Delta P
\end{array}\right)=\left(\begin{array}{ll}
K_{11} & K_{12} \\
K_{21} & K_{22}
\end{array}\right)\left(\begin{array}{c}
\Delta T \\
\Delta \varepsilon
\end{array}\right) \\
=\left(\begin{array}{cc}
5.03 p m /{ }^{\circ} \mathrm{C} & 0.4 p m /{ }^{\circ} \mathrm{C} \\
-10.01 p m /{ }^{\circ} \mathrm{C} & -0.39 p m /{ }^{\circ} \mathrm{C}
\end{array}\right)\left(\begin{array}{l}
\Delta T \\
\Delta \varepsilon
\end{array}\right)
\end{gathered}
$$

Where $\mathrm{K}_{11}, \mathrm{~K}_{12}, \mathrm{~K}_{21}$, and $\mathrm{K}_{22}$ are the matrix coefficients for wavelength/temperature, wavelength/ strain, power /temperature, and power/strain respectively. To obtain the matrix coefficients, a linear fit is applied to each measured characteristics in Figs.2(a)-(b) and 3(a)-(b).It is noted that all the data in the figures display good linearity with $\mathrm{R}^{2}>0.992$.

\section{Conclusions}

In conclusion, we have reported an experimental study of a novel and cost-effective interrogation technique for measuring temperature and strain with the help of OTDR. As the FBG peak follows the linear slope region Of the SMS, it is expected that the response of the OTDR also to be linear which is confirmed from the obtained results. We achieved the linearity $\sim 0.994$. Obtained good linearity about 0.994 from the large range measurement of temperature and strain confirmed the better performance of this scheme than previously existing methods. The sensor has an experimentally demonstrated temperature sensitivity of $5.03 \mathrm{pm} /{ }^{\circ} \mathrm{C}$ and strain sensitivity $0.4 \mathrm{pm} / \mu \varepsilon$. As OTDR is a very cheap and widely available instrument in the market, so this proposed method is very cost effective.

\section{References}

[1] P.Wang, M.Ding, L.Bo,C.Guan, Y.Semenova, Q.Wu, G. Farrell, and G.Brambilla, "Fiber-tip high temperature sensor based on multimode interference," Optics letters 38, 4617-4620 (2013)

[2] A.M.Hatta, K. Indriawati, T.Bestariyan, T.humada and sekartedjo, "SMS fiber structure for temperature measurement using an OTDR," photonic sensors 3, $262-266$ (2013) 
[3] Qiang wu,Yuliya semenova, Pengfei wang and Gerald Farrell, "High sensitivity SMS fibre structured based refractometer-analysis and experiment," Optic express 19,7937-7944 (2011)

[4] O frazao, J P Carvalho, L A Ferreira, F M Araujo and $\mathrm{J}$ L santos, "Discrimination of strain and temperature using Bragg gratings in microstructured and standard optical fibers," meas.Sci.technol. 16, 2109-2113 (2005)

\section{Acknowledgement}

The author is highly grateful to BRNS (RS-872-GoIBRNS-(DAE)-SR-PHYSICS (2017-18)) for their funding's. 


\title{
Development of an Anamorphic Liquid-pressure Varifocal Lens*
}

\author{
Ryoichi Kuwano*a $^{\text {a }}$, Makoto Hino ${ }^{\mathrm{a}}$, Tsuyoshi Tokunaga ${ }^{\mathrm{b}}$, Sho Morita ${ }^{\mathrm{b}}$ and Yukitoshi Otani ${ }^{\mathrm{c}}$ \\ a Department of Mechanical Systems Engineering, Hiroshima Institute of Technology, \\ 2-1-1 Miyake, Saeki-ku, Hiroshima, 731-5193, Japan \\ ${ }^{b}$ Department of Innovative Mechanical and Electronic Engineering, Chiba Institute of Technology, \\ 2-17-1 Tsudanuma, Narashino, Chiba 275-0016, \\ ${ }^{c}$ Utsunomiya University Center for Optical Research \& Education (CORE), \\ 7-1-2, Yoto, Utsunomiya-city, Tochigi, 321-8585, Japan
}

\begin{abstract}
This paper details the study of an anamorphic liquid-pressure varifocal lens that can be used to adjust the energy density of a laser in the three dimensions of the $x-y$ plane and the optical $(z)$ axis direction. In the study, the optical characteristics of the lens and the deformation of the elastic film on the $x$ and $y$ axes were determined. Measurement to establish the focal length of the lens at a circular beam spot at the focal point produced values in the range from 580 to $142 \mathrm{~mm}$. The spot was characterized by an elliptical form with a varying aspect ratio before and after the focal position, and an anamorphic effect was observed. The lens is expected to be useful in laser processing of optical elements for joining dissimilar materials in the fields of engineering plastics and metals.
\end{abstract}

\section{Introduction}

Lenses are essential for the handling of light in optical application systems, but their solid nature produces inflexibility in the area of adjusting focal length for different applications; either the lens or the target material must be physically moved to change the focal position. The ability to change the focal length of the lens itself would speed up focusing operation and enable system downsizing.

Energy density, which involves the relationship between laser output and spot diameter, is an essential consideration in laser-based material processing. A variety of laser processes are conducted without sharp focus, including applications in laser welding, laser-based formation and surface modification. Such processing is characterized by problems such as energy loss and difficulty in adjusting heating and cooling times as a result of laser irradiation other than in the scanning direction due to lens-related beam defocusing. Against such a background, the availability of a function to allow energy density adjustment in the processing direction on the beam irradiation plane can be considered useful as an optical element in laser processing.

The authors previously reported on a liquid pressure- controlled variable-focus lens [1,2], and other such lenses have also been proposed [3 - 8]. However, issues remain to be addressed with these lens elements; in particular, they are unsuitable for lasers with near-infrared wavelengths and for high-power laser processing.

This paper details the study of an anamorphic liquidpressure varifocal lens that can adjust the energy density of a laser in the three dimensions of the $x-y$ plane and the optical ( $z$ ) axis direction. To support adjustment of the spot shape on the plane, a rectangular form was adopted for the peripheral support plate used to fix the elastic film of the proposed variable-focus lens and create an anamorphic refractive surface. The results of measurement to determine the optical characteristics of the lens and deformation of the elastic film on the $\mathrm{x}$ and $\mathrm{y}$ axes are reported.

\section{Anamorphic liquid-pressure varifocal lens}

The structure of the proposed lens (Figure 1) involves the use of a liquid-filled cavity, sealing with an elastic film and a transparent plate. The focal length is varied by changing the shape of the lens via liquid injection and discharge. Pressure on the inside of the lens causes deformation of the elastic film (the refractive surface) depending on the shape of the peripheral support plate. In

*kuwano@,me.it-hiroshima.ac.jp; phone +81-82-921-3121 


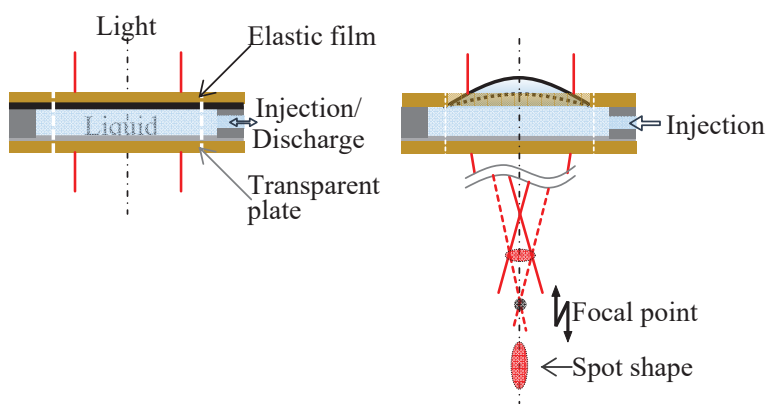

(a) Initial state

(b) Variable in convex lens

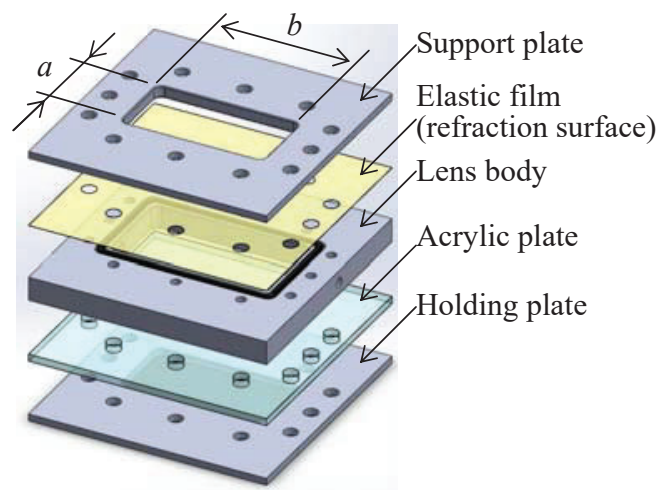

(c) Structure

Figure 1. Anamorphic liquid-pressure varifocal lens characteristics..

this study, a rectangular plate with a length of $a \mathrm{~mm}$ and a width of $b \mathrm{~mm}$ was used. The aspect ratio was $\mathrm{m}=a / b$, and the focal length and spot shape of the lens could be varied by adjusting its form with liquid pressure.

\section{Experimental results}

The optical characteristics of the lens and the deformation of the elastic film on the $x$ and $y$ axes were determined using the experimental setup shown in Figure 2. The resolutions of the pressure sensor and laser displacement meter were $1 \mathrm{kPa}$ and $10 \mu \mathrm{m}$, respectively.

Figure 3 shows the displacement of the lens center observed when pressure on the lens was increased in increments of $5 \mathrm{kPa}$. To ensure that the film (the refracting surface) was within the range of elastic deformation, atmospheric pressure was reassumed after determination of displacement before the next pressure value was set. The height of the lens center increased with pressure. Continuous pressurization from $0 \mathrm{kPa}$ at

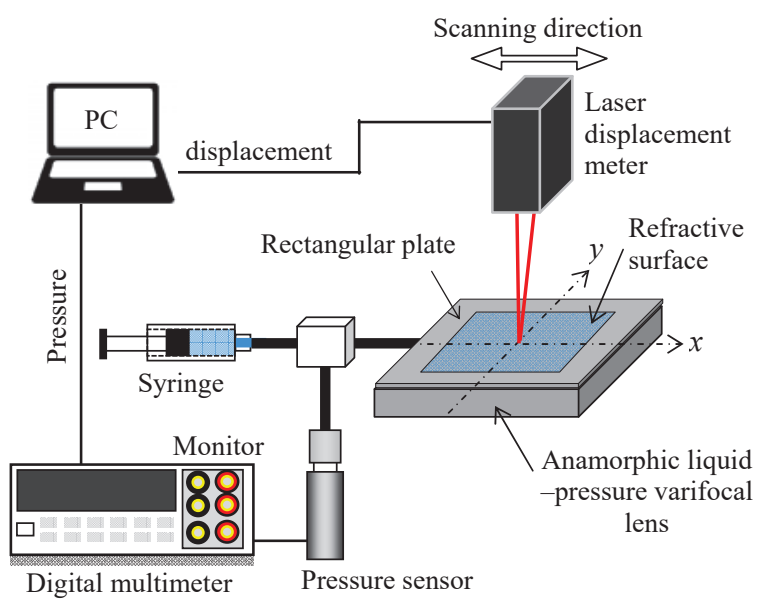

Figure 2. Experimental setup for determination of lens characteristics.

increments of $5 \mathrm{kPa}$ resulted in a lens-center displacement of $7.1 \mathrm{~mm}$ at $80 \mathrm{kPa}$. However, wrinkles appeared in the film with refraction surface values exceeding $20 \mathrm{kPa}$, and this value was accordingly taken as the limit of the elastic deformation range.

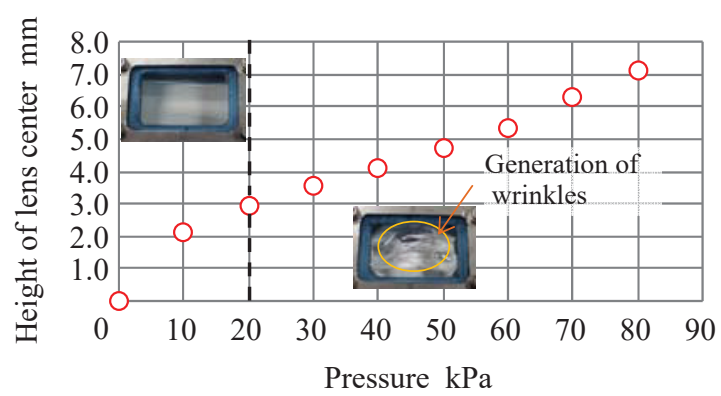

Figure 3. Relationship between pressure and height of lens center.

Figure 4 shows the relationship between pressure and focal length. The focal position was defined as that in which the beam spot was circular. The focal length was measured as the distance between this position and the end face of the lens. Its value decreased with higher pressure, and was $105 \mathrm{~mm}$ at $30 \mathrm{kPa}$. The focal length at $20 \mathrm{kPa}$ as the upper limit of effective pressure was 143 $\mathrm{mm}$. At pressure values from 0 to $8 \mathrm{kPa}$, the focal length varied from 582 to $240 \mathrm{~mm}$. Based on this observation, 


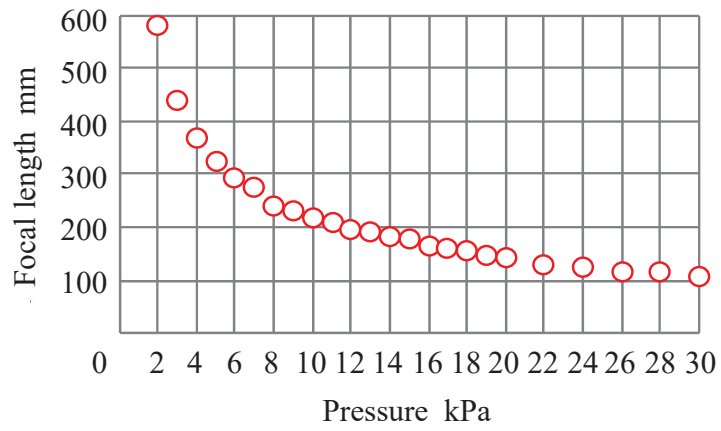

Figure 4. Relationship between pressure and focal length.

the anamorphic liquid-pressure varifocal lens can be considered suitable for use with long focal ranges.

Figure 5 shows the results of measurement to determine displacement of the elastic film on the $x$ and $y$ axes as the pressure was changed in $10 \mathrm{kPa}$ increments. The symmetry of the lens remained favorable, as shown in Fig. 5. Film deflection was proportional to liquid pressure, and displacement was consistent with the function of the fourth order with respect to position $(x, y)$.

Figure 6 shows the spot shape with a pressure value of $10 \mathrm{kPa}$; (b) shows a circular spot with a diameter of 6.8 $\mathrm{mm}$ at a focal length of $218 \mathrm{~mm}$, while (a) and (c) show the spot shape $\pm 100 \mathrm{~mm}$ from this position. Both shapes are ellipses, which indicates rotation of $90^{\circ}$ to the focal position.

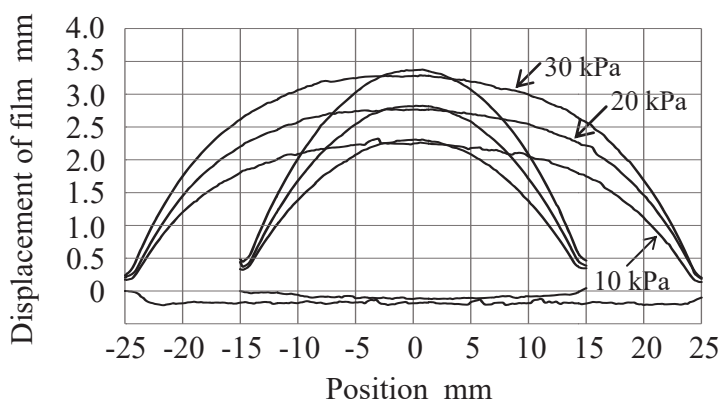

Figure 5. Axial displacement in relation to pressure on the elastic film.

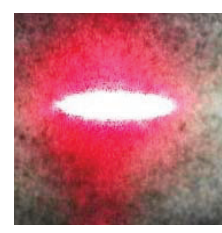

(a) Before the focal position $(\mathrm{z}=-100 \mathrm{~mm})$

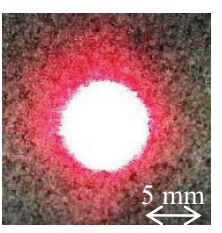

(b) Focal position (c) Behind the focal $(z=0 \mathrm{~mm}) \quad$ position $(\mathrm{z}=+100 \mathrm{~mm})$
Figure 6. Lens-related spot shape variation.

\section{Conclusions}

This paper details the study of a lens allowing focal position/spot shape changes with a structure incorporating liquid and an elastic film. The variable spot shape generates an anamorphic refracting surface, with the elastic film of the lens used as a rectangular peripheral support. The spot is an ellipse with different aspect ratios and $90^{\circ}$ rotation before and after the focal point. The effective focal length of the lens exceeded $200 \mathrm{~mm}$. It is considered suitable for use with optical elements in laser diode usage, such as in application with an output of several hundred $\mathrm{W}$ without the need for converging properties. In the future work, the authors plan to apply the lens to the task of joining dissimilar materials in the fields of engineering plastics and metals.

\section{References}

[1] R. Kuwano, T. Tokunaga, Y. Otani and N. Umeda : Opt. Rev. 12, 5(2005) : 405-408..

[2] R. Kuwano, T. Tokunaga, S. Morita, Y. Okane and Y. Otani. MATEC Web of Conferences, 32, 07003 (2015) : p.1-p.3.

[3] M. C. King and D. H. Berry : Appl. Opt. 9, (1970):2035.

[4] S. Sato : Jpn. J. Appl. Phys. 18(1979) 1679.

[5] N. Sugiura and S. Morita : Appl. Opt. 32, 22 (1993) 4181.

[6] T. Kaneko, I. Kanayama, T. Itogaki, T.Hattori : JSPE semestrial meeting spring, 3 (1996) (in Japanese).

[7] D. Y. Zhang, V. Lien, Y. Berdichevsky, J. Choi and Y. H. Lo : Appl. Phys. Lett, 82, 19(2003) 3171.

[8] B. H. W. Hendriks, S. Kuiper, M. A. J. van As, and C. J. Renders : Opt. Rev. 12, 3 (2005) 255. 


\title{
Digital holographic analyzer of optical fiber inhomogeneity at the soldering region
}

\author{
Bogdan Sokolenko*a, Andrey Prisyajniuk ${ }^{\mathrm{a}}$, Dmitrii Poletaev ${ }^{\mathrm{a}}$, Nataliya Shostka ${ }^{\mathrm{a}}$, Ismail Ismailov ${ }^{\mathrm{a}}$ \\ aDepartment of General Physics, V.I. Vernadsky Crimean Federal University 4 Vernadsky Avenue, \\ Simferopol, 295007, Russia
}

\begin{abstract}
In the present research, a digital lens free holographic analyzer of defects in optical fibers was developed. This technique applicable for robust imaging of optical inhomogeneity in internal structure of phase-amplitude objects that are difficult to observe with standard optical microscopes. Proposed portable holographic in-line system is used for observing for instant analysis of manufacturing defects and for illustration of the capabilities of this system, several optical soldered fibers were analyzed: with obvious defects up to 10 microns mismatch and in regular conditions of soldering.
\end{abstract}

Keywords: digital holography, defects, optical fiber,

\section{Introduction}

Today, holography is firmly established as a tool for scientific research [1]. Its practical application has a sufficiently effective implementation due to the use of computer software. In particular, the methods and technologies of digital holography are used in the tasks of particle research, in the protection of counterfeiting and falsification of goods, in optical microscopy [2, 3]. Holographic research methods are used in the study of processes that occur in deformed environments, in the study of objects of different nature and spatial scale, in increasing the resolution of the holograms [4].

The appearance of holography increased the importance and role of light as a tool for storing and transmitting information. For example, on a hologram, several images (signals) may be captured, which, if restored, will not reveal mutual interference. In flat holograms with such an exposure, cross-images are created, but they do not interfere with the main image, in turn, volume holograms completely exclude the appearance of interference images.

However, the most clearly informational essence is manifested in the digital hologram. The digital holography has opened an access to modeling and synthesizing the intensity and phase of the electromagnetic field wave [5], which serves as an alternative to analog methods. Numerical methods implemented on computers are ahead of the classical ones by means of high precision processing, the processing algorithm itself and the simplicity of changing its parameters, the availability of results, the possibility of complex non-linear and logical transformations and also the simplicity of the interaction at any stage processing. The basis of the holographic method is its ability to quickly process multidimensional information and the ability to create devices with large memory capacities.

The digital holography is a subject of growing interest and finds application in both spatial and biomedical visualization, including metrology. The use of holographic research methods often requires the use of a large number of optical elements.

The article presents a unique portable lensless digital holographic set-up for qualitative and more accurate analysis of the properties of objects during deformation and also determining the shape of objects, visualizing phase distributions with high spatial resolution.

\section{Digital holographic analysis of optically transparent phase objects \\ 1. Theoretic background}

Numerical reconstruction of a digitally recorded hologram is carried out in accordance with the scalar 
diffraction theory in the Frenel approximation for the Rayleigh-Sommerfeld diffraction integral. Restored Diffracted Field $Q(\xi, \eta)$ in the image plane $(\xi, \eta)$ at a distance $d$ from the hologram plane can be represented in the paraxial approximation as follows:

$$
\begin{aligned}
& Q(\xi, \eta)=\frac{1}{i \lambda d} \exp \left(i \frac{2 \pi}{\lambda} d\right) \times \\
& \times \int_{-\infty}^{\infty} \int_{-\infty}^{\infty} o_{2}(x, y) I(x, y) d x d y \times \\
& \exp \left[i \frac{\pi}{\lambda d}\left[(\xi-x)^{2}+(\eta-y)^{2}\right]\right] d x d y .
\end{aligned}
$$

Equation (1) serves as the starting point for the numerical reconstruction of images in digital holography in the paraxial approximation, with which the $x$ and $y$ coordinates, as well as the corresponding $\xi$ and $\eta$ are small in comparing to the distance $d$. From equation (1) it can be seen that the resulting field is determined by a two-dimensional Fourier transform of the product of the intensity distribution in the hologram $I(x, y)$, complex

amplitude of the reference wave $o_{2}(x, y)$ and quadratic phase function of spatial wave propagation:

$$
w(x, y)=\exp \left[i \frac{\pi}{\lambda d}\left(x^{2}+y^{2}\right)\right] .
$$

Note that integral (1) is a convolution integral for the first two factors and function (2). Intensity $I(x, y ; d)$ and phase $\varphi(x, y ; d)$ of reconstructed images can be obtained from the complex field $Q(\xi, \eta)$ calculated at a distance $d$ using the following relations:

$$
\begin{aligned}
& I(x, y ; d)=|Q(x, y)|^{2}, \\
& \varphi(x, y ; d)=\operatorname{arctg}\left(\frac{\operatorname{Im}(Q(x, y))}{\operatorname{Re}(Q(x, y))}\right)=\arg (Q(x, y)) .
\end{aligned}
$$

Phase values $\varphi(x, y ; d)$, which was obtained by this formula correspond to a discontinuous function with a region of variation within the interval $[-\pi, \pi]$. One of the well-known phase unwarping algorithms can be applied to restore a continuous unfolded image of phase values.

\section{Experimental results and discussion}

The principle of the in-line configuration is that the laser light, passing through sample, splits into the two areas: diffracted by the sample and reference beam, without phase perturbations. Thus, interference of two beams is recorded in the plane of the CMOS matrix. It is obvious that the sample in this scheme must be transparent, otherwise only the shadow of the sample will be recorded. This imposes significant limitations on research, but it is easy to work with this configuration for analysis of optically transparent phase objects as optical fibers.

In the modern fiber optic industry, there are difficulties in controlling the quality of optical fibers associated with the inability of optical microscopy methods to determine refractive index fluctuations. With the help of the refractive index gradient, one can find out about defects on the core and the cladding of the optical fiber. From this point of view, the most appropriate methods for this task are digital holographic microscopy and tomography.

An urgent task is the creation of a portable holographic installation for the study of defects on an optical fiber in real time. The portable digital non-slip holographic installation described above, configured to the axial configuration, was used to determine defects in the welded joint of two of the same type fibers. Special fiberoptic blanks were made, on one of which an obvious displacement of the welded joint was created, and the other was created under normal conditions. In Figure 1, we may notice clearly observable defects on a wellquality welded joint in standard soldering regime.

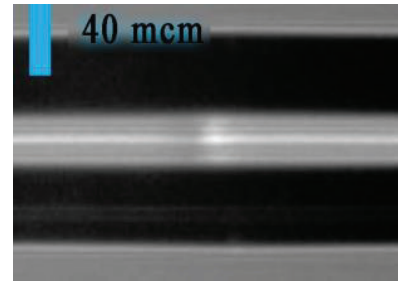

a

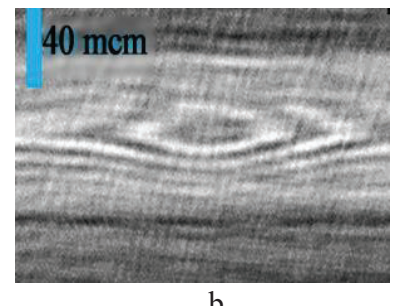

b
Figure 1. The area of soldered optical fiber conjunction in normal mode: a) the optical wild-field microscopy image and b) reconstructed image of the same place from digital hologram. 
Defects of the welded joint are also observed on the welded joint without visible displacement of the fibers relative to each other in image received from microscope.

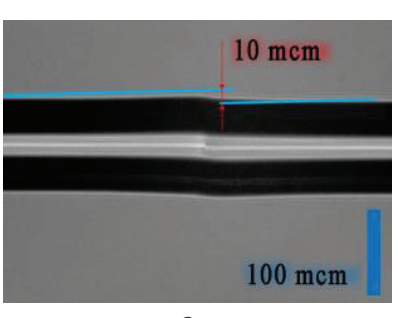

a

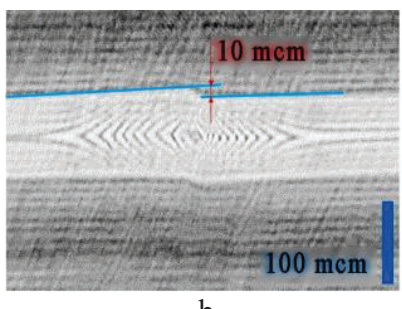

b
Figure 2. The area of soldered optical fiber conjunction in manual mode with induced misalignment of fibers: a) the optical wild-field microscopy image and b) reconstructed image of the same place from digital hologram.

Thus, digital holographic analysis can be used for defects imaging in welded joints of optical fibers. Depending on the goals, this technique can be broaden and adapted for inspection of phase objects and structural analysis.

\section{Conclusion}

Digital holography is based on the fundamental principles of coherent optics, and modern achievements of electronic equipment and computer technologies. At the same time, there are broad possibilities for the study of various objects by optical methods with a resolution determined by a small wavelength of optical radiation, the characteristics of the optical system and a recording system based on multi-element radiation detectors.

For the practical use of digital holography methods, it is necessary to take into account the peculiarities of the physical process of digital holographic recording and computer reconstruction of a hologram image. Recording schemes for digital holograms, as a rule, contain a large number of optical elements that introduce unwanted corrections into the resulting image. In addition, there is a need to develop portable and easy modified systems.

In this work, a digital lensless holographic device was developed, which can investigate optical inhomogeneity in phase objects, which are transparent and onerously observable with an optical microscope.

\section{Acknowledgments}

This work was supported by the V. I. Vernadsky Crimean Federal University Development Program for
2015 - 2024 within the framework of Grant support for young researches VG18/2019.

\section{References}

[1]. Hariharan, P., [Basic Of Holography]. Cambridge University Press, New York: School of Physics, 94-119 (2002).

[2]. Cox, S., Rosten, E., Monypenny, J., JovanovicTalisman, T., Burnette, D.T., Lippincott-Schwartz, J., Jones, G.E. and Heintzmann, R.," Bayesian localization microscopy reveals nanoscale podosome dynamics" Nature Methods 9(2), 195-200 (2012).

[3]. Myung, K. K., "Principles and techniques of digital holographic microscopy" SPIE Reviews 1(1), 018005-7 (2010).

[4]. Latychevskaia, T., Fink, H.-W., "Resolution enhancement in digital holography by self-extrapolation of holograms Optics Express 21(6), 2013.

[5]. Schnars, U., Juptner, W. [Digital Holography], Springer-Verlag Berlin Heidelberg, New York, 101-123 (2005). 


\title{
Design of high-FOV automatic optical inspection lens for linear sensor with different magnification
}

\author{
Wei-Jei Peng, Cheng-Fang Ho, Ting-Ming Huang*, Yuan-Chieh Cheng, Fong-Zhi Chen \\ Instrument Technology Research Center, National Applied Research Laboratories, 20 R\&D Road VI, \\ Hsinchu Science Park, Hsinchu 300, Taiwan
}

\begin{abstract}
Automated optical inspection (AOI) has shown its powerful application in many industries. Since little suitable lens options can be found. An optical design is presented to meet the requirements. Nine components is designed, where 2 aspheric ones included. It was found that the image plane size is $62 \mathrm{~mm}$, magnification from 0.025 to 0.14 , and MTF no smaller than $70 \%$ at $47 \mathrm{lp} / \mathrm{mm}$ and $30 \%$ at $142 \mathrm{lp} / \mathrm{mm}$. The focal length was designed around $60 \mathrm{~mm}$.
\end{abstract}

Keywords: lens design, automatic optical inspection, field of view, linear sensor

\section{Introduction}

Automated optical inspection (AOI) has been widely applied in industries such as printed circuit board (PCB), liquid crystal display (LCD), et al. these two decays (Taha et al. 2014) . Key components of AOI system are lens, detector and algorithm. Sometimes different angles of viewing, precision stage mechanism or different spectral filters are arranged. There are cases when suitable optical lens are not available regarding that better and better spatial resolution is always desired. New optical design shall be considered as a result.

A group of requirement was raised. Spectral range was selected at visible range. Focal plane of the lens is linear sensor with pixel size of $5 \mu \mathrm{m}$ and pixel number of $12,000(12 \mathrm{k} 5 \mu \mathrm{m})$ or pixel size of $3.5 \mu \mathrm{m}$ and pixel number of $16,000(16 \mathrm{k} 3.5 \mu \mathrm{m})$. The magnification of the lens varies from 0.025 to 0.14 , i.e. for $12 \mathrm{k} 5 \mu \mathrm{m}$ camera object resolution shall be from 35.7 to $200 \mu \mathrm{m}$, and for $16 \mathrm{k} 3.5 \mu \mathrm{m}$ camera, object resolution from 25 to $140 \mu \mathrm{m}$. Modulation transfer function (MTF) shall be no less than $60 \%$ for spatial frequency of $47 \mathrm{lp} / \mathrm{mm}$ and no less than $30 \%$ for $142 \mathrm{lp} / \mathrm{mm}$. Optical distortion shall be no larger than $0.1 \%$ at all fields. Focal length was set around 60 $\mathrm{mm}$. Since the requirements were stringent, necessary optical design was performed.

*tmw@itrc.narl.org.tw; phone 8863 537-0871

\section{Optical design}

Since the focal length was defined around $60 \mathrm{~mm}$ and magnification changes. From paraxial theory (Yeh et al, 1995), one can calculate the overall distance, $T$, from object to image as

$T=\left(2-M-\frac{1}{M}\right) f$,

where $M$ is the magnification and $f$ the focal length. We can found that $T$ varied from $557 \mathrm{~mm}$ to $2521.5 \mathrm{~mm}$ when magnification from -0.025 to -0.14 (following the sign convention of Welford 1986) if the focal length is $60 \mathrm{~mm}$. On the other hand, if the focal length did not be specified, zoom lens type optical design can be selected. If we set $T$ fixed as $2500 \mathrm{~mm}$, focal length varies from 59.5 to 269.3 $\mathrm{mm}$.

It was found that optical design that met the requirements can be easily found when focal length was larger than $100 \mathrm{~mm}$, where all the components can be spherical. There was some difficulty when trying to make the focal length around $60 \mathrm{~mm}$. If we tried to meet requirements without fixed focal length constrain, focal length of $105 \mathrm{~mm}$ can found. The resulting optical layout is depicted in Figure 1. The focal plane disk diameter was selected as $62 \mathrm{~mm}$. Eight spherical components were used. Maximum clear aperture was $33 \mathrm{~mm}$. The last three components were selected as floating group during 
magnification variation. As a result, the length of the lens was from 53.9 to $54.5 \mathrm{~mm}$. Back focal length was from 74.3 to $86.3 \mathrm{~mm}$.

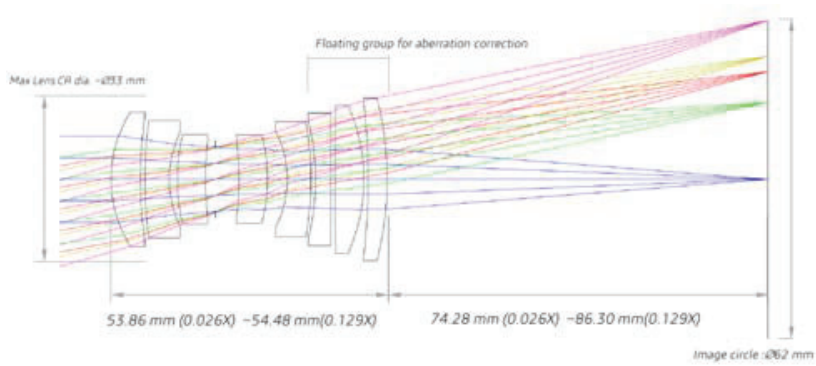

Figure 1. Optical layout for the design result with focal length of $105 \mathrm{~mm}$

To meet the requirements, focal length shall be around $60 \mathrm{~mm}$. The design result is illustrated in Figure 2. Nine components can be found with 2 plastic and 7 glass ones. Four components were arranged in front of the stop while the other five after stop. The last five components were set as floating group when magnification varies. Two aspheric components were designed to meet the stringent requirements such as focal length, MTF and distortion. Maximum lens clear aperture was $86 \mathrm{~mm}$.

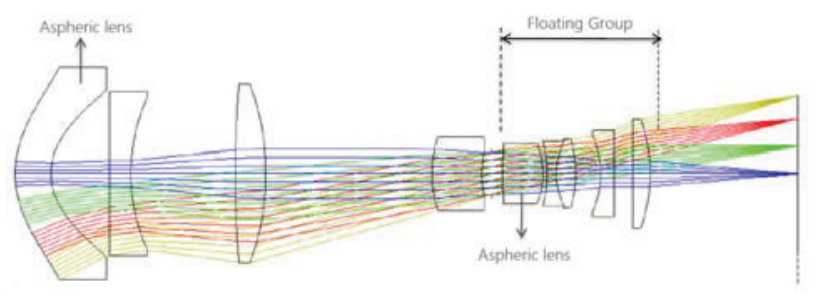

Figure 2. Optical layout for the design result with focal length of $60 \mathrm{~mm}$

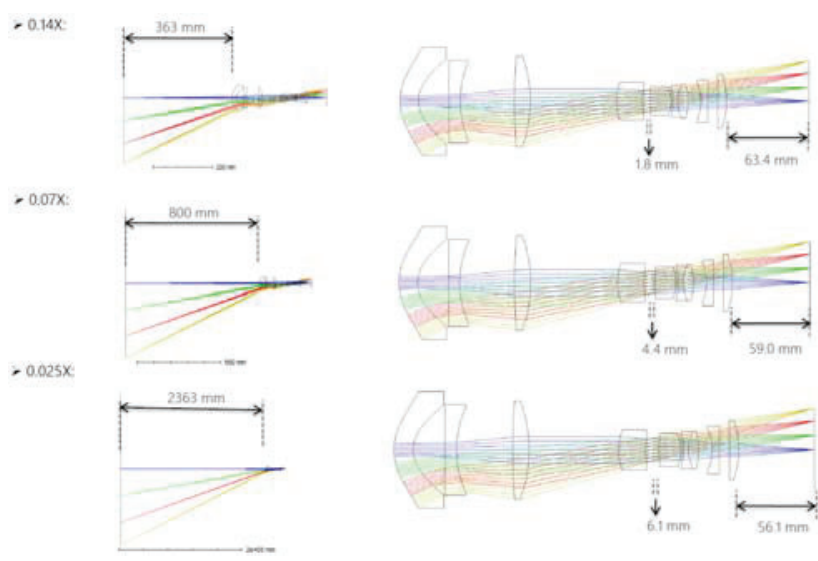

Figure 3. Optical layout for different magnification
In Figure 2, first component was highly asphric for large FOV of $54 \mathrm{deg}$. The length of the lens was from 280.3 to $285.8 \mathrm{~mm}$, while back focal length was from 60.7 to 68 $\mathrm{mm}$. For different magnification, optical layout can be found in Figure 3. Position variation was shown for each case. MTF curves for magnification of 0.025 are shown in Figure 4. For other magnification factors, MTF are slightly different but all larger than $30 \%$ at $142 \mathrm{lp} / \mathrm{mm}$. Distortion was found to be less than $0.1 \%$ for all magnification factors.

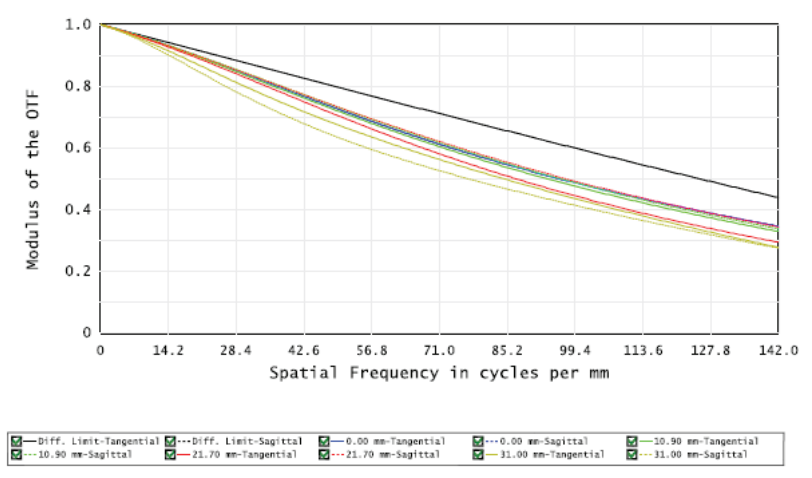

Figure 4. MTF plot for magnification of 0.025

\section{Conclusion}

An AOI lens has been designed for linear sensors with pixel size of $5 \mu \mathrm{m}$ and pixel number of 12,000 or pixel size of $3.5 \mu \mathrm{m}$ and pixel number of 16,000 . The field of view reaches 54 degrees. Magnification of the lens varies from 0.025 to 0.14 . Effective focal length is $60 \mathrm{~mm}$. MTF of optical design meets requirement and reaches $70 \%$ at spatial frequency of $47 \mathrm{lp} / \mathrm{mm}$ and $30 \%$ at $142 \mathrm{lp} / \mathrm{mm}$. The distortion is controlled under $0.1 \%$.

\section{References}

Taha, E. M., Emary, E. and Moustafa, K. 2014. Automatic Optical Inspection for PCB Manufacturing: a Survey, International Journal of Scientific \& Engineering Research 5(7):1095-1102.

Welford, W. T. 1986. Aberrations of Optical Systems. Bristol and Boston: Adam Hilger.

Yeh, M. S., Shiue, S. G. aud Lu, M. H. 1995, Two-opticalcomponentmethod for designing zoom system," Optical Engineering, 34(6): 1826-1834. 


\title{
Characterization of Erbium Doped Phosphate Glasses by Terahertz Time
}

\section{Domain Spectroscopy}

\author{
Yushi Chu ${ }^{\mathrm{a}, \mathrm{b}}$, Shaghik Atakaramians ${ }^{\mathrm{b}}$, Runan Zhang ${ }^{\mathrm{a}}$, Desheng Fan ${ }^{\mathrm{b}}$, Gui Xiao ${ }^{\mathrm{b}}$, Xinghu Fu ${ }^{\mathrm{b}, \mathrm{c}}$, Shuen \\ Wei $^{\mathrm{b}}$, Bowen Zhang ${ }^{\mathrm{b}}$, Yuan Tian ${ }^{\mathrm{b}}$, Zhanyu Ma ${ }^{\mathrm{a}}$, Quan Chai ${ }^{\mathrm{a}}$, Jing Ren ${ }^{*}$, , Yanhua Luo ${ }^{\mathrm{b}}$, Jianzhong \\ Zhang ${ }^{* a}$, Gang-Ding Peng ${ }^{\mathrm{b}}$ \\ ${ }^{a}$ Key Lab of In-Fiber Integrated Optics of Ministry of Education, School of Science, Harbin \\ Engineering University, Harbin, 150001, China \\ ${ }^{b}$ Photonics and Optical Communications, School of Electrical Engineering and Telecommunications, \\ University of New South Wales, Sydney 2052, NSW, Australia \\ 'School of Information Science and Engineering, Yanshan University, Qinhuangdao, 066004, China
}

\begin{abstract}
Terahertz time domain transmission spectroscopy (THz-TDS), simultaneous recording amplitude and phase information with short pulses of terahertz radiation, stand out as an excellent method for material characterization and analysis. In this work, THz-TDS was used to characterize erbium doped phosphate glasses with different compositions. Absorption coefficients, refractive indices and related material parameters were calculated based on these $\mathrm{THz}$ signals, which changed with glass compositions. Furthermore, the relationship between these parameters and optical properties, such as luminescence intensities at $1532 \mathrm{~nm}$ and related fluorescence lifetimes under $980 \mathrm{~nm}$ excitation was demonstrated for the first time to our best knowledge. Results show THz-TDS provides a useful insight into active glass characterization.
\end{abstract}

Keywords: terahertz ray, time domain spectroscopy, phosphate glass, erbium doped material, near infrared emission.

\section{Introduction}

Glass is the most significant high-performance materials used for numerous technologies - including scientific research, industrial and social applications, for example, the luminescent glasses obtained by doping. The past decades have witnessed a significant benefit from these kinds of luminescence glasses in various applications such as fiber-optic communication, laser and light emitting diode (LED) lighting, mainly owing to the outstanding performance of active dopants [1]. A series of photoluminescence ions are inlaid into the host matrix in these applications. The spectroscopic features of gain materials are closely associated with their chemical state and the surrounding ligand field. This provides the possibility to improve the luminescence characteristics of active ions by changing the host matrix identified by Raman spectra, X-ray diffraction, nuclear magnetic resonance [2,3]. Recently THz-TDS has been employed to characterize a series of silicate glasses [4,5]. THz-TDS gives a direct measurement of the field amplitude and phase and enables the calculation of the absorption coefficients and refractive indices with single measurement. But THz-TDS is less used to study active glasses.

In this paper, THz-TDS is used to characterize erbium doped phosphate glasses for the first time to our best knowledge. Material parameters derived from $\mathrm{THz}$ signals were strongly dependent on the glass compositions. The relationships between these parameters and optical properties, such as luminescence intensities at $1532 \mathrm{~nm}$ and related fluorescence lifetimes under $980 \mathrm{~nm}$ excitation, were further discussed and analyzed.

\section{Preparation of doped phosphate glasses}

A set of ytterbium and erbium doped phosphate glass samples, in varying compositions $\mathrm{P}_{2} \mathrm{O}_{5}-\mathrm{X}_{2} \mathrm{O}_{3}-\mathrm{YO}$ with $\mathrm{X}=\mathrm{B}, \mathrm{Al}, \mathrm{Ga}$ and $\mathrm{Y}=\mathrm{Zn}, \mathrm{Mg}, \mathrm{Ca}$, are listed in Table 1. They were prepared by the traditional melting-quenching method [2]. The raw materials were weighed to $\sim 50 \mathrm{~g}$, mixed in an agate mortar for at least 15 min, stored in an alumina crucible and put into a $500{ }^{\circ} \mathrm{C}$ electric furnace for $30 \mathrm{~min}$. Then the samples were melted at $1500{ }^{\circ} \mathrm{C}$ for 60 min. All the obtained glasses were annealed around $\mathrm{T}_{\mathrm{g}}$ for 180 min. Finally, the glasses were cut and polished 
carefully to meet the requirements for optical measurements.

Table 1. Compositions of doped phosphate glass samples (wt $\%$ )

\begin{tabular}{|c|c|c|c|c|c|c|c|c|c|}
\hline Sample & $\mathrm{P}_{2} \mathrm{O}_{5}$ & $\mathrm{~B}_{2} \mathrm{O}_{3}$ & $\mathrm{Al}_{2} \mathrm{O}_{3}$ & $\mathrm{Ga}_{2} \mathrm{O}_{3}$ & $\mathrm{ZnO}$ & $\mathrm{MgO}$ & $\mathrm{CaO}$ & $\mathrm{Yb}_{2} \mathrm{O}_{3}$ & $\mathrm{Er}_{2} \mathrm{O}_{3}$ \\
\hline PGaZn & 74.1 & & & 11.1 & 14.8 & & & 1.2 & 0.6 \\
\hline PAlZn & 74.1 & & 11.1 & & 14.8 & & & 1.2 & 0.6 \\
\hline PBZn & 74.1 & 11.1 & & & 14.8 & & & 1.2 & 0.6 \\
\hline PBMg & 74.1 & 11.1 & & & & 14.8 & & 1.2 & 0.6 \\
\hline PBCa & 74.1 & 11.1 & & & & & 14.8 & 1.2 & 0.6 \\
\hline
\end{tabular}

\section{Terahertz characterization: results and discussions}

Terahertz measurements were conducted using a Menlo Systems GmbH TeraSmart Terahertz Time Domain Spectrometer. The amplitude and phase of the THz signal as a function of frequency were obtained from the measured time-domain data of $\mathrm{THz}$ electric field using the fast Fourier transform (FFT function was used directly in the Matlab). Then the absorption coefficients $(\alpha(v))$ and refractive indices $(n(v))$ of the samples were calculated using following equations:

$n(v)=1+\frac{c}{2 \pi v d}\left[\varphi_{\text {Sam }}(v)-\varphi_{R e f}(v)\right]$

$\alpha(v)=-\frac{2}{d} \ln \left[r(v) \frac{(n(v)+1)^{2}}{4 n(v)}\right]$

$r(v)=\frac{E_{S a m}(v)}{E_{R e f}(v)}$

where $E(v)$ and $\varphi(v)$ are the amplitude and phase of $\mathrm{THz}$ field at frequency $v, c$ is the velocity of light, $d$ is the thickness of samples [6].

Figs. 1. (a) and (b) show the absorption coefficients and refractive indices, respectively. Savitzky-Golay filter has been used to smooth the data in the trusted measurement range $(0.3-1.7 \mathrm{THz})$. For the absorption coefficients, there is no significant difference among glass samples with a fixed $\mathrm{P}_{2} \mathrm{O}_{5}$ to $\mathrm{ZnO}$ ratio (green, red and black curves) while $\mathrm{B}_{2} \mathrm{O}_{3}$ being replaced by $\mathrm{Al}_{2} \mathrm{O}_{3}$ or $\mathrm{Ga}_{2} \mathrm{O}_{3}$. However, when $\mathrm{ZnO}$ is replaced by $\mathrm{MgO}$ or $\mathrm{CaO}$ with a fixed $\mathrm{P}_{2} \mathrm{O}_{5}$ to $\mathrm{B}_{2} \mathrm{O}_{3}$ ratio, the absorption coefficients increase. This can be attribute to the modifier field strength effects [4]. In the system of PBZn, PAlZn and PGaZn, $\mathrm{Zn}^{2+}$ ions mainly play the role of glass network intermediate and certain part of $\mathrm{Zn}^{2+}$ ions can be considered as the glassy network modifiers. However, in the PBMg and PBCa systems, $\mathrm{Mg}^{2+}$ and $\mathrm{Ca}^{2+}$ not only are the network modifiers but also have higher alkaline earth cations in the glasses. It is also observed that the absorption losses increase by replacing the modifier from $\mathrm{Ca}^{2+}$ to $\mathrm{Mg}^{2+}$. Similar phenomenon is observed in Fig. 1 (b), $\mathrm{PBCa}$ and $\mathrm{PBMg}$ have relatively higher refractive indices as compared to PBZn, PAlZn and PGaZn, however, the difference is not obvious as absorption coefficients.

(a)

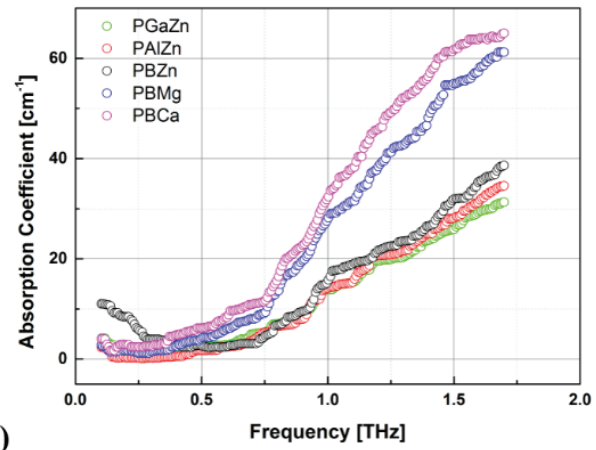

(b)

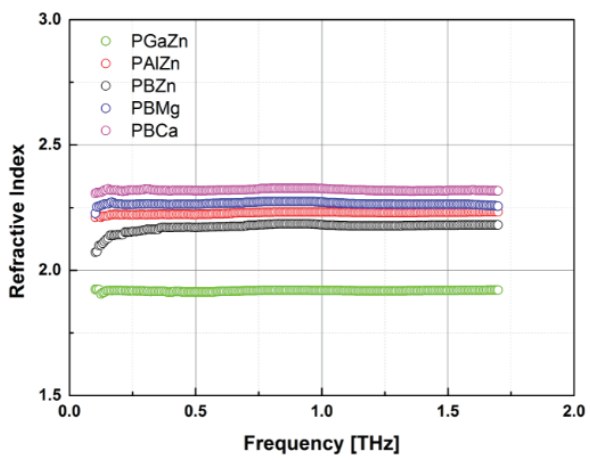

Fig. 1. (a) Absorption coefficients and (b) refractive indices of the glasses studied.

Quantitatively, the effect of glass composition on $\mathrm{THz}$ signals can be understood according to the following equation:

$n(v) \alpha(v)=K(h v)^{\beta}$ 
where $h$ is Plank constant, $\beta$ is an exponent coefficient equaling approximately to two in glassy materials $[4,7]$, and $K$ is a factor which increases with the density of charge fluctuations in the material and the refractive index. (a)

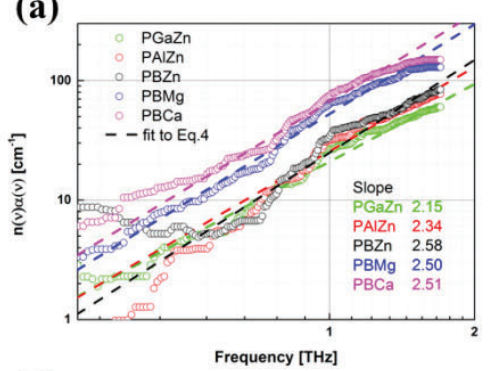

(d)

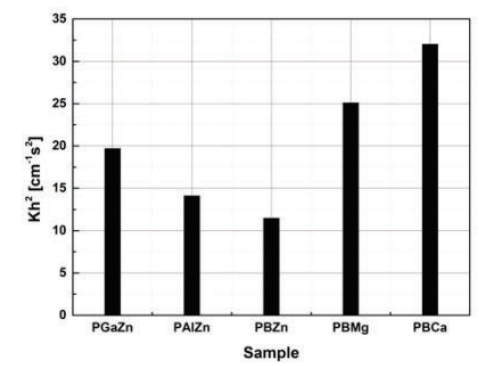

(b)

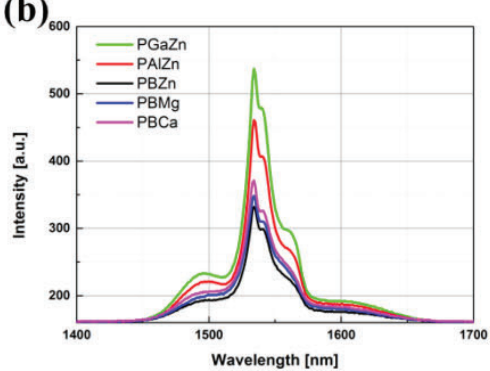

(e)

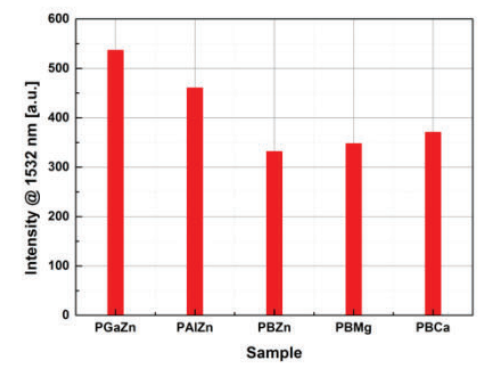

(c)

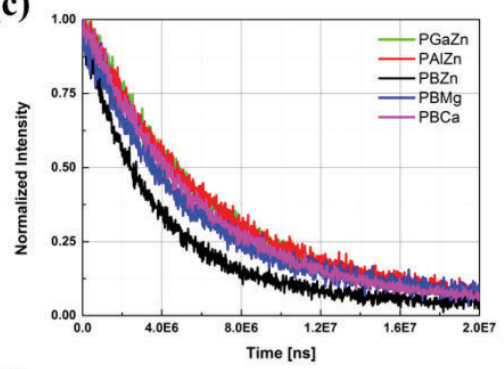

(f)

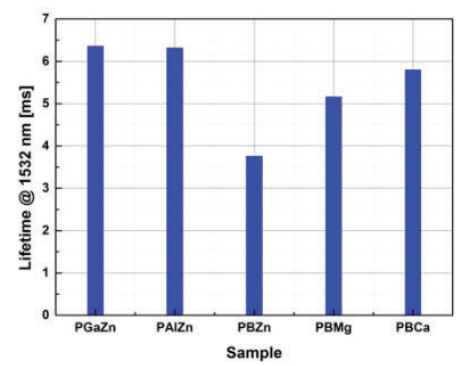

Fig. 2. (a) Frequency dependence of the $n(v) \alpha(v)$ of glasses studied, together with a fit to Eq. (4) (dashed line). (b) Emission spectra.

(c) Decay curves at $1532 \mathrm{~nm}$. (d) Product $\mathrm{Kh}^{2}$ of each sample. (e) Emission intensity and (f) fluorescence lifetime at $1532 \mathrm{~nm}$. All samples are excited by $980 \mathrm{~nm}$ laser.

Fig. 2 (a) shows the $n(v) \alpha(v)$ against frequency on a $\log$-log scale with the values of $\beta$ (slope) listed. Linear fitting has been used ( 0.4 to $1.7 \mathrm{THz}$ ) to obtain material parameters $\beta$ and $\mathrm{K}$, where the $\mathrm{R}$-squares of fitting are $0.978,0.964,0.978,0.979,0.986$ for PGaZn, PAlZn, PBZn, PBMg and PBCa, respectively. The calculated $\mathrm{Kh}^{2}$ values (instead of $\mathrm{K}$ for easier comparisons) based on Eq. (4) are demonstrated in Fig. 2 (d). Both $\beta$ and $\mathrm{K}$ are dependent on the glass composition. The minimum value of $\mathrm{Kh}^{2}$ appears in the PBZn sample, and replacing either $\mathrm{B}_{2} \mathrm{O}_{3}$ or $\mathrm{ZnO}$ will lead to an increase in $\mathrm{Kh}^{2}$.

The change in glass network can also be reflected by emission properties of erbium ions as they will affect by the surrounding ligand field. Fig. 2 (b) and (e) show the $\mathrm{Er}^{3+}{ }^{4} \mathrm{I}_{13 / 2} \rightarrow{ }^{4} \mathrm{I}_{15 / 2}$ emission spectra and luminescence intensity of $1532 \mathrm{~nm}$ under $980 \mathrm{~nm}$ pumping, respectively. The decay curves at $1532 \mathrm{~nm}$ were measured and lifetime were calculated with exponential decay fitting, shown in Fig. 2 (c) and (f). The emission intensity and fluorescence lifetime have similar trends to that of $\mathrm{Kh}^{2}$. The PBZn glass has the minimum value in all three measurements, and substituting $\mathrm{ZnO}$ by $\mathrm{MgO}$ or $\mathrm{CaO}$ and or $\mathrm{B}_{2} \mathrm{O}_{3}$ by $\mathrm{Al}_{2} \mathrm{O}_{3}$ or $\mathrm{Ga}_{2} \mathrm{O}_{3}$ increases $\mathrm{Kh}^{2}$ and fluorescence lifetime and intensity values. Note that the increase is different in each approach, i.e. the presence of $\mathrm{Mg}^{2+}$ and $\mathrm{Ca}^{2+}$ increases $\mathrm{Kh}^{2}$ markedly in $\mathrm{PBZn}, \mathrm{PBMg}$ and $\mathrm{PBCa}$, while the emission intensity and fluorescence lifetime show only moderate variations. Although the presence of $\mathrm{Mg}^{2+}$ and $\mathrm{Ca}^{2+}$ modifies the microscopic polarizability and disorder of the glass network, emission properties are still influence by the phonon energy of glass $[4,8]$. Previous studies have shown that $\mathrm{B}_{2} \mathrm{O}_{3}$ contained glasses mainly consist of $\left[\mathrm{BO}_{3}\right]$ and $\left[\mathrm{BO}_{4}\right]$ with vibrational energies being approximately 1324 $\mathrm{cm}^{-1}$ [2]. Such large phonon energy leads to high nonradiative transition. Whereas the phonon energy of $\mathrm{PAlZn}$ and PGaZn is lower than it in PBZn, PBMg and PBCa, resulting enhanced near infrared emission.

\section{Conclusion}

Erbium doped phosphate glasses with compositions of $\mathrm{P}_{2} \mathrm{O}_{5}-\mathrm{X}_{2} \mathrm{O}_{3}-\mathrm{YO}, \mathrm{X}=\mathrm{B}, \mathrm{Al}, \mathrm{Ga}$; $\mathrm{Y}=\mathrm{Zn}, \mathrm{Mg}, \mathrm{Ca}$, doped with ytterbium and erbium were prepared and characterized by terahertz time domain spectroscopy. The change of $\mathrm{THz}$ 
signals depends on the glass compositions. For the absorption coefficients and refractive indices, they increase as the alkalinity of the glass rises. As for the material parameters $\beta$ and $\mathrm{Kh}^{2}$, they demonstrate similar trends for the emission of $\mathrm{Er}^{3+}$ and fluorescence lifetime at $1532 \mathrm{~nm}$ with subtle differences related to the phonon energy of the glasses. These results demonstrate that THz-TDS has great potential to be employed as a structural probe for active glass characterization.

*zhangiianzhong@hrbeu.edu.cn and ren.jing@hrbeu.edu.cn

\section{Acknowledgements}

This study was supported by the National Natural Science Foundation of China (NSFC) (61775045, 61605030, 51872055), Natural Science Foundation of Heilongjiang Province of China (F2017006), Y. Chu. thanks for the support of China Scholarship Council (CSC No. 201706680054).

\section{Reference}

[1] Chu, Y., Hu, Q., Zhang, Y., et al, "Topological Engineering of Photoluminescence Properties of Bismuthor Erbium-Doped Phosphosilicate Glass of Arbitrary $\mathrm{P}_{2} \mathrm{O}_{5}$ to $\mathrm{SiO}_{2}$ Ratio," Advanced Optical Materials 6(13), 1800024, (2018).

[2] Chu, Y., Ren, J., Zhang, J., et al, “ $\mathrm{Ce}^{3+} / \mathrm{Yb}^{3+} / \mathrm{Er}^{3+}$ triply doped bismuth borosilicate glass: a potential fiber material for broadband near-infrared fiber amplifiers," Scientific Reports 6, 33865 (2016).

[3] Uesbeck, T., Eckert, H., Youngman, R., et al, "The Structure of borophosphosilicate pure network former glasses studied by multinuclear NMR spectroscopy," The Journal of Physical Chemistry C 121(3), 1838-1850 (2017). [4] Naftaly, M. and Miles, R. E., "Terahertz Time-Domain Spectroscopy for Material Characterization," Proceeding of IEEE 95, 1658-1665 (2007).

[5] Withayachumnankul, W., Png, G. M., Yin, X., et al, “Tray sensing and imaging," Proceedings of the IEEE 95, 1528-1558 (2007).

[6] Jepsen, P. U., and Fischer, B. M., "Dynamic range in terahertz time-domain transmission and reflection spectroscopy," Optics Letters 30(1), 29-31 (2005).

[7] Naftaly, M. and Miles, R. E., "Terahertz time-domain spectroscopy of silicate glasses and the relationship to material properties," Journal of Applied Physics 102(4), 043517 (2007).

[8] Chu, Y., Ren, J., Zhang, J., et al, "Effects of melting temperature and composition on spectroscopic properties of $\mathrm{Er}^{3+}$-doped bismuth glasses," Optical Materials Express 6(1), 279-287 (2016). 


\title{
Performance Analysis of Structured Light Elements with Various
}

\section{Diffraction Patterns}

\author{
Rou-Jhen Chen ${ }^{\mathrm{a}}$, Yu-Hsuan Lin ${ }^{\mathrm{a}}$, Hsin-Yi Tsai ${ }^{\mathrm{a}}$, Kuo-Cheng Huang ${ }^{\mathrm{a}}$, Chun-Han Chou ${ }^{\mathrm{a} *}$ \\ a. National Applied Research Laboratories, Instrument Technology Research Center, 20, R\&D Rd. VI, Hsinchu Science Park, Hsinchu City, Taiwan, \\ R.O.C. 30076
}

bonochou@narlabs.org.tw

\begin{abstract}
In the studying, we analysis the diffraction pattern noise that was design by iterative Fourier transform algorithm. 3D scanning technology widely used in our life. Structure light was a popular technology that used in the 3D scanning. The projection pattern of structure light directly affected the system resolution. Therefore, we design the two types of diffraction pattern by iterative Fourier transform algorithm and compare the imaging quality. From the simulation result, we found the circular pattern which Signal to Noise Ratio was 1.5 times to the rectangular type and the projection pattern didn't directly affect conversion efficiency and stray light. In the future, we could simulate the more patterns and complex patterns to find the best projection pattern for structured light.
\end{abstract}

Keywords: diffraction pattern, iterative Fourier transform algorithm, structure light, 3D scanning

\section{Introduction}

Currently, 3D scanning technology widely used in our life. The technology flourished from the 2010. The most people well known was Kinect. The system was published by Microsoft. It could measure the depth information and instant computing the full color model. The tech not only used in the entertainment, but also applied to medical, 3D imaging construct and health care etc. [1] The 3D scanning technology could be classified to active type and passive type. The most popular 3D scanning technology was list on the below: (reference Figure. 1)

- Stereo Vision (passive type): The tech used the parallax principle, so the system usually needed two or more than two imaging from the different angle. The 3D information could be obtained by each relative positions of objects in these plane screens. [2]

- Structured Light (active type): The tech usually composed of the IR light source and IR camera. The light source projected a certain pattern on the object and the IR camera will capture the reflection figure. The $3 \mathrm{D}$ information could be obtained by the difference between the ideal pattern and reflection pattern. [3, 4]
- Time of Flight (active type): The major elements of the tech was the same with structure light, but it used the different working principle. The 3D information could be obtained by each flight time of light source. [5]

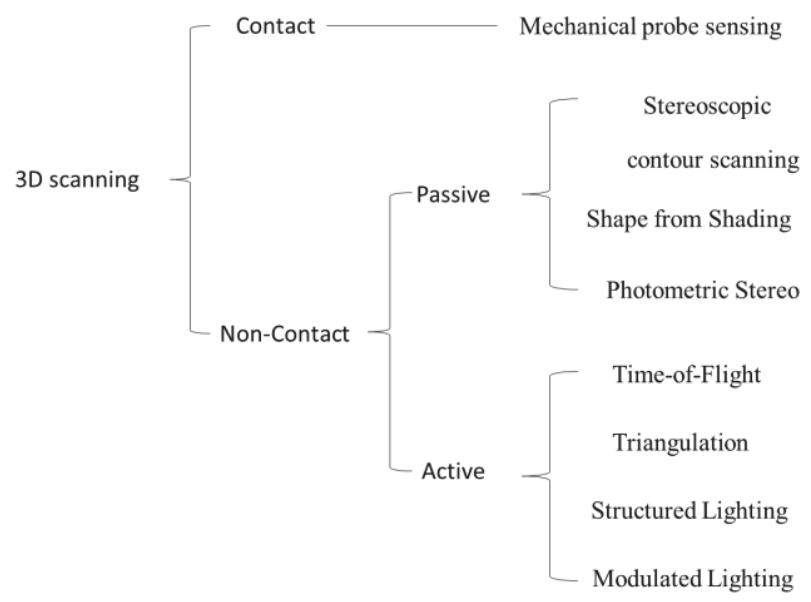

Figure. 1 3D scanning technology classification

Most of the 3D scanning products was active type. In the above popular scanning techs, the structured light technology was the only one could analysis nanometer depth information, but it still had some disadvantage of sensor calibration, environment light effects and long operation time. Therefore, people present the different 
method to overcome these problems. El-laithy team presented an algorithm to reduce the environment light effects [6]. Wiedemann team change the object distance and camera exposure time to decrease the calibration time [7]. Hall-Holt team presented a special pattern to decrease the system operation time [8]. Zhang team used the color pattern and phase shift principle to increase the depth information resolution $[9,10]$. In these prior art, lot of people presented the different meth to reduce the noise and operation time. In the paper, we would like to use the Iterative Fourier Transform Algorithm(IFTA) to design the different patterns grating and compare the noise which applied in the structured light system.

\section{Diffraction element design principle}

In the paper, we used IFTA to design the three grating with the different patterns. IFTA principle was suppose a random phase and through numerical iterative processing to get the result. The calculation process showed on the below: (reference Figure. 2)

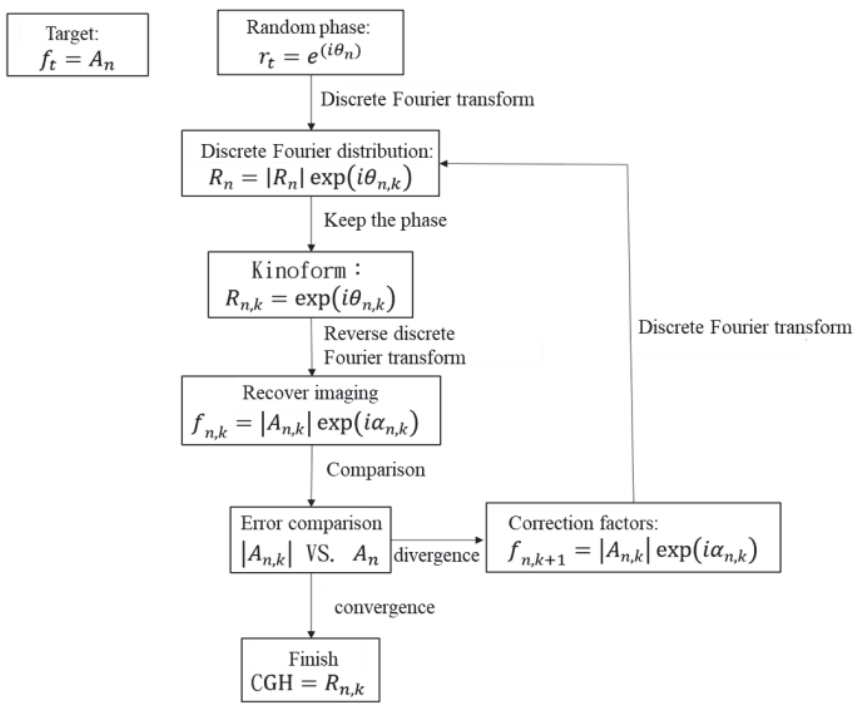

Figure. 2. IFTA diffraction grating creation process

In the experiment, we used the light source with 532 $\mathrm{nm}$ wavelength. The simulation focal length on the $100 \mathrm{~mm}$. The grating was a four level diffraction element and each pitch was $3.04 \mathrm{um}$. Its size was $10 * 10 \mathrm{~mm}$. The simulation structure reference Figure. 3.

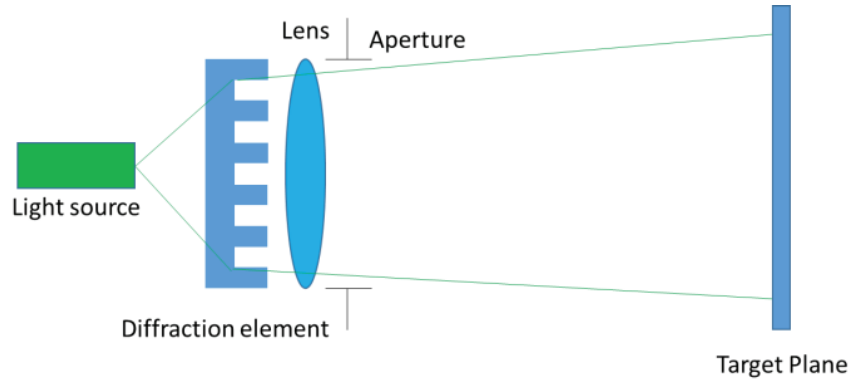

Figure. 3. Experiment diagram

\section{Diffraction pattern simulation results}

In the paper, we used IFTA to design the two diffraction elements which could project the diffraction patterns. The first projection pattern was $5 * 5$ circular matrix. Each spot diameter was $2 \mathrm{~mm}$ and the pitch was $2 \mathrm{~mm}$. (reference Figure. 4) The second projection pattern was $5 * 5$ rectangular matrix. Each spot was $2 \mathrm{~mm}$ and the pitch was $2 \mathrm{~mm}$. (reference Figure. 5)

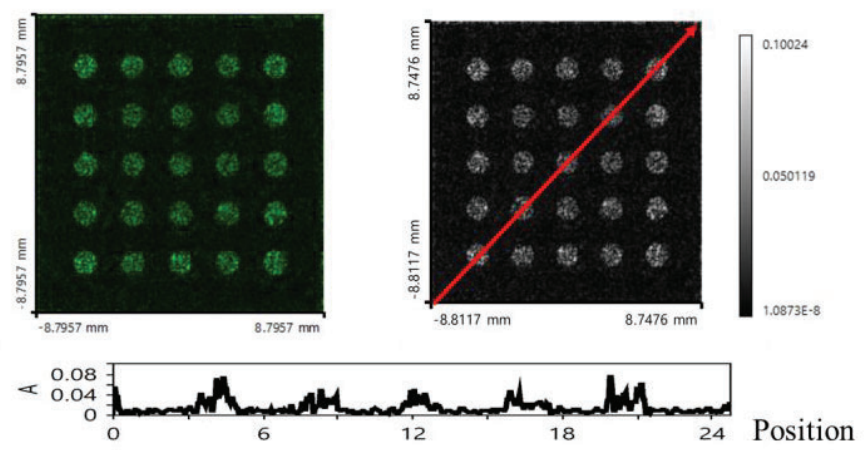

Figure. 4. Circular pattern $5 * 5$ matrix

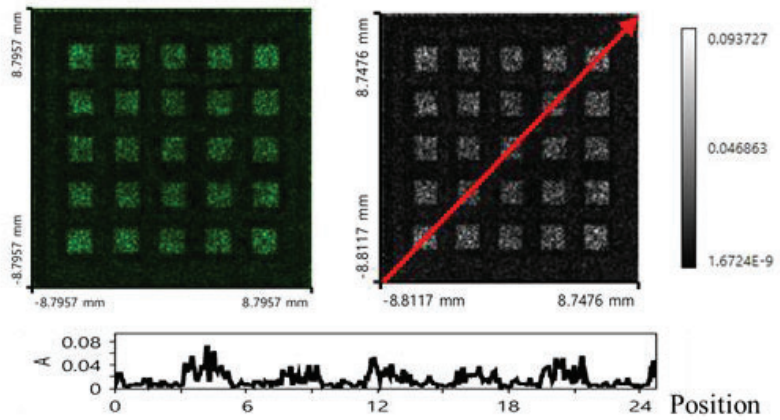

Figure. 5. Rectangular pattern $5 * 5$ matrix

From the simulation result, we compared the results of two type. Signal to Noise Ratio of circular type was 
rectangular type 1.5 times. The two type results of conversion efficiency and stray light was almost the same. (reference Table. 1.)

Table. 1. The two type simulation results

\begin{tabular}{|c|c|c|c|}
\hline Items & $\begin{array}{c}\text { Conversion } \\
\text { Efficiency (\%) }\end{array}$ & $\begin{array}{c}\text { Signal-to-Noise } \\
\text { Ratio(dB) }\end{array}$ & Stray Light (\%) \\
\hline Circular type & $49 \%$ & $20 \mathrm{~dB}$ & $60 \%$ \\
\hline Rectangular type & $51 \%$ & $13 \mathrm{~dB}$ & $61 \%$ \\
\hline
\end{tabular}

Through the simulation, we found the pattern that would directly affect the SNR. The pattern didn't directly affect the conversion efficiency and stray light. The simple projection pattern was more suitable for the structure light system.

\section{Conclusion}

In the studying, we used IFTA to design two types diffraction element. From the simulation results, we found the simpler pattern which Signal to Noise Ratio was 1.5 times to the rectangular type and the projection pattern didn't directly affect conversion efficiency and stray light. In the future, we could simulate the more patterns and complex patterns to find the best projection pattern for structured light.

\section{References}

[1]. Sarbolandi, Hamed, Damien Lefloch, and Andreas Kolb. "Kinect range sensing: Structured-light versus Time-of-Flight Kinect." Computer vision and image understanding 139: 1-20. (2015)

[2]. Tippetts, Beau, et al. "Review of stereo vision algorithms and their suitability for resource-limited systems." Journal of Real-Time Image Processing 11.1: 5-25. (2016)

[3]. Rubinsztein-Dunlop, Halina, et al. "Roadmap on structured light." Journal of Optics 19.1: 013001. (2016)

[4]. Litchinitser, Natalia M., et al. "Structured light-matter interactions in engineered optical media (Conference Presentation)." High Contrast Metastructures VI. Vol. 10113. International Society for Optics and Photonics, 2017.

[5]. Beck, Scarlet, et al. "The impact II, a very high resolution quadrupole time-of-flight instrument for deep shotgun proteomics." Molecular \& Cellular Proteomics: mcp-M114. (2015)
[6]. El-laithy, Riyad A., Jidong Huang, and Michael Yeh. "Study on the use of Microsoft Kinect for robotics applications." Position Location and Navigation Symposium (PLANS), 2012 IEEE/ION. IEEE, 2012.

[7]. Wiedemann, Matthias, et al. "Analysis and characterization of the PMD camera for application in mobile robotics." IFAC Proceedings Volumes 41.2: 13689-13694. (2008)

[8]. Hall-Holt, Olaf, and Szymon Rusinkiewicz. "Stripe boundary codes for real-time structured-light range scanning of moving objects." Computer Vision, 2001. ICCV 2001. Proceedings. Eighth IEEE International Conference on. Vol. 2. IEEE, 2001.

[9]. Zhang, Song, and Peisen Huang. "High-resolution, real-time 3D shape acquisition." null. IEEE, 2004.

[10]. Zhang, Song. "Recent progresses on real-time 3D shape measurement using digital fringe projection techniques." Optics and lasers in engineering 48.2: 149-158. (2010) 


\title{
Research on measurement method of coincidence degree for
}

\author{
remote micro-objects based on parallel light imaging
}

${ }^{1,2}$ Wei han, ${ }^{1,2}$ Min Huang, ${ }^{1,2}$ Qisheng Cai, ${ }^{1,2}$ Xiangning Lu

1.Key Laboratory of Computational Optical Imaging Technology, Chinese Academy of Sciences,2. Academy of Opto-Electronics, Chinese Academy of Sciences

\section{Abstract}

A method of coincidence degree for remote micro-objects based on parallel light imaging is proposed, which solve the problem that the existing technology can not measure the space position of micro-objects at a long distance.

\section{Introduction}

The long-distance measurement of the spatial position of micro-objects that the geometric size is similar to the wavelength is limited by the optical physical characteristics, the accuracy of equipment, and can not meet the accuracy requirements, even can not be measured. Especially for the measurement of the spatial position relationship of multiple micro-objects in long-distance, whether using full-wavelength beam or laser beam to measure, it will be affected by interference and diffraction. In this paper, the coincidence measurement of two micro-slits (tens of microns) with long distances (meters) is studied.

\section{Basic principle of measurement}

Considering the actual situation of the measurement task, the measurement principle as shown in Figure 2 is adopted: white light source 1 emits stable white light, after homogenizing plate 2, homogenizing light 3 irradiates on the first tantalum plate 4 . The tantalum plate is located at the focal point of parallel light shaping device 5 . The parallel light shaping device has a fixed focal length $f(6)$, and then is coupled to another parallel light shaping device 8 through parallel light 7 . This device has the same parameters and technical specifications with the former parallel light shaping device. The second tantalum sheet 10 is placed at the focus of the second parallel light shaping device 8. At this time, the equal-scale real image of the first Tantalum Sheet 4 is inverted on the surface of the second tantalum sheet 10. The position of the inverted real image and the second tantalum sheet is measured by using the detection/observation measuring device 12 with focal length $\mathrm{fO}(11)$ (this project is a reading microscope). Finally, the measurement results of projection coincidence degree are obtained. 


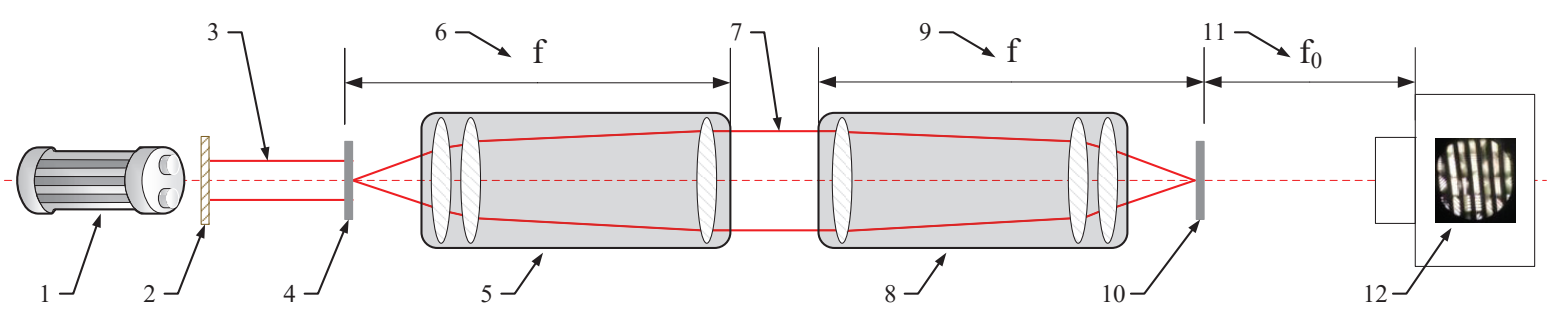

Fig. 1 Principle of coincidence measurement

\section{Measurement process and result}

3.1 Determination of sampling points

During the sampling process, in order to ensure that the sampled sample can universally and reasonably represent the projection size of the micro-slit in the whole $20{ }^{*} 20 \mathrm{~mm}$ range, and have a good response system setting accuracy, sampling points are selected according to the figure 2. That is to say, it can cover the range of edges and corners, and at the same time reflect the changing trend of projection size of horizontal and vertical slits, and basically determine the accuracy distribution of the alignment results.

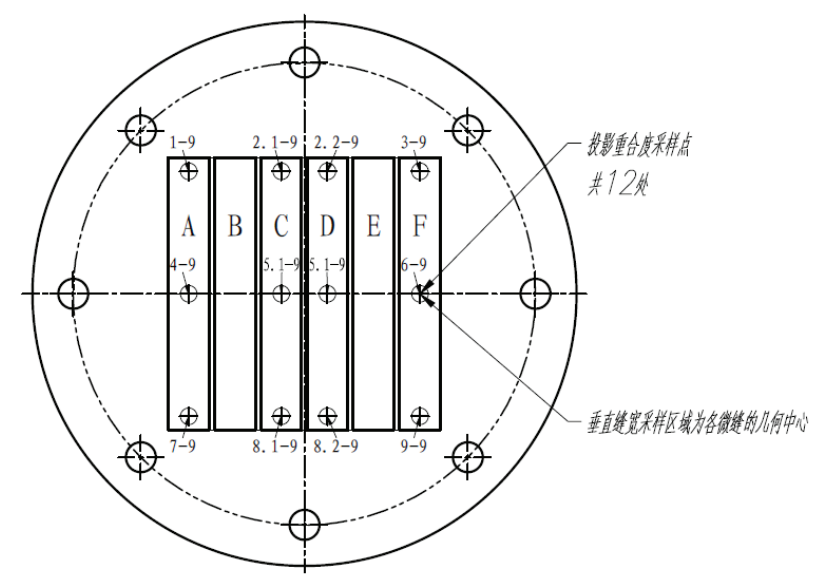

Figure 2 Sampling schematic diagram of Ta slit coincidence data

\subsection{Focus calibration}

Using standard devices such as bolo plate and reading microscope to finish focus calibration.

\subsection{Coaxial calibration}

Using linear interval and knife-edge method of photoelectric conversion of photodetectors to finish coaxial calibration.

\subsection{Rusult}

The average slot width of tantalum sheet is 146.37 micron, and the projection slot width of the collimator is 141.19 micron after fine-tuning. The average coincidence rate is $96.461 \%$. 


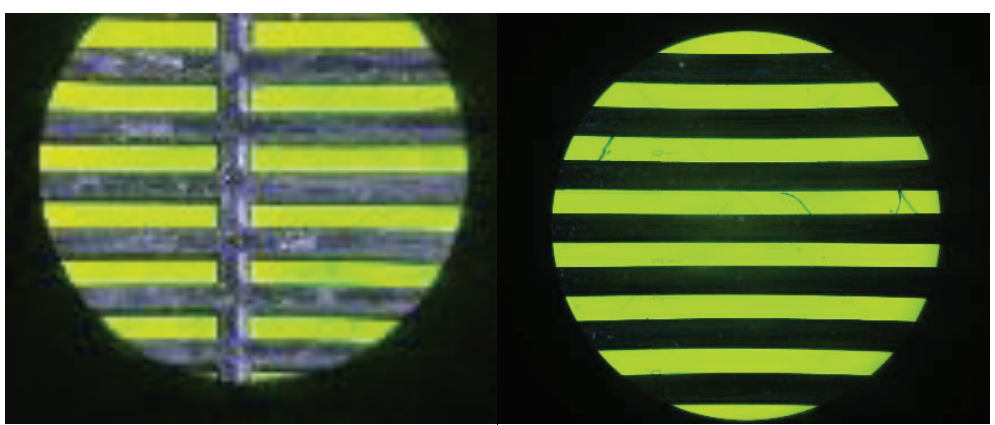

Fig. 3 Impression drawing of alignment

\section{Conclusions}

The measurement method of coincidence degree for remote micro-objects based on parallel light imaging has the following advantages:

(1) Avoiding interference and diffraction phenomena in remote micro-object detection by using parallel light imaging method.

(2) Using the transfer process of optical imaging system to eliminate one dimension variable in three-dimensional space problem and improve measurement accuracy. 
准直器镜筒装调精度测量

本测量方法, 经过与客户协商确定, 采用自行研制方法检测, 具体参照 “2016ZY606009 准直镜筒精测及装配”。对准直器装配体对应位置钽片组的精密装调进行实时测量, 配合完 成准直器装配工作。对实验数据整理如下表 1 、表 2 。

表 1 测量微缝投影垂直宽度数据统计

\begin{tabular}{|c|c|c|c|c|}
\hline $\begin{array}{l}\text { Item } \\
\mathrm{s}\end{array}$ & $\begin{array}{l}\text { Data } \\
(\mu \mathrm{m})\end{array}$ & $\begin{array}{c}\text { Pitch } \\
\text { average }(\mu \mathrm{m} \\
)\end{array}$ & $\begin{array}{l}\text { Hor izontal } \\
\text { average }(\mu \mathrm{m})\end{array}$ & $\begin{array}{c}\text { Vertical } \\
\text { average }(\mu \mathrm{m})\end{array}$ \\
\hline $1-9$ & 122.5 & 122.5 & & \multirow{5}{*}{$\begin{array}{c}142.25 \\
(1-9 、 4-9,7-9)\end{array}$} \\
\hline 2. $1-$ & 136.2 & \multirow{3}{*}{135.625} & \multirow{3}{*}{$\begin{array}{c}127.875 \\
(1-9,2.1-9 、 2.2-9 、 3-9)\end{array}$} & \\
\hline 9 & 5 & & & \\
\hline $\begin{array}{l}2.2- \\
9\end{array}$ & 135 & & & \\
\hline $3-9$ & 125.5 & 125.5 & & \\
\hline $4-9$ & $\begin{array}{c}155.7 \\
5\end{array}$ & 155.75 & & \multirow{5}{*}{$\begin{array}{c}151.167 \\
(2.1-9 、 2.2-9 、 5.1-9 、 5.2-9 、 \\
8.1-9 、 8.2-9)\end{array}$} \\
\hline $\begin{array}{c}\text { 5. } 1- \\
9\end{array}$ & 172 & \multirow[b]{2}{*}{166.625} & \multirow{2}{*}{$\begin{array}{c}157.125 \\
(4-9 、 5.1-9 、 5.2-9 、 6-9)\end{array}$} & \\
\hline $\begin{array}{l}\text { 5. } 2- \\
9\end{array}$ & $\begin{array}{c}161.2 \\
5\end{array}$ & & & \\
\hline $6-9$ & 149 & 149 & & \\
\hline $7-9$ & 148.5 & 148.5 & & \\
\hline 8. $1-$ & 151.7 & \multirow{4}{*}{151.25} & \multirow{5}{*}{$\begin{array}{c}138.583 \\
(7-9 、 8.1-9 、 8.2-9 、 9-9)\end{array}$} & \multirow{5}{*}{$\begin{array}{c}130.167 \\
(3-9,6-9,9-9)\end{array}$} \\
\hline 9 & 5 & & & \\
\hline 8. $2-$ & 150.7 & & & \\
\hline 9 & 5 & & & \\
\hline $9-9$ & 116 & 116 & & \\
\hline & Total a & erage & 141 & $94 \mu \mathrm{m}$ \\
\hline
\end{tabular}

表 2 测量微缝投影水平宽度数据统计

\begin{tabular}{cc}
\hline Items & Pitch $(\mathrm{mm})$ \\
\hline A & 3.2 \\
B & 3.1 \\
C & 3.1 \\
D & 3.1 \\
E & 3.1 \\
F & 3.2
\end{tabular}

Proc. of SPIE Vol. 11142 1114201-146 
Total average

Average $=3.13 \mu \mathrm{m}$

$\mathrm{RMS}=0.0022$

采样过程中, 为保证所采样本在整个 $20 \times 20 \mathrm{~mm}$ 微缝范围内能够普适、合理的代表微缝投 影尺寸, 良好的反应系统装调精度, 采样点的选取按照图 1 示意进行采样。即能够覆盖边角 范围, 同时反应水平与垂直方向微缝投影尺寸的变化趋势，基本确定装调结果的精度分布。

一、规定:

$>$ 开机 5 分钟后, 待平行光照明光源功率稳定后, 进行后续测量;

$>$ 开始测量前利用示波器、十字分划板等对测量装置进行校准, 并对测量 装置安装座移动的重复定位性进行校核;

$>$ 将准直器放置在检测工装上, 安装测量装置; 见附图 1、附图 2

$>$ 根据所制定的测量样品选取策略确定微缝的测量位置; 为保证测量结果的普适性, 选取微缝区的 12 处作为测量点;

$>$ 测量过程中关键步骤都需要完成焦点校准, 即, 利用玻罗板等标准器件 配合读数显微镜完成焦点校准; 见附图 3

$>$ 示波器读数规定为采样数据均方差小于限值后方可读数;

$>$ 测量数据及图片规定:

（1）包括测量关键过程照片;

（2） 测量结果视频文件;

（3） 检测数据;

$>$ 参数名称定义:

Pitch：缝宽，有水平、坚直之分;

Horizontal: 水平采样点平均值，用来反映水平统计值的变化趋势;

Vertical: 垂直采样点平均值, 用来反映垂直统计值的变化趋势。

\section{三、测量原理}

考虑到测量任务的实际要求, 采用了如图 2 的测量原理: 白光光源 1 发射稳定白光, 经 过匀光板 2 匀化, 匀化光 3 照射在第一钽片 4 上, 该钽片处于平行光整形器件 5 的焦点处, 该平行光整形器件具有固定焦距 f (6), 而后通过平行光 7 耦合至另一平行光整形器件 8, 该器件与前一平行光整形器件具有相同的参数与技术指标，此时将第二钽片 10 放置在第二 平行光整形器件 8 的焦点处, 此时第一钽片 4 的等比例实像倒置呈在第二钽片 10 的表面, 利用具有焦距 $\mathrm{f}_{0}(11)$ 的探测/观察测量设备 12（本项目为读数显微镜）测量该倒置实像与 第二钽片的位置关系，最终得到投影重合度测量结果。

\section{二、测量流程如下:}


利用塞尺调整准直器筒体、筒顶至可连接状态, 利用筒体机械公差初 步定位两筒顶及其附件的空间位置，如图 2;

$>$ 移除筒体, 安装测量装置, 在筒顶 01 钽片安装位置处安装十字靶片 01 , 在平行光管 01 上安装十字分划板 01 , 利用两十字刻线对齐的原理, 调 整平行光管光轴与钽片安装面至垂直、刻线对齐, 此时钽片机械轴与 平行光管 01 光轴同轴; 需要说明的是, 此时十字靶片 01 与十字分划 板 01 处于与平行光管焦平面平行基本等微距的两个离焦平面上，虽然 离焦, 但对对齐精度影响较小;

$>$ 安装平行光管 02 , 拆除十字靶片 01 , 在平行光管 02 的焦点位置安装 十字分划板 02 , 利用十字刻线对齐的方法将两平行光管校平行;

$>$ 此时, 平行基准以传递至平行光管 02 , 需要对两平行光管进行同轴校 准, 这里利用光电探测器光电转换的线性区间结合刀口法进行同轴校 准, 原理如式 1 2。

$$
I=\frac{\eta e}{h v} P
$$

式 1 光电探测器原理公式

式中, $\eta$ 为量子效率, $P$ 为光功率, $e$ 为电子电荷, $h v$ 代表光子能量, 对于同一标定的探测器系统, 量子效率 $\eta$ 为固定值, 因此可以得出, 式 2 。

$$
I \propto P
$$

设置示波器参数为幅值能量平均采样, 取实时 512 次平均, 设置灵敏 度 $1 \mathrm{mV} \Omega$, 探测器增益设置为 $10 \mathrm{~dB}$, 待示波器示值稳定后读数。水平 错位移动平行光管, 得到系列读数, 即水平位置与示波器光强读数的 关系拟合曲线, 实验中为基本线性曲线, $y$ 轴 (光强) 截距不为零, 即 平行光管参考零点并非实际同轴位置, 则此时反向求解 $y$ 轴截距为零的 $x_{0}$ 值, 调整平行光管水平位移的测微螺杆 $\Delta x=x_{0}$, 此时两平行光管光 轴平行且处于同一竖直平面内; 转动刀口元件 $90^{\circ}$, 再次重复以上步 骤, 使两平行光管光轴平行且处于同一水平平面内; 此后转动刀口 $60^{\circ}$ 多次, 重复以上步骤, 将同轴误差分散至水平、垂直两维方向, 多次 平均系统误差, 达到较好同轴校准效果;

$>$ 撤下其他测量辅助装置, 调整测量装置、准直器主体至测量位置（间 距 $1 \mathrm{~m}$ 左右); 测量期间, 由于读数显微镜口径限制, 需探测区域标定 靶板辅助测量, 利用 $3 \times 3$ 点阵靶板将测量区域人为规定为 9 个圆形区 
域，并在圆形区域内做对齐精调，最终达到整体对齐的效果，如图 8、 9 ;

\section{三、结果分析:}

已知胶结片测量结果为 SAMPLE01, 平均缝宽 $145.14 \mu \mathrm{m}$, SAMPLE02, 平均缝宽 $147.60 \mu \mathrm{m}$ 。

根据报告第一节的测量数据, 总结如下:

1、钽片 $20 \mathrm{~mm} \times 20 \mathrm{~mm}$ 微缝区域内, 精调后对齐投影的平均缝宽为 $141.19 \mu \mathrm{m}$;

2、最大投影缝宽 $172 \mu \mathrm{m}$ ，最小 $116 \mu \mathrm{m}$ ；

3、中心区域投影缝宽度最大, $151 \sim 157 \mu \mathrm{m}$, 越靠近边缘，微缝投影宽度越 小, 调整精度越低;

4、数据采集点为类似 $9 \times 9$ 模式, 横纵分析, 三行采集点数据变化趋势为中 间大，两端小，下端大于上端; 三列采集点数据变化趋势为中间大，两 端小, 左端大于右端, 说明微缝调整效果中间区域最好, 其次为第三象 限 (左下)，再次为第一象限 (右上);

5、水平宽度统计中, 水平宽度一致性良好, 数据波动极小, 平均值接近实 际值, 结果为 $3.13 \mu \mathrm{m}, \mathrm{RMS}=0.0022$ 。

对比胶结片数据, 胶结片平均缝宽 $146.37 \mu \mathrm{m}$, 精调后准直器对应钽片投影㖓宽 $141.19 \mu \mathrm{m}$, 平均重合率为 $96.461 \%$ 。

3. Analysis of results:

The measured results of cemented sheets are SAMPLE01, with an average slit width of 145.14 um, SAMPLE02 and an average slit width of $147.60 \mathrm{um}$.

Based on the measurement data in section I of the report, it is summarized as follows:

1. The average slit width of fine-tuned alignment projection is 141.19 um in the 20 $\mathrm{mm} \times 20 \mathrm{~mm}$ slit area of tantalum sheet.

2. The maximum projection slit width is 172 microns and the minimum is 116 microns.

3. The width of projection slit in the central region is the largest, 151-157 um. The closer to the edge, the smaller the projection slit width and the lower the adjustment accuracy.

4. Data acquisition point is similar to $9 * 9$ mode. The horizontal and vertical analysis shows that the data change trend of three row acquisition points is large in the middle, small at both ends and larger at the lower end than at the upper end. The data change trend of three row acquisition points is large in the middle, small at both ends and larger at the left end than at the right end, indicating that the middle area of micro-slit adjustment effect is the best, followed by the third quadrant (lower left) and the first quadrant (upper right).

5. In horizontal width statistics, the consistency of horizontal width is good, the Proc. of SPIE Vol. 11142 1114201-149 
fluctuation of data is very small, the average value is close to the actual value, the result is 3.13 um, RMS $=0.0022$.

Comparing the data of cementing sheet, the average seam width of cementing sheet is 146.37 micron, and the projection seam width of the collimator is 141.19 micron, the average coincidence rate is $96.461 \%$. 


\title{
Detection of optical vortices using cyclic, rotational and reversal shearing interferometers
}

\author{
K.N.Gavril'eva ${ }^{a}$, A. Mermoul ${ }^{\text {a }}$, A. A. Sevryugin ${ }^{\text {a }}$, E.V.Shubenkova ${ }^{\text {a }}$, \\ M. Touila ${ }^{a}$, I.M.Tursunov ${ }^{a}$, E.A.Efremova ${ }^{b}$, V. Yu. Venediktov ${ }^{a, b}$ \\ ${ }^{a}$ St. Petersburg State Electrotechnical university, St. Petersburg, 197376 Russia \\ ${ }^{\mathrm{b}}$ St. Petersburg State University, Peterhof, St. Petersburg, 198504 Russia \\ vlad.venediktov@mail.ru,vlad_venediktov@yahoo.com
}

\begin{abstract}
In this paper, the determination of the topological charge of the vortex beams by means of shearing interferometry was achieved, for both common and non-common path shearing interferometers, using simple yet effective optical elements. The recording and analysis of interference patterns from different setups was accomplished using: cyclic, rotational and reversal shearing interferometers. The use of cyclic and rotational shearing interferometers resulted in interference patterns with two oppositely oriented forks for both setups. However, with the reversal shearing interferometer, a single forked pattern was obtained and a mathematical approximation was deduced.
\end{abstract}

Keywords - vortex beams, shearing interferometry, topological charge.

\section{Funding:}

\section{Introduction}

Optical vortices with helical wavefronts and phase dependence $e^{i m \theta} ; \mathrm{m}$ being the topological charge and $\theta$ the azimuthal angle in cylindrical coordinates; were recognized to have orbital angular momentum (OAM) only about two decades ago [1]. The important part is that angular momentum is independent of the polarization state. This OAM induced a rise of interest in optical fields possessing screw phase singularities carried by vortex beams due to its wide range of potential applications in various areas, and also its importance from the viewpoint of both fundamental and applied physics.

Algebraically, the set of vortices with integer valued topological charges represents a basis for a space of infinite dimensionality over circular apertures of arbitrary radius [2]. And since the number of these Eigen states is unlimited in theory, there would be a possibility to generate unlimited communication channels using optical vortex beams if they were utilized as an information carrier.

The OAM of a vortex beam is equal to $\mathrm{mh} / 2 \pi$ per photon, where $\mathrm{m}$ is the topological charge and $h$ is planks constant [3-4]. Also, given that the line integral of the phase gradient of the optical vortex around its singular point is nonzero and equals to $2 \pi \mathrm{m}$, the topological charge $\mathrm{m}$ defines how many revolutions the wavefront takes per a wavelength. Hence, by determining this topological charge, full information is provided about the phase profile of the vortex and also its OAM. Figure 1 shows beams with various topological charges from 0 to 3. [5]
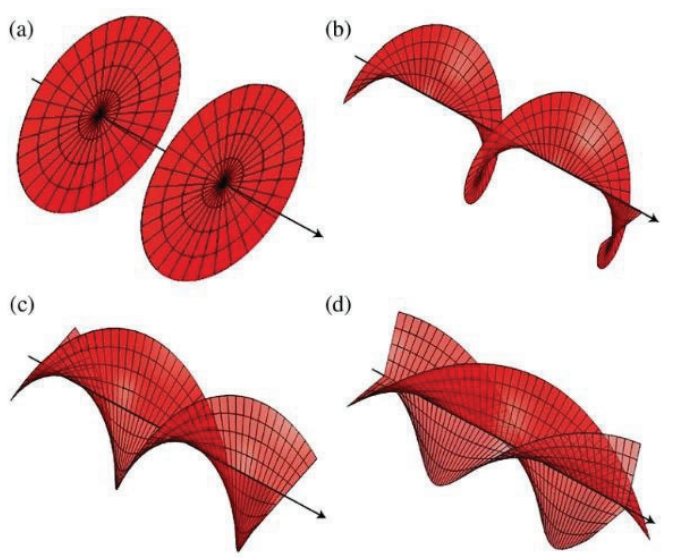

(d)

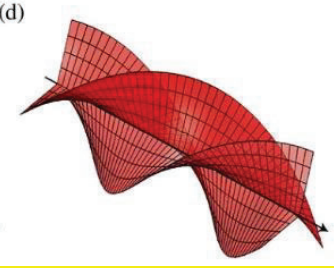

Fig. 1 Beams with topological charges $m=0$ (a); $m=1$ (b); $m=2$ (c); $m=3$ (d).

Like polarized beams carrying spin angular momentum are readily produced by means of a wave-plate to convert linear light to circularly polarized light, a Hermite-Gaussian beam with no angular momentum could be similarly transformed with cylindrical lenses into a Laguerre-Gaussian beam carrying orbital angular momentum, as shown in Figure 2a. Consequently, numerically computed holograms have been the most common 
method of creating helical beams, as they can generate any Laguerre-Gaussian mode from the any initial beam (Figure 2b). [6]
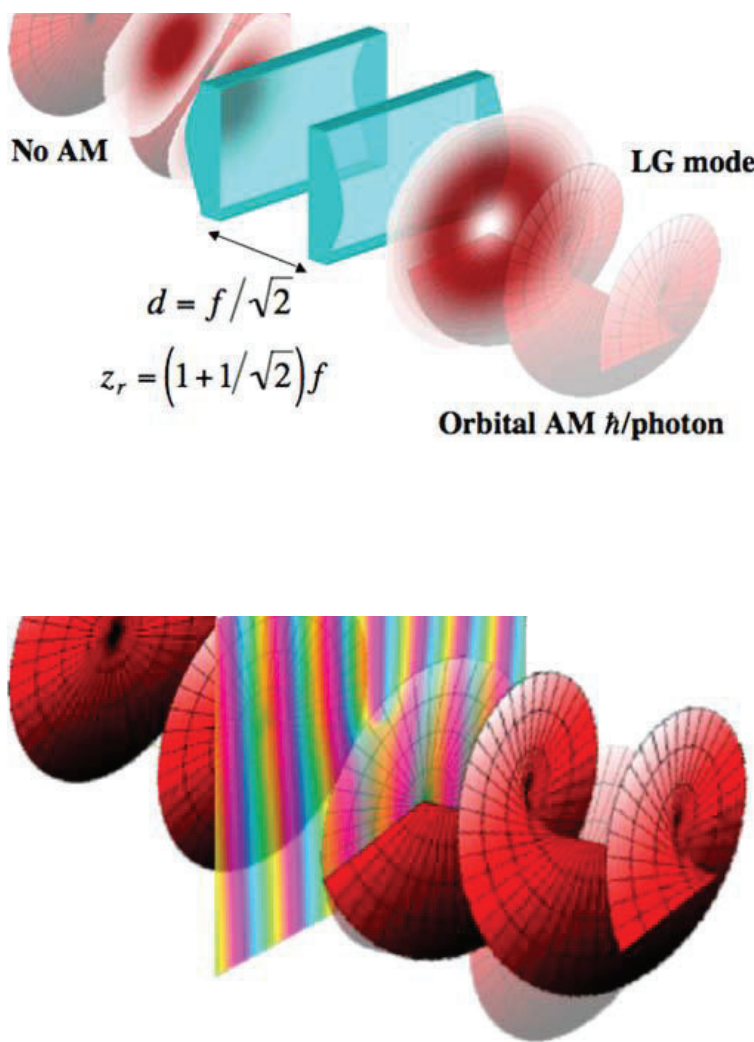

Fig. 2 Creating vortex beams with lenses (a) and holograms (b).

A number of methods have been developed for determining the topological charge. The topological charge can be determined either computationally by the use of methods like zero-crossing, line integral or phase contour methods or interferometrically where usually the vortex beam is made to interfere with its generating plane or spherical reference beam to form fork or spiral fringes as vortex signature [7]. In addition, both astigmatic and holographic optical elements can be used in the same manner as for creation, but in reverse. These methods are mainly based on measuring the intensity distribution of a vortex beam passing through various diffractive elements, or the intensity distribution resulting from the interference of a vortex beam with a reference plane wave.

Another possible way to detect an optical vortices is with use of a high sensitivity ShackHartmann wavefront sensor (Figure 3). A microlens array (MA) subdivides the wavefront
(W) into multiple beams that are focused in a CCD camera. Local slope of the wavefront over each microlens aperture determines the location of the spot on the CCD. Red arrows represent normals to the wavefront. The spot pattern deviation allows to determine the slope of the wavefront segment, thus making possible to reconstruct the wavefront and to detect the vortex beam.[8]

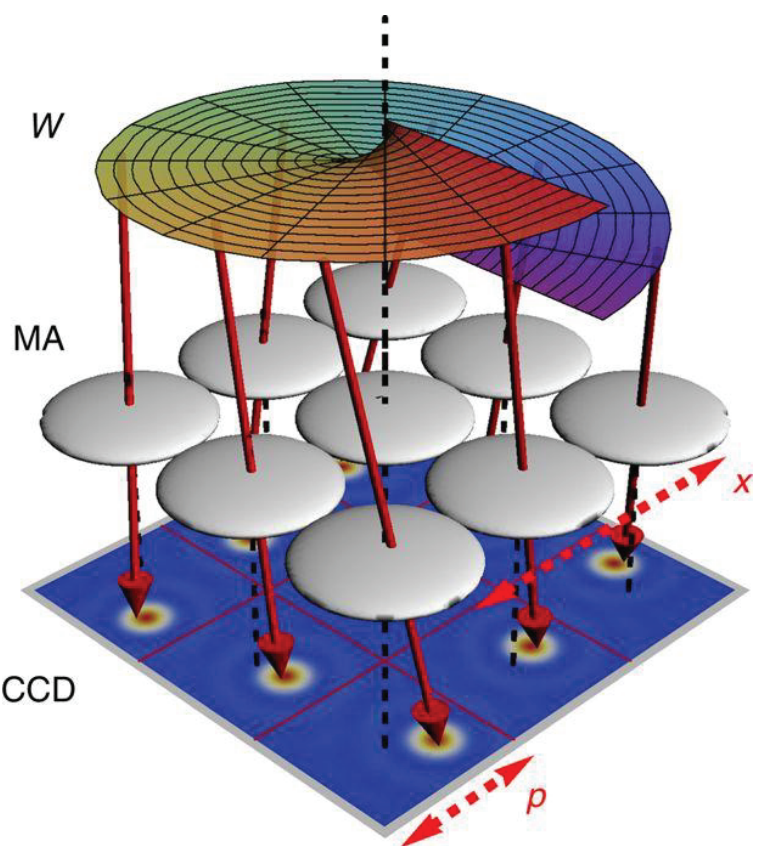

Fig. 3 Detecting vortices with S-H wavefront sensor.

However, if the light beam is polychromatic and / or partially incoherent, the topology of the phase front of the vortex beam is not clearly defined, and at the incoherent limit neither the spiral phase nor the characteristic zero intensity at the center of the vortex can be observed. To determine the topological charge of a vortex beam with a small degree of coherence, the correlation method was used in a number of papers [9]. The method is based on the fact that an annular dislocation of a low-coherent optical monochromatic vortex can be visualized using the cross-correlation function.

It should be noted that in some cases, the most complete information about the singular structure of the vortex beam is provided not by the dominant topological charge, but by the spectrum of the topological charge, which is directly related to the spectrum of the orbital angular momentum. Such cases include lowcoherent monochromatic vortices and polychromatic ones.

In this paper, shearing interferometry is employed to detect the vortex signature and 
determine its topological charge as it does not require a reference beam, it can be made as a very simple and compact optical system and its fringe pattern is less affected by air currents and vibrations [2].

In shearing interferometry, the amplitude of the input beam is divided into two, these two beams may or may not travel along the same optical path depending on the type of the interferometer. The first portion of the input beam is unaltered, while in the second half a significate parameter is changed either by shifting, rotating or reversing its wavefront with respect to the first half of the input beam.

\section{Shearing interferometry:}

Shearing interferometry basically relies on the properties of interfering light. It is the process in which an interference pattern is achieved between a test wave and its displaced replica in a region where the two waves overlap [10]. The fringe formation in the region of overlap is governed by:

For cyclic shearing interferometer

$$
O P D(x, y)=W(x, y)-W(x-\Delta x, y)
$$

For rotational shearing interferometer [11]

$$
O P D(\rho, \theta)=W(\rho, \theta-\varphi / 2)-W(\rho, \theta+
$$

For reversal shearing interferometer

$$
O P D(\rho, \theta)=W(\rho, \theta)-W\left(\rho^{\prime}, \theta^{\prime}\right)
$$

The fringe pattern optical path difference (OPD) relates to the phase gradient $\nabla \phi$ by the following relation

$$
(\nabla \Phi)_{x}=\lim _{\Delta x \rightarrow 0}(k * \Delta W / \Delta x)
$$

When the shear $\Delta \mathrm{x}$ is small, small shear approximation can be applied and one can write

$$
(\nabla \Phi)_{x}=k * \Delta W / \Delta x
$$

Where $\mathrm{k}$ is the propagation constant. If the phase deviation represented by the phase gradient $|\nabla \phi|$ is small the above relation is valid even for larger shear $\Delta \mathrm{x}[12]$.

\section{Experimental setups and results}

\subsection{Common path shearing interferometry:}

\subsubsection{Cyclic shearing interferometer}

Cyclic shearing interferometers have the advantage of low sensitivity to vibrations [2], they can be used both with incoherent and coherent light sources. Both properties are due to the fact that the optical path traveled by the two interfering beams is the same. In optical testing literature, there are already different arrangements of cyclic shearing interferometers. For this research, the triangular configuration was selected.

The interfering beams in this interferometer have near zero path difference since they counter propagate along the same path. However, there is a phase difference of $\pi$ since one beam experiences two additional total internal reflections compared to the other [2]. The Figure 4 shows the arrangement used, where an interference pattern is acquired by means of a cyclic shearing interferometer. A $5 \mathrm{~mW}$ He-Ne laser (1) with a wavelength $\lambda=$ $632.8 \mathrm{~nm}$ generates a Gaussian beam that passes through a collimator (2) to keep its beam radius from changing within enough propagation distances, the Collimated beam then illuminates the fork hologram displayed in the Holoeye transmissive LC-2002 spatial light modulator (3) generated by a computer (4) [13$14]$ and the transmitted diffracted light passes through a lens (5) with a focal length of $L_{1}=50$ $\mathrm{cm}$ exactly where the diaphragm (6) is located in order to allow only the first diffraction order carrying the vortex with the desirable topological charge to proceed through the propagation path. An identical lens (7) to the first is set $\mathrm{L}_{2}=50 \mathrm{~cm}$ apart from the diaphragm for the collimation purpose. The vortex then is split into two counter-propagating identical beams using the cyclic shearing interferometer that consists of: a beam splitter (8), mirrors ( 9 , 10) to eventually interfere with each other. The interference of the two beams is directly recorded by the CCD camera (11).

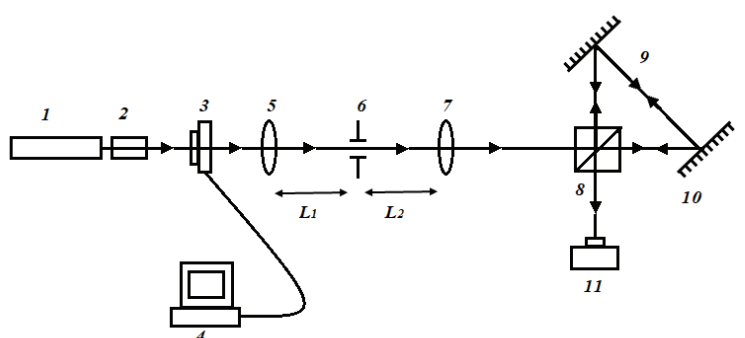

Fig. 4. The experimental set-up for interference of vortex using cyclic shearing interferometer.

Different results are obtained depending on the topological charge "m", Figure 5 shows the interference pattern for an optical vortex with topological charge $m=2$ and 4 using cyclic shearing interferometer: 


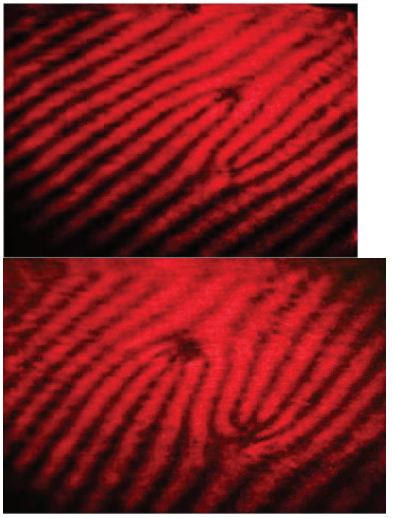

(a)

(b)

Fig. 5. Experimental interference fringes for optical vortices with different topological charges using cyclic shearing interferometer:

$$
\text { (a) } \mathrm{m}=2 \text {, (b) } \mathrm{m}=4
$$

In This figure, the interference pattern shows two oppositely oriented fork fringes due to the fact that both of the displaced wavefronts carry a vortex. The interference of each vortex infected region with the other wavefront results in the formation of a fork within the oblique linear fringes. These fringes are caused by the host Gaussian beam having a specific radius of curvature [2]. However, the presence of two oppositely oriented vortices in the shearogram corresponds to a single isolated vortex in the test wavefront. And by counting the prongs of the fork, one can easily determine the topological charge of the vortex using:

$$
m=N-1
$$

With $\mathrm{N}$ being the number of prongs.

\subsection{Non-common path shearing interferometry:}

For the following setups, the beam entering the interferometer will not cross the same path as for the cyclic interferometer since they are not common path interferometers.

\subsubsection{Rotational shearing interferometer}

In the rotational shearing interferometer, one pupil image is rotated by a small angle about the optical axis with respect to its replica (reference beam). The two images will overlap if the center of the pupil is coincided with the rotation axis where the rotation of one wavefront with respect to the other beam is denoted by $\varphi$.

The most effective and the easiest to implement rotational shearing interferometer is the variable rotational shearing interferometer [15] which is based on Michelson interferometer, where right angle prisms replace the reflecting mirrors as in Figure 6 [16].

In the following setup, the beam carrying the vortex is split into two beams by a cubic beam splitter (8), one of the beams is reflected by a fixed prism (10), while the other is reflected by a rotatable prism (9) causing the rotation of its wavefront by a certain angle with respect to their common optical axis. The resulting interferometric pattern is registered by a CCD camera.

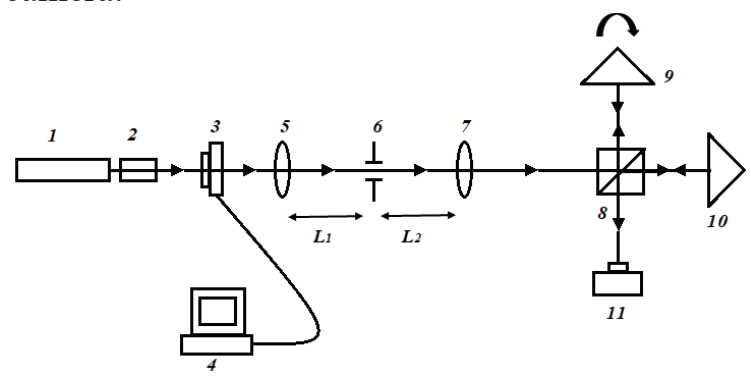

Fig. 6. Scheme of the optical vortices in rotational shearing interferometry experimental setup

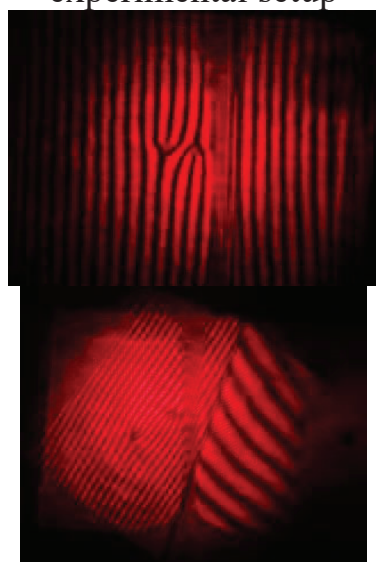

(a)

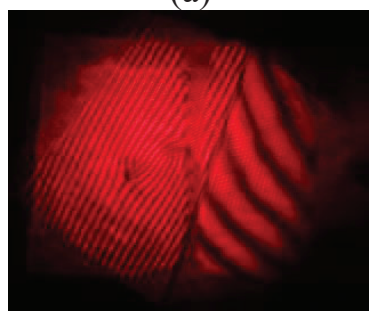

(c) (b)

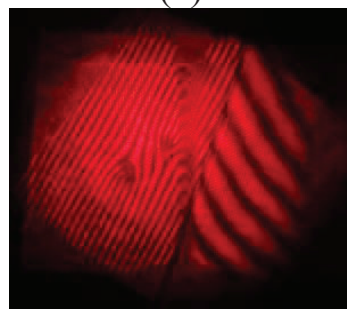

(d)
Fig. 7. Experimental interference fringes for optical vortices with different topological charges using rotational shearing interferometer when $\varphi=0^{\circ}$ (a) $\mathrm{m}=2$, and when $\varphi=30^{\circ} \mathrm{CW}$ (b) $\mathrm{m}=2$, (c) $\mathrm{m}=4$, (d) $\mathrm{m}=$ $-4$

As with the cyclic shearing interferometer, Figure 7 shows that a double forked pattern is obtained using rotational shearing 
interferometer where equation (6) is also applicable. As a result, a double fork pattern can be obtained either by using a common path or a non-common path shearing interferometers.

Another noticeable difference between the pattern of fringes for vortices with negative and positive charges using this interferometer is that the orientation of the two forks is reversed. Yet, their number of prongs is still the same. Facilitating by that the determination of the vortex charge sign and value. Also, we can see that the fork patterns of the vortex are not affected by the rotation angle. Yet, it affects the orientation of the fringes in each baseline.

In addition to this, the change of the rotation angle causes a change in the spatial frequency of the fringes in each baseline.

\subsubsection{Reversal shearing interferometer}

The reversal shearing (Fig.8) interferometer splits the input beam into two wavefronts in which one is a reversed replica of the other.

The beam will be split by the beam splitter (8) so that one of the beams is reflected by a mirror (9) while the other is horizontally flipped and reflected by the prism (10) to interfere with the first beam. The interference of the two beams is directly recorded by the CCD camera (11).

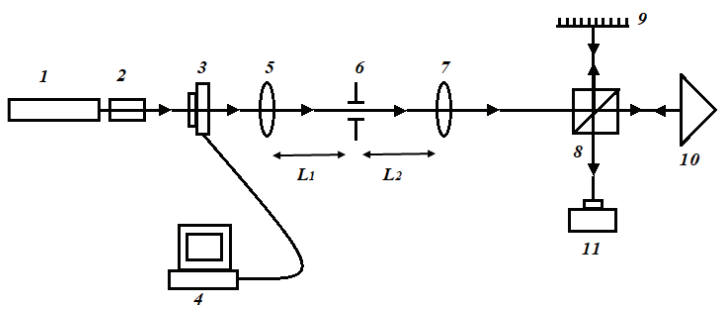

Fig. 8. Scheme of the optical vortices in reversal shearing interferometry experimental setup

Different results are obtained depending on the topological charge "m". Figure 9 shows the interference pattern for optical vortices with positive topological charge $\mathrm{m}=2,3$ using reversal shearing interferometer, while Figure 10 shows the interference pattern for optical vortices with negative topological charge $m=-$ $2,-3$, in addition to their computer simulation.

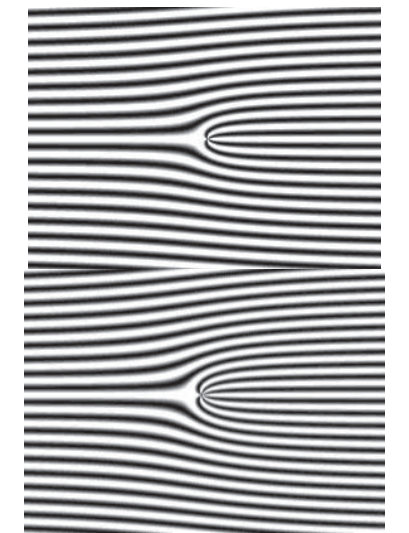

(a)

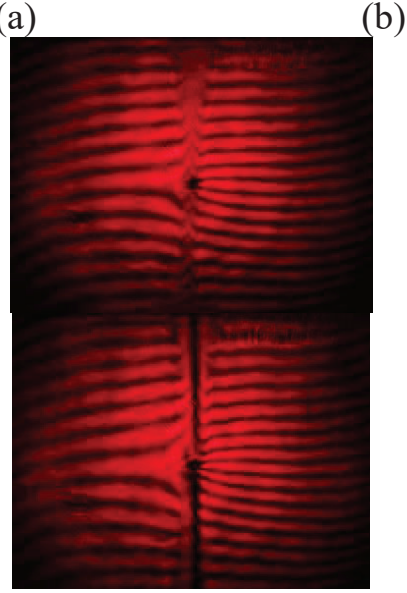

(c)

(d)

Fig. 9. Simulated (top) and experimental (bottom) interference fringes for optical vortices using reversal shearing interferometer: (a) $\mathrm{m}=2$, (b) $\mathrm{m}=3$, (c) $\mathrm{m}=2$, (d) $\mathrm{m}=3$

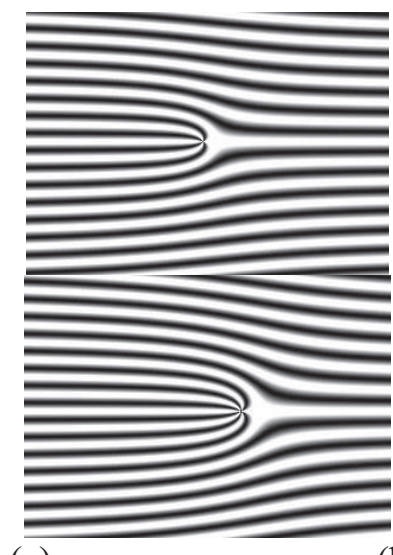

(a) (b) 


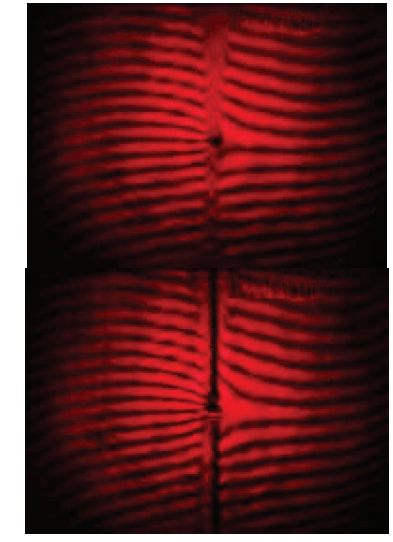

(c)

(d)

Fig. 10. Simulated (top) and experimental (bottom) interference fringes for optical vortices using reversal shearing interferometer: (a) $\mathrm{m}=-2$, (b) $\mathrm{m}=-3$, (c) $\mathrm{m}=$

$$
-2, \text { (d) } m=-3
$$

In The last two figures, we can see that the resulting interference patterns using this technique are different, they shows that with the reversal shearing interferometer, there is only one fork pattern instead of two. This fork is either facing left or right depending on the sign of the topological charge of the vortex (left for negative charges while right for positive charges).

Its corresponding simulation was achieved basically by modifying the equation of interference of a Gaussian beam with a vortex beam given as follows

$$
I=2 I_{0}[1+\cos (k x+m \theta)]
$$

With the assumption that both the Gaussian and vortex beams has equal intensities. In Cartesian coordinates, this equation will be:

$I=2 I_{0}\left[1+\cos \left(k x+m \tan ^{-1}(y / x)\right)\right]$

With $\theta \in\left[-\frac{\pi}{2}, \frac{\pi}{2}\right]$, since $\tan ^{-1}$ is an odd function, for this reason, in order to have $\theta \in$ $[0,2 \pi], \theta$ can be written as:

$$
\theta=1 / 2 * \operatorname{sgn}(x)[\operatorname{sgn}(x)-1] \pi+\tan ^{-1}(y /
$$

Resulting in:

$I=2 I_{0}[1+\cos (k x+m)$

$\left.\left.2\left(\operatorname{sgn}(x)[\operatorname{sgn}(x)-1] \pi+\tan ^{-1}(y / x)\right)\right)\right]$

(10)

On the other hand, since the linear fringes are horizontal, the reference beam can be considered as a plane wave with constant amplitude and a wave vector $\mathrm{k}$ on the $(\mathrm{y}-\mathrm{z})$ plane instead of the $(\mathrm{x}-\mathrm{z})$ plane, transforming the equation into:

$$
\begin{aligned}
& I=2 I_{0}[1+\cos (k y+m / \\
& \left.\left.2\left(\operatorname{sgn}(x)[\operatorname{sgn}(x)-1] \pi+\tan ^{-1}(y / x)\right)\right)\right]
\end{aligned}
$$

It is also seen that the spatial frequency in the direction of the fork pattern is doubled. As a result, $\mathrm{m}$ is replaced by $2 \mathrm{~m}$ in the previous equation

$I=2 I_{0}[1+\cos (k y+m(\operatorname{sgn}(x)[\operatorname{sgn}(x)-$

$\left.\left.\left.1] \pi+\tan ^{-1}(y / x)\right)\right)\right]$

(12)

Which corresponds to:

$$
I=2 I_{0}[1+\cos (k y+2 m \theta)]
$$

As a result, this expression provides a very close approximation to the results acquired experimentally and can be utilized to determine the topological charge by modifying equation (6) to:

$$
N=2|m|-1
$$

So

$$
|m|=(N-1) / 2
$$

\section{Conclusion}

In this paper, the topological charge of an optical vortex was determined using both common and non-common path shearing interferometers. As for the common path shearing interferometer, the use of cyclic shearing interferometer resulted in interference pattern similar to that of the lateral shearing interferometer [17], where two reversed oriented forks are obtained. They define the singularity infected regions within an oblique linear fringes. These linear fringes are caused by the host Gaussian beam that has a specific radius of curvature. As for the non-common path shearing Interferometers. Similarly, the use of the Rotational shearing interferometer also resulted in the formation of reversed oriented forks. Yet, the change of the rotation angle caused a change in both the spatial frequency on each baseline and the orientation of the linear fringes.

Differently, the utilization of the reversal shearing interferometer resulted in a single fork formation that has a wave vector $k$ on the $(y-z)$ plane. And the doubling of the topological charge signature, giving rise to the possibility of easily detecting the optical vortex with fractional topological charges that has:

$$
m=k / 2
$$

For any arbitrary integer number $\mathrm{k}$.

\section{Acknowledgements}


Authors are grateful to the Ministry of Science and Education of Russian Federation for funding within the Project \#8.1039.2017.

\section{References}

[1] M. J. Padgett, Orbital angular momentum 25 years on, Optics express, Vol 25, Issue 10, PP. 11265-11275, 2017.

[2] T.D. Henning, A cyclic shearing interferometer for collimating beams with short coherence, Montana state university, 1991.

[3] S. Vyas, P. Senthilkumaran, Interferometric optical vortex array generator, Applied optics, Vol. 46, No.15, 20 May 2007.

[4] L. Allen, S. M. Barnett and M. J. Padgett, Optical

Angular Momentum, Institute of Physics Publishing, Bristol, 2003.

[5] M. J. Padgett, J. Courtial, and L. Allen, Light's Orbital Angular Momentum, Physics Today, 5, PP. 35-40, 2004.

[6] David L. Andrews. Structured Light and Its Applications: An Introduction to PhaseStructured Beams and Nanoscale Optical Forces, Optics and Lasers in Engineering, Academic Press, 2008.

[7] D. P. Ghai, S. Vyas, P. Senthilkumaran, R.S. Sirohi, Detection of phase singularity using a lateral shear interferometer, Optics and Lasers in Engineering, 46, PP. 419-423, 2008.

[8] B. Stoklasa, L. Motka, J. Rehacek, Z. Hradil \& L. L. Sánchez-Soto. Wavefront sensing reveals optical coherence. Nature Communications, 5 , 3275, 2014.

[9] Pires H.D.L., Woudenberg J., van Exter M.P. Measurement of the orbital angular momentum spectrum of partially coherent beams. Optical Letters 35, 6. PP. 889-891, 2010.

[10] D. P. Ghai, S. Vyas, P. Senthilkumaran, R.S. Sirohi, Detection of phase singularity using a lateral shear interferometer, Optics and Lasers in Engineering, 46, PP. 419-423, 2008.

[11] D. Malacara, Optical Shop Testing, John Wiley and Sons, New York, 1978.

[12] D. P. Ghai, S. Vyas, P. Senthilkumaran and R. S. Sirohi, Shearograms of singular beams using wedge plate lateral shear interferometer, Optics and Lasers in Engineering, Vol 46, 797- 801, 2008.

[13] A. V. Carpentier, H. Michinel and J. R. Salgueiro, Making optical vortices with computer-generated holograms, Am. J. Phys. 76 (10), October 2008.

[14] V. P. Aksenov, V. Yu. Venediktov, A. A. Sevryugin, and I. M. Tursunov, Formation of optical vortices by the use of holograms with an Asymmetric fringe profile, Pleiades Publishing, Optics and Spectroscopy, Vol. 124, No. 2, pp. 273-277, 2018.
[15] C. Roddier, F. Roddier, J. Demarcq, Compact rotational shearing interferometer for astronomical application, Opt. Eng., Vol.28, No. 1, pp 66-70.

[16] F. Roddier, C. Roddier, J. Demarcq, $A$ rotational shearing interferometer with phasecompensated roof-prisms, J. Optics (Paris). vol. 9. No 3. 145-149, 1978.

[17] D. P. Ghai, S. Vyas, P. Senthilkumaran and R. S. Sirohi, Role of lateral shear interferometers in singular optics, Photonics2008: International conference on fiber optics and photonics, December, 2008. 


\title{
Modeling of optical frequency domain reflectometer based on self-sweeping fiber laser
}

\author{
Tkachenko A.Yu. ${ }^{a}$, Lobach I.A. ${ }^{a}$ \\ ${ }^{a}$ Institute of Automation and Electrometry SB RAS
}

\begin{abstract}
In this work, an optical frequency domain reflectometer (OFDR) based on a self-sweeping fiber laser generating sequence of individual laser modes is simulated. The effect of a small frequency change for each mode which leads to a parasitic signal in the reflectogram is taken into account. The estimations for the achievable values of the maximum fiber length and spatial resolution in the proposed OFDR scheme -10 meters and $14 \mu \mathrm{m}$, respectively - are calculated based on the modeling.
\end{abstract}

Keywords: optical frequency domain reflectometry, self-sweeping fiber laser, tunable radiation

Optical reflectometry is a key technology for monitoring optical systems as well as for distributed sensors. In the last case, the dependence of the parameters of probe radiation (intensity or optical frequency) scattered in the fiber on external physical influences (temperature or mechanical stress) is used [1]. Most of the fiber distributed sensor systems based on time domain reflectometry allows to obtain spatial resolution of the order of 1 meter. In this case, a resolution improve is limited by a significant degradation in the signal-to-noise ratio with duration decrease of probe radiation. However, sufficiently small spatial resolution is required for a number of applications. Optical frequency domain reflectometry (OFDR) is the one of approaches to reduce spatial resolution up to submillimeters scales. The principle of OFDR operation is based on the analysis of the interference between the input and scattered from the fiber signals during frequency scanning of the tunable probe radiation [2]. The interference signal obtained during frequency scanning contains information on the exact position and magnitude of the reflecting events along the fiber length through the fast Fourier transform (FFT). At the same time, the OFDR systems demonstrate high sensitivity to the reflected or scattered signal $\sim$ $100 \mathrm{~dB}$.

The key element of OFDR is tunable laser, which is rather expensive and complex device. In the work, an application a new type of tunable laser - a self-sweeping fiber laser - as a tunable source for OFDR scheme is proposed. Optical frequency is tuned due to internal processes in active medium without using external tunable elements and drivers in this laser [3]. In this case, the laser generates a regular sequence of pulses, which consist of a single longitudinal mode of the cavity. The laser frequency stepwise changes from one pulse to another by a value $d v \sim 5 \mathrm{MHz}$. The range of wavelength tuning can reach more than $\Delta \lambda \sim 23 \mathrm{~nm}(\Delta v \sim 7 \mathrm{THz})$ in the spectral region of $1 \mu \mathrm{m}$ [4]. There are other advantages of using such a source for OFDR tasks besides the scheme simplicity. In particular, strict frequency discreteness of the mode during sweeping process (one pulse corresponds to one longitudinal cavity mode) allows to obtain strictly linear frequency tuning which is required for the FFT in OFDR. The spatial resolution for OFDR can be estimated taking into account the typical characteristics of the selfsweeping fiber laser:

$$
\delta l=\frac{c}{2 n} \frac{1}{\Delta v} \sim 14 \mu \mathrm{m},
$$

where $\Delta v \sim 7 \mathrm{THz}$ is frequency sweeping range [4]. The maximum fiber length is determined by the minimum frequency sampling, which is equal to the frequency jump between pulses $d v \sim 5 \mathrm{MHz}$ and is estimated to be about:

$$
l=\frac{c}{2 n} \frac{1}{d v} \sim 20 \text { meters. }
$$

However, there are also some limiting factors. In particular, the optical frequency of each pulse has a small chirp (i.e. is varied in time), due to the nature of the sweeping effect [3]. This effect can lead to degradation of the useful signal in the OFDR system. The simulation of the signals in OFDR with Mach-Zehnder interferometer (MZI) [5] is performed taking into account the real laser parameters to evaluate the possibility of using a selfsweeping fiber laser. For this purpose, MZI interference signal with different time delay $d t$, corresponding to position of reflection in fiber is analyzed. The time 
dependence of the center frequency within each pulse (chirp) having the form

$$
\delta v=\operatorname{Atanh}\left(t / \tau_{0}\right)
$$

is taking into account in the simulation. Where $A$ - chirp amplitude (A 3-4 for ytterbium fiber laser) and $\tau_{0}$ determines the pulse duration in the approximation of the pulse shape $\operatorname{sech}^{2}\left(t / \tau_{0}\right)$ [3]. The center frequency of each pulse varied linearly from one pulse to another, which corresponds to the tuning of the sweeping laser. Then the FFT for the frequency dependence of the amplitude in interference signal is performed. In this case, the peaks of the Fourier spectrum correspond to time delay $d t$. The results for the time delay $d t=0.2 \mu$ s are presented in Fig.1. In particular, one maximum corresponding to the time delay (black line in Fig. 1) is observed in the absence of a frequency chirp $(A=0)$. However, the chirp addition leads to the appearance of multiple high-order harmonics (the red line in Fig. 1). This can lead to false detections of reflections in the fiber sensor and reduce the sensitivity of the sensor system.

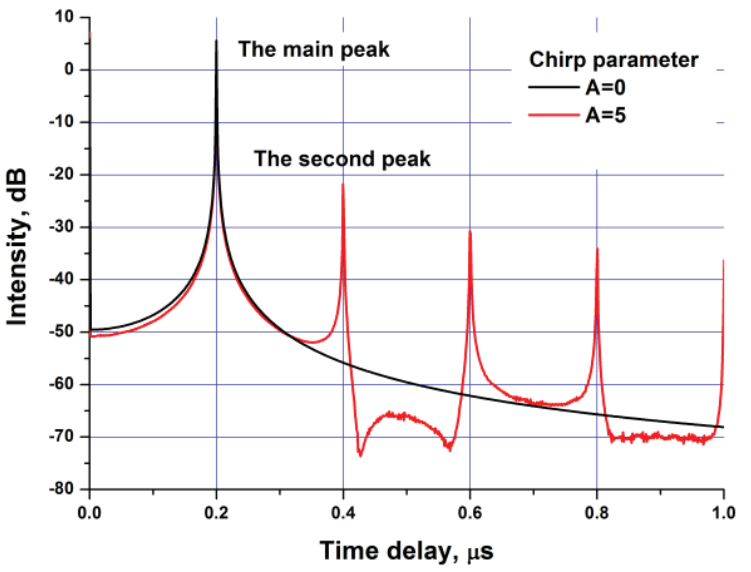

Figure.1. The Fourier spectra obtained in the OFDR scheme for input pulses with (black) and without (red) frequency chirp.

The simulation for different values of the chirp amplitude $A$ and time delay $d t$ is performed (Fig. 2). The results show the chirp effect (i.e. an increase of the amplitude for parasitic peaks) is more pronounced for longer time delays. The effect is insignificant for time delays less than $d t=0.1 \mu \mathrm{s}$ for real value of the chirp amplitude $(A \sim 3)$. This corresponds to the maximum length of the sensor of the order of 10 meters, which is less than the estimated value based on the laser frequency discreteness.

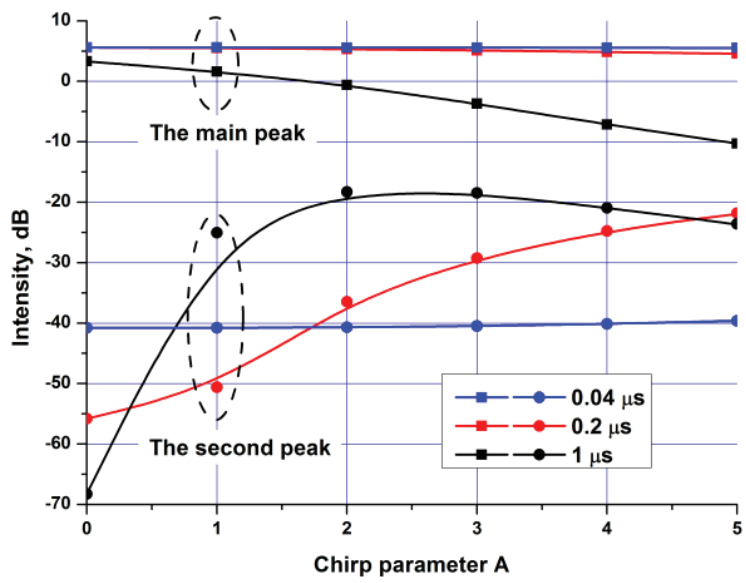

Fig.2. The amplitude of the main (squares) and the second (circles) peaks as a function of chirp amplitude at different time delays.

A more detailed description of the simulation and analysis of the results will be presented in the report. In addition, the possible approaches to increase the maximum fiber length will be proposed.

The study is funded by RFBR according to the research project № 18-32-00563.

\section{References}

[1] Arthur H. Hartog, "An Introduction to Distributed Optical Fibre Sensors", (CRC Press, 2018)

[2] MacDonald R.I., "Frequency domain optical reflectometer," Appl. Opt. 20, 1840-1844 (1981)

[3] Lobach I.A., Kablukov.S.I., Podivilov E.V., Babin S.A., "Self-scanned single-frequency operation of a fiber laser driven by a self-induced phase grating," Laser Physics Letters, 11, 045103 (2014)

[4] Lobach I.A., Tkachenko A.Yu., Kablukov S.I., "Optimization and control of sweeping range in $\mathrm{Yb}$-doped self-sweeping fiber laser," Laser Physics Letters, 13, 045104 (2016)

[5] Brian J. Soller, Dawn K. Gifford, Matthew S. Wolfe, and Mark E. Froggatt, "High resolution optical frequency domain reflectometry for characterization of components and assemblies," Opt. Express 13, 666-674 (2005) 


\section{SPIE. DIGITAL}

CONFERENCE PROCEEDINGS

$\begin{array}{lll}\text { PAPERS PRESENTATIONS JOURNALS EBOOKS } & \end{array}$

\section{Search the world's largest collection of optics and photonics applied research}

\section{SPIE. PHOTONICS GO> SPIE. MEDICAL SPIE. ADVANCED}

Newly Published Proceedings Proceedings of SPIE

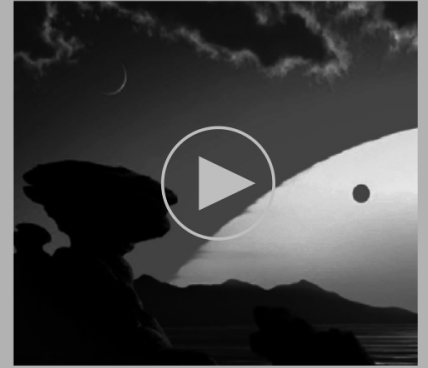

Featured Presentation

Watch the video

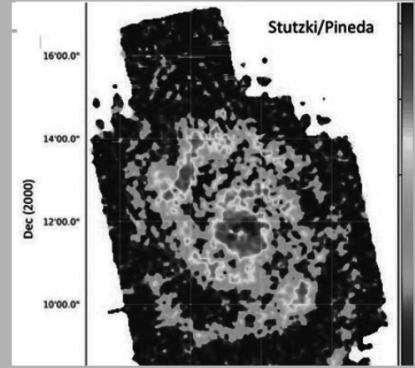

Featured Journal Article

Featuring 490,000 papers from SPIE Conferences, Journals and eBooks.

\section{wWw.spiedl.org}

